\title{
Information structures in non-cooperative games
}

Citation for published version (APA):

Perea y Monsuwe, A. (1997). Information structures in non-cooperative games. [Doctoral Thesis, Maastricht University]. Unigraphic. https://doi.org/10.26481/dis.19970626ap

Document status and date:

Published: 01/01/1997

DOI:

10.26481/dis.19970626ap

Document Version:

Publisher's PDF, also known as Version of record

\section{Please check the document version of this publication:}

- A submitted manuscript is the version of the article upon submission and before peer-review. There can be important differences between the submitted version and the official published version of record.

People interested in the research are advised to contact the author for the final version of the publication, or visit the DOI to the publisher's website.

- The final author version and the galley proof are versions of the publication after peer review.

- The final published version features the final layout of the paper including the volume, issue and page numbers.

Link to publication

\footnotetext{
General rights rights.

- You may freely distribute the URL identifying the publication in the public portal. please follow below link for the End User Agreement:

www.umlib.nl/taverne-license

Take down policy

If you believe that this document breaches copyright please contact us at:

repository@maastrichtuniversity.nl

providing details and we will investigate your claim.
}

Copyright and moral rights for the publications made accessible in the public portal are retained by the authors and/or other copyright owners and it is a condition of accessing publications that users recognise and abide by the legal requirements associated with these

- Users may download and print one copy of any publication from the public portal for the purpose of private study or research.

- You may not further distribute the material or use it for any profit-making activity or commercial gain

If the publication is distributed under the terms of Article $25 \mathrm{fa}$ of the Dutch Copyright Act, indicated by the "Taverne" license above, 
Information structures in non-cooperative games

\section{PROEFSCHRIFT}

ter verkrijging van de graad van doctor aan de Universiteit Maastricht, op gezag van Rector Magnificus, Prof. Mr. M.J. Cohen, volgens het besluit van het College van Decanen, in het openbaar te verdedigen op donderdag 26 juni 1997 om 16.00 uur.

door

Andres Perea y Monsuwé 


\section{Promotor:}

Prof. dr. H.J.M. Peters

\section{Co-promotor:}

Dr. M.J.M. Jansen

\section{Beoordelingscommissie:}

Prof. dr. F.Ch. Palm (voorzitter)

Prof. dr. E.E.C. van Damme (Katholieke Universiteit Brabant)

Prof. dr. E. Kalai (Northwestern University, USA)

Prof. dr. S.H. Tijs (Katholieke Universiteit Brabant)

Prof. dr. ir. drs. O.J. Vrieze

CIP-DATA KONINKLIJKE BIBLIOTHEEK, DEN HAAG

Perea y Monsuwé, Andres

Information structures in non-cooperative games Andres Perea y Monsuwé

Proefschrift Universiteit Maastricht

Uitgever: Unigraphic, Maastricht.

ISBN 90-5681-017-0 


\section{How it started...}

The history of this thesis starts in the fall of 1990 . At that time, I was a third year student in mathematics at the University of Aachen (Germany). Starting from the third year, you are free to choose the lectures you like (with certain restrictions, of course). One day before the beginning of the new semester, I still had to choose a third lecture. After deleting some strictly dominated lectures, I ended up with two candidates: Game Theory and some psychedelic lecture called Non-linear Operators in Infinite Dimensional Hilbert Spaces. To be honest: I didn't have the slightest idea what Game Theory was about, but for some obscure reason, I felt attracted to the name. Since I hadn't made up my mind at bedtime, I decided to toss a coin. Without realizing it, I was using a mixed strategy. The coin showed 'tails', meaning that I had to choose Non-linear Operators in Infinite Dimensional Hilbert Spaces. That night, I dreamt about being attacked by ugly operators, looking very non-linear, flying around in infinite dimensional Hilbert spaces. At three o'clock in the morning, I woke up bathing in cold sweat. Seven hours later, I found myself sitting in a small lecture hall, waiting for the Game Theory lecture to begin.

According to the Lecture Book, the lecture would be given by Hans Peters and Koos Vrieze: two names which sounded very Dutch to me. Hans Peters arrived five minutes late, a thing which is generally viewed as a huge crime in Germany. The excuse "Es gab einen Stau auf der Autobahn" didn't help too much. Before starting the lecture, Hans looked at the students and probably thought by himself: "You don't have the slightest idea what Game Theory is about".

Exactly three years later, I was a first year $\mathrm{PhD}$-student in Maastricht and my supervisor, Hans Peters, sent me to Aachen for my first teaching job. It was my task to discuss the exercises belonging to the Game Theory lecture. When I entered the lecture hall, I saw some familiar faces which I remembered from my student period. A very strange feeling indeed.

Now, in the spring of 1997 , I only have a slight idea what Game Theory is about. However, this slight idea is the basis for the thesis you have in front of you. Of course, this thesis is not only my fault. I take advantage of the situation to thank some people who where really important during the construction of this book.

First of all, I thank Hans Peters and Mathijs Jansen for being such wonderful? supervisors. They were always there to help me and I think I have been really lucky to have them as my advisors. Two very different characters, but together 
an extremely interesting combination.

Bettiria Klaus has been my room mate for more than three years and I must admit that it has never been boring with her. A good atmosphere at the office is the beginning of everything. However, 1 think that her uncountably many phone calls with Ruud could be reduced to countably many.

With Janos Flesch I played many, many snooker matches, usually decided by the last black ball. Moreover, I had the pleasure to write an article together with him. But, more importantly, thanks for being such a good friend.

I am still amazed by the mathematical skills of Dries Vermeulen, who is one of the happy few to understand Mertens stable sets and, moreover, be able to explain it to me. It was nice to have you as a co-author.

During the 1995 Game Theory conference in Jerusalem, Ehud Kalai invited me to come to Nonthwestern University (Evanston, USA), where I was a guest from april until july 1996. I still have splendid memories of this period. It was not only a very productive time, but $I$ also learned a lot from my discussions with Ehud Kalai, Jeroen Swinkels, Roger Myerson, Alejandro Manelli and others. A special thanks goes to Ehud Kalai for inviting me and Jeroen Swinkels, who took the risk to write an article together with me.

At the 1996 Game Theory conference in Bangalore (India), I met Salvador Barberà who asked me to visit Universitat Autonoma in Barcelona. In october of the same year, I spent three weeks there. It didn't take long to fall in love with the beautiful city. At the department, $\mathbb{I}$ was surrounded by a very nice group of people. I express my gratitude to Salvador Barberà for giving me the opportunity to discover Barcelona and his university.

The current and former secretaries of our department, Ellen, Karin, Miranda and Yolanda, deserve a special word of thanks for the good work they have done and for being so nice.

I am grateful to the Netherlands Organization of Scientific Research (NWO) because their financial support made my trip to Northwestern University possible. My participation at the Game Theory conference in Bangalore was sponsored by Shell Nederland N.V.

Last but not least, this thesis would never have been possible without the daily visit of Ton Storcken, speaking his legendary words "Geef toch op!". Thanks Ton. 
Een goed gebit alsmede een sterke maag

Wens ik u tae, heus waar!

Wanneer gij maas eenmaal mijn boek verdraagt, verdragen gij en ik elkaar!

Friedrich Nietzsche 


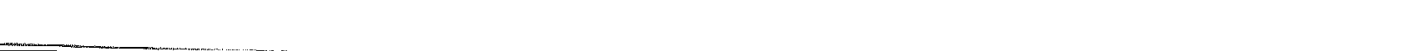




\section{Contents}

1 Introduction $\quad 5$

I Consistent assessments $\quad \mathbf{1 5}$

2 Consistency in extensive form games 17

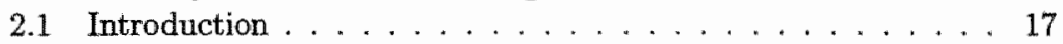

2.2 Model and definitions . . . . . . . . . . . . . . . . . 18

2.3 The main result . . . . . . . . . . . . . . . 21

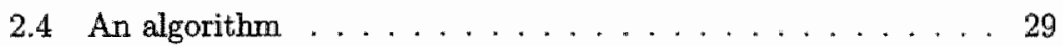

2.5 Structure of consistent assessments ........... 33

2.6 Bayesian consistency and consistency .......... 35

3 Consistency in infinite signaling games 39

3.1 Introduction . . . . . . . . . . . . . . . 39

3.2 Model and definitions . . . . . . . . . . . . . . . . . 42

3.3 Requirements for consistency . . . . . . . . . . . . 44

3.4 Strong consistency ..................... 52

3.5 Characterization of strong consistency ......... 54

3.6 Other consistency concepts . . . . . . . . . . . . . 59

3.7 Structurally consistent assessments ........... 61

II Buying information 63

4 Buying information 65

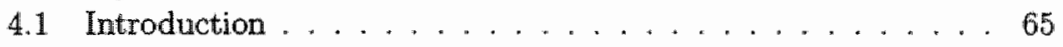

4.2 Implementation of buying information . . . . . . . . 69

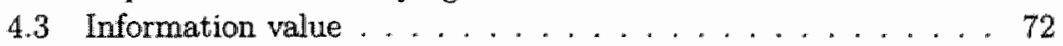

4.4 Computation of information value . . . . . . . . . . . 74

4.5 Properties of information value ............ 77 
4.6 Examples . . . . . . . . . . . . . . . . . 79

4.7 Public information transaction . . . . . . . . . . . 85

4.8 Non-single appearance games ... . . . . . . . . 89

5 Seller's commitment degree 93

5.1 Introduction . . . . . . . . . . . . . 93

5.2 Information value . . . . . . . . . . . . . . . . . 98

5.3 Case of no commitment .................. 101

5.3 .1 Extended game, strategies and beliefs ......... 101

5.3 .2 Information value . . . . . . . . . . . 103

5.4 Case of full commitment . . . . . . . . . . . . 106

5.4.1 The standard negotiation phase ........... . 107

5.4 .2 Characterization of information value ........ 107

5.5 Standard case .................................. 110

5.6 Comparison of information values ............ 111

6 Repeated games 115

6.1 Introduction . . . . . . . . . . . . . . 115

6.2 Standard model . . . . . . . . . . . . . . 117

6.2 .1 Repeated games with non-observable actions . . . . 117

6.2 .2 Characterization of lower equilibrium payoffs . . . . 119

6.3 Many information mechanisms . . . . . . . . . . . . 120

6.4 Simple lower equilibria . . . . . . . . . . . . . . . . . . 121

6.4.1 Characterization of simple lower equilibrium payoffs . . 121

6.4.2 Detectability of deviations to other information mech-

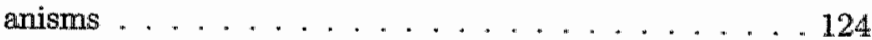

6.4 .3 Proof of characterizations ............. . . 126

\section{Other topics}

7 Player splitting 135

7.1 Introduction . . . . . . . . . . . . . . 135

7.2 Model and definitions . . . . . . . . . . . . . . . . . . . . . . . . . . . 138

7.3 Technical properties. . . . . . . . . . . . . . . . . . 140

7.4 Independent player splittings . . . . . . . . . . . . . . 142

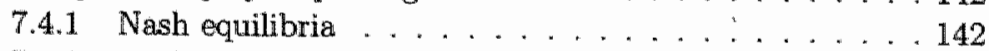

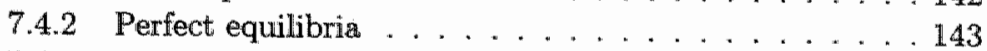

7.4 .3 Proper equilibria . . . . . . . . . . . . . . . . . . . . . . . . . . . . . . . . . . . . . . . . . . . .

7.4 .4 Stable sets . . . . . . . . . . . . . . . . . . . 148

7.5 Invariance under player splittings . . . . . . . . . . 150 
7.6 Dependent player splittings . . . . . . . . . . . 153

7.7 Single appearance structures . . . . . . . . 156

8 Limit consistent solutions $\quad 159$

8.1 Introduction . . . . . . . . . . . . . . . . . . 159

8.2 Strongly consistent solutions . . . . . . . . . . 161

8.3 Properties . . . . . . . . . . . . . . . . . . . 162

8.4 Limit consistent solutions . . . . . . . . . . . . 166

8.5 Perfect and proper equilibria . . . . . . . . . . . 168

8.6 Extensive form games . . . . . . . . . . . . . . . . . . 169

8.7 Weakly perfect equilibria . . . . . . . . . . . . . . 170

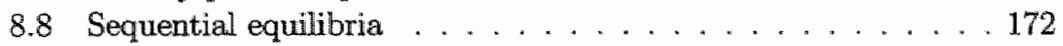

9 Costly coordination $\quad 177$

9.1 Introduction . . . . . . . . . . . . . . 177

9.2 Games with costly coordination . . . . . . . . . . . 180

9.3 Negative excess solutions . . . . . . . . . . . . . . 181

9.4 Implementation of coordination . . . . . . . . . . 184

9.5 Characterization of NE-equilibria . . . . . . . . . . . 186

9.6 Cooperative excess game . . . . . . . . . . . . . . . . . 190

9.7 Minimal excess solutions . . . . . . . . . . . . . . . . 194

9.8 Examples of minimal excess solutions . . . . . . . . 196

9.9 Characterization of ME-equilibria . . . . . . . . . . . . 200

$\begin{array}{ll}\text { Index } & 204\end{array}$

$\begin{array}{ll}\text { Bibiliography } & 207\end{array}$

Summary in Dutch 213

$\begin{array}{ll}\text { Curriculum vitae } & 219\end{array}$ 
$+$ 


\section{Chapter 1}

\section{Introduction}

Non-cooperative games are a very useful tool to model the strategic interaction between decision makers who try to maximize their individual profits but can not make binding agreements about the decisions to be taken. Think, for instance, of firms competing in a market or the relationship between an employer and an employee. In game theoretic terms, the decision makers are called players.

The timing of the decision making can be described efficiently by a so-called game tree, which consists of nodes and edges. We assume that the game tree starts at a root, which marks the beginning of the game. At the root, either a player has to make a decision or a chance move occurs. In the former case, the edges leaving the root represent the possible decisions (or actions) which can be chosen by the player whereas in the latter case they reflect the different chance moves that can occur. By a chance move we mean an event for which the outcome is completely determined by nature. The players have therefore no influence on this outcome. One could think, for example, of the chance move determining the weather for tomorrow.

Each of the edges leads to a new node, which represents a new situation in the game. This node can either be decision node, meaning that a player has to choose an action, a chance node or a terminal node. In the case of a terminal node, the game ends and each of the players receives a payoff. If it is a decision node or chance node, the edges leaving this node will lead to new nodes and so on.

In many practical situations, the players are not completely informed about the decisions which have been taken by other players or the outcomes of the chance moves. This lack of information can be modeled by so-called information sets. Formally, an information set is a collection of nodes controlled by the same player: if the play of the game reaches a node of the information set, the corresponding player only knows that one of the nodes in the infor- 
mation set is reached, without knowing which one. Therefore, information sets can be seen as a tool to model the information structure in the game. Since this thesis is concerned with information structures, a centrol role will be played by information sets.

A combination of a game tree, informations sets and payoffs at the terminal nodes is called an extensive form game. An example of an extensive form game is given in the figure below.

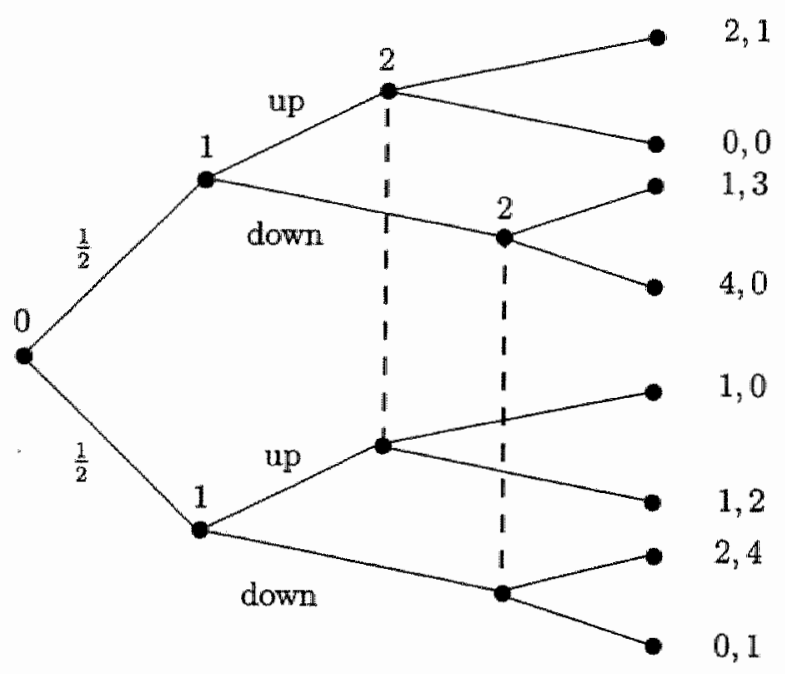

Figure 1.1

Here, the information sets are represented by dashed lines. This game starts with a chance move, denoted by the number zero, for which the outcome is observed by player 1. At each of his (singleton) information sets, player 1 can choose between two actions; up and down. Player 2 does not observe the outcome of the chance move but he sees which action has been chosen by player 1. Finally, player 2 must choose between two actions at each of his information sets.

This type of game is called a signaling game. Player 1, who observes the chance move, is called the informed player whereas player 2 is the uninformed player. The actions of player 1 are called signals (or messages) since player 2 can use them to extract information about the outcome of the chance move. 
For instance, if player 1 announces to go up at his upper node and to go down at his lower node, then player l's action will give player 2 exact information about the chance move.

A behavior strategy for player 1 in this game is to randomize betwreen 'going up' and 'going down' at both information sets. A similar definition holds for player 2. In general, a behavior strategy for a player is a complete plan which prescribes at each of his information sets a randomization over the available actions. Player 1 could, for example, decide to play ' $\frac{1}{3}$ up $+\frac{2}{3}$ down' at his upper information set and ' $\frac{1}{2}$ up $+\frac{1}{2}$ down' at his lower information set. Here, ' $\frac{1}{3}$ up $+\frac{2}{3}$ down' means that with probability $\frac{1}{3}$ he will go up and with probability $\frac{2}{3}$ he will go down.

We assume that the players hold correct conjectures about the behavior strategies played by the opponents. If player 2 knows that player 1 plays the behavior strategy described above, and he observes that player 1 has gone up, then he can conclude that the upper chance move has occurred with probability $\frac{2}{5}$ and the lower chance move has occurred with probability $\frac{3}{5}$. We say that player 2 has beliefs $\left(\frac{2}{5}, \frac{3}{5}\right)$ at his upper information set.

Formally, a belief system for a player prescribes at each of his information sets a probability distribution on the nodes in this information set. Such a probability distribution can be interpreted as the player's conjecture about the probabilities that each of the nodes in this information set is reached. In the example, player 2 could derive the beliefs at his upper information set using his knowledge about player l's behavior strategy and the probabilities of the chance move. In the same way, he can derive the beliefs at his lower information set. In technical terms, we say that player 2 derives his beliefs using Bayesian updating. The combination of behavior strategies and beliefs, which is obtained in this way, is called an assessment. Since the beliefs are obtained by Bayesian updating, we call the assessment Bayesian consistent.

However, suppose that player 1 decides to go down with probability one at both information sets. If player 2 arrives at his upper information set, he can not use Bayesian updating since he knows that this information set would not be reached if player 1 would have played his announced behavior strategy. Nevertheless, player 2 has to form beliefs at this information set. At first sight, it might seem reasonable to allow for all possible beliefs at information sets which are reached with probability zero. But, as is shown in the example below, this can lead to implausible beliefs. 


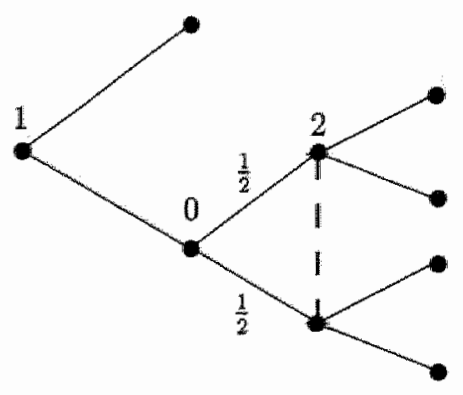

Figure 1.2

Assume that player 1 goes up with probability one, meaning that player 2's information set is reached with probability zero. By requiring only Bayesian consistency, player 2 would be free to choose his beliefs as he likes. However, the only plausible beliefs are $\left(\frac{1}{2}, \frac{1}{2}\right)$ since player 2 knows the probabilities of the chance move. A possible way to overcome this problem is by considering small perturbations of the announced behavior strategies. Suppose that, with some very small probability, player 1 makes a mistake and goes down. In this case, the only beliefs that can be obtained by Bayesian updating are the beliefs $\left(\frac{1}{2}, \frac{1}{2}\right)$, no matter how small the mistake probability. Hence, by letting the mistake probability tend to zero, we end up with a unique belief system, namely the beliefs $\left(\frac{1}{2}, \frac{1}{2}\right)$. The assessments obtained in this way are called consistent.

This concept can be generalized for arbitrary extensive form garnes: an assessment is called consistent if it is the limit of a sequence of Bayesian consistent assessments in which all actions are played with some positive minimal probability. It can be seen easily that every consistent assessment is automaticaily Bayesian consistent. Part I of this thesis is devoted to the analysis of the consistency concept.

Since the definition of consistency makes use of sequences and limits, it is in general very hard to check whether a given assessment is consistent or not. In Chapter 2, however, we present a purely algebraic characterization of consistent assessments in extensive form games, which makes no use of sequences and limits. This characterization makes it easier to verify if an assessment is consistent or not. Moreover, the characterization is used to develop an algorithm which computes the set of all consistent assessments 
for a given extensive form game. Finally, we exploit the characterization to analyze the geometric structure of the set of consistent assessments.

Let us return to the signaling game of Figure 1.1. It turns out that consistency in this game is equivalent to Bayesian consistency, meaning that every Bayesian consistent assessment is also consistent. This is a result which can be shown for general finite signaling games. By finite we mean that there are only a finite number of chance moves and players 1 and 2 have a finite number of actions at each of their information sets.

Now, suppose that we allow for signaling games with (possibly uncountably) infinitely many chance moves and actions. In this case, there exists a generally accepted definition for Bayesian consistency but it is not clear how consistency should be defined for infinite signaling games. This problem is due to the fact that convergence of assessments causes many problems in this case. In Chapter 3, we investigate several ways to develop a 'good' definition for consistency in infinite signaling games. First of all, we present a definition of consistency which we call strang consistency and show that every appropriate consistency concept is a refinement of strong consistency. In this chapter, we describe formally what we mean by an 'appropriate' consistency concept. Moreover, we give an easy to verify characterization of strong consistency.

One could say that, starting from a behavior strategy profile, consistency selects those belief systems that are, in some particular sense, rational given these behavior strategies. However, it also seems interesting to look at the other direction. Starting from a belief system, which behavior strategies would be rational given these beliefs?

A possible way to answer this question is by requiring sequential rationality. An assessment is said to be sequentially rational if at each information set, the corresponding player maximizes his expected payoff, given his beliefs at this information set and given the behavior strategies at other information sets. In the signaling game of figure 1.1, going up at the upper information set would be sequentially rational for player 2 if his beliefs at this information set are $\left(\frac{3}{4}, \frac{1}{4}\right)$ whereas going down at this information set is sequentially rational if his beliefs would be $\left(\frac{1}{2}, \frac{1}{2}\right)$.

By combining the two conditions mentioned above, consistency and sequential rationality, we arrive at the so-called sequential equilibrim concept (Kreps and Wilson, 1982). An assessment is said to be a sequential equilibrium if it is both consistent and sequentially rational. Hence, the sequential equilibrium concept selects those assessments where the beliefs are chosen in a rational way, given the strategies, and the strategies are chosen rationally, given the beliefs. It is this mutual dependence between beliefs and strategies 
that plays a central role in the concept.

Now, suppose that there would be an information seller in the signaling game who could sell player 2 information about the chance move. If player 2 would buy this information at his upper information set, the seller will tell him which of the two nodes in this information set has been reached. Techwically speaking, the upper information set would be split into two singleton information sets.

If player 2's beliefs at his upper information set would be $\left(\frac{1}{2}, \frac{1}{2}\right)$, he could guarantee the expected payoff 1 at this information set without buying information. On the other hand, player 2 could obtain expected payoff $\frac{3}{2}$ by buying the information, since he could decide to go up if he knows that the upper node has been reached whereas he can go down after observing that the game has reached the lower node of the information set. Therefore, player 2 is prepared to pay at most $\frac{1}{2}$ for information at his upper information set. This concept can be generalized by assuming that a monopolistic information seller sells information to the players of an extensive form game (from now on called information buyers). Formally, information is modeled by partitions that divide information sets into disjoint subsets of nodes. If a buyer buys a partition at one of his information sets, the seller will tell him which subset of the partition has been reached by the play of the game. It is this situation that we want to focus on in Part II of the thesis.

In Chapters 4 and 5, we ask ourselves the question: How much money can the seller possibly extract from the buyers, given that both seller and buyers act in a rational way? The question is answered by looking at a new extensive form game, called the extended game, which is obtained in the following way. The extended game is played by the seller and the buyers. The seller's task is to choose prices for all pertitions that are offered to the buyers. At every information set, the corresponding buyer can buy one of the available partitions and subsequently choose an action contingent on the subset of the partition that has been reached. At the end of the game, the seller's payoff is his expected income from selling partitions and the payoff for a buyer is simply the difference between his original payoff and the amount that he has paid for buying information. The requirement that both seller and buyers should act rationally is modeled by assuming that seller and buyers play a sequential equilibrium in this extended game. Therefore, the maximum amount of money that the seller can expect within this context is the highest possible payoff for the seller in a sequential equilibrium of the extended game. This maximum payoff for the seller is called the information value.

However, the information value depends crucially on the way in which the seller chooses the prices for the partitions. In Chapter 4, we assume that 
the seller chooses all the prices before the actual game starts. The menu of prices is observed by all buyers but the the partition bought at a certain information set is not publicly observed. In this situation, we provide an efficient method to compute the information value. Moreover, it is shown that making information transactions public can either increase or decrease the information value.

In Chapter 5, we analyze how the information value changes if we change the way in which the seller chooses the prices. Three different cases are considered. In the first case, which is called the no commitment case, the information seller must choose the price of a partition at the moment that the corresponding information set (i.e. the information set at which the partition is offered) is reached. As a consequence, sequential rationality for the seller implies that the seller can not commit to choose prices at an information set which would not be optimal at the moment that this information set is reached. Therefore, one could say that the seller has no commitment power in this case.

In the second case, which is called the full commitment case, the seller can include an arbitrary negotiation phase between himself and the buyers before the actual game starts. In this negotiation phase, the seller can make binding agreements about prices that he will charge for partitions and, moreover, he can charge the buyers certain amounts for offering a specific menu of prices. Hence, the seller posesses maximal commitment power in this situation.

The last case is the situation analyzed in Chapter 4 and is therefore called the standard case. In this situation, the seller has partial commitment power since he is able to charge suboptimal prices at information sets in order to raise his income. However, he does not have full commitment power. Therefore, with respect to the seller's commitment degree, the standard case lies in between the no commitment case and the full commitment case.

We show that the information value of the full commitment case is always at least as high as the information value in the standard case, which, in turn, cannot be less than in the no commitment case. This result supports the intuition that increasing the seller's commitment power leads to a higher income for the seller.

Often, decision makers face the same strategic situation many times. For example, firms competing on a market must choose a price for the product at the beginning of every period. Such situations can be modeled efficiently by repeated games. In a repeated game, the players play the same non-cooperative game infinitely many times. In many interesting cases, the players are not fully aware of the actions that have been chosen by the other players in previous periods. Formally, this can be modeled by so-called infor- 
mation mechanisms which assigns to every profile of actions a private signal for a player.

In Chapter 6 , we present a model in which the players of such a repeated game can buy an information mechanism before playing the game. It is assumed that the prices for the information mechanisms are fixed. Within this context, we characterize the set of payoffs which can be obtained by so-called lower equitibria of the resulting game. Without going into technical details, we just mention that lower equilibria are a particular version of Nash echulibria for the repeated game with buying information. Important is that the characterization is given completely in terms of the one-shot game (i.e. the game which is repeated infinitely many times).

In Part III, we deal with other topics in non-cooperative game theory. Chapter 7 analyzes the situation in which the players of an extensive form game decide to decentralize their decisions. Formally, this is modeled by assuming that the players distribute their information sets among so-called agents. In the signaling game of Figure 1.1, for instance, player 1 could decide to assign his two information sets to agents $1 \mathrm{a}$ and $1 \mathrm{~b}$, whereas player 2 can distribute his information sets among agents $2 \mathrm{a}$ and $2 \mathrm{~b}$. In this way, we obtain a game with four players in which the agents $1 \mathrm{a}$ and $1 \mathrm{~b}$ receive the original player 1's payoffs and the agents $2 \mathrm{a}$ and $2 \mathrm{~b}$ obtain the payoffs of player 2 . Such an operation, in which information sets are distributed among agents, is called a player splitting. We investigate how different solutions concepts, such as Nash equilibria, (normal form) perfect equilibria, (normal form) proper equilibria and stable sets, behave under player splittings.

It turns out that the player splitting constructed above does not change the Nash equilibria, perfect equilibria and stable sets of the signaling game. This depends crucially on the fact that on every path in the game tree, at most one agent of player 1 and player 2 is present. We say that the player splitting is independent.

Generally, a player splitting is called independent if every path in the game tree contains at most one agent of every player. Intuitively, this means that the decisions are decentralized in such a way that it is impossible that two different agents of the same have player have to reach a decision during a play of the game. We show that the Nash equilibria, perfect equilibria and stable sets of an extensive form game do not change under independent player splittings. By giving a counterexample, we illustrate the fact that the set of proper equilibria can change under independent player splittings.

Afterwards, we go one step further by proving that the class of independent player splittings is the largest class of player splittings under which Nash equilibria, perfect equilibria and stable sets do not change. This result yields 
a characterization of the class of independent player splittings.

In Chapter 8 , we focus on consistency of non-cooperative solution concepts. This notion of consistency is not related to the consistency concept analyzed in Part I. In this definition of consistency, the notion of reduced games plays a central role. Starting from a game and a strategy profile for the players, we can reduce the game by selecting a coalition of players and assuming that the players outside the coalition are kept fixed at their respective strategies in the profile. In this way we obtain a new game which is only played by the players in this coalition. Roughly speaking, a solution is called strongly consistent if applying the solution to a reduced game is the same as taking the solution of the original game and projecting it onto the reduced game. It is well known that there is no non-empty, strongly consistent Nash equilibrium refinement other than the Nash equilibrium concept itself. However, we show that refinements such as perfect equilibria and proper equilibria in normal form games and weakly perfect equilibria and sequential equilibria in extensive form games can be approximated by a sequence of strongly consistent solutions. We say that these solutions are limit consistent.

Finally, in Chapter 9, we investigate non-cooperative games in which the players can coordinate their actions against certain costs. However, players are not able to make binding agreements about the strategies that will be played. In the solutions we present, so-called excesses of coalitions play a central role. The excess of a coalition at a certain strategy profile is defined as the highest aggregate payoff which can be obtained by this coalition if the players in the coalition coordinate their actions and deviate collectively to some other strategy profile.

The first solution concept which is considered is the negative excess solution which selects those strategy profiles at which the excesses of all coalitions are non-positive. These are exactly the strategy profiles where no coalition has an incentive to deviate. However, in many cases the negative excess solution turns out to be empty.

This is a reason to consider a second solution concept, which selects those strategy profiles where the vector of excesses is minimal with respect to some given relation on the space of excess vectors. This concept is called the minimal excess solution and has the advantage that it is always nonempty. Both the negative excess solution and the minimal excess solution are characterized by the so-called local deviation game, an extensive form game which is introduced formally in Chapter 9. 


\section{Part I}

\section{Consistent assessments}





\section{Chapter 2}

\section{Characterization of consistent assessments in extensive form games}

\subsection{Introduction}

This chapter is based on Perea y Monsuwe et al. (1996b).

In an extensive form game, a combination of a behavior strategy profile and a belief system is called an assessment. Such an assessment is a sequential equilibrium (Kreps and Wilson, 1982) if it satisfies sequential rationality and consistency. A sequential equilibrium can be viewed as properly extending Selten's ideas of (subgame) perfectness $(1965,1975)$. The first condition, sequential rationality, is equivalent to a system of polynomial inequalities and is therefore straightforward to check. Checking consistency, however, is in general much harder because the definition requires sequences of assessments. The central result of this chapter (Theorem 2.3.1) is a purely algebraic characterization of consistent assessments: It does not make any use of sequences and limits, but characterizes consistent assessments by two conditions. The first condition is an algebraic restriction pertaining to the supports of the strategies and beliefs. It turns out that checking this restriction is equivar lent to solving a linear program: an insight which plays an important role in the algorithm indicated below. The second condition of the characterization implies that we can put mistake probabilities on the actions played with probability zero in such a way that the relative beliefs are equal to the relative realization probabilities of the corresponding nodes. The relationship between relative probabilities and consistency of assessments is also considered in Kohlberg and Reny (1991) and McLennan (1989a,b). 
This central result will be applied in several ways.

First, its proof implies that a consistent assessment can always be approximated by a sequence of completely mixed assessments of a very simple form, determined by just a few parameters.

Second, based on this characterization an algorithm is developed to compute the set of consistent assessments in a given extensive form game.

Third, the characterization makes it possible to give a geometrical description of the set of consistent assessments. As a byproduct of this description, it can be shown rather easily that the set of consistent assessments is semi-algebraic, which means that it can be described by a finite number of polynomial inequalities. Since sequential rationality is equivalent to a finite system of polynomial inequalities, it follows directly that the set of sequential equilibria is semi-algebraic; a result which has already been shown by Blume and Zame (1994).

Fourth, we obtain a (purely algebraic) characterization of those extensive form games in which every Bayesian consistent assessment is consistent. (Bayesian consistency is the weaker requirement that beliefs should satisfy Bayes' rule.) As is well known, signalling games are examples of such games. The organization of the chapter is as follows. In section 2 basic concepts and notations are introduced. Section 3 contains the central result, i.e., the algebrailc characterization of consistent assessments. It also presents the first consequence of this result about simple sequences of assessments as mentioned above, as a corollary. Moreover, an example is provided to illustrate how the central result can be used to check whether a given assessment is consistent. In section 4, we develop an algorithm which explicitly cornputes the set of consistent assessments in an extensive form game. Also, an example is provided. In section 5, we describe the geometrical structure of the set of consistent assessments. Finally, section 6 provides a characterization of the class of extensive form games for which Bayesian consistency and consistency coincide.

Further discussion of related literature is given at the relevant places throughout the chapter.

\subsection{Model and definitions}

\section{Notations}

$\mathbb{R}^{*}:=\mathbb{R} \cup\{-\infty\}$. For every $x \in \mathbb{R}$ we define $x+(-\infty):=-\infty$. Moreover, $(-\infty)+(-\infty):=-\infty$ whereas $(-\infty)-(-\infty)$ is not defined.

For a finite set $A, \Delta(A)$ is the set of all probability distributions on $A$. 
For a matrix $M$ we denote the transposed matrix by $M^{t}$.

\section{Extensive form games}

An extensive form structure is a quintet $\mathcal{S}=\langle K, P, H, A, \tau\rangle$, where

(1) $K$ is a rooted tree with root $x_{0}$. The set of terminal nodes is denoted by $Z$ and the set of non-terminal nodes except $x_{0}$ is denoted by $X$. The unique sequence of nodes and edges connecting the root $x_{0}$ and a terminal node $z$ is called the poth from $x_{0}$ to $z$. For an $x \in X \cup\left\{x_{0}\right\}, E(x)$ is the set of edges leaving the node $x$.

(2) $P: X \rightarrow\{0,1,2, \ldots, n\}$ and $P(x)$ is the player that controls the node $x$. If $P(x)=0$ then the node $x$ represents a situation where a chance move occurs. Such nodes will be called chance nodes.

(3) $H$ is an $n$-tuple $\left(H_{1}, H_{2}, \ldots, H_{n}\right)$, where $H_{i}$ is a partition of the set $P^{-1}(i)$ into information sets (or agents) such that for every information set $h$ the following conditions are satisfied:

(a) every path from $x_{0}$ to (an element of) $Z$ intersects $h$ at most once

(b) $|E(x)|=|E(y)|$ for all $x, y \in h$.

We also use the letter $H$ for the set $U_{i} H_{i}$ of all information sets.

(4) for every information set $h, A(h)$ is a partition of the set of edges $\bigcup_{x \in h} E(x)$ leaving the information set $h$ into actions such that $|E(x) \cap a|=1$ for any action $a \in A(h)$ and $x \in h$. We will assume that $|A(h)| \geq 2$ for all $h \in H$.

(5) $\tau$ is a function which defines at every chance node a strictly positive probability distribution on the set of edges which leave the chance node. These edges represent the different chance moves.

An extensive form game is a pair $\langle\mathcal{S}, u\rangle$, where $S$ is an extensive form structure and $u$ is an $n$-tuple $\left(u_{1}, u_{2}, \ldots, u_{n}\right)$, where $u_{i}: Z \rightarrow \mathbb{R}$. The function $u_{i}$ is the payoff function for player $i$.

We assume that the extensive form games considered have perfect recall (see Kuhn, 1953), which means that two paths leading to the same player $i$ information set contain the same player $i$ actions. 


\section{Consistent assessments and sequential equilibria}

A behavior strategy profile (BSP) is a function $\sigma$ which assigns to every information set $h$ a probability distribution $\sigma_{h}$ on $A(h)$. A belief system is a function $\beta$ which assigns to every information set $h$ a probability distribution $\beta_{h}$ on the nodes in this information set. A combination $(\sigma, \beta)$ of a BSP and a belief system is called an assessment.

An assessment $(\sigma, \beta)$ is called Bayesian consistent if at every information set which is reached with positive probability the beliefs are derived according to Bayes' rule. So for every $h$ with $\mathbb{P}_{\sigma}(h)>0$ it must hold that

$$
\beta_{h}(x)=\frac{\mathbb{P}_{\sigma}(x)}{\mathbb{P}_{\sigma}(h)}
$$

for every $x \in h$. Here, $\mathbb{P}_{\sigma}(x)$ and $\mathbb{P}_{\sigma}(h)$ denote the probabilities that the node $x$ and the information set $h$ are reached, respectively, if $\sigma$ is played.

An assessment $(\sigma, \beta)$ is called consistent if there is a sequence $\left(\sigma^{k}, \beta^{k}\right)_{k \in \mathbb{N}}$ of completely mixed, Bayesian consistent assessments converging to $(\sigma, \beta)$. Completely mixed means that every action is played with positive probability. Obviously, consistency implies Bayesian consistency.

An assessment $(\sigma, \beta)$ is called sequentially rational if at every information set, the corresponding player maximizes his expected payoff given his beliefs at this information set and given the local strategies at the other information sets. Mathematically, this means that for every player $i$ and $h \in H_{i}$

$$
\sum_{x \in h} \beta_{h}(x) U_{i}(\sigma \mid x) \geq \sum_{x \in h} \beta_{h}(x) U_{i}(\sigma \backslash a \mid x)
$$

for all $a \in A(h)$. Here, $U_{i}(\sigma \mid x)$ denotes the expected payoff for player $i$ if the game would start in the node $x$ and the players would play according to $\sigma$. By $\sigma \backslash a$ we denote the BSP in which the action $a$ is played at information set $h$ and at all other information sets, the players act according to $\sigma$. The expression $U_{i}(\sigma \backslash a \mid x)$ is defined analogously to $U_{i}(\sigma \mid x)$.

This definition of sequential rationality, which is taken from Myerson (1991), can be regarded as a "local" definition since it only takes into account deviations at single information sets. However, the definition of sequential rationality by Kreps and Wilson (1982) allows a player to deviate at all his information sets and can therefore be seen as a 'global definition'. Despite the fact that global sequential rationality is strictly stronger than local sequential rationality, both definitions turn out to be equivalent if the assessment is consistent, as is shown in Hendon et al. (1996). Consequently, either defi- 
nition of sequential rationality can be used for defining sequential equilibria. 1

An assessment $(\sigma, \beta)$ is called a sequential equilibrium if it is consistent and sequentially rational.

\subsection{The main result}

Before formulating our main result, we need some further definitions.

For an assessment $(\sigma, \beta), A^{+}(\sigma)$ denotes the set of actions played with positive probability and by $X^{+}(\beta)$ we mean the set of nodes with positive belief. The restriction of $\sigma$ on the actions in $A^{+}(\sigma)$ is denoted by $\sigma^{+}$. By $A^{0}(\sigma)$ we denote the set of actions played with probability zero whereas $X^{0}(\beta)$ denotes the set of nodes with belief zero.

A pseudo-BSP is a system $\bar{\sigma}=\left(\bar{\sigma}_{h}\right)_{h \in H}$ of functions $\bar{\sigma}_{h}: A(h) \rightarrow[0,1]$. In contrast with BSP's, the sum of the probabilities of the actions in $A(h)$ does not need to be equal to 1 in a psendo-BSP. A pseudo-BSP $\bar{\sigma}$ is called completely mixed if every action is played with positive probability.

For a pseudo-BSP $\bar{\sigma}, \mathbb{P}_{\bar{\sigma}}(x)$ and $\mathbb{P}_{\bar{\sigma}}(h)$ are defined in a similar way as for a BSP.

The following theorem gives an algebraic characterization of consistent assessments. In the theorem, we denote by $A_{x}$ the set of actions which occur on the path to the node $x$.

Theorem 2.3.1 Let $\Gamma$ be an extensive form game and $(\sigma, \beta)$ an assessment. Then, $(\sigma, \beta)$ is consistent if and only if

(1) there are numbers $\{\varepsilon(\alpha)\}_{a \in A^{0}(\sigma)} \in(0,1)$ such that

$$
\prod_{a \in A^{0}(\sigma) \cap A_{x}} \varepsilon(a)=\prod_{a \in A^{0}(\sigma) \cap A_{y}} \varepsilon(a)
$$

for all nodes $x_{n} y \in X^{+}(\beta)$ in the same information set and

$$
\prod_{a \in A^{0}(\sigma) \cap A_{x}} \varepsilon(a)<\prod_{a \in A^{0}(\sigma) \cap A_{y}} \varepsilon(a)
$$

for all nodes $x, y$ in the same information set with $x \in X^{0}(\beta)$ and $y \in X^{+}(\beta)$ and

\footnotetext{
${ }^{9}$ For Bayesian consistent assessments the two definitions of sequential rationality are dif ferent. In some situations, the set of 'global' sequential best responses to a given Bayesian consistent assessment may be empty.
} 
(2) $\sigma^{+}$can be extended to a completely mixed pseudo-BSP $\bar{a}$ such that

$$
\frac{\mathbb{P}_{\tilde{\sigma}}(x)}{\mathbb{P}_{\tilde{\sigma}}(y)}=\frac{\beta(x)}{\beta(y)}
$$

for all nodes $x, y \in X^{+}(\beta)$ in the same information set.

Intuitively, condition (1) says that we can put mistake probabilities on the zero probability actions such that $\beta$ places positive belief exactly on those nodes which are reached with maximum mistake probability. Therefore, this condition checks whether the combination $\left(A^{+}(\sigma), X^{+}(\beta)\right)$ is possible in a consistent assessment. Condition (2) states that we can put mistake probabilities on the zero probability actions such that the relative probabilities of the nodes with positive belief are equal to the relative beliefs.

Condition (1) is somewhat related to the notion of a b-labelling, used by Kreps and Wilson (1982) in Lemma A1. Furthermore, there is a connection between condition (2) and the mapping $\left(\mu^{b}, \pi^{b}\right)$ which can be found in Lemma A2 of the same paper.

Before proving this theorem, we give an example in order to illustrate the meaning of conditions (1) and (2) in the theorem. Moreover, this example shows how the characterization can be used to check whether a given assessment is fully consistent or not.

Example 2.1. Consider an extensive form game with the following extensive form structure. 


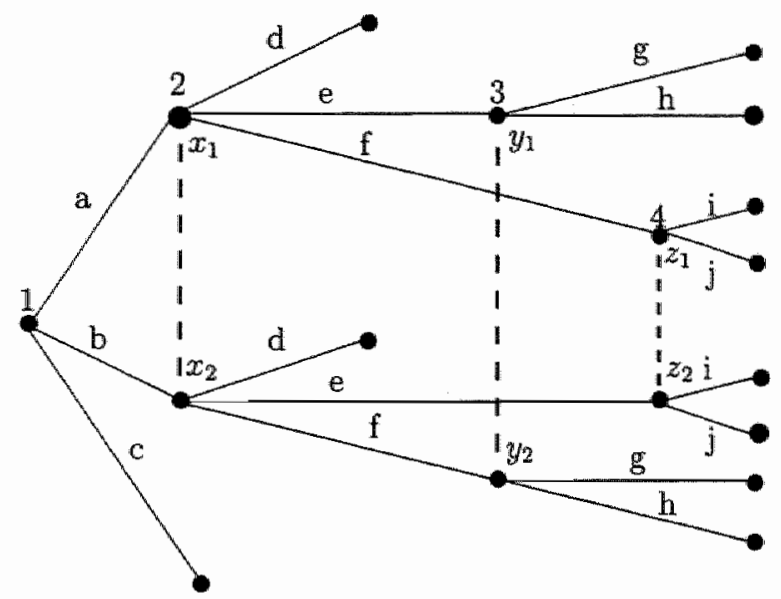

Figure 2.1

This extensive form structure is also used by Kohlberg and Reny (1991) in Figure 5.

Consider an assessment $(\sigma, \beta)$ with $\sigma(c)=\sigma(d)=1, \beta\left(x_{1}\right)=\frac{1}{3}, \beta\left(y_{1}\right)=$ $\frac{1}{5}$ and $\beta\left(z_{1}\right)=\frac{1}{2}$. We apply Theorem 2.3 .1 in order to verify if $(\sigma, \beta)$ is consistent.

We can extend $\sigma^{+}$to a completely mixed pseudo-BSP $\bar{\sigma}$ by defining

$$
\bar{\sigma}(a)=\frac{1}{3}, \bar{\sigma}(b)=\frac{2}{3}, \bar{\sigma}(e)=\frac{3}{10} \text { and } \bar{\sigma}(f)=\frac{6}{10} .
$$

Obviously, it holds that

$$
\frac{\mathbb{P}_{\sigma}(x)}{\mathbb{P}_{\tilde{\sigma}}(y)}=\frac{\beta(x)}{\beta(y)}
$$

for all $x, y$ lying in the same information set. Hence, condition (2) in Theorem 2.3 .1 is satisfied.

Condition (1) in this theorem is also satisfied by choosing

$$
\varepsilon(a)=\varepsilon(b)=\varepsilon(e)=\varepsilon(f)=\frac{1}{2} .
$$

Therefore, we may conclude that $(\sigma, \beta)$ is consistent.

Now, consider an assessment $(\sigma, \beta)$ with $\sigma(c)=\sigma(d)=1, \beta\left(x_{1}\right)=0, \beta\left(y_{1}\right)=$ $\frac{1}{3}$ and $\beta\left(z_{1}\right)=\frac{2}{3}$.

Assume, that condition (1) in Theorem 2.3.1 would be satisfied for some 
$\varepsilon(a), \varepsilon(b), \varepsilon(c)$ and $\varepsilon(d)$. Since $\beta\left(x_{1}\right)=0$ and $\beta\left(x_{2}\right)>0$ it follows that $\varepsilon(a)<\varepsilon(b)$. Furthermore, it must hold that $\varepsilon(a) \cdot \varepsilon(e)=\varepsilon(b) \cdot \varepsilon(f)$ which implies that $\varepsilon(e)>\varepsilon(f)$. However, this would mean that $\varepsilon(a) \cdot \varepsilon(f)<\varepsilon(b) \cdot \varepsilon(e)$ which is a contradiction since $z_{1}, z_{2} \in X^{+}(\beta)$. Hence, condition (1) can not be satisfied. By Theorem 2.3.1, $(\sigma, \beta)$ is not a consistent assessment.

Proof of Theorem 2.3.1. Let $(\sigma, \beta)$ be an assessment. For convenience, we write $A^{+}, A^{0}, X^{+}$and $X^{0}$ instead of $A^{+}(\sigma), A^{0}(\sigma), X^{+}(\beta)$ and $X^{0}(\beta)$. For every information set $h$, let $r(h)$ be the first node in $h$ with positive belief. (We assume, for convenience, that the nodes in an information set are ordered.) For a node $x \in h$, let $r(x):=r(h)$. We construct the matrix $M$ as follows. The rows of the matrix correspond with the nodes in $X$ and the columns with the actions in $A^{0}$, so $M=\left(m_{x, a}\right)_{x \in X, a \in A^{0}}$. The elements $m_{x, a}$ are given by

$$
m_{x, a}:=\left\{\begin{aligned}
1 & \text { if } a \in A_{x} \text { and } a \notin A_{r(x)} \\
-1 & \text { if } a \notin A_{x} \text { and } a \in A_{r(x)} \\
0 & \text { otherwise. }
\end{aligned}\right.
$$

Furthermore, we define the vector $s=\left(s_{x}\right)_{x \in X}$ by

$$
s_{x}:=\sum_{c \in C_{x}} \log \tau(c)+\sum_{a \in A^{+} \cap A_{x}} \log \sigma(a)-\sum_{c \in C_{r(x)}} \log \tau(c)-\sum_{a \in A^{+} \cap A_{r(x)}} \log \sigma(a) .
$$

Here, $C_{x}$ is the collection of chance moves on the path to $x$ and $\tau(c)$ is the positive probability that the chance move $c$ occurs.

Let the vector $b=\left(b_{x}\right)_{x \in X}$ be given by

$$
b_{x}:=\log \beta(x)-\log \beta(r(x)),
$$

where $\log 0:=-\infty$. Note that $b_{x}$ can be $-\infty$ since $\beta(x)$ can be 0 .

Lemma 2.3.2 The assessment $(\sigma, \beta)$ is consistent if and only if there is a sequence $\left(w^{k}\right)_{k \in \mathbb{N}}$ in $\mathbb{R}^{A^{0}}$ converging coordinatewise to $-\infty$ such that $b=$ $s+\lim _{k \rightarrow \infty} M w^{k}$.

Proof. " $\Rightarrow$ " Suppose that $(\sigma, \beta)$ is a consistent assessment with supporting sequence $\left(\sigma^{k}, \beta^{k}\right)_{k \in \mathbb{N}}$. For every $h \in H$ and $x \in h$ we have

$$
\frac{\beta(x)}{\beta(r(x))}=\lim _{k \rightarrow \infty} \frac{\mathbb{P}_{\sigma^{k}}(x)}{\mathbb{P}_{\sigma^{k}}(r(x))}
$$

for every $x \in X$. By definition,

$$
\mathbb{P}_{\sigma^{k}}(x)=\prod_{c \in C_{x}} \tau(c) \cdot \prod_{a \in A_{x} \cap A^{+}} \sigma^{k}(a) \cdot \prod_{a \in A_{x} \cap A^{0}} \sigma^{k}(a)
$$


for every $x$. Since $\lim _{k \rightarrow \infty} \sigma^{k}(a)=\sigma(a)>0$ for every $a \in A^{+}$, it follows that

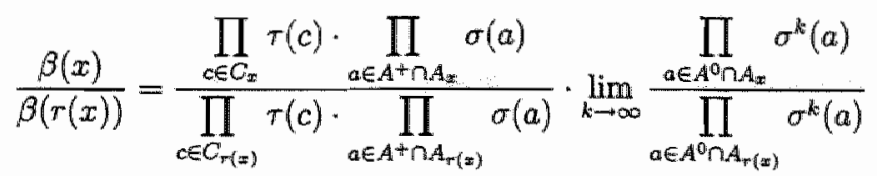

for every $x$. If we take the logarithm on both sides, we obtain

$$
b_{x}=s_{x}+\lim _{k \rightarrow \infty} \sum_{a \in A^{0}} m_{x, a} \log \sigma^{k}(a) .
$$

If we define the sequence $w^{k}$ by $w_{a}^{k}:=\log \sigma^{k}(a)$ for every $a \in A^{0}$, it follows that $w^{k}$ converges coordinatewise to $-\infty$ and $b=s+\lim _{k \rightarrow \infty} M w^{k}$.

" $\Leftarrow$ " Let the node $x$ be fixed and let $w^{k}$ be a sequence as described in Lemma 2.3.2. Then,

$$
b_{x}=s_{x}+\lim _{k \rightarrow \infty} \sum_{a \in A^{0}} m_{x, a} w_{a^{*}}^{k}
$$

By definition of the elements $m_{x, a}$ it holds that

$$
b_{x}=s_{x}+\lim _{k \rightarrow \infty}\left[\sum_{a \in A^{0} \cap A_{x}} w_{a}^{k}-\sum_{a \in A^{0} \cap A_{\mathrm{r}(x)}} w_{a}^{k}\right] .
$$

If we take the exponential function on both sides, we obtain

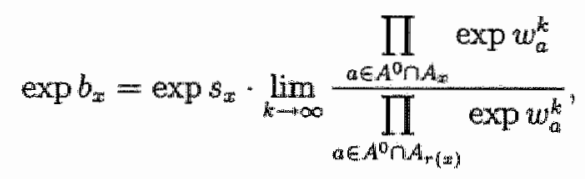

where $\exp (-\infty):=0$. Using the definition of $b_{x}$ and $s_{x}$ yields

$$
\frac{\beta(x)}{\beta(r(x))}=\frac{\prod_{c \in C_{x}} \tau(c) \cdot \prod_{a \in A^{+} \cap A_{x}} \sigma(a)}{\prod_{c \in C_{r(x)}} \tau(c) \cdot \prod_{a \in A^{+} \cap A_{A_{(x)}}} \sigma(a)} \cdot \lim _{k \rightarrow \infty} \frac{\prod_{a \in A^{0} \cap A_{x}} \exp w_{a}^{k}}{\prod_{a \in A^{0} \cap A_{r(x)}} \exp w_{a}^{k}}
$$

We define the sequence $\left(\hat{\sigma}^{k}\right)_{k \in \mathbb{N}}$ by

$$
\hat{\sigma}^{k}(a):= \begin{cases}\exp w_{a}^{k} & \text { if } a \in A^{0} \\ \sigma(a) & \text { if } a \in A^{+}\end{cases}
$$


Obviously, the sequence $\left(\hat{\sigma}^{k}\right)_{k \in N}$ converges to $\sigma$. For every $k$, let the BSP $\sigma^{k}$ be given by

$$
\sigma^{k}(a):=R^{k}(a) \cdot \hat{\sigma}^{k}(a)
$$

where $R^{k}(a):=\frac{1}{\sum_{a^{*} \in A(h)} \hat{\sigma}^{k}\left(a^{r}\right)}$ and $h$ is the information set at which the action $a$ is available.

Since $R^{k}(a)$ converges to 1 for every $a$ and $\hat{\sigma}^{k}(a)>0$ for every $a$ it follows that $\left(\sigma^{k}\right)_{k \in \mathbb{N}}$ is a sequence of completely mixed BSP's converging to $\sigma$. With equation (2.1) it follows that

$$
\frac{\beta(x)}{\beta(r(x))}=\lim _{k \rightarrow \infty} \frac{\prod_{c \in C_{x}} \tau(c) \cdot \prod_{a \in A^{+} \cap A_{z}} \sigma^{k}(a)}{\prod_{c \in C_{r(x)}} \tau(c) \cdot \prod_{a \in A^{+} \cap A_{r(x)}} \sigma^{k}(a)} \cdot \frac{\prod_{a \in A^{0} \cap A_{x}} \sigma^{k}(a)}{\prod_{a \in A^{0} \cap \cap A_{r(x)}} \sigma^{k}(a)} .
$$

This equality implies that

$$
\frac{\beta(x)}{\beta(r(x))}=\lim _{k \rightarrow \infty} \frac{\mathbb{P}_{\sigma^{k}}(x)}{\mathbb{P}_{\sigma^{k}}(r(x))}
$$

for every $x \in X$ and therefore

$$
\beta(x)=\lim _{h \rightarrow \infty} \frac{\mathbb{P}_{\sigma^{k}}(x)}{\mathbb{P}_{\sigma^{k}}(h)}
$$

for every $x \in X$. Hence, $(\sigma, \beta)$ is consistent. This completes the proof of Lemma 2.3.2.

Let $M^{+}$and $M^{0}$ be the restrictions of $M$ to the rows corresponding to nodes in $X^{+}$and $X^{0}$ respectively and let $s^{+}, b^{+}$be the restrictions of the vectors $s, b$ to nodes in $X^{+}$.

Lemma 2.3.3 There is a sequence $\left(w^{k}\right)_{k \in \mathbb{N}}$ in $\mathbb{R}^{A^{0}}$ converging coordinatewise to $-\infty$ such that $b=s+\lim _{k \rightarrow \infty} M w^{k}$ if and only if

(1) there is a vector $w \in \mathbb{R}^{A^{0}}$, $w<0$ with $M^{+} w=0$ and $M^{0} w<0$ and (2) $b^{+} \in s^{+}+\operatorname{Im}\left(M^{+}\right)$.

Here, $\operatorname{Im}\left(M^{+}\right)$denotes the image of the linear operator $M^{+}$.

Proof. " $\Rightarrow "$ Assume, that $\left(w^{k}\right)_{k \in \mathbb{N}}$ is a sequence as described in Lemma 2.3.3. Let the vector $v$ be given by $v:=b-s$ and let $v^{+}$be the restriction of $v$ to the nodes in $X^{+}$. By construction, $v_{x} \in \mathbb{R}$ for all $x \in X^{+}$and $v_{x}=-\infty$ 
for all $x \notin X^{+}$. Since $\lim _{k \rightarrow \infty} M^{+} w^{k}=v^{+}$and $\operatorname{Im}\left(M^{+}\right)$is closed, it follows that $v^{+} \in \operatorname{Im}\left(M^{+}\right)$, so $b^{+} \in s^{+}+\operatorname{Im}\left(M^{+}\right)$. Now, let $z \in \mathbb{R}^{A^{0}}$ with $M^{+} z=v^{+}$.

Let $B:=\left\{w \in \mathbb{R}^{A^{0}} \mid w \leq-1, M^{0} w \leq-1\right\}$ and $C:=\left\{M^{+} w \mid w \in B\right\}$. (The inequality $w \leq-1$ should be read coordinatewise.) Obviously, $B$ is a closed and convex set. Moreover, $B$ is non-empty since $w^{k} \in B$ for large $k$. It follows that $C$ is a non-empty closed convex set. Suppose, that $0 \notin C$. By Corallary 11.4.2 in Rockafellar (1970), there exists a hyperplane which separates the sets $C$ and $\{0\}$ strongly. In other words, we can find a non-zero vector $p$ and a number $\alpha \in \mathbb{R}$ such that $p \cdot c>\alpha$ for all $c \in C$ and $p \cdot 0<\alpha$. The last inequality implies that $\alpha>0$. From the first inequality, it follows that $p \cdot M^{+} w>\alpha$ for every $w \in B$. Since $w^{k}-z \in B$ for large $k$, it follows that $\lim _{k \rightarrow \infty} p \cdot M^{+}\left(w^{k}-z\right) \geq \alpha$. We know that $\lim _{k \rightarrow \infty} M^{+} w^{k}=v^{+}=M^{+} z$. Therefore $0 \geq \alpha$, which is a contradiction. So $0 \in C$, which implies that there is a $w \in \mathbb{R}^{A^{0}}, w \leq-1$ with $M^{0} w \leq-1$ and $M^{+} w=0$.

This direction could also be shown applying the theorem on the existence of approximate solutions used in Kohlberg and Reny (1991).

$" \Leftarrow$ Let (1) and (2) be satisfied and let $z \in \mathbb{R}^{A^{0}}$ with $b^{+}=s^{+}+M^{+} z$. Define the sequence $\left(w^{k}\right)_{k \in \mathbb{N}}$ by $w^{k}:=z+k w$. It is easy to check that $\left(w^{k}\right)_{k \in \mathbb{N}}$ has the desired properties.

Combining Lemma 2.3.2 and Lemma 2.3.3 leads to the following result.

Lemma 2.3.4 The assessment $(\sigma, \beta)$ is consistent if and only if

(1) there is a vector $w<0$ with $M^{+} w=0$ and $M^{0} w<0$ and

(2) $b^{+} \in s^{+}+\operatorname{Im}\left(M^{+}\right)$.

Now, we are able to prove Theorem 2.3.1.

$" \Rightarrow$ " Let $(\sigma, \beta)$ be consistent. By (2) in Lemma 2.3.4 there is a $z \in \mathbb{R}^{A^{0}}$ such that $b^{+}=s^{+}+M^{+} z$. We define the pseudo-BSP $\tilde{\sigma}$ by

$$
\bar{\sigma}(a):= \begin{cases}\sigma(a) & \text { if } a \notin A^{0} \\ \exp \left(z_{a}\right) & \text { if } a \in A^{0}\end{cases}
$$

Since

$$
b_{x}=s_{x}+\sum_{a \in A^{0}} m_{x, a} z_{a}=s_{x}+\sum_{a \in A^{0} \cap A_{x}} z_{a}-\sum_{a \in A^{0} \cap A_{\left.A^{(x)}\right)}} z_{a}
$$

for every $x \in X^{+}$, we obtain by taking the exponential function on both sides and using the definitions of $b_{x}, v_{x}$ and $m_{x, a}$ that

$$
\frac{\beta(x)}{\beta(r(x))}=\frac{\prod_{c \in C_{x}} \tau(c) \cdot \prod_{a \in A^{+} \cap A_{x}} \sigma(a)}{\prod_{c \in C_{r(x)}} \tau(c) \cdot \prod_{a \in A^{+} \cap A_{\tau(x)}} \sigma(a)} \cdot \frac{\prod_{a \in A^{0} \cap A_{x x}} \bar{\sigma}(a)}{\prod_{a \in A^{0} \cap A_{r(x)}} \sigma(a)}
$$




$$
=\frac{\mathbb{P}_{\tilde{\sigma}}(x)}{\mathbb{P}_{\tilde{\sigma}}(r(x))}
$$

for every $x \in X^{+}$which implies condition (2) in Theorem 2.3.1.

Since $M^{+} w=0$ it follows that

$$
\sum_{a \in A^{0} \cap A_{x}} w_{a}-\sum_{\left.a \in A^{0} \cap A_{r(x)}\right)} w_{a}=0
$$

for every $x \in X^{+}$. Taking the exponential function on both sides leads to the equation

$$
\frac{\prod_{a \in A^{0} \cap A_{x}} \exp w_{a}}{\prod_{a \in A^{\infty} \cap A_{r(x)}} \exp w_{a}}=1
$$

for every $x \in X^{+}$. Since $M^{0} w<0$, we can show in a similar way that

$$
\frac{\prod_{a \in A^{0} \cap A_{x}} \exp w_{a}}{\prod_{a \in A^{0} \cap A_{r(x)}} \exp w_{a}}<1
$$

for every $x \in X^{0}$. Finally, we define the constants $\varepsilon(a)$ by $\varepsilon(a):=\exp \left(w_{a}\right)$ for every $a \in A^{0}$.

Since the proof in the other direction is similar, the proof of Theorem 2.3.1 is complete. 
Consistency and simple sequences of assessments

As a corollary of Theorem 2.3.1, we show that we can restrict ourselves to a very special class of sequences of completely mixed assessments if we want to check whether a given assessment is consistent or not. These sequences, which we call simple, have the property that they are completely determined by assigning two parameters to every action. As a consequence, the infinitely dimensional problem of checking consistency is reduced to a finitely dimensional problem.

A sequence $\left(\sigma^{k}, \beta^{k}\right)_{k \in \mathbb{N}}$ of assessments is called simple if for every action a there are numbers $\bar{\sigma}(a)>0$ and $\varepsilon(a) \in(0,1]$ such that

$$
\sigma^{k}(a)=R^{k}(h) \cdot \ddot{\sigma}(a) \cdot(\varepsilon(a))^{k}
$$

for every $k \in \mathbb{N}$. Here, $h$ is the information set with $a \in A(h)$ and $R^{k}(h)$ is the normalizing constant given by $R^{k}(h)=\left[\sum_{a^{\prime} \in A(h)} \bar{\sigma}\left(a^{\prime}\right) \cdot\left(\varepsilon\left(a^{\prime}\right)\right)^{k}\right]^{-1}$.

Corollary 2.3.5 An assessment $(\sigma, \beta)$ is consistent if and only if there is a simple sequence $\left(\sigma^{k}, \beta^{k}\right)$ of completely mixed, Bayesian consistent assessments converging to $(\sigma, \beta)$.

Proof. We only have to prove the 'only if' part, since the 'if' part is true by definition. Let $(\sigma, \beta)$ be a consistent assessment. By Theorem 2.3.1 we can find numbers $\varepsilon(a)$ and $\bar{\sigma}(a)$ for every $a \in A^{0}(\sigma)$ such that the conditions (1) and (2) in this theorem are satisfied. For every $a \in A^{+}(\sigma)$ we define $\bar{\sigma}(a):=\sigma(a)$ and $\varepsilon(a):=1$. Since the simple sequence $\left(\sigma^{k}, \beta^{k}\right)$ of completely mixed, Bayesian consistent assessments induced by these numbers converges to $(\sigma, \beta)$, the proof is complete.

The notion of simple sequences of assessments is somewhat related to the sequences used by Kreps and Wilson (1982) in the proof of Lemma A2. However, the sequences in Kreps and Wilson are constructed in a different way.

\subsection{An algorithm}

In this section we provide an algorithm to compute the set of consistent assessments in an extensive form game. First, we introduce some further notation and discuss several lemmas which play an essential rolle in the development of the algorithm. 
In an extensive form game $\Gamma$, we denote the set of consistent assessments by $A^{c}$. For given sets $A^{+}$and $X^{+}, \mathcal{A}^{c}\left(A^{+}, X^{+}\right)$denotes the set of consistent assessments $(\sigma, \beta)$ with $A^{*}(\sigma)=A^{+}$and $X^{+}(\beta)=X^{+}$. Obviously,

$$
\mathcal{A}^{c}=\bigcup_{A^{+}, X^{+}} \mathcal{A}^{c}\left(A^{+}, X^{+}\right)
$$

In the proof of Theorem 2.3.1 we constructed for a given assessment $\left(\sigma_{z} \beta\right)$ a matrix $M$. However, this matrix only depends on the sets $A^{+}(\sigma)$ and $X^{+}(\beta)$. This means that we can construct such a matrix $M$ for every possible combination $A^{+}, X^{+}$.

Furthermore, we introduced for a given assessment $(\sigma, \beta)$ the vectors $b$ and $s$, where $b$ depends on $\beta$ and $s$ depends on $\sigma$. Therefore, we denote these vectors by $b(\beta)$ and $s(\sigma)$ respectively. By $b^{+}(\beta)$ and $s^{+}(\sigma)$ we denote restrictions of $b(\beta)$ and $s(\sigma)$ to $X^{+}$.

From Lemma 2.3 .4 it follows that an assessment $(\sigma, \beta)$ cen only be consistent if there is a vector $w<0$ with $M^{+} w=0$ and $M^{0} w<0$. Obviously, this problem is equivalent to the problem "Is there a vector $w \leq-1$ with $M^{+} w=$ 0 and $M^{0} w \leq-1^{2}$ ? ". The latter problem is an LP problem and can therefore be solved efficiently (by using the simplex method, for example) Combining this insight with Lemma 2.3.4 leads to the following lemma, which turns out to be the key-stone for our algorithm.

Lemma 2.4.1 Let $A^{+}, X^{+}$be given and $M$ the corresponding matrix.

If there is a vector $w \leq-1$ with $M^{+} w=0$ and $M^{0} w \leq-1$ then $\mathcal{A}^{c}\left(A^{+}, X^{+}\right)$ is equal to the set of assessments $(\sigma, \beta)$ with $A^{+}(\sigma)=A^{+}, X^{+}(\beta)=X^{+}$and $b^{+}(\beta) \in s^{+}(\sigma)+\operatorname{Im}\left(M^{+}\right)$.

Otherwise, $\mathcal{A}^{c}\left(A^{+}, X^{+}\right)$is empty.

The proof follows directly from Lemma 2.3.4.

Lemma 2.4.2 There is a basis for $\operatorname{Ker}\left(\left(M^{+}\right)^{t}\right)$ which consists of integer vectors.

Proof. Since $\left(M^{+}\right)^{t}$ is an integer matrix, we can transform $\left(M^{+}\right)^{*}$ with the Gauss-elimination method into a rational upper-triangular matrix. Obviously, the kernel of this triangular matrix (and, hence, the kernel of $\left.\left(M^{+}\right)^{t}\right)$ has a besis consisting of rational vectors. By multiplying these vectors with an appropriate integer, we obtain a basis consisting of integer vectors.

\footnotetext{
${ }^{2}$ The inequalities $w \leq-1$ and $M^{0} w \leq-1$ should be read coordinatewise.
} 
Lemma 2.4.3 Let $n^{n}, \ldots, n^{r}$ be a basis for $\operatorname{Ker}\left(\left(M^{+}\right)^{t}\right)$ consisting of integer vectors. Then,

$$
\operatorname{Im}\left(M^{+}\right)=\left\{z \mid n^{i} \cdot z=0 \text { for } i=1, \ldots, r\right\} .
$$

The proof of this lemma is straightforward.

From Lemma 2.4.1 and Lemma 2.4.3 it follows that $b^{+}(\beta)-s^{+}(\sigma) \in \operatorname{Im}\left(M^{+}\right)$ if and only if $n^{i} \cdot b^{+}(\beta)=n^{i} \cdot s^{+}(\sigma)$ for all $i$. This result leads to the following corollary.

Corollary 2.4.4 Let $n^{1}, \ldots, n^{r}$ be a basis for $\operatorname{Ker}\left(\left(M^{+}\right)^{t}\right)$. If there is a vector $w \leq-1$ with $M^{+} w=0$ and $M^{0} w \leq-1$ then $\mathcal{A}^{\mathrm{c}}\left(A^{+}, X^{+}\right)$is equal to the set of assessments $(\sigma, \beta)$ with $A^{+}(\sigma)=A^{+}, X^{+}(\beta)=X^{+}$and

$$
n^{i} \cdot b^{+}(\beta)=n^{i} \cdot s^{+}(\sigma) \text { for all } i \text {. }
$$

Otherwise, $\mathcal{A}^{c}\left(A^{+}, X^{+}\right)$is empty.

Now, we are able to construct an algorithm which generates the set of consistent assessments. The algorithm is based on the following steps.

\section{Step 1.}

Choose $A^{+}, X^{+}$and compute the corresponding matrix $M$.

\section{Step 2 .}

Solve the LP-problem "Is there a vector $w \leq-1$ with $M^{+} w=0$ and $M^{0} w \leq-1$ ? " with the simplex method.

If the answer is "yes", then goto step 3 .

If the answer is "no", then $\mathcal{A}^{\mathrm{C}}\left(A^{+}, X^{+}\right)$is empty and goto step 1 (until every combination $A^{+}, X^{+}$has been chosen).

\section{Step 3 .}

Compute a basis $n^{1}, \ldots, n^{r}$ for $\operatorname{Ker}\left(\left(M^{+}\right)^{2}\right)$ consisting of integer vectors with the Gauss-elimination method.

Then $\mathcal{A}^{c}\left(A^{+}, X^{+}\right)$is equal to the set of assessments $(\sigma, \beta)$ with $A^{+}(\sigma)=A^{+}$, $X^{+}(\beta)=X^{+}$and $n^{i} \cdot b^{+}(\beta)=n^{i} \cdot s^{+}(\sigma)$ for all $i$.

Goto step 1 (until every combination $A^{+}, X^{+}$has been chosen).

If we translate the linear equations in step 3 into the original strategies $\sigma$ and the original beliefs $\beta$ by taking the exponential function on both sides, we obtain a system of polynomial equations in $\sigma$ and $\beta$. A different algorithm to compute such polynomial equations can be found in Kohlberg and Reny (1991). 
In the following example, we apply the algorithm in order to compute one particular set $\mathcal{A}^{c}\left(A^{+}, X^{+}\right)$for the extensive form game of Example 2.1 .

Example 2.2. Let $\Gamma$ be an extensive form game with the extensive form structure of Figure 2.1.

Step 1. Choose $A^{+}=\{c, d, g, h, i, j\}$ and $X^{+}=\left\{x_{1}, x_{2}, y_{1}, y_{2}, z_{1}, z_{2}\right\}$. Hence, $A^{0}=\{a, b, e, f\}$ and $X^{0}$ is empty. The nodes $r(x)$ are given by

$$
r\left(x_{1}\right)=r\left(x_{2}\right)=x_{1}, r\left(y_{1}\right)=r\left(y_{2}\right)=y_{1}, r\left(z_{1}\right)=r\left(z_{2}\right)=z_{1} .
$$

The corresponding matrix $M$ is given by

$$
M=\left[\begin{array}{rrrr}
a & b & e & f \\
0 & 0 & 0 & 0 \\
-1 & 1 & 0 & 0 \\
0 & 0 & 0 & 0 \\
-1 & 1 & -1 & 1 \\
0 & 0 & 0 & 0 \\
-1 & 1 & 1 & -1
\end{array}\right] \begin{aligned}
& x_{1} \\
& x_{2} \\
& y_{1} \\
& y_{2} \\
& z_{1} \\
& z_{2}
\end{aligned}
$$

Step 2. Since $X^{+}=X$, we have that $M^{+}=M$ and $M^{0}=\varnothing$. There is a vector $w \leq-1$ with $M^{+} w=0$. (Take, for example, $w=[-1,-1,-1,-1]$ )

Step 3. The transposed matrix $\left(\left(M^{+}\right)^{t}\right)$ is given by

$$
\left(M^{+}\right)^{t}=\left[\begin{array}{rrrrrr}
0 & -1 & 0 & -1 & 0 & -1 \\
0 & 1 & 0 & 1 & 0 & 1 \\
0 & 0 & 0 & -1 & 0 & 1 \\
0 & 0 & 0 & 1 & 0 & -1
\end{array}\right]
$$

By Gauss-elimination, this matrix can be transformed to the upper-triangular matrix

$$
\left[\begin{array}{rrrrrr}
0 & -1 & 0 & -1 & 0 & -1 \\
0 & 0 & 0 & -1 & 0 & 1 \\
0 & 0 & 0 & 0 & 0 & 0 \\
0 & 0 & 0 & 0 & 0 & 0
\end{array}\right]
$$

Therefore, the integer vectors $n^{\mathbb{1}}, n^{2}$ and $n^{3}$ given by

$$
n^{1}:=[1,-2,0,1,0,1], n^{2}:=[0,-2,1,1,0,1], n^{3}:=[0,-2,0,1,1,1]
$$

form a basis for $\operatorname{Ker}\left(\left(M^{+}\right)^{t}\right)$.

For every assessment $(\sigma, \beta)$ we have by definition

$$
b_{x}^{+}(\beta)=\log \beta(x)-\log \beta(r(x))
$$


and

$$
s_{x}^{+}(\sigma)=\sum_{a \in A^{+} \cap A_{x}} \log \sigma(a)-\sum_{a \in A^{+} \cap A_{r(\approx)}} \log \sigma(a)
$$

for every $x \in X$. Hence, $b_{x}^{+}(\beta)=s_{x}^{+}(\sigma)=0$ if $x \in\left\{x_{1}, y_{1}, z_{2}\right\}$. Therefore, the equations $n^{i} \cdot b^{+}(\beta)=n^{i} \cdot s^{+}(\sigma)$ are all equivalent to the equation $n^{1} \cdot b^{+}(\beta)=$ $n^{1} \cdot s^{+}(\sigma)$. This equation is given by

$-2\left(\log \beta\left(x_{2}\right)-\log \beta\left(x_{1}\right)\right)+1\left(\log \beta\left(y_{2}\right)-\log \beta\left(y_{1}\right)\right)+1\left(\log \beta\left(z_{2}\right)-\log \beta\left(z_{1}\right)\right)=0$ since $s_{x}^{+}(\sigma)=0$ for all $x \in X$. If we take the exponential function on both sides, we obtain the equation

which is equivalent to

$$
\frac{\beta\left(x_{2}\right)^{-2}}{\beta\left(x_{1}\right)^{-2}} \frac{\beta\left(y_{2}\right)}{\beta\left(y_{1}\right)} \frac{\beta\left(z_{2}\right)}{\beta\left(z_{1}\right)}=1
$$

$$
\beta\left(x_{1}\right)^{2} \beta\left(y_{2}\right) \beta\left(z_{2}\right)=\beta\left(x_{2}\right)^{2} \beta\left(y_{1}\right) \beta\left(z_{1}\right) .
$$

Finally, we may conclude that $\mathcal{A}^{c}\left(A^{+}, X^{+}\right)$is the set of assessments $(\sigma, \beta)$ with $A^{+}(\sigma)=A^{+}, X^{+}(\beta)=X^{+}$and

$$
\beta\left(x_{1}\right)^{2} \beta\left(y_{2}\right) \beta\left(z_{2}\right)=\beta\left(x_{2}\right)^{2} \beta\left(y_{1}\right) \beta\left(z_{1}\right) .
$$

\subsection{Structure of the set of consistent assess- ments}

In this section, we give a geometrical description of the set of consistent assessments. To this purpose, we use some results derived in the previous section.

Let $\Gamma$ be an extensive form game and the sets $A^{+}, X^{+}$be fixed. By Corollary 2.4.4 we know that either $\mathcal{A}^{c}\left(A^{+}, X^{+}\right)$is empty or $\mathcal{A}^{c}\left(A^{+}, X^{+}\right)$is the set of assessments $(\sigma, \beta)$ with $A^{+}(\sigma)=A^{+}, X^{+}(\beta)=X^{+}$and

$$
n^{i} \cdot b^{+}(\beta)=n^{i} \cdot s^{+}(\sigma)
$$

for all $i$. If we take the exponential function on both sides and use the definitions of $b^{+}(\beta)$ and $s^{+}(\sigma)$, the equation $n^{i} \cdot b^{+}(\beta)=n^{i} \cdot s^{+}(\sigma)$ is equivalent to the equation

$$
\frac{\prod_{x \in X^{+}} \beta(x)^{n_{x}^{i}}}{\prod_{x \in X^{+}} \beta(r(x))^{n_{x}^{i}}}=\frac{\prod_{x \in X^{+}}\left[\prod_{c \in C_{x}} \tau(c) \cdot \prod_{a \in A^{+} \cap A_{x}} \sigma(a)\right]^{n_{x}^{i}}}{\prod_{x \in X^{+}}\left[\prod_{c \in C_{r(x)}} \tau(c) \cdot \prod_{a \in A^{+} \cap A_{r(x)}} \sigma(a)\right]^{n_{\Psi}^{i}}} .
$$


This equation can be written in the form

$$
\prod_{x \in X} \beta(x)^{m m_{x}^{i}} \cdot \prod_{a \in A} \sigma(a)^{m_{a}^{i}}=c^{i} \cdot \prod_{x \in X} \beta(x)^{l_{x}^{i}} \cdot \prod_{a \in A} \sigma(a)^{l_{a}^{i}}
$$

where $c^{i}$ is a constant and $m_{x}^{i}, m_{a}^{i}, l_{x}^{i}, l_{a}^{i}$ are non-negative integers.

Let $m^{i}$ be the vector $\left.\left(\left(m_{a}^{i}\right)_{a \in A},\left(m_{x}^{i}\right)_{x \in X}\right)\right)$ and $l^{i}$ the vector $\left.\left(\left(l_{a}^{i}\right)_{a \in A}\left(l_{x}^{i}\right)_{x \in X}\right)\right)$. By $(\sigma, \beta)^{m}$ we denote the expression

$$
\prod_{a \in A} \sigma(\alpha)^{m_{\alpha}^{i}} \cdot \prod_{x \in X} \beta(x)^{m_{x}^{i}}
$$

Similarly for $(\sigma, \beta)^{i}$. Using this notation, equation (2.2) can be written in the form

$$
(\sigma, \beta)^{m^{i}}=c^{i} \cdot(\sigma, \beta)^{l^{i}}
$$

and we obtain the following theorem.

Theorem 2.5.1. For every pair $A^{+}, X^{+}$either $\mathcal{A}^{\mathrm{c}}\left(A^{+}, X^{+}\right)$is empty or $A^{c}\left(A^{+}, X^{+}\right)$is determined by finitely many equations of the form

$$
(\sigma, \beta)^{m^{i}}=c^{i} \cdot(\sigma, \beta)^{l^{i}}
$$

where $m^{i}$ and $l^{i}$ are non-negative integer vectors.

For a non-negative vector $y \in \mathbb{R}^{n}$, we denote by $\log v$ the vector in $\left(\mathbb{R}^{*}\right)^{n}$ obtained by taking the coordinatewise logarithm in $v$. (Note that $z_{i}=-\infty$ if $v_{i}=0$.) For a set $S \subset \mathbb{R}^{n}$ of non-negative vectors the set $\log S \subset\left(\mathbb{R}^{*}\right)^{n}$ is defined in the obvious way.

A set $C \subset\left(\mathbb{R}^{*}\right)^{n}$ is called a cone with vertex 0 if for every $c^{1}, c^{2} \in C$ and every $a, b>0$ we have that $a c^{1}+b c^{2} \in C$. We call a set $C \subset\left(\mathbb{R}^{*}\right)^{n}$ a cone if there is a vector $v \in \mathbb{R}^{n}$ and a cone $C^{\prime}$ with vertex 0 such that $C=v+C^{\prime}$. A set $L \subset \mathbb{R}^{n}$ is said to be a logarithmic cone if $\log L$ is a cone. Hence, a logarithmic cone can be transformed into a cone by taking the coordinatewise logarithm.

Theorem 2.5.2 The set of consistent assessments is the intersection of a finite product of simplices with a finite number of logarithmic cones.

Proof. For a given pair $A^{+}, X^{+}$we denote by $A\left(A^{+}, X^{+}\right)$the set of assessments $(\sigma, \beta)$ with $A^{+}(\sigma)=A^{+}$and $X^{+}(\beta)=X^{+}$. By Theorem 2.5.1 we know that $\mathcal{A}^{+}\left(A^{+}, X^{+}\right)$is either empty or is equal to the set of assessments in $A\left(A^{*}, X^{+}\right)$which satisfy finitely many equations of the form

$$
(\sigma, \beta)^{m i}=c^{i} \cdot(\sigma, \beta)^{l^{i}}
$$


Assume that $\mathcal{A}^{c}\left(A^{+}, X^{+}\right)$is not empty. For a non-negative vector $(\sigma, \beta)$ of the same size as an assessment we define $A^{+}(\sigma)$ and $X^{+}(\beta)$ in the obvious way. By $L\left(A^{+}, X^{+}\right)$we denote the set of non-negative vectors $(\sigma, \beta)$ with $A^{+}(\sigma)=A^{+}, X^{+}(\beta)=X^{+}$and satisfying equations (2.3).

Since the variables $\sigma(a), \beta(x)$ with $a \notin A^{+}, x \notin X^{+}$do not appear in these equations, the set $L\left(A^{+}, X^{+}\right)$can be written as

$$
L\left(A^{+}, X^{+}\right)=\{y>0 \mid B(\log y)=d\} \times\{0\}
$$

for some appropriate matrix $B$ and vector $d$. Here, 0 denotes the vector of zeroes corresponding to the restriction of $(\sigma, \beta)$ on $A^{0}(\sigma) \times X^{0}(\beta)$. It can be seen easily that $L\left(A^{+}, X^{+}\right)$is a logarithmic cone.

If we denote the set of all assessments by $\mathcal{A}$, we obtain

$$
\mathcal{A}^{c}=\mathcal{A} \cap\left[\bigcup_{A^{+}, X^{+}} L\left(A^{+}, X^{+}\right)\right] .
$$

Since $\mathcal{A}$ is a finite product of simplices, it follows that $\mathcal{A}^{c}$ is the intersection of a finite product of simplices with a finite number of logarithmic cones. ${ }^{3} \square$

In Blume and Zame (1994) it has been shown that the set of sequential equilibria is a semi-algebraic set. (A set is called semi-algebraic if it is the finite union of sets determined by a finite number of polynomial inequalities. These inequalities may be strict or non-strict.) Using our insights about consistent assessments, this result can be shown within a few lines.

Obviously, the sets $L\left(A^{+}, X^{+}\right)$in the proof of Theorem 2.5 .2 are semialgebraic sets. Since $\mathcal{A}$ is also semi-algebraic, it follows that the set of consistent assessments is semi-algebraic. We already know that the set of sequentially rational assessments is semi-algebraic since sequential rationality is equivalent to a finite number of polynomial inequalities. Hence, the set of sequential equilibria is the intersection of two semi-algebraic sets and is therefore semi-algebraic itself.

Corollary 2.5.3 The set of sequential equilibria is a semi-algebraic set.

\subsection{Coincidence of Bayesian consistency and consistency}

Finally, we are able to provide an algebraic characterization of the class of extensive form games in which Bayesian consistency and consistency of assess-

\footnotetext{
${ }^{3}$ We thank an anorymous referee for his suggestion which led to a much shorter proof.
} 
ments are equivalent. In this characterization we use a somewhat modified version of the matrix $M$ as constructed in the proof of Theorem 2.3.1.

For a given set $A^{+}$we denote by $H^{*}$ the collection of information sets which are not reached if actions outside $A^{+}$are played with probability zero. By $X^{*}$ we denote the set of nodes in $H^{*}$. Let $M^{\prime}$ be the matrix which is generated by $A^{+}$and $X^{+}:=X$ as described in the proof of Theorem 2.3.1 and let $M^{*}$ be the restriction of the matrix $M^{\prime}$ to the rows which correspond to nodes in $X^{*}$. Moreover, we define the number

$$
n\left(A^{+}\right):=\left|X^{*}\right|-\left|H^{*}\right| .
$$

Lemma 2.6.1 Let the set $A^{+}$be given and let $M^{*}$ be the corresponding matrix constructed above. Then, every Bayesian consistent assessment $(\sigma, \beta)$ with $A^{+}(\sigma)=A^{*}$ is consistent if and only if

(1) $\operatorname{Ker}\left(M^{*}\right)$ contains a strictly negative vector and

(2) $M^{*}$ has rank $n\left(A^{+}\right)$.

Roughly speaking, each row in the matrix $M^{*}$ reveals the actions which lead to the corresponding node. Therefore, the rows in the matrix reflect the information structure in the game tree. If some rows are linearly dependent, it follows that the information structure imposes a restriction on the set of possible consistent beliefs at information sets reached with probability zero. The second condition in Lemma 2.6 .1 states that all rows in $M^{*}$, except the zero rows, are linearly independent, which means that the information structure puts no restrictions on the possible consistent beliefs at information sets which are reached with probability zero.

It is much harder to give an intuitive explanation of the first condition. By exploiting the construction of the matrix $M^{*}$, one can show that this condition is equivalent to saying that we can put misitake probabilities on the actions outside $A^{+}$such that all nodes in the zero probability information sets have the same mistake probability.

Proof. " $\Rightarrow$ " Let every Bayesian consistent assessment $(\sigma, \beta)$ with $A^{+}(\sigma)=$ $A^{+}$be consistent. Let $z=\left(z_{x}\right)_{x \in X}$ be an arbitrary real vector with $z_{x}=0$ for every $x=r(x)$. Since in a Bayesian consistent assessment $(\sigma, \beta)$ with $A^{+}(\sigma)=A^{+}$the beliefs at information sets in $H^{*}$ can be chosen freely, we can find a Bayesian comsistent assessment $(\sigma, \beta)$ with $A^{+}(\sigma)=A^{+}$such that $X^{+}(\beta) \supset X^{*}$ and

$$
b_{x}^{+}=s_{x}^{+}+z_{x}
$$

for all $x \in X^{*}, x \neq r(x)$. By assumption, $(\sigma, \beta)$ is even consistent. Using Lemma 2.3 .4 , we know that $b^{+} \in s^{+}+\operatorname{Im}\left(M^{+}\right)$and there is a vector $w<0$ 
with $M^{+} w=0$. The latter implies that $M^{*} w=0$, so $\operatorname{Ker}\left(M^{*}\right)$ contains a strictly negative vector. Moreover, the fact that $b^{+}-s^{+} \in \operatorname{Im}\left(M^{+}\right)$implies that $z \in \operatorname{Im}\left(M^{*}\right)$. Since this holds for every $z$ with $z_{x}=0$ for each $x=r(x)$, we have that $\operatorname{dim}\left(\operatorname{Im}\left(M^{*}\right)\right)=n\left(A^{+}\right)$. Therefore, $M^{*}$ has rank $n\left(A^{+}\right)$.

" $\Leftarrow$ " Let the conditions (1) and (2) in Lemma 2.6 .1 be satisfied and let $(\sigma, \beta)$ be a Bayesian consistent assessment with $A^{+}(\sigma)=A^{+}$and $X^{+}(\beta)=X^{+}$. Let $M$ be the matrix generated by $A^{+}$and $X^{+}$.

Condition (2) implies that $\operatorname{Im}\left(M^{*}\right)=\left\{\left(z_{x}\right)_{x \in X^{*}} \mid z_{x}=0\right.$ if $\left.x=r(x)\right\}$. It follows that there is a vector $y$ with

$$
(M y)_{x}=b_{x}^{+}-s_{x}^{+}
$$

for every $x \in X^{+}$. Hence, condition (2) in Lemma 2.3.4 is satisfied.

By assumption, $\operatorname{Ker}\left(M^{*}\right)$ contains a strictly negative vector $w^{1}$, so

$$
\left(M w^{1}\right)_{x}=0 \text { for every } x \in X^{*} .
$$

For every $x \in X^{+} \backslash X^{*}$ we have that $A_{x} \cap A^{0}=\emptyset$ and $A_{r(x)} \cap A^{0}=\emptyset$ since $(\sigma, \beta)$ is Bayesian consistent, so the row in $M$ corresponding to $x$ consists only of zero's if $x \in X^{+} \backslash X^{*}$. Therefore,

$$
\left(M w^{1}\right)_{x}=0 \text { for every } x \in X^{+} \backslash X^{*} .
$$

For every $x \in X^{0} \backslash X^{*}$ it holds that $A_{x} \cap A^{0} \neq \emptyset$ and $A_{r(x)} \cap A^{0}=\emptyset$ since $(\sigma, \beta)$ is Bayesian consistent, so the row in $M$ corresponding to $x$ consists only of 1 's and zero's and contains at least one 1 . Since $w^{1}$ is strictly negative, it follows that

$$
\left(M w^{1}\right)_{x}<0 \text { for every } x \in X^{0} \backslash X^{*} .
$$

Together with equations (2.4) and (2.5) we obtain

$$
\left(M w^{1}\right)_{x} \begin{cases}=0 & \text { if } x \in X^{*} \text { or } x \in X^{+} \\ <0 & \text { if } x \in X^{0} \backslash X^{*} .\end{cases}
$$

Since $\operatorname{Im}\left(M^{*}\right)=\left\{\left(z_{x}\right)_{x \in X^{*}} \mid z_{x}=0\right.$ if $\left.x=r(x)\right\}$ we can find a vector $w^{2}$ such that

$$
\left(M w^{2}\right)_{x}=\left\{\begin{array}{rc}
0 & \text { if } x \in X^{+} \cap X^{*} \\
-1 & \text { if } x \in X^{0} \cap X^{*} .
\end{array}\right.
$$

We already know that for every $x \in X^{+} \backslash X^{*}$ the row in $M$ corresponding with $x$ consists only of zero's, so $\left(M w^{2}\right)_{x}=0$ for every $x \in X^{+} \backslash X^{*}$. Hence, it follows that

$$
\left(M w^{2}\right)_{x}=\left\{\begin{aligned}
0 & \text { if } x \in X^{+} \\
-1 & \text { if } x \in X^{0} \cap X^{*} .
\end{aligned}\right.
$$


Combing equations (2.6) and (2.7) and using the fact that $w^{1}$ is strictly negative leads to the conclusion that wre can find a $k \in \mathbb{N}$ such that $k w^{1}+w^{2}$ is strietly negative and

$$
\left(M\left(k w^{1}+w^{2}\right)\right)_{x} \begin{cases}=0 & \text { if } x \in X^{4} \\ <0 & \text { if } x \in X^{0}\end{cases}
$$

If we define $w:=k w^{1}+w^{2}$ we have that $w<0, M^{+} w=0$ and $M^{0} w<0$. Therefore, condition (1) in Lemma 2.3 .4 is also satisfied which implies that $(\sigma, \rho)$ is consistent.

With the help of this lemma, it is easy to give the following algebraic characterization of the class of extensive form games in which Bayesian consistency and consistency of assessments are the same.

Theorem 2.6.2 In an extensive form game each Bayesian consistent assessment is consistent if and only if for every $A^{+}$the corresponding matrix $M^{*}$ has rank $n\left(A^{+}\right)$and $\operatorname{Ker}\left(M^{*}\right)$ contains a strictly negative vector.

This result can be used to show that Bayesian consistency and consistency are equivalent in every finite signaling game.

The problem of finding special classes of extensive form games in which the definition of sequential equilibrium is equivalent to a weaker definition (without sequences and limits) is also investigated by Fudenberg and Tirole (1991) and Batigalli (1996). Fudenberg and Tirole show that sequential equilibria and perfect Bayesian equilibria coincide in some special classes of multi-period games with observed actions. In Batigalli, it is shown that in games with observable deviators, consistency is equivalent to an independence property for conditional probability systems on the space of strategy profiles. 


\section{Chapter 3}

\section{Consistency of assessments in infinite signaling games}

\subsection{Introduction}

This chapter is based on Perea y Monsuwé et al. (1996a).

One of the most widely applied classes of games in economics is the class of signaling games. A signaling game is a game of incomplete information where two players are involved; player 1 - the sender - moves first and sends a message to player 2 - the receiver - who observes the message and chooses an answer. Player 1 has more information than player 2, which is modeled by assuming that player 1's type is drawn by a move of nature at the beginning of the game, player 1 is informed about the outcome of this draw, but player 2 is not; the distribution of nature's move, however, is common knowledge. Player 1's message may serve as a signal to convey or hide information about player 1's type. Think for instance of player 1 as the seller of a car who is informed about the quality of the car, and of player 2 as the uninformed potential buyer who is to say yes or no to a sales contract specifying the price as well as warranty conditions.

Signaling games - and, more generally, dynamic games of imperfect or incomplete information - are analyzed by considering not only the strategies of the players but also the beliefs that an uninformed player may have at his information sets. In a signaling game as described above, an assessment is a pair of strategies together with a probability distribution (beliefs) assessed by player 2 over the possible types of player 1 . Common to all kinds of Nash equilibrium refinements considered in the literature on signaling games is the sequential rationality requirement which says that the players' strategies are best responses to each other, where player 2 maximizes his payoff given his 
beliefs. Moreover, these beliefs must be consistent with Bayes' rule whenever possible - i.e., at all information sets reached with positive probability, where these probabilities depend on the distribution of nature's move and player 1 's strategy. The latter property is called Bayesian consistency.

In a finite signaling game, an assessment is called a sequential equilibrium if it satisfies sequential rationality and Bayesian consistency (see Cho and Kreps (1987)).

An essential part in the definition of sequential equilibria for general (finite) extensive form games, as introduced by Kreps and Wilson (1982), is the consistency condition. Roughly, this means that the assessment can be approximated by a sequence of Bayesian consistent and completely mixed assessments. This condition is a kind of 'trembling hand' condition: the beliefs of a player should be consistent, in the limit, with the beliefs he would have according to Bayesian updating if all players would 'tremble', so that each of his information sets would be reached with positive probability. Just as is the case with perfect equilibria, the requirement is that beliefs be consistent with some trembles, not with all trembles.

Moreover, consistency of assessments can be viewed as a condition which requires the beliefs to reflect and respect the structure of information sets in the game. By the structure of the information sets we mean the positions of the information sets in the game tree and the way different information sets are connected via actions and chance moves. In infinite extensive form games, such as infinite signaling games, the collection of information sets may be (uncountably) infinite. However; similarly to finite extensive form games, information sets in infinite extensive form games have specific positions in the (possibly infinite) game tree and are connected in a specific way. This is the reason why we think that the idea of consistency is also meaningful in infinite extensive form games.

It is well known that consistency is equivalent to Bayesian consistency in finite signaling games. This is why the definition of sequential equilibria for finite signaling games uses Bayesian consistency instead of consistency.

In this chapter, we try to apply this idea of consistency in infinite signaling games where the sets of types, messages and answers are complete separable metric spaces. In the last few years, a lot of attention has been paid to infinite signaling games. See, for instance, Mailath $(1987,1988)$ and Manelli (1994), to name just a few. In contrast to finite extensive form games, it is not obvious how to define completely mixed assessments and convergence of assessments in infinite signaling games. Since the concept of consistency depends on the way in which these two concepts are defined, a whole variety of definitions for consistency is possible.

A natural requirement for a consistency concept is that it should imply 
Bayesian consistency. This requirement is called condition A. It turns out that this condition heavily restricts the number of convergence concepts for assessments that can be used in the consistency concept. An example will show that weak convergence of probability measures is too weak for this purpose. Therefore, we have to use a stronger kind of convergence, which we will call pointwise convergence of probability measures.

In order to stay close to the original definition of consistency for finite extensive form games, in an appropriate consistency concept almost all local strategies and beliefs in the supporting sequence of assessments should converge pointwise to the original local strategies and beliefs. We call this restriction condition C.

In a finite extensive form game, a completely mixed assessment induces a positive probability on every node in the tree. If we consider an infinite signaling game as a tree where the number of nodes may be (uncountably) infinite, a completely mixed assessment should induce a positive probability on every non-empty, open set of nodes in the tree. This requirement is formalized in condition $\mathrm{B}$.

We call a consistency concept appropriate if it satisfies the three conditions $\mathrm{A}, \mathrm{B}$ and $\mathrm{C}$.

After introducing infinite signaling games in section 2 , we formulate the conditions $A, B$ and $C$ in section 3 and explain why these conditions are sensible. In section 4, a particular consistency concept, called strong consistency, is introduced and is shown to be appropriate.

In addition, we provide a characterization of strongly consistent assessments in section 5. This characterization states that an assessment is strongly consistent if and only if it is Bayesian consistent and the local beliefs following messages which lie isolated in the message space are absolutely continuous with respect to the a-priori probability distribution on the types. This characterization plays a crucial role in the remainder of the chapter. A consequence of this characterization is, for example, the observation that strong consistency and Bayesian consistency are equivalent in signaling games where the message space contains no isolated points or the type space is discrete.

In section 6 we show that the conditions A, B and C imply the conditions in this characterization. As a consequence, every appropriate consistency concept in infinite signaling games is a refinement of strong consistency. Moreover, we provide an example which shows how strong consistency can exclude some sequential equilibria in signaling games where strong consistency is not equivalent to Bayesian consistency.

In section 7 we give a definition of structurally consistent assessments in infinite signaling games which is, from our viewpoint, a natural implication of the original idea of structural consistency as given by Kreps and Wilson 
(1982). We conclude this chapter with a characterization of the class of structurally consistent assessments.

\title{
3.2 Model and definitions
}

\author{
Notation
}

For a metric space $X, M(X)$ is the set of all finite measures on $X$ and $\mathcal{P}(X)$ denotes the set of probability measures on $X$. By $\delta_{x}$ we denote the Diracmeasure on the point $x \in X$. For an $\varepsilon>0$ and an $x \in X$ the $\varepsilon$-neigbourhood of $x$ is denoted by $U_{\varepsilon}(x)$. Furthermore, by $a \vee b$ we denote the maximum of two numbers $a$ and $b$.

A metric space $X$ is called separable if it contains a countable dense subset. A Borel subset of $X$ is denoted by $X_{B}$.

In this section, we present the model of an infinite signaling game. This model, which is very similar to that of Manelli (1994), is in our view a natural extension of the finite model.

\author{
Infinite signaling games
}

An infinite signaling game (from now on simply called signaling game) is a sextuple $\left(T, M, A, u_{1}, u_{2}, \tau\right)$ where $T, M$ and $A$ are complete, separable metric spaces, $u_{1}$ and $u_{2}$ are measurable real functions on $T \times M \times A$ and $\tau$ is a strictly positive probability measure on $T$ (i.e., $\tau$ puts a positive weight on every non-empty, open subset of $T$ ).

The game is played according to the following rules: First, player 1's type is determined by nature according to the a-priori distribution $\tau$. After observing his type, player 1 sends a message $m \in M$. Being ignorant of player 1 's type, player 2 responds to this message with an answer $a \in A$. Finally, the payoffs for both players are given by $u_{1}(t, m, a)$ and $u_{2}(t, m, a)$, respectively. 


\section{Strategies and beliefs}

A behavior strategy for player 1 is a mapping $\sigma_{1}: T \rightarrow \mathcal{P}(M)$, such that the function $t \mapsto \sigma_{1}(t)\left(M_{B}\right)$ is measurable on $T$ for every $M_{B}$. So, for every type $t, \sigma_{1}(t)$ defines a probability measure on the message space $M$.

A behavior strategy for player 2 is a mapping $\sigma_{2}: M \rightarrow \mathcal{P}(A)$, such that the function $m \mapsto \sigma_{2}(m)\left(A_{B}\right)$ is measurable on $M$ for every $A_{B}$. For every message $m, \sigma_{2}(m)$ defines a probability measure on the answer space $A$. The probability measures $\sigma_{1}(t)$ and $\sigma_{2}(m)$ are called local strategies. A pair $\sigma=\left(\sigma_{1}, \sigma_{2}\right)$ is called a behavior strategy profile (BSP).

A belief system is a mapping $\beta: M \rightarrow \mathcal{P}(T)$, such that the function $m \mapsto$ $\beta(m)\left(T_{B}\right)$ is measurable for every $T_{B}$.

For a message $m$ and Borel set $T_{B}, \beta(m)\left(T_{B}\right)$ can be interpreted as the (subjective) probability that player 2 assigns to the event that player 1 has a type in $T_{B}$ if he observes the message $m$. The probability measures $\beta(m)$ are called local beliefs.

\section{Sequential rationality and Bayesian cansistency}

A pair $(\sigma, \beta)$ is called an assessment. An assessment $(\sigma, \beta)$ is called sequentially rational if the local strategy $\sigma_{1}(t)$ maximizes player 1's expected payoff for every $t \in T$ and if at every message $m$, the local strategy $\sigma_{2}(m)$ maximizes player 2's expected payoff given his beliefs about player 1's type. Formally: if for every $t \in T$ and $\mu \in \mathcal{P}(M)$

$$
\int_{A} \int_{M} u_{1}(t, m, a) d \sigma_{1}(t) d \sigma_{2}(m) \geq \iint_{A} u_{1}(t, m, a) d \mu d \sigma_{2}(m)
$$

and if for every $m \in M$ and $\alpha \in \mathcal{P}(A)$

$$
\iint_{A} u_{2}(t, m, a) d \beta(m) d \sigma_{2}(m) \geq \iint_{A} u_{2}(t, m, a) d \beta(m) d \alpha .
$$

Note that $\sigma_{1}(t)$ in the first integral does not mean that we integrate over $t$. In this integral, $t$ is fixed, and we integrate over $m$ with respect to the probability measure $\sigma_{1}(t)$.

A BSP $\sigma$ induces the probability measure $\pi^{\sigma}$ on $T \times M$ defined by

$$
\pi^{\sigma}\left(T_{B} \times M_{B}\right):=\int_{T_{B}} \sigma_{1}(t)\left(M_{B}\right) d \tau
$$


Hence, $\pi^{\sigma}\left(T_{B} \times M_{B}\right)$ is the probability that player 1 has a type in $T_{B}$ and sends a message in $M_{B}$ given the fact that $\sigma$ is played.

By $P^{\sigma}$ we denote the marginal distribution on $M$ corresponding to $\pi^{\sigma}$. Hence,

$$
P^{\sigma}\left(M_{B}\right)=\int_{T} \sigma_{1}(t)\left(M_{B}\right) d \tau
$$

is the probability that a message in $M_{B}$ will be sent if $\sigma$ is played.

An assessment $(\sigma, \beta)$ is called Bayesian consistent if $\beta$ is a conditional distribution for $\pi^{\sigma}$. This means that for all $T_{B} \times M_{B}$

$$
\int_{M_{B}} \beta(m)\left(T_{B}\right) d P^{\sigma}=\pi^{\sigma}\left(T_{B} \times M_{B}\right)
$$

In finite extensive form games, Bayesian consistency completely determines the beliefs at all information sets which are reached with positive probability. The crucial difference between Bayesian consistency in finite extensive form games and Bayesian consistency in infinite signaling games lies in the fact that in infinite signaling games, it puts restrictions on the behavior of beliefs at collections of information sets rather than at individual information sets.

An assessment $(\sigma, \beta)$ is called a sequential equilibrium if it is sequentially rational and Bayesian consistent. However, a sequential equilibrium does not always exist, as is shown by an example of van Damme (1987).

\subsection{Minimal requirements for consistency con- cepts}

As mentioned before, we investigate several possibilities to define consistency of assessments in infinite signaling games. Of course, there are a lot of different ways to do this, but not all of them are equally meaningful. In order to decide whether a given consistency concept is appropriate, we develop a system of minimal requirements which such a concept should satisfy.

Formally speaking, a consistency concept is a mapping $\varphi$ which assigns to every signaling game $\Gamma$ a set $\varphi(\Gamma)$ of assessments. An assessment in $\varphi(\Gamma)$ is called consistent w.r.t. $\varphi$.

An appropriate consistency concept should, from our point of view, reflect the idea of consistency as it was defined by Kreps and Wilson (1982) for finite extensive form games. This requirement can be formalized by saying that a consistency concept $\varphi$ should have the following canonical form: 
"An assessment $(\sigma, \beta)$ is consistent w.r.t. $\varphi$ if and only if there is a sequence $\left(\sigma^{k}, \beta^{k}\right)_{k \in \mathbb{N}}$ of Bayesian consistent assessments such that

(1) $\left(\sigma^{k}, \beta^{k}\right)$ is completely mixed for every $k$ and

(2) $\left(\sigma^{k}, \beta^{k}\right)_{k \in \mathbb{N}}$ converges to $(\sigma, \beta) .{ }^{m}$

This canonical form does not induce a unique consistency concept since it depends on the way we define completely mixed assessments and convergence of assessments. The particular definitions of completely mixed assessments and convergence of assessments used in $\varphi$ are called complete mixedness w.r.t. $\varphi$ and convergence w.r.t. $\varphi$, respectively.

However, we will not regard every concept $\varphi$ satisfying this canonical form as appropriate, for reasons to become clear later. More precisely, we will impose further requirements labeled as conditions $A, B$ and $C$.

\section{Consistency should imply Bayesian consistency}

First of all, an appropriate consistency concept should always imply Bayesian consistency.

Condition A. Every assessment which is consistent w.r.t. $\varphi$ should be Bayesian consistent.

This condition may seem easy to fulfill but, as we show later on in this section, we need a rather strong convergence concept in order to satisfy this requirement. Weak convergence of probability measures, for example, is not strong enough for this purpose.

\section{Restriction on completely mixed assessments}

Next, we put a restriction on the definition of completely mixed assessments. In Selten's article about perfect equilibria (1975), completely mixed behavior strategies are used because they induce a positive probability on every node in the tree of a finite extensive form game.

A signaling game can also be interpreted as an extensive form game with a possibly infinite number of nodes. The nodes which follow the actions of player $I$ are given by pairs $(t, m) \in T \times M$. Of course, in general it is not possible to require that player 1's strategy induces a positive probability on every single node in $T \times M$. This condition can not be satisfied if, for example, $T \times M$ is uncountable. 
However, Simon and Stinchcombe (1995) discuss a very natural way to define completely mixed strategies in the infinite case. They call a mixed strategy in an infinite normal form game of full support if it puts positive wreight on every non-empty, open subset of pure strategies.

Combining the ideas of both articles, we arrive at the following condition which says that a completely mixed assessment in a signaling game should always induce a positive probability on every non-empty, open subset of nodes $(t, m)$.

Condition B. Every assessment $(\sigma, \beta)$ which is completely mixed w.r.t. $\varphi$ should have the property that

$$
\int_{T_{B}} \sigma_{1}(t)\left(M_{B}\right) d \tau>0
$$

for every non-empty, open subset $\left(T_{B}, M_{B}\right) \in T \times M$.

A possible way to define completely mixed assessments is by saying that an assessment $(\sigma, \beta)$ is completely mixed if the local strategy $\sigma_{1}(t)$ is a strictly positive probability measure on $M$ for every $t$. We call such assessments pointwise completely mixed. Obviously, this definition satisfies condition B.

An important property of completely mixed assessments in finite extensive form games is the fact that they always induce strictly positive beliefs at every information set if the assessment satisfies Bayesian consistency. In an infinite signaling game, an assessment which is pointwise completely mixed and Bayesian consistent does not necessarily have the property that the local belief $\beta(m)$ is strictly positive for every $m$. However, a similar but somewhat weaker property can be shown. It turns out that for every non-empty, open subset $T_{B 3}$ the set $\left\{m \mid \beta(m)\left(T_{B}\right)>0\right\}$ is dense in $M$.

Why is weak convergence not strong enough?

A possible convergence concept which can be used in a consistency concept is the so-called weak convergence of assessments. We say that a sequence $\left(\sigma^{k}, \beta^{k}\right)_{k \in \mathbb{N}}$ of assessments converges weakly to an assessment $(\sigma, \beta)$ if $\left(\sigma_{1}^{k}(t)\right)_{k \in \mathbb{N}}$ converges weakly to $\sigma_{1}(t)$ for every $t,\left(\sigma_{2}^{k}(m)\right)_{k \in \mathbb{N}}$ converges weakly to $\sigma_{2}(m)$ for every $m$ and $\left(\beta^{k}(m)\right)_{k \in \mathbb{N}}$ converges weakly to $\beta(m)$ for every $m$. Although weak convergence seems a very natural convergence concept in this situation, the following example shows that even the consistency concept which makes use of weak convergence and pointwise completely mixed 
assessments does not imply Bayesian consistency and can therefore not be appropriate.

Example 3.1. Let $\varphi$ be the consistency concept induced by pointwise complete mixedness and weak convergence. Let $\Gamma$ be a signaling game in which $T=M=[0,1]$ and $\tau$ is the uniform distribution on $T$. We denote the uniform distribution on $[0,1]$ by $u$, and for an interval $I \subset[0,1]$ the uniform distribution on $I$ is denoted by $u_{\Gamma}$.

Now, we construct an assessment $(\sigma, \beta)$ which is consistent w.r.t. $\varphi$ but not Bayesian consistent.

Let the behavior strategy $\sigma_{1}$ and the belief system $\beta$ be given by

$$
\begin{aligned}
& \sigma_{1}(t):=u \text { for every } t, \\
& \beta(m):= \begin{cases}\frac{1}{3} u_{\left[0, \frac{1}{2}\right]}+\frac{2}{3} u_{\left[\frac{1}{2}, 2\right]} & \text { if } m \notin \mathbb{Q} \\
u_{\left[0, \frac{1}{2}\right]} & \text { if } m \in \mathbb{Q} .\end{cases}
\end{aligned}
$$

Furthermore, we choose an arbitrary behavior strategy $\sigma_{2}$ for player 2 .

The assessment $(\sigma, \beta)$ is not Bayesian consistent since $\int_{[0,1]} \beta(m)\left(\left[0, \frac{1}{2}\right]\right) d u=$ $\frac{1}{3}$ but $\int_{\left[0, \frac{1}{2}\right]} \sigma_{1}(t)([0,1]) d u=\frac{1}{2}$.

In order to show that $(\sigma, \beta)$ is consistent w.r.t. $\varphi$, we construct a sequence $\left(\sigma^{k}, \beta^{k}\right)_{k \in \mathbb{N}}$ of assessments which are Bayesian consistent and pointwise completely mixed such that $\left(\sigma^{k}, \beta^{k}\right)_{k \in \mathbb{N}}$ converges weakly to $(\sigma, \beta)$.

For every $k \in \mathbb{N}$, let $M^{k}:=\left\{0, \frac{1}{k}, \frac{2}{k}, \ldots, \frac{k-1}{k}\right\}$ and let $\mu^{k}$ be the probability measure on $M$ which puts probability $\frac{1}{k}$ on every point in $M^{k}$. It can be shown that the sequence $\left(\mu^{k}\right)_{k \in \mathbb{N}}$ converges weakly to $u$. (See, for example, Example 25.3 in Billingsley (1986).)

For every $k$, we define $\sigma_{1}^{k}$ and $\beta^{k}$ by

$$
\begin{aligned}
\sigma_{1}^{k}(t): & = \begin{cases}\frac{1}{2} u+\frac{1}{2} \mu^{k} & \text { if } t \leq \frac{1}{2} \\
u & \text { if } t>\frac{1}{2}\end{cases} \\
\beta^{k}(m): & = \begin{cases}\frac{1}{3} u_{\left[0, \frac{1}{2}\right]}+\frac{2}{3} u_{\left[\frac{1}{2}, 1\right]} & \text { if } m \notin M^{k} \\
u_{\left[0, \frac{1}{2}\right]} & \text { if } m \in M^{k} .\end{cases}
\end{aligned}
$$

Obviously, $\sigma_{1}^{k}(t)$ is strictly positive for every $t$, and the sequence $\left(\sigma_{1}^{k}(t)\right)_{k \in \mathbb{N}}$ converges weakly to $\sigma_{1}(t)$ for every $t$. In order to show that $\left(\beta^{k}(m)\right)_{k \in \mathbb{N}}$ converges weakly to $\beta(m)$ for all $m$, we distinguish two cases.

If $m \notin \mathbb{Q}$, it follows that $m \notin M^{k}$ for every $k$. By construction, $\beta^{k}(m)=\beta(m)$ for all $k$, which implies that $\beta^{k}(m)$ converges weakly to $\beta(m)$.

For the case $m \in \mathbb{Q}$, we need the following lemma, which is formulated as Theorem 2.3 in Billingsley (1968). 
Lemma 3.3.1 Let $X$ be a metric space and $\mu, \mu^{1}, \mu^{2}, \ldots$ probability measures on $X$. Then, the sequence $\left(\mu^{k}\right)_{k \in \mathrm{N}}$ converges weakly to $\mu$ if and only if every subsequence of $\left(\mu^{k}\right)_{k \in \mathbb{M}}$ contains a further subsequence which converges weakly to $\mu$.

Now, let $m \in \mathbb{Q}$ and $\left(\beta^{k^{k}}(m)\right)_{k \in \mathbb{N}}$ a subsequence of $\left(\beta^{k}(m)\right)_{k \in \mathbb{N}}$. We can find a further subsequence $\left(\beta^{k^{\prime \prime}}(m)\right)_{k \in N}$ such that $m \in M^{k^{\prime \prime}}$ for every $k^{\prime \prime}$, which implies that $\beta^{k^{\prime \prime}}(m)=\beta(m)$ for every $k^{\prime \prime}$. It follows that $\left(\beta^{k^{\prime \prime}}(m)\right)_{k \in \mathbb{N}}$ converges weakly to $\beta(m)$. By Lemma 3.3.1 $\left(\beta^{k}(m)\right)_{k \in \mathbb{N}}$ converges weakly to $\beta(m)$.

Finally, it can be shown that $\left(\sigma^{k}, \beta^{k}\right)$ is Bayesian consistent for every $k$. This implies that $(\sigma, \beta)$ is consistent w.r.t. $\varphi$.

From the above example, we learn that the 'weakness' of weak convergence lies in the fact that the expected value of a bounded function w.r.t. a weakly convergent sequence of probability measures only converges to the expected value w.r.t. the limit measure if this function is continuous. However, the functions which occur in signaling games are typically not continuous. Therefore, we introduce a sharpening of weak convergence, called pointwise convergence of probability measures, which preserves the expected value of every bounded (continuous or non-continuous) function in the limit.

\section{Pointwise convergence of probability measures}

Let $X$ be a metric space and $\left(\mu^{k}\right)_{k \in \mathbb{N}}$ a sequence of probability measures on $X$. We say that $\left(\mu^{k}\right)_{k \in \mathbb{N}}$ converges pointwise to a probability measure $\mu$ if

$$
\lim _{k \rightarrow \infty} \mu^{k}\left(X_{B}\right)=\mu\left(X_{B}\right)
$$

for every Borel set $X_{B}$.

Obviously, every pointwise convergent sequence is also weakly convergent, since weak convergence only requires the equation above to be true for Borel sets $X_{B}$ in which the boundary has measure zero under $\mu$.

If we consider the strong metric on probability measures given by

$$
d(\mu, \nu):=\sup \left\{\left|\mu\left(X_{B}\right)-\nu\left(X_{B}\right)\right| \mid X_{B} \text { measurable }\right\},
$$

it is clear that convergence w.r.t. the strong metric implies pointwise convergence.

The following lemma shows that pointwise convergence can also be defined by convergence of integrals of bounded and measurable functions. ${ }^{1}$

\footnotetext{
Whe thank Peter Wakker for this proof.
} 
Lemma 3.3.2 Let $X$ be a complete separable metric space and $\mu, \mu^{1}, \mu^{2}, \ldots$ probablity measures on $X$. Then $\left(\mu^{k}\right)_{k \in \mathbb{N}}$ converges pointwise to $\mu$ if and only if

$$
\lim _{k \rightarrow \infty} \int_{X} f d \mu^{k}=\int_{X} f d \mu
$$

for every bounded and measurable function $f$.

Proof. It can be shown easily that every sequence $\left(\mu^{k}\right)_{k \in \mathbb{N}}$ satisfying the condition in the lemma is pointwise convergent to $\mu$ by choosing $f$ equal to the indicator function on the Borel set $X_{B}$.

Now, let $\left(\mu^{k}\right)_{k \in \mathbb{N}}$ be pointwise convergent to $\mu$ and let $f$ be a bounded measurable function on $X$. W.l.o.g., we assume that $f: X \rightarrow(0,1)$. For every $k$, we define the sets

$$
\begin{aligned}
& A_{i}(k):=\left\{x \mid f(x) \geq \frac{i}{k}\right\}, \quad i=0, \ldots, k \text { and } \\
& B_{i}(k):=\left\{x \mid \frac{i-1}{k} \leq f(x)<\frac{i}{k}\right\}, \quad i=1, \ldots, k .
\end{aligned}
$$

By the definition of the integral, we have for every $k$

$$
\sum_{i=1}^{k} \frac{i-1}{k} \mu\left(B_{i}(k)\right) \leq \int_{X} f d \mu \leq \sum_{i=1}^{k} \frac{i}{k} \mu\left(B_{i}(k)\right)
$$

Since $\sum_{i=1}^{k} \frac{i-1}{k} \mu\left(B_{i}(k)\right)=\frac{1}{k} \sum_{i=1}^{k} \mu\left(A_{i}(k)\right)$ and $\sum_{i=1}^{k} \frac{i}{k} \mu\left(B_{i}(k)\right)=\frac{1}{k}+\frac{1}{k} \sum_{i=1}^{k} \mu\left(A_{i}(k)\right)$. it follows that

$$
\frac{1}{k} \sum_{i=1}^{k} \mu\left(A_{i}(k\rangle\right) \leq \int_{X} f d \mu \leq \frac{1}{k}+\frac{1}{k} \sum_{i=1}^{k} \mu\left(A_{i}(k)\right) .
$$

Similarly, we can show that

$$
\frac{1}{k} \sum_{i=1}^{k} \mu^{k}\left(A_{i}(k)\right) \leq \int_{X} f d \mu^{k} \leq \frac{1}{k}+\frac{1}{k} \sum_{i=1}^{k} \mu^{k}\left(A_{i}(k)\right) .
$$

These inequalities imply that

$$
\int_{X} f d \mu=\lim _{k \rightarrow \infty} \frac{1}{k} \sum_{i=1}^{k} \mu\left(A_{i}(k)\right)
$$

and

$$
\lim _{k \rightarrow \infty} \int_{X} f d \mu^{k}=\lim _{k \rightarrow \infty} \frac{1}{k} \sum_{i=1}^{k} \mu^{k}\left(A_{i}(k)\right)
$$


Therefore, it suffices to show that

$$
\lim _{k \rightarrow \infty} \frac{1}{k} \sum_{i=1}^{k} \mu^{k}\left(A_{i}(k)\right)=\lim _{k \rightarrow \infty} \frac{1}{k} \sum_{i=1}^{k} \mu\left(A_{i}(k)\right) .
$$

Assume that this would not be true. Then, w.l.o.g., there is a $\delta>0$ such that

$$
\left|\frac{1}{k} \sum_{i=1}^{k} \mu^{k}\left(A_{i}(k)\right)-\frac{1}{k} \sum_{i=1}^{k} \mu\left(A_{i}(k)\right)\right| \geq \delta \text { for all } k
$$

So, for every $k$ there is an integer $i_{k} \leq k$ such that $\left|\mu^{k}\left(A_{i_{k}}(k)\right)-\mu\left(A_{i_{k}}(k)\right)\right| \geq$ 8. W.1.0.g. we may assume that $\mu^{k}\left(A_{i_{k}}(k)\right) \geq \delta+\mu\left(A_{i_{k}}(k)\right)$ for every $k$.

Since $0 \leq \frac{i_{k}}{k} \leq 1$, the sequence $\frac{i_{k}}{k}$ contains a monotone convergent subsequence. W.1.o.g. we assume that the sequence $\frac{i_{k}}{k}$ is monotone and convergent.

Case $1: \frac{i_{k}}{k} \uparrow r$ for some $r \in[0,1]$. Then, by construction, $A_{i_{k+1}}(k+1) \subset A_{i_{k}}(k)$ for every $k$, and

$$
\cap_{k} A_{i_{k}}=A:=\{x \mid f(x) \geq r\} .
$$

Since $\mu^{l}\left(A_{i_{k}}(k)\right) \geq \mu^{l}\left(A_{i_{l}}(l)\right)$ for $l \geq k$, it follows that

$$
\begin{aligned}
\mu^{l}\left(A_{i_{k}}(k)\right) & \geq \delta+\mu\left(A_{i_{l}}(l)\right) \text { for } l \geq k \text { and therefore } \\
\lim _{l \rightarrow \infty} \mu^{l}\left(A_{i_{k}}(k)\right) & \geq \delta+\lim _{l \rightarrow \infty} \mu\left(A_{i_{1}}(l)\right) \text { for every } k .
\end{aligned}
$$

By assumption, $\lim _{l \rightarrow \infty} \mu^{l}\left(X_{B}\right)=\mu\left(X_{B}\right)$ for every Borel set $X_{B}$, so $\lim _{l \rightarrow \infty} \mu^{l}\left(A_{i_{k}}(k)\right)=$ $\mu\left(A_{i_{k_{k}}}(k)\right)$. Furthermore, by the monotone convergence theorem; $\lim _{l \rightarrow \infty} \mu\left(A_{i_{l}}(l)\right)=$ $\mu(A)$. Combining these facts leads to the conclusion that

$$
\mu\left(A_{i_{k}}(k)\right) \geq \delta+\mu(A) \text { for every } k \text {. }
$$

However, this implies that:

$$
\lim _{k \rightarrow \infty} \mu\left(A_{i_{k}}(k)\right) \geq \delta+\mu(A)
$$

which contradicts the fact that $\lim _{k \rightarrow \infty} \mu\left(A_{i_{k}}(k)\right)=\mu(A)$.

Case 2: $\frac{i_{k}}{k} \downarrow r$ for some $r \in[0,1]$. Then, by construction, $A_{i_{k+1}}(k+1) \supset A_{i_{k}}(k)$ for every $k$ and

$$
\cup_{k} A_{i_{k}}(k)=A:=\{x \mid f(x) \geq r\} .
$$


Since $\mu^{l}\left(A_{i_{k}}(k)\right) \geq \mu^{l}\left(A_{i_{l}}(l)\right)$ for $k \geq l$, it follows that

$$
\begin{aligned}
\mu^{l}\left(A_{i_{k}}(k)\right) & \geq \delta+\mu\left(A_{i_{l}}(l)\right) \text { for } k \geq l \text { and } \\
\lim _{k \rightarrow \infty} \mu^{l}\left(A_{i_{k}}(k)\right) & \geq \delta+\mu\left(A_{i_{l}}(l)\right) \text { for every } l .
\end{aligned}
$$

By the monotone convergence theorem, $\lim _{k \rightarrow \infty} \mu^{l}\left(A_{i_{\bar{k}}}(k)\right)=\mu^{l}(A)$. Together with the inequality above, we obtain $\mu^{l}(A) \geq \delta+\mu\left(A_{u_{i}}(l)\right)$ for every $l$, which implies that $\lim _{l \rightarrow \infty} \mu^{l}(A) \geq \delta+\lim _{l \rightarrow \infty} \mu\left(A_{i_{l}}(l)\right)$. However, this leads to a contradiction since $\lim _{l \rightarrow \infty} \mu^{l}(A)=\mu(A)$ and $\lim _{l \rightarrow \infty} \mu\left(A_{i_{l}}(l)\right)=\mu(A)$.

A similar characterization holds for weakly convergent sequences: the sequence $\left(\mu^{k}\right)_{k \in \mathbb{N}}$ converges weakly to $\mu$ if and only if the equation in the lemma is true for every bounded and continuous function $f$.

In the next section, we show that the pointwise convergence concept enables us to find an appropriate consistency concept.

\section{Restriction on convergence of assessments}

In view of the fact that strategies and beliefs in signaling games typically induce non-continuous functions, we regard pointwise convergence as a natural convergence concept to define consistency. As a minimal condition for an appropriate consistency concept, we require that almost all local strategies and local beliefs in the supporting sequence of assessments should converge pointwise to the local strategies and local beliefs in the limit assessment.

Condition C. Convergence of assessments w.r.t. $\varphi$ should be defined in such a way that, whenever $\left(\sigma^{k}, \beta^{k}\right)_{k \in \mathbb{N}}$ converges to $(\sigma, \beta)$ w.r.t. $\varphi$, there is a dense subset in $T$ such that $\left(\sigma_{1}^{k}(t)\right)_{k \in \mathbb{N}}$ converges pointwise to $\sigma_{1}(t)$ for every $t$ in this dense subset and $\left(\beta^{k}(m)\right)_{k \in \mathbb{N}}$ converges pointwise to $\beta(m)$ for every $m$ in some dense subset of $M$.

\section{Appropriate consistency concepts}

The last condition completes the framework which we use in our search for appropriate consistency concepts.

Definition. We call a consistency concept $\varphi$ appropriate if it has the canonical form and satisfies the conditions $A, B$ and $C$. 


\subsection{Strong consistency}

In this section, we present a particular consistency concept which we call strong consistency.

We call an assessment $(\sigma, \beta)$ strongly consistent if there is a sequence $\left(\sigma^{k}, \beta^{k}\right)_{k \in \mathbb{N}}$ of assessments which are Bayesian consistent and pointwise completely mixed, such that $\left(\sigma_{1}^{k}(t)\right)_{k \in \mathbb{N}}$ converges pointwise to $\sigma_{1}(t)$ for every $t$ and $\left(\beta^{k}(m)\right)_{k \in \mathbb{N}}$ converges pointwise to $\beta(m)$ for every $m$.

In order to show that this is an appropriate consistency concept, we only have to prove that strong consistency implies Bayesian consistency since it is clear it satisfies conditions $B$ and $C$. The proof of this fact is based on the following lemma.

Iuemma 3.4.1 Let $X$ be a complete, seperable metric space, $\left(\mu^{k}\right)_{k \in \mathbb{N}}$ a sequence of probability measures which converges pointwise to a probability measure $\mu$ and $\left(f^{k}\right)_{k \in \mathbb{N}}$ a sequence of measurable functions from $X$ to $[0,1]$ which converges pointwise to a measurable function $f$. Then $\lim _{k \rightarrow \infty} \int_{X} f^{k} d \mu^{k}=$ $\int_{X} f d \mu$

Proof. Let $\varepsilon>0$ be given. We can find a compact subset $K$ with $\mu(K)>$ $1-\varepsilon, \mu^{k}(K)>1-\epsilon$ for $k$ large enough and $\left|f^{k}(x)-f(x)\right|<\epsilon$ for all $x \in K$ and $k$ large enough. The latter follows from the fact that pointwise convergence of functions implies almost uniform convergence. Moreover, the pointwise convergence of $\left(\mu^{k}\right)_{k \in \mathbb{N}}$ implies that $\left|\int_{X} f d \mu^{k}-\int_{X} f d \mu\right|<\varepsilon$ for large $k$. But then, for large $k$,

$$
\begin{aligned}
\left|\int_{X} f^{k} d \mu^{k}-\int_{X} f d \mu\right| & \leq\left|\int_{X} f^{k} d \mu^{k}-\int_{X} f d \mu^{k}\right|+\left|\int_{X} f d \mu^{k}-\int_{X} f d \mu\right| \\
& \leq \int_{K}\left|f^{k}-f\right| d \mu^{k}+\int_{X \backslash K}\left|f^{k}-f\right| d \mu^{k}+\varepsilon \\
& \leq \varepsilon \cdot \mathbb{1}+\varepsilon+\varepsilon,
\end{aligned}
$$

which leads to the conclusion that $\lim _{k \rightarrow \infty} \int_{X} f^{k} d \mu^{k}=\int_{X} f d \mu$.

In order to show that strong consistency implies Bayesian consistency, we need one further lemma, which can be found as Exercise 18.25 (d) in Billingsley (1986).

Lemma 3.4.2 Let $T, M$ be metric spaces, $\beta$ a measurable on function $M, \tau$ a probability measure on $T, \sigma: T \rightarrow \mathcal{P}(M)$ such that the function $t \mapsto$ 
$\sigma(t)\left(M_{B}\right)$ is measurable for every $M_{B}$ and let the probability measure $P$ on $M$ be given by

$$
P\left(M_{B}\right):=\int_{T} \sigma(t)\left(M_{B}\right) d \tau \text { for every } M_{B}
$$

Then we have

$$
\int_{M} \beta(m) d P=\int_{T}\left[\int_{M} \beta(m) d \sigma(t)\right] d \tau .
$$

Lemma 3.4.3 Let $\left(\sigma^{k}, \beta^{k}\right)_{k \in \mathbb{A}}$ be a sequence of Bayesian consistent assessments such that $\left(\sigma_{1}^{k}(t)\right)_{k \in \mathbb{N}}$ converges pointwise to $\sigma_{1}(t)$ for every $t$ and $\left(\beta^{k}(m)\right)_{k \in \mathbb{N}}$ converges pointwise to $\beta(m)$ for every $m$. Then the assessment $(\sigma, \beta)$ is Bayesian consistent.

Proof. Let $T_{B}, M_{B}$ be Borel sets in $T$ and $M$ respectively. First, we show that

$$
\lim _{k \rightarrow \infty} \int_{M_{B}} \beta^{k}(m)\left(T_{B}\right) d P^{\sigma^{k}}=\int_{M_{B}} \beta(m)\left(T_{B}\right) d P^{\sigma}
$$

Using Lemma 3.4.2 we obtain

$$
\int_{M_{B}} \beta(m)\left(T_{B}\right) d P^{\infty}=\int_{T}\left[\int_{M_{B}} \beta(m)\left(T_{B}\right) d \sigma_{1}(t)\right] d \tau
$$

and

$$
\int_{M_{B}} \beta^{k}(m)\left(T_{B}\right) d P^{\sigma^{k}}=\int_{I}\left[\int_{M_{B}} \beta^{k}(m)\left(T_{B}\right) d \sigma_{1}^{k}(t)\right] d \tau .
$$

Since the functions $m \mapsto \beta^{k}(m)\left(T_{B}\right)$ are measurable functions from $M$ to $[0,1]$ converging pointwise to the function $m \mapsto \beta(m)\left(T_{B}\right)$, Lemma 3.4.1 implies

$$
\lim _{k \rightarrow \infty} \int_{M_{B}} \beta^{k}(m)\left(T_{B}\right) d \sigma_{1}^{k}(t)=\int_{M_{B}} \beta(m)\left(T_{B}\right) d \sigma_{1}(t)
$$

Since this holds for every $t$, it follows with the dominated convergence theorem that

$$
\begin{aligned}
\lim _{k \rightarrow \infty} \int_{M_{B}} \beta^{k}(m)\left(T_{B}\right) d P^{\sigma^{k}} & =\int_{T}\left[\int_{M_{B}} \beta(m)\left(T_{B}\right) d \sigma_{1}(t)\right] d \tau \\
& =\int_{M_{B}} \beta(m)\left(T_{B}\right) d P^{\sigma} .
\end{aligned}
$$

Furthermore, the functions $t \mapsto \sigma_{1}^{k}(t)\left(M_{B}\right)$ are measurable functions from $T$ to $[0,1]$ which converge pointwise to the function $t \mapsto \sigma_{1}(t)\left(M_{B}\right)$. By the dominated convergence theorem, we obtain

$$
\lim _{k \rightarrow \infty} \int_{T_{B}} \sigma_{1}^{k}(t)\left(M_{B}\right) d \tau=\int_{T_{B}} \sigma_{1}(t)\left(M_{B}\right) d \tau
$$


Combining these two results and using the fact that $\left(\sigma^{k}, \beta^{k}\right)$ is Bayesian consistent leads to the conclusion that

$$
\begin{aligned}
\int_{T_{B}} \sigma_{1}(t)\left(M_{B}\right) d \tau & =\lim _{k \rightarrow \infty} \int_{T_{B}} \sigma_{1}^{k}(t)\left(M_{B}\right) d \tau=\lim _{k \rightarrow \infty} \int_{M_{B}} \beta^{k}(m)\left(T_{B}\right) d P^{\sigma^{k}} \\
& =\int_{M_{B}} \beta(m)\left(T_{B}\right) d P^{\sigma} .
\end{aligned}
$$

Since this holds for arbitrary $T_{B}$ and $M_{B}$ it follows that $(\sigma, \beta)$ is Bayesian consistent.

From this lemma, it follows directly that strong consistency implies Bayesian consistency.

Corollary 3.4.4 Every strongly consistent assessment is Bayesian consistent.

This leads to the following conclusion.

Corollary 3.4.5 The strong consistency concept is appropriate.

\subsection{Characterization of strong consistency}

In this section, we give a characterization of strongly consistent assessments. Besides the fact that this characterization gives good insight into the structure of the set of strongly consistent assessments, it can be used later to show that every appropriate consistency concept is a refinement of strong consistency.

Before formulating this result, we first consider the topological structure of a separable metric space. We formulate several properties of such spaces in terms of the messtage set $M$.

A point $m \in M$ is called isolated if $\{m\}$ is an open subset of $M$. The set of all isolated points of $M$ is denoted by $M_{\text {iso }}$. Note that $m \in M_{\text {iso }}$ if and only if there is an $\varepsilon>0$ such that $M \cap U_{\varepsilon}(m)=\{m\}$. A point in $M$ that is not isolated is called an cccumulation point of $M$ and $M_{\text {aceu }}$ denotes the set of all accumulation points of $M$.

In the proof of Theorem 3.5 .2 we make use of the following property of seperable metric spaces.

Lemma 3.5.1 Let $M$ be a separable metric space and let $M^{*}$ be a countable dense subset of $M$. Then

(1) if $m \in M^{*} \cap M_{\text {accu, }}$ then $M^{*} \backslash\{m\}$ is a dense subset of $M$

(2) $M_{\text {iso }} \subset M^{*}$. 
Theorem 3.5.2 Let $\Gamma$ be a signaling game and let $(\sigma, \beta)$ be a strongly consistent assessment. Then $(\sigma, \beta)$ is Bayesian consistent and $\beta(m)$ is absolutely continuous with respect to $\tau$ for every isolated point $m \in M$.

Proof. We already know that strong consistency implies Bayesian consistency.

Now, let $(\sigma, \beta)$ be a strongly consistent assessment with supporting sequence $\left(\sigma^{k}, \beta^{k}\right)_{k \in \mathbb{N}}$ and let $m \in M_{i s o}$. Then for a Borel set $T_{B}$ with $\tau\left(T_{B}\right)=0$ the Bayesian consistency of $\left(\sigma^{k}, \beta^{k}\right)$ implies that

$$
\beta^{k}(m)\left(T_{B}\right) \cdot P^{\sigma^{k}}(m)=\int_{\{m\}} \beta^{k}\left(m^{\prime}\right)\left(T_{B}\right) d P^{\sigma^{k}}=\int_{T_{B}} \sigma_{1}^{k}(t)(m) d \tau=0 .
$$

Because $\{m\}$ is an open subset of $M, \sigma_{1}^{k}(t)(m)>0$ for all $t$. Hence, $P^{\sigma^{k}}(m)=\int_{T} \sigma_{1}^{k}(t)(m) d \tau>0$ and

$$
\beta^{k}(m)\left(T_{B}\right)=\frac{\int_{T_{B}} \sigma_{1}^{k}(t)(m) d \tau}{P^{\sigma^{k}}(m)}=0 .
$$

Since this relation holds for any $k, \beta(m)\left(T_{B}\right)=0$. So $\beta(m)$ is absolutely continuous with respect to $\tau$.

The foregoing theorem describes a condition that is necessary for an assessment to be strongly consistent. In the next theorem we show that this condition is also sufficient.

Theorem 3.5.3 Let $\Gamma$ be a signaling game and let $(\sigma, \beta)$ be an assessment. If $(\sigma, \beta)$ is Bayesian consistent and $\beta(m)$ is absolutely continuous with respect to $\tau$ for every isolated point $m \in M$, then $(\sigma, \beta)$ is strongly consistent.

The proof of this theorem will be based on three lemmas. First we need some notation.

Let $M^{*}=\left\{m_{1}, m_{2}, \ldots\right\}$ be a countable dense subset of $M$ and for every $m \in M_{i s o}$ let $b(m): T \rightarrow \mathbb{R}$ be a density function of $\beta(m)$ with respect to $\tau$ (i.e. $\beta(m)\left(T_{B}\right)=\int_{T_{B}} b(m)(t) d \tau$ for all $T_{B}$ ). Note that Radon-Nikodym's theorem guarantuees the existence of this density function.

For $k \in \mathbb{N}$ we define the mapping $\hat{\sigma}_{1}^{k}: T \rightarrow \mathcal{M}(M)$ by

$\hat{\sigma}_{1}^{k}(t)\left(M_{B}\right):=\sigma_{1}(t)\left(M_{B}\right)+\frac{1}{k^{2}}\left[\sum_{\substack{i \geq k \\ m_{i} \in M_{B} \cap M_{a c c u}}} \frac{1}{i^{2}}+\sum_{m_{i} \in M_{B} \cap M_{i a o}} \frac{1}{i^{2}}\left[b\left(m_{i}\right)(t) \vee k+\frac{1}{k}\right]\right]$. 
Then $\lim _{k \rightarrow \infty} \hat{\sigma}_{1}^{k}(t)(M)=1$, because for all $k$

$$
1 \leq \hat{\sigma}_{1}^{k}(t)(M) \leq 1+\frac{1}{k^{2}} \sum_{m_{i}} \frac{1}{i^{2}}\left[k+\frac{1}{k}\right]=1+\left[\frac{1}{k}+\frac{1}{k^{3}}\right] \sum_{m_{i}} \frac{1}{i^{2}} .
$$

Note that $\sum_{m_{4}} \frac{1}{i^{2}}$ is finite since $M^{*}$ is countable. Let, for very $k \in \mathbb{N}$ and $t \in T, R^{k}(t):=1 / \hat{\sigma}_{1}^{k}(t)(M)$. Obviously, $0<R^{k} \leq 1$ is measurable on $T$ for all $k$ and $\lim _{k \rightarrow \infty} R^{k}(t)=1$ for any $t$.

We consider the behavior strategy $\sigma_{1}^{k}: T \rightarrow \mathcal{P}(M)$ with

$$
\sigma_{1}^{k}(t)\left(M_{B}\right):=R^{k}(t) \cdot \hat{\sigma}_{1}^{k}(t)\left(M_{B}\right)
$$

for all $t$ and $M_{B}$. By Lemma 3.5.1, the set

$$
M^{*}(k):=\left\{m_{i} \in M_{\text {ccoul }} \mid i \geq k\right\} \cup M_{\text {iso }} \subset M^{*}
$$

is dense in $M$. So, if $M_{B}$ is an open set, $M^{*}(k) \cap M_{B} \neq \phi$. Hence, $\sigma_{1}^{k}(t)\left(M_{B}\right)>0$ for every $k$ and $t$ which implies that the probability measure $\sigma_{1}^{k}(\dot{t})$ is strictly positive.

Lemma 3.5.4 For every $t$, the sequence $\left(\sigma_{1}^{k}(t)\right)_{k \in \mathbb{N}}$ converges pointwise to $\sigma_{1}(t)$.

The proof of this result is straightforward.

For $k \in \mathbb{N}$ and Borel set $T_{B}$, we introduce

$$
\beta^{k}(m)\left(T_{B}\right):= \begin{cases}\frac{\int_{T_{B}} \sigma_{1}^{k}(t)(m) d \tau}{\int_{T} \sigma_{1}^{k}(t)(m) d \tau} & \text { if } m \in M^{*}(k) \\ \frac{\int_{T_{B}} R^{k}(t) d \beta(m)}{\int_{T} R^{k}(t) d \beta(m)} & \text { if } m \notin M^{*}(k) .\end{cases}
$$

Note that the two denominators in this definition are nonzero. If, for instance, $f_{T} \sigma_{1}^{k}(t)(m) d \tau=0$ for an $m \in M^{*}(k)$, then $\sigma_{1}^{k}(t)(m)=0, \tau$-allmost everywhere. However, by construction, $\sigma_{1}^{k}(t)(m)>0$ for all $t$.

Lemma 3.5.5 For every $m$, the sequence $\left(\beta^{k}(m)\right)_{k \in \mathbb{N}}$ converges pointwise to $\beta(m)$. 
Proof. (a) If $m \notin M_{i s a}$, then $m \notin M^{*}(k)$ for large $k$. Then the dominated convergence theorem implies that for all $T_{B}$ it holds that $\lim _{k \rightarrow \infty} \beta^{k}(m)\left(T_{B}\right)=$ $\frac{\int_{T_{B}} d \beta(m)}{\int_{T} d \beta(m)}=\beta(m)\left(T_{B}\right)$.

(b) Let $m \in M_{\text {iso }}$ and let $T_{B}$ be fixed. Then $\{m\}$ is a Borel set and

$$
\begin{aligned}
\int_{T_{B}} \sigma_{1}^{k}(t)(m) d \tau & =\int_{T_{B}} R^{k}(t) \cdot \hat{\sigma}_{1}^{k}(t)(m) d \tau \\
& =\int_{T_{B}} R^{k}(t) \cdot \sigma_{1}(t)(m) d \tau+\frac{1}{k^{2}} \frac{1}{i^{2}} \int_{T_{B}} R^{k}(t)\left[b(m)(t) \vee k+\frac{1}{k}\right] d \tau .
\end{aligned}
$$

Next we distinguish two cases.

(b1) Suppose that $\int_{T} \sigma_{1}(t)(m) d \tau>0$.

By the dominated convergence theorem

$$
\lim _{k \rightarrow \infty} \int_{T_{B}} R^{k}(t) \sigma_{1}(t)(m) d \tau=\int_{T_{B}} \sigma_{1}(t)(m) d \tau \text { for all } T_{B}
$$

Hence, (3.1) in combination with (3.2) implies that

$\lim _{k \rightarrow \infty} \beta^{k}(m)\left(T_{B}\right)=\frac{\int_{T_{B}} \sigma_{1}(t)(m) d \tau}{\int_{T} \sigma_{1}(t)(m) d \tau}$.

The Bayesian consistency of $(\sigma, \beta)$ implies that

$$
\int_{T_{B}} \sigma_{1}(t)(m) d \tau=\int_{\{m\}} \beta\left(m^{\prime}\right)\left(T_{B}\right) d P^{\alpha}=\beta(m)\left(T_{B}\right) \int_{T} \sigma_{1}(t)(m) d \tau .
$$

So

$$
\beta(m)\left(T_{B}\right)=\frac{\int_{T_{B}} \sigma_{1}(t)(m) d \tau}{\int_{T} \sigma_{1}(t)(m) d \tau}=\lim _{k \rightarrow \infty} \beta^{k}(m)\left(T_{B}\right) .
$$

(b2) Suppose that $\int_{T} \sigma_{1}(t)(m) d \tau=0$.

With (3.1) and (3.2) it follows that

$$
\beta^{k}(m)\left(T_{B}\right)=\frac{\int_{T_{B}} R^{k}(t)\left[b(m)(t) \vee k+\frac{1}{k}\right] d \tau}{\int_{T} R^{k}(t)\left[b(m)(t) \vee k+\frac{1}{k}\right] d \tau}
$$

Together with the dominated convergence theorem this leads to

$$
\lim _{k \rightarrow \infty} \beta^{k}(m)\left(T_{B}\right)=\frac{\int_{T_{B}} b(m)(t) d \tau}{\int_{T} b(m)(t) d \tau}=\beta(m)\left(T_{B}\right) .
$$


This completes the proof.

Lemma 3.5.6 For any $k,\left(\sigma^{k}, \beta^{k}\right)$ is Bayesian consistent.

Proof. In this proof $k \in \mathbb{N}$ is fixed.

(a) For all $T_{B}$ and any Borel set $M_{B} \subset M^{*}(k)$

$$
\begin{aligned}
\int_{M_{B}} \beta^{k}(m)\left(T_{B}\right) d P^{\sigma^{k}} & =\sum_{m \in M_{B}} \beta^{k}(m)\left(T_{B}\right) \cdot P^{\sigma^{k}}(m) \\
& =\sum_{m \in M_{B}} \frac{\int_{T_{B}} \sigma_{1}^{k}(t)(m) d \tau}{P^{k}(m)} P^{\sigma^{k}}(m) \\
& =\sum_{m \in M_{B}} \int_{T_{B}} \sigma_{1}^{k}(t)(m) d \tau \\
& =\int_{T_{B}} \sigma_{1}^{k}(t)\left(M_{B}\right) d \tau
\end{aligned}
$$

where the fourth equality is a consequence of the dominated convergence theorern.

(b) In this part of the proof we restrict ourselves to the set $M^{\prime}:=M \backslash M^{*}(k)$. First we introduce for every Borel subset $M_{B}^{\prime}$ of $M^{\prime \prime}$ the measure $\kappa\left(M_{B}^{\prime}\right)$ on $T$ as follows: for a Borel set $T_{B}^{4}, \kappa\left(M_{B}^{\prime}\right)\left(T_{B}\right):=\int_{T_{B}} \sigma_{1}(t)\left(M_{B}^{\prime}\right) d \tau$.

The Bayesian consistency of $(\sigma, \beta)$ implies that $\kappa\left(M_{B}^{\prime}\right)\left(T_{B}\right)=\int_{M_{B}^{\prime}} \beta(m)\left(T_{B}\right) d P^{\sigma}$.

With the help of Lemma 3.4 .2 this leads to

$$
\int_{T_{B}} R^{k}(t) d \kappa\left(M_{B}^{\prime}\right)=\int_{M_{B}^{\prime}}\left[\int_{T_{B}} R^{k}(t) d \beta(m)\right] d P^{\sigma}
$$

Then for every Borel set $M_{B}^{t}$

$$
\begin{aligned}
P^{\sigma^{k}}\left(M_{B}^{t}\right) & =\int_{T} R^{k}(t) \cdot \hat{\sigma}_{1}^{k}(t)\left(M_{B}^{\prime}\right) d \tau=\int_{T} R^{k}(t) \cdot \sigma_{1}(t)\left(M_{B}^{\prime}\right) d \tau \\
& =\int_{T} R^{k}(t) d \kappa\left(M_{B}^{t}\right)=\int_{M_{B}^{\prime}}\left[\int_{T} R^{k}(t) d \beta(m)\right] d P^{\sigma} .
\end{aligned}
$$

So we may conclude that $m \rightarrow \int_{T} R^{k}(t) d \beta(m)$ is a density function of $p^{\sigma^{k}}$ with respect to $P^{o}$ on $M^{\prime}$. Hence, for all $T_{B}$

$$
\begin{aligned}
\int_{M_{s}^{*}} \beta^{k}(m)\left(T_{B}\right) d P^{\sigma^{k}} & =\int_{M_{B}^{*}} \beta^{k}(m)\left(T_{B}\right)\left[\int_{T} R^{k}(t) d \beta(m)\right] d P^{\sigma} \\
& =\int_{M_{B}^{*}} \frac{\int_{T_{B}} R^{k}(t) d \beta(m)}{\int_{T} R^{k}(t) d \beta(m)}\left[\int_{T} R^{k}(t) d \beta(m)\right] d P^{\sigma}
\end{aligned}
$$




$$
\begin{aligned}
& =\int_{M_{B}^{\prime}}\left[\int_{T_{B}} R^{k}(t) d \beta(m)\right] d P^{\sigma}=\int_{T_{B}} R^{k}(t) d \kappa\left(M_{B}^{\prime}\right) \\
& =\int_{T_{B}} R^{k}(t) \cdot \sigma_{1}(t)\left(M_{B}^{\prime}\right) d \tau=\int_{T_{B}} \sigma_{1}^{k}(t)\left(M_{B}^{\prime}\right) d \tau .
\end{aligned}
$$

(c) The parts (a) and (b) imply that for all $T_{B}$ and $M_{B}$

$$
\begin{aligned}
\int_{M_{B}} \beta^{k}(m)\left(T_{B}\right) d P^{\sigma^{k}} & =\int_{M_{B} \cap M^{*}(k)} \beta^{k}(m)\left(T_{B}\right) d P^{\sigma^{k}}+\int_{M_{B} \backslash M^{*}(k)} \beta^{k}(m)\left(T_{B}\right) d P^{\sigma^{k}} \\
& =\int_{T_{B}} \sigma_{1}^{k}(t)\left(M_{B} \cap M^{*}(k)\right) d \tau+\int_{T_{B}} \sigma_{1}^{k}(t)\left(M_{B} \backslash M^{*}(k)\right) d \tau \\
& =\int_{T_{B}} \sigma_{1}^{k}(t)\left(M_{B}\right) d \tau .
\end{aligned}
$$

This completes the proof of the theorem.

Corollary 3.5.7 Let $\Gamma$ be a signaling game and let $(\sigma, \beta)$ be an assessment. Then $(\sigma, \beta)$ is strongly consistent if and only if $(\sigma, \beta)$ is Bayesian consistent and $\beta(m)$ is absolutely continuous with respect to $\tau$ for every isolated point $m \in M$.

Using this characterization, we can investigate the consequences of strong consistency in some special classes of signaling games.

Corollary 3.5.8 If the message space of a signaling game contains no isolated points or the type space is discrete, strong consistency is equivalent to Bayesian consistency.

In particular, this holds for finite and discrete signaling games.

Corollary 3.5.9 If the message space of a signaling game is discrete and the type space is not, then an assessment is strongly consistent if and only if it is Bayesian consistent and $\beta(m)$ is absolutely continuous w.r.t. $\tau$ for every message $m$.

\subsection{Other consistency concepts}

In the following theorem, we show that every assessment which is consistent w.r.t. some appropriate definition $\varphi$ automatically satisfies the conditions of the characterization in the previous section and is therefore strongly consistent. So, every appropriate consistency concept is a refinement of strong consistency. 
Theorem 3.6.1 Let $\varphi$ be an appropriate consistency concept. If an assessment $(\sigma, \beta)$ is consistent w.r.t. $\varphi$ then $(\sigma, \beta)$ is Bayesian consistent and $\beta(m)$ is absolutely continuous w.r.t. $\tau$ for every isolated point $m$.

Proof. Let $\varphi$ be an appropriate consistency concept and $(\sigma, \beta)$ a consistent assessment w.r.t. $\varphi$ with supporting sequence $\left(\sigma^{k}, \beta^{k}\right)_{k \in \text { N }}$. Since $\varphi$ is appropriate, $(\sigma, \beta)$ must be Bayesian consistent.

Now, take an arbitrary isolated point $m \in M$. Then, $\{m\}$ is open and therefore $T \times\{m\}$ is an open subset of $T \times M$. By condition $B$,

$$
P^{\sigma^{k}}(m)=\int_{T} \sigma_{1}^{k}(t)(m) d \tau>0
$$

for every $k$. Since $\left(\sigma^{k}, \beta^{k}\right)$ is Bayesian consistent, it follows that

$$
\beta^{k}(m)\left(T_{B}\right) \cdot P^{\sigma^{k}}(m)=\int_{\{m\}} \beta^{k}\left(m^{k}\right) d P^{\sigma^{k}}=\int_{T_{B}} \sigma_{1}^{k}(t)(m) d \tau
$$

for every $T_{B}$, which means that

$$
\beta^{k}(m)\left(T_{B}\right)=\frac{\int_{T_{B}} \sigma_{1}^{k}(t)(m) d \tau}{P^{\sigma^{k}}(m)}
$$

for every $T_{B}$. If $\tau\left(T_{B}\right)=0$, we have $\beta^{k}(m)\left(T_{B}\right)=0$ for every $k$. Since $m$ is in every dense subset of $M$, condition $C$ implies that $\beta^{k}(m)$ converges pointwise to $\beta(m)$, which implies that $\beta(m)\left(T_{B}\right)=\lim _{k \rightarrow \infty} \beta^{k}(m)\left(T_{B}\right)=0$. Hence, $\beta(m)$ is absolutely continuous w.r.t. $\tau$.

In view of Corollary 3.5.7, we arrive at the following conclusion.

Theorem 3.6.2 Every appropriate consistency concept is a refinement of strong consistency.

In the following example we consider a signaling game in which strong consistency excludes some sequential equilibria. By Theorem 3.6.2 it follows that every appropriate consistency concept excludes these equilibria.

Example 3.2. Let $\Gamma$ be a signaling game in which $T=[0,1], M=$ $\{y, n\}, A=\{b, c\}$ and $T$ is the uniform distribution on $T$. The payoffs are
given by

$$
\begin{aligned}
& u_{1}\left(t, m_{1}, a\right):=0 \quad \text { for all } t, m, a \\
& u_{2}(t, m, a):= \begin{cases}t & \text { if } a=b \\
0 & \text { if } a=c .\end{cases}
\end{aligned}
$$


We define the assessment $(\sigma, \beta)$ by

$$
\begin{aligned}
\sigma_{1}(t): & = \begin{cases}\delta_{y} & \text { if } t=0 \\
\delta_{n} & \text { if } t>0\end{cases} \\
\sigma_{2}(y): & =\delta_{c} \\
\sigma_{2}(n): & =\delta_{b} \\
\beta(y): & =\delta_{0} \\
\beta(n): & =\tau .
\end{aligned}
$$

It can be shown that $(\sigma, \beta)$ is a sequential equilibrium. However, $(\sigma, \beta)$ is not strongly consistent since $\beta(y)$ is not absolutely continuous w.x.t. $\tau$.

\subsection{Structurally consistent assessments}

Finally, we consider a different form of consistency as introduced by Kreps and Wilson (1982). They call an assessment structurally consistent if for every information set there is a behavior strategy profile such that this information set will be reached with positive probability and the beliefs at this information set are completely determined by Bayes' rule. If we formulate this concept for signaling games, we obtain the following definition.

An assessment $(\sigma, \beta)$ is called structurally consistent if for every $m \in M$ there is a BSP $\bar{\sigma}$ such that $P^{\bar{\sigma}}(m)>0$ and for every $T_{B}$

$$
\beta(m)\left(T_{B}\right)=\frac{\int_{T_{B}} \bar{\sigma}_{1}(t)(m) d \tau}{P^{\bar{\sigma}}(m)} .
$$

In the following theorem we characterize the class of structurally consistent assessments in signaling games with at least two messages.

Theorem 3.7.1 For a signaling game with at least two different messages an assessment $\left(\sigma_{n} \beta\right)$ is structurally consistent if and only if, for every $m$, there is a constant $c_{m}>0$ such that for all $T_{B}$

$$
\beta(m)\left(T_{B}\right) \leq c_{m} \cdot \tau\left(T_{B}\right) .
$$

Proof. " $\Rightarrow$ " Let $(\sigma, \beta)$ be a structurally consistent assessment and $m$ a message in $M$. By definition, there is a BSP $\tilde{\sigma}$ such that $P^{\sigma}(m)>0$ and for any $T_{B}$

$$
\beta(m)\left(T_{B}\right)=\frac{\int_{T_{B}} \bar{\sigma}_{1}(t)(m) d \tau}{P^{\tilde{\sigma}}(m)} \leq \frac{1}{P^{\tilde{\sigma}}(m)} \tau\left(T_{B}\right) .
$$


Now choose $c_{m}:=\frac{1}{P^{\ddot{\sigma}}(m)}$.

$" \Leftarrow$ Suppose, for every $m$ there is a constant $c_{m}>0$ such that for all $T_{B}$ we have $\beta(m)\left(T_{B}\right) \leq c_{m} \cdot \tau\left(T_{B}\right)$. Then, obviously, $\beta(m)$ is absolutely continuous w.r.t. $\tau$ for every $m$.

Let $m \in M$ and let $b(m): T \rightarrow \mathbb{R}$ be a density function of $\beta(m)$ with respect to $T$. Then, for any $T_{B}$

$$
\int_{T_{B}} b\left(m_{i}\right)(t) d \tau=\beta(m)\left(T_{B}\right) \leq c_{m} \cdot \tau\left(T_{B}\right)=\int_{T_{B}} c_{m} d \tau
$$

Hence, there is a Borel set $T_{B}^{*}$ with $\tau\left(T_{B}^{*}\right)=1$ such that $b(m)(t) \leq c_{m}$ for all $t \in T_{B}^{*}$. In order to define a behavior strategy $\bar{\sigma}_{1}$ for player 1 , we take a message $\hat{m} \neq m$ in $M$. If $t \notin T_{B}^{*}$, let $\bar{\sigma}_{1}(t)$ be an arbitrary probability measure on $M$. Otherwise, for a Borel set $M_{B}$

$$
\vec{\sigma}_{1}(t)\left(M_{B}\right):= \begin{cases}\frac{1}{b(m)(t)} & \text { if } m \in M_{B} \text { and } \tilde{m} \in M_{B} \\ 1-\frac{b(m)(t)}{c_{m}} & \text { if } m \in M_{B} \text { and } \dot{m} \notin M_{B} \\ 0 & \text { otherwise. }\end{cases}
$$

Then, $P^{\tilde{\sigma}}(m)=\int_{T_{\dot{B}}^{*}} \bar{\sigma}_{1}(t)(m) d \tau=\int_{T_{\dot{B}}^{*}} \frac{b(m)(t)}{c_{m}} d \tau=\int_{T} \frac{b(m)(t)}{c_{m}} d \tau$ $=\frac{1}{c_{m}} \beta(m)(T)=\frac{1}{c_{m}}>0$. Furthermore, for any $T_{B}$,

$$
\begin{aligned}
\frac{\int_{T_{m}} \tilde{\sigma}_{1}(t)(m) d \tau}{P^{\tilde{\sigma}}(m)} & =\frac{\int_{T_{B} \cap T_{B}} \bar{\sigma}_{1}(t)(m) d \tau}{P^{\sigma}(m)}=\frac{\frac{1}{\sigma_{m}} \int_{B_{B} T_{B}} b(m)(t) d \tau}{\frac{1}{c_{m}}} \\
& =\int_{T_{B}} b(m)(t) d \tau=\beta(m)\left(T_{B}\right) .
\end{aligned}
$$

So $(\sigma, \beta)$ is structurally consistent.

From the theorem above, it follows that every structurally consistent assessment $(\sigma, \beta)$ must have the property that the local belief $\beta(m)$ is absolutely continuous w.r.t. $\tau$ for every $m$.

Consider, for instance, an infinite signaling game with $T=M=[0,1], \tau$ equal to the uniform ditribution on $T$ and an assessment $(\sigma, \beta)$ in which $\beta(0)=\delta_{0}$. Such an assessment is ruled out by structural consistency since
$\beta(0)$ is not absolutely continuous w.r.t. $\tau$. 


\section{Part II}

\section{Buying information}




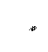




\section{Chapter 4}

\section{Buying information in extensive form games}

\subsection{Introduction}

This chapter is based on Perea y Monsuwé (1997a).

Many economic situations are characterized by uncertainty. However, often the decision makers have the possibility to obtain additional information about uncertain elements before making decisions. Additional information can be acquired, for instance, through financial newsletters, laboratory tests, government statistical reports or consumer marketing surveys. The key question which arises is: Which information should be chosen by the decion maker and how much should he pay for this?

There is a huge literature dealing with the value of information in economic situations under uncertainty. Without even trying to be complete, we discuss some papers that are related to the approach to be developed in this chapter. Sakai (1985) considers a duopoly model in which two firms produce the same good with linear cost functions. However, the marginal costs are not known to both firms. Before choosing a price and quantity, the firms have the possibility to consult a market research agency which reveals to a firm either its own marginal cost or the marginal cost of the opponent or both. In this chapter, it is investigated how the best decision functions of the firms and their expected payoffs depend on the possible combinations of information chosen by the firms.

Admati and Pfleiderer (1986) analyze a situation where traders on a speculative market purchase private information about the payoff of a risky asset from a monopolistic information seller.

In Allen (1986), a model is investigated in which traders sell information 
about the current state of the world to each other.

A common feature in all of the above mentioned articles is the fact that information is treated as a good which helps the decision makers to maximize state dependent utilities under uncertainty. Since the context in which the information transaction takes place differs in every paper, each of them has its own interpretation of the value of information. Roughly speaking, the value of information reflects the maximum amount of money that a decision maker, who initially has no information, would be willing to pay to acquire the information. However, this maximum amount of money depends on many facts, such as the way in which the situation is modeled and the behavior of ather decision makers. Therefore, the definition of the value of information should be connected explicitly to the model which is used.

Also in the garme theoretic literature, the problem of the value of information has been discussed. For instance, Kamien et al. (1990) consider the situation where a so-called 'maven' can transmit information to the players of an extensive form game in which the maven is not a participant. The 'inducible set' is defined to be the set of all outcomes which can be induced by unique Nash equilibria of a game resulting from the maven's transmission of information. In words, the inducible set reflects the maven's ability to manipulate the game. The maximum payoff that the maven can guarantee for himself is called the value of the maven's power.

In Levine and Ponssard (1977), a move of nature selects the payoffs of a non-cooperative game before the actual game starts. The players are only partially informed about the outcome of the move of nature. The paper discusses how different types of information structures, such as secret information (one player acquires information but other players are ignorant of this fact), private information (one player acquires information and other players know it) and public information (all players acquire information and everybody knows this) influence the value of information.

For further reading on the value of information, we refer to Milne and Shefrin (1987), Green (1981), Neyman (1991) and Gilboa and Lehrer (1991).

In nearly all papers in this field (an exception being the paper by Kamien et al. (1990)) the person or organization who transmits the information does not play a strategic role in the madel: he is just there to provide the decision makers with additional information but has no strategic motivation to do so. The aim of this chapter is to develop a general game theoretic model in which decision makers can buy information from a seller who, himself, has strategic incentives. Formally, we model this situation by assuming that the players of an extensive form game, which we call the base game, have the possibility to buy information from a monopolistic information seller who, at the beginning of the game, chooses a price for every information that 
is offered. In this way, we obtain a new game, called the extended game, which is played by the information seller and the players of the base game, called information buyers. We define the information value to be the highest payoff that the information seller can obtain in a sequential equilibrium of the extended game. Intuitively, it reflects the highest amount of money that the seller can possibly extract from the buyers, assuming that seller and buyers act to the standards of a sequential equilibrium.

Technically speaking, the process of buying information is performed as follows. At every information set of the base game, the seller offers a collection of partitions which divide the information set into disjoint subsets of nodes. If the corresponding buyer purchases a certain partition, the seller will reveal which subset of the partition has been reached. Until section 8 , we assume that the other buyers do not observe which partition has been bought.

At the beginning of the extended game, the seller chooses a price for every partition that is offered. We assume that the trivial partition (giving no additional information) is offered at every information set and should always be sold at price zero. Therefore, buyers always have the possiblity to refrain from purchasing information by buying the trivial partition at price zero. The price vector, containing all prices, is observed by all buyers before the actual game starts.

The expected payoff for the seller consists of the expected amount obtained by selling information to the buyers whereas the expected payoff for a buyer is the difference between the expected payoff at the terminal nodes of the base game and the expected amount to be paid for buying information.

Obviously, the extended game is not a finite extensive form game, since the seller can choose from a continuum of prices. The existence of sequential equilibria in the extended game is therefore not straightforward. However, we show that every extended game has at least one sequential equilibrium. Moreover, it turns out that the payoff for the seller reaches a maximum on the set of sequential equilibria. It is because of this fact that the information value, being the highest possible payoff for the seller in a sequential equilibrium, is well defined.

The computation of the information value, however, might cause serious problems since the extended game is in general very large. First of all, it consists of infinitely many subgames: after every possible price vector, a subgame, consisting of a finite extensive form game, starts. Moreover, every subgame can be very large because in general it contains many information sets and each information set might contain a huge amount of nodes, especially if many partitions are offered. The definition of behavior strategies and belief systems requires therefore a huge complexity.

We present a way to overcome these difficulties by developing an efficient 
method to compute the information value. The method consists of two steps. In the first step, it is shown that in every subgame, behavior strategies and belief systems can be replaced by much more compact reduced behavior strategies and reduced belief systems, which have the same size as those of the base game. Secondly, we prove that the information value is equal to the highest payoff that the seller can obtain in any reduced sequential equilibrium (i.e. sequential equilibrium in reduced behavior strategies and reduced belief systems) of any subgame.

Next, we discuss some intuitive properties of the information value. Consider, for instance, a situation in which the seller decides to enlarge his set of partitions. It turns out that this will lead to an increase in the information value. Intuitively, this is clear since expanding the set of partitions makes the seller more powerful. However, in Example 4.3 it is shown that replacing a partition by a more informative one can lead to a decrease in the information value because more informative partitions might hurt the seller. This is a result which is less intuitive.

Moreover, every optimal sequential equilibrium (i.e. sequential equilibrium supporting the information value) has the property that a partition should always be sold at a price which makes the buyer indifferent between buying this partition and buying the trivial one, given that the corresponding information set is reached with positive probability and the partition is bought with positive probability. The intuition behind this is the fact that a higher price would make buying no information more attractive than buying the pertition and a lower price would give the seller room to improve his payoff by raising his price a little bit.

By providing some examples, we illustrate a few less intuitive phenomena which might occur. The first example shows that in some cases it is optimal for the seller that a buyer strictly randomizes over buying different partitions. In the second example, the information value can only be supported by sequential equilibria in which player 2 refuses to buy information. Both phenomena are due to the fact that purchasing information by a certain buyer can force other buyers to act in a way which is bad for the seller. In the standard model, we assume that the transaction of information is private, meaning that buyers do not observe which partitions have been bought by the others. It is an interesting question what would happen if the information transactions would be made public. Two examples show that publicly observed information transactions can either increase or decrease the information walue.

Until this point, we assumed that the base game is a single appearance game, meaning that in every play of the game each player appears at most once. In the final section, we investigate the possibilities and problems which arise if 
we want to extend the model to base games which are not single appearance games. The main conclusion is that this extension is possible, but many additional technical problems arise due to perfect recall.

The outline of the chapter is as follows. In section 2 , we decribe how the process of buying information is formally implemented. In section 3 , we prove the existence of sequential equilibria in the extended game and give the definition of the information value. A method for the computation of the information value is given in section 4 , whereas section 5 discusses some intuitive properties of the information value. Some examples in section 6 illustrate typical phenomena which can be encountered in extended games. Section 7 considers the situation in which information transactions are publicly observed whereas section 8 discusses the extension of the model to base games which are not single appearance games.

\title{
4.2 Implementation of buying information
}

\author{
Rules of the extended game
}

We extend the model of an extensive form game to a situation in which every player is able to buy 'information' at each of his information sets. By 'information' we mean a. mechanism which tells the player that the play of the game has reached a certain subset of the information set. Therefore, information can be identified with a partition which divides the information set into disjoint subsets of nodes. We assume that the information is offered by a so-called "information seller", who chooses a price for every partition at the beginning of the game. The game obtained in this way is called the extended game.

Formally, the extended game is played as follows. We start with an extensive form game $\Gamma$ which has perfect recall and contains $n$ players. This game is called the base game. For reasons to become clear in section 9 , we assume that $\Gamma$ is a singie appearance game, which means that every player appears at most once on every path of the game tree.

The extended game is a game with $n+1$ players, in which player $n+1$ is called the 'information seller' and players 1 to $n$ are 'information buyers'.

At every information set $h$, the seller offers a collection $\mathcal{P}_{h}$ of partitions on $h$. Each partition $P \in \mathcal{P}_{h}$ divides the set $h$ into disjoint subsets $X \in P$. We assume that the trivial partition (i.e. the partition revealing no additional information) is offered at every information set. 
At the beginning of the game, the seller chooses a price $c_{h}(P)$ for every information set $h$ and every partition $P \in \mathcal{P}_{h h}$. We assume that trivial partitions must be sold at price zero. Therefore, buying the trivial partition can be regarded as not buying any information at all. The prices $c_{h}(P)$ are collected in the price vector $c$.

The buyers observe the price vector $c$ and start playing the subgame $\Gamma(c)$ defined by the following rules. At every information set $h$, the corresponding buyer first chooses a partition $P \in \mathcal{P}_{h}$ and pays the price $c_{h}(P)$ to the seller. The actual partition chosen by the buyer is nat observed by the other buyers. The seller reveals to the buyer which subset $X \in P$ has been reached. If the buyer observes that the game is at subset $X$, he chooses a corresponding action $a_{X} \in A(h)$, where $A(h)$ is the set of actions available at $h$. We model this situation by assuming that the buyer chooses a vector $\left(a_{X}\right)_{X \in P}$ of actions.

At the end of the game, the expected payoff for the seller consists of the expected income of selling information to the buyers. The expected payoff for a buyer is the difference between the expected payoff at the terminal nodes of the base game and the expected amount paid for buying information.

The extended game as a formal extensive form game

The extended game can be described formally by the following extensive form game. The game starts at the root, where the seller chooses a price vector c. Every possible price vector $c$ leads to a subgame $\Gamma(c)$ in which the game tree is not dependent on $c$ but the payoffs at the terminal nodes are.

Consider, for example, the following fragment of a base game. 


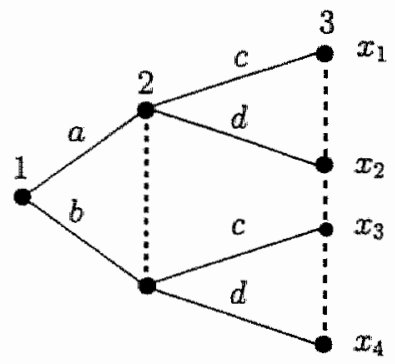

Figure 4.1

Suppose that the seller only offers trivial and perfect partitions. In every subgame $\Gamma(c)$, the corresponding fragment of the extended game would look as in figure 4.2 .

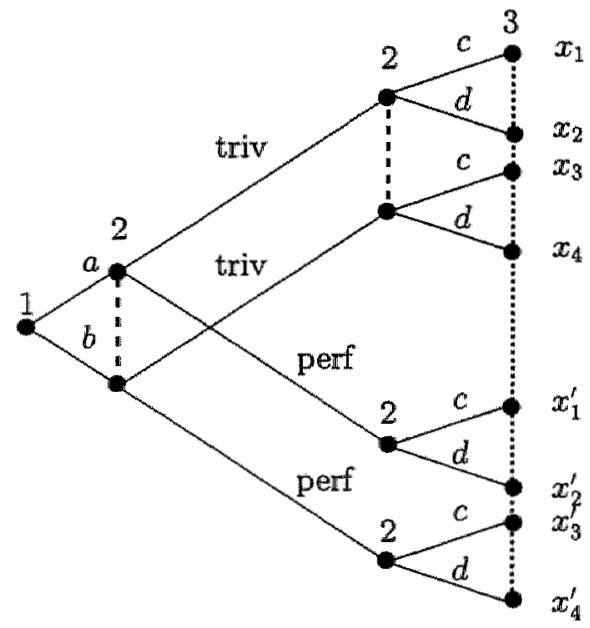

Figure 4.2

Note that the information set of player 3 has been duplicated since he does not know whether player 2 has bought the trivial or the perfect partition. However, the extensive form structures following the nodes $x_{i}$ and $x_{i}^{\prime}$ are identical. Moreover, player 2 does not appear in the continuation of the 
game which follows the information set of player 3 since we assume that the base game is a single appearance game. Since the games following the nodes $x_{i}$ and $x_{i}^{\prime}$ have the same payoffs for all players except player $2, x_{i}$ and $x_{i}^{\prime}$ can be regarded as identical nodes in the continuation of the game.

\subsection{Information value}

In this section, we define the information value of an extended game. To this purpose, we must specify what we mean by strategies, beliefs and sequential equilibria in an extended game.

\section{Sequential equilibria in extended game}

In an extended game, a behavior strategy for the seller is a probability measure $\mu$ on the set of all possible price vectors $c=\left(c_{h}(P)\right)_{P \in \mathcal{P}_{h}, h \in H}$.

For every price vector $c, \Gamma(c)$ denotes the subgame of the extended game which starts if $c$ is chosen. A behavior strategy profile for the buyers is a function $\sigma$ which assigns to every possible price vector $c$ a behavior strategy profile (BSP) $\sigma(c)$ in the subgame $\Gamma(c)$. Since $\Gamma(c)$ is a finite extensive form game, it is clear what we mean by a BSP in $\Gamma(c)$.

A belief system is a function $\beta$ assigning to every price vector $c$ a belief system $\beta(c)$ in the subgame $\Gamma(c)$.

For every subgame $\Gamma(c)$, consistency and sequential rationality of the assessment $(\sigma(c), \beta(c))$ is defined in the usual way (cf. Kreps and Wilson, 1982). The assessment $(\mu, \sigma, \beta)$ is called a sequential equilibrium if $(\sigma(c), \beta(c))$ is consistent and sequentially rational for every $c$ and the seller maximizes his expected payoff given $\sigma$.

Since the price vector is observed by all buyers, we may assume w.l.o.g. that the seller puts all weight on a single price vector.

Optimal sequential equilibria and information value

The extended game is not a finite extensive form game since the seller can choose from a continuum of price vectors. Therefore, the existence of a sequential equilibria is not obvious. However, the following theorem guarantees that every extended game has at least one sequential equilibrium. Moreover, 
we show that there is a sequential equilibrium which yields the maximal payoff for the seller on the set of all sequential equilibria. Such a sequential equilibrium is called optimal.

Theorem 4.3.1 (a) Every extended game has at least one sequential equilibrium.

(b) The payoff for the seller reaches a maximum on the set of sequential equilibria.

Proof. The outline of the proof is as follows. First, we show that there is a subgame $\Gamma\left(c^{*}\right)$ and a sequential equilibrium in $\Gamma\left(c^{*}\right)$ which gives the seller the highest payoff that can be obtained in any sequential equilibrium of any subgame. Afterwards, we construct a sequential equilibrium of the whole extended game in which the seller chooses the price vector $c^{*}$ with probability one. Finally, we show that this sequential equilibrium is optimal.

Let $S E(c)$ be the set of sequential equilibria $(\sigma(c), \beta(c))$ of the subgame $\Gamma(c)$ and let $S E^{*}$ be the union of the sets $S E(c)$. By $u_{n+1}(\sigma(c), \beta(c))$, we denote the expected payoff for the seller if $(\sigma(c), \beta(c))$ is played in the subgame $\Gamma$ (c). Let $\bar{c}$ be the difference between the highest and the lowest payoff at the terminal nodes of the base game $\Gamma$. Since no player is willing to pay more than $\bar{c}$ for any partition in a sequential equilibrium $(\sigma(c), \beta(c))$, it follows that $u_{n+1}$ can not be more than $|H| \cdot \bar{c}$ in any sequential equilibrium of $S E^{*}$, where $|H|$ is the number of information sets in $\Gamma$. Hence, the function $u_{n+1}$ is bounded on $S E^{*}$ and has therefore a supremum on $S E^{*}$.

Let $C$ be the set of price vectors $c$ with $c_{h}(P) \leq \bar{c}$ for every partition $P$ at every information set $h$. We have already seen that no player is prepared to pay more than $\bar{c}$ for any partition. Therefore, we may conclude that the supremum of $u_{n+1}$ on $S E^{*}$ is the same as the supremum on $S E^{*}(C)$, where $S E^{*}(C)$ is the the union of all sets $S E^{*}(c)$ with $c \in C$. Next, we show that the supremum of $u_{n+1}$ on $S E^{*}(C)$ is a maximum.

In order to show that the set $S E^{*}(C)$ is closed, consider a sequence $\left(\sigma^{k}\left(c^{k}\right), \beta^{k}\left(c^{k}\right)\right)$ of sequential equilibria in $S E^{*}\left(c^{k}\right)$ with $c^{k} \in C$, converging to an assessment $(\bar{\sigma}, \bar{\beta})$ of some subgame. W.l.o.g. we may assume that $c^{k}$ converges to some price vector $c \in C$. It can be seen easily that $(\bar{\sigma}, \bar{\beta})$ is a sequential equilibrium in $S E^{*}(c) \subset S E^{*}(C)$, which implies that $S E^{*}(C)$ is closed.

Hence, the set $S E^{*}(C)$ is closed and bounded and therefore compact. Furthermore, we know that $S E^{*}(C)$ is non-empty. Since the expected payoff $u_{n+1}$ depends continuously on the assessment $(\sigma(c), \beta(c))$ it follows that $u_{n+1}$ obtains a maximum on $S E^{*}(C)$. 
Therefore, there is a $c^{*} \in C$ and a sequential equilibrium $\left(\sigma^{*}\left(c^{*}\right), \beta^{*}\left(c^{*}\right)\right)$ in $S E^{*}\left(c^{*}\right)$ which gives the highest expected payoff to the seller among all assessments in $S E^{* *}$. We construct a sequential equilibrium $(\mu, \sigma, \beta)$ of the extended game in the following way.

Let $\mu:=\mathcal{b}_{c^{*}}$, which means that $\mu$ is the Dirac-measure on the point $c^{*}$. We define

$$
\sigma\left(c^{*}\right):=\sigma^{*}\left(c^{*}\right) \text { and } \beta\left(c^{*}\right):=\beta^{*}\left(c^{*}\right)
$$

whereas for $c \neq c^{*}, \sigma(c)$ and $\beta(c)$ are chosen in such a way that $(\sigma(c), \beta(c))$ is a sequential equilibrium in the subgame $\Gamma(c)$.

By construction, $(\mu, \sigma, \beta)$ is sequentially rational for the seller since he puts probability one on the vector $c^{*}$ which gives him the highest possible payoff that can be obtained in any sequential equilibrium of any subgame. Therefore, we may conclude that $(\mu, \sigma, \beta)$ is a sequential equilibrium in the extended game which gives the seller the highest possible payoff among the set of sequential equilibria of the extended game. So, $(\mu, \sigma, \beta)$ is optimal.

The payoff for the seller induced by an optimal sequential equilibrium is called the information value of the extended game. In other words, the information value is the highest amount that the seller can possibly extract from the buyers, given that seller and buyers play a sequentiall equilibrium.

\subsection{Computation of information value}

If we want to compute the information value of a given extended game, we are faced with two major problems. First of all, the definition of a sequential equilibrium requires that we specify strategies and beliefs for all possible subgames $\Gamma(c)$. Moreover, each of the subgames $\Gamma(c)$ can have a huge game tree, especially when the seller offers a lot of partitions. Consider, for instance, the example of Figures 1 and 2. Even in this simple case, where the seller only offers the trivial and perfect partition, the extended game contains four player 2 information sets, whereas the base game only contained one. In addition, the player 3 information set has grown from size four in the base game to size eight in the extended game, due to the fact that player 3 does not observe the partition bought by player 2. From this example, it can be seen easily that in the extreme case, in which the seller offers all possible partitions, the number of information sets and the size of the individual information sets grows exponentially with the size of the base game. Therefore, the definition of an assessment $(\sigma(c), \beta(c))$ for a given subgame $\Gamma(c)$ requires a complexity which can grow exponentially with the size of the base game. 
In this section, we present a method to overcome the two problems mentioned above and use it to develop a more efficient way to compute the information value. The method consists of two steps. In the first step we show that for a given subgame $\Gamma(c)$, the payoffs induced by sequential equilibria in $\Gamma(c)$ coincide with the payoffs induced by so-called reduced sequential equilibria of $\Gamma(c)$. Reduced sequential equilibria have the advantage that their definition requires the same complexity as the definition of sequential equilibria of the base game.

In step 2, we show that the information value is equal to highest payoff for the seller which can be obtained in any reduced sequential equilibrium of any subgame.

Therefore, the problem of computing the information value is reduced to the problem of finding a price vector $c$ and a corresponding reduced sequential equilibrium in $\Gamma(c)$ which gives maximal payoff to the seller.

\section{Reduced sequential equilibria of subgames}

Consider a fixed subgame $\Gamma(c)$ of the extended game. For every information set $h$ of the base game, a reduced local strategy for the corresponding buyer is vector $\left(P,\left(a_{X}\right)_{X \in P}\right)$ where $P$ is a partition offered at $h$ and $a_{X}$ is an action in $A(h)$. Intuitively, $a_{X}$ is the action that the player will choose if he buys the partition $P$ and the seller reveals that the play of the game has reached the subset $X$. The set of reduced local strategies at $h$ is denoted by $\bar{A}(h)$. A reduced behavior strategy profile is a vector $\bar{\sigma}$ assigning to every information set in the base game a randomization over the set of reduced local strategies. A reduced belief system $\bar{\beta}$ is simply a belief system of the base game.

A pair $(\bar{\sigma}, \bar{\beta})$ is called a reduced assessment.

We say that $(\bar{\sigma}, \bar{\beta})$ is sequentially rational if at each information set $h$ of the base game, the corresponding buyer maximizes his expected payoff, given his beliefs at $h$.

In the obvious way, $\bar{\sigma}$ induces a probability distribution on the nodes of the base game. The reduced assessment $(\bar{\sigma}, \bar{\beta})$ is said to be Bayesian consistent if at each information set $h$ of the base game which is reached with positive probability it holds that

$$
\bar{\beta}_{h}(x)=\frac{\mathbb{P}_{\tilde{\sigma}}(x)}{\mathbb{P}_{\tilde{\sigma}}(h)}
$$

for every $x \in h$. Here, $\mathbb{P}_{\bar{\sigma}}(x)$ and $\mathbb{P}_{\tilde{\sigma}}(h)$ denote the probabilities that $x$ and $h$ are reached respectively. 
We call $(\bar{\sigma}, \bar{\beta})$ consistent if it is the limit of some sequence $\left(\bar{\sigma}^{k}, \bar{\beta}^{k}\right)$ of completely mixed, Bayesian consistent assessments. By completely mixed, we mean that $\tilde{\sigma}^{k}$ puts positive probability on every reduced local strategy.

The reduced assessment $(\bar{\sigma}, \bar{\beta})$ is called a reduced sequential equilibrium in the subgame $\Gamma(c)$ if it is sequentially rational and consistent.

In the following lemma, we show that sequential equilibria and reduced sequential equilibria of a subgame induce the same payoffs.

Lemma 4.4.1 For every subgame $\Gamma(c)$, the payoffs induced by sequential equilibria in $\Gamma(c)$ coincide with the payoffs induced by reduced sequential equilibria in $\Gamma(c)$.

Proof. Let the subgame $\Gamma(c)$ be fixed and let $\left(\sigma_{3} \beta\right)$ be a sequential equilibrium in $\Gamma(c)$. We transform $(\sigma, \beta)$ into a reduced sequential equilibrium $\left(\bar{\sigma}_{3}, \bar{\beta}\right)$ with the same payoffs.

Note that $\sigma$ assigns to every information set $h$ of the base game and every partition $P$ at $h$ a probability $\sigma(P)$. Furthermore, it assigns to every partition $P$ at $h$ and every subset $X \in P$ a probability ditribution $\sigma_{P, X}$ on the set $A(h)$ of actions available at $h$.

For every reduced local strategy $\bar{a}=\left(P,\left(a_{X}\right)_{x \in P}\right)$ we define

$$
\bar{\sigma}(\vec{a}):=\sigma(P) \cdot \prod_{X \in P} \sigma_{P_{r} X}\left(a_{X}\right)
$$

where $\sigma(P)$ is the probability that $P$ is bought in $\sigma$ and $\sigma_{P, X}(a)$ is the probability that the action $a$ is chosen in $\sigma$ if the player has bought partition $P$ and subset $X \in P$ has been reached.

For every node $x \in h$, the belief $\vec{\beta}(x)$ is given by

$$
\bar{\rho}(x):=\sum_{y \in l_{n}: y \sim x} \beta(y)
$$

where $y \sim x$ denotes all the nodes in the information set $l_{k}$ of the extended game which correspond to the node $x$ in the base game.

By contruction, $(\tilde{\sigma}, \vec{\beta})$ is a reduced sequential equilibrium which induces the same payroffs as $(\sigma, \beta)$.

Now, let $(\bar{\sigma}, \bar{\beta})$ be a reduced sequential equilibrium. We transform $(\bar{\sigma}, \bar{\beta})$ into a sequential equilibrium $(\sigma, \beta)$ inducing the same payoffs, by making the following construction for every information set $h$ of the base game.

For every partition $P$, we define

$$
\sigma(P):=\sum_{\bar{a} \in \bar{A}(h, P)} \bar{\sigma}(\bar{a})
$$


where $\bar{A}(h, P)$ denotes all reduced local strategies in $\bar{A}(h)$ which choose the partition $P$.

If $\sigma(P)>0$, we define for every $X \in P$ and every $a \in A(h)$

$$
\left.\sigma_{P_{i} X}(a):=\llbracket \sigma(P)\right]^{-1} \cdot \sum_{\bar{a} \in \tilde{A}\left(h_{a}, P\right): a_{X}=a} \bar{\sigma}(\bar{a}) .
$$

Here, $\sigma_{P, X}(a)$ denotes the probability that the action $a$ is chosen if the partition $P$ has been bought and the subset $X$ has been reached.

If $\sigma(P)=0$, then for every $X \in P$ we choose $\sigma_{P, X}$ in a sequentially rational way.

Since the beliefs $\bar{\beta}$ are consistent, we can choose, by construction, corresponding consistent beliefs $\beta$ of the extended game.

In this way we obtain a sequential equilibrium $(\sigma, \beta)$ which induces the same payoffs as the reduced sequential equilibrium $(\bar{\sigma}, \bar{\beta})$.

In the proof of Theorem 4.3.1, we have seen that the information value is equal to the highest payoff for the seller which can be obtained in any sequential equilibrium of any subgame. Combining this insight with the lemma above leads to the following result.

Theorem 4.4.2 The information value is equal to the highest payoff for the seller that can be obtained in any reduced sequential equilibrium of any subgame.

A reduced sequential equilibrium which supports the information value is called aptimal.

From the theorem above, we may conclude that it is sufficient to look at reduced sequential equilibria of subgames for the computation of the information value. From now on, we denote a reduced sequential equilibria of a subgame by $(\sigma, \beta)$ instead of $(\bar{\sigma}, \bar{\beta})$.

\subsection{Properties of information value}

In the following theorem, we show that expanding the set of offered partitions leads to an increase in the information value.

Theorem 4.5.1 Let $\Gamma$ and $\bar{\Gamma}$ be two extended games induced by the same base game but with different collections $\mathcal{P}_{h}$ and $\overline{\mathcal{P}}_{h}$ of partitions offered by the seller. If $\mathcal{P}_{h} \subset \overline{\mathcal{P}}_{h}$ for every $h$ then the information value of $\tilde{\Gamma}$ is greater or equal than the information value of $\Gamma$. 
Proof. Let $(\sigma, \beta)$ be an optimal reduced sequential equilibrium of $\Gamma$ in the subgame $\Gamma(c)$. Let $\bar{c}$ be the difference between the highest and the lowest payoff at the terminal nodes of the base game. We define the price vector $c^{*}$ in the extended game $\bar{\Gamma}$ as follows. For every partition in $\mathcal{P}_{h}$, the seller charges the same prize as in $c$. For every partition in $\overline{\mathcal{P}}_{h}$ but not in $\mathcal{P}_{h}$, he charges the prize $\bar{c}+1$. By sequential rationality, it follows that no buyer will buy a partition in $\overline{\mathcal{P}}_{h} \backslash \mathcal{P}_{h}$ with positive probability. Let $\bar{\sigma}$ be the reduced BSP in $\bar{\Gamma}$ which coincides with $\sigma$ and let $\bar{\beta}$ be equal to $\beta$. Then, obviously, $(\widetilde{\sigma}, \bar{\beta})$ is a reduced sequential equilibrium (but not necessarily optimal) of $\bar{\Gamma}$ in the subgame $\Gamma(\tilde{c})$ resulting in the same expected payoff for the seller as $(\sigma, \beta)$ in $\Gamma(c)$. Therefore, the information value of $\bar{\Gamma}$ is as least as high as the information walue of $\Gamma$.

In Example 4.3 in the following section we show, however, that replacing a partition by a more informative partition can lead to a lower information value.

\section{Reservation values of partitions}

Let $(\sigma, \beta)$ be a reduced assessment, $h$ an information set of the base game controlled by player $i$ and $P$ a partition in $\mathcal{P}_{h}$. By $u_{h}^{\max }(P \mid \sigma, \beta)$ wre define the maximal expected payoff that player $i$ can achieve at information set $h$ by buying the partition $P$ at price zero, given his beliefs at $h$. By $u_{h}^{\max }(\operatorname{triv} \mid \sigma, \beta$ ) we denote the maximal expected payoff that player $i$ can get at $h$ if he chooses the trivial partition at $h$.

We define the reservation value $v_{h}(P \mid \sigma, \beta)$ of the partition $P$ at information set $h$ by

$$
v_{h}(P \mid \sigma, \beta):=u_{h^{2}}^{\max }(P \mid \sigma, \beta)-u_{\hbar}^{\max }(\operatorname{triv} \mid \sigma, \beta)
$$

Note that the reservation value of a partition depends crucially on the strategies and beliefs chosen by the buyers.

The reservation value $v_{h}(P \mid \sigma, \beta)$ is the price of partition $P$ that makes the buyer at $h$ indifferent between buying $P$ and buying no information at all and is therefore the highest price that the player is prepared to pay for $P$ if $(\sigma, \beta)$ is played.

It turns out that in an optimal reduced sequential equilibrium, a partition must be bought at a price equal to the reservation value whenever the corresponding information set is reached with positive probablity and the partition is bought with positive probability at this information set. 
Theorem 4.5.2 Let $(\sigma, \beta)$ be an optimal reduced sequential equilibrium. Then, $c_{h}(P)=v_{h}(P \mid \sigma, \beta)$ whenever $\mathbb{P}_{\sigma}(h)>0$ and $\sigma_{h}(P)>0$.

Proof. Let $(\sigma, \beta)$ be an optimal reduced sequential equilibrium in the subgame $\Gamma(c)$ and $P$ a partition at $h$ with $\mathbb{P}_{\sigma}(h)>0$ and $\sigma_{h}(P)>0$.

If $c_{h}(P)>v_{h}(P \mid \sigma, \beta)$, then the buyer at $h$ prefers 'no information' strictly over 'buying $P$ '. By sequential rationality, it follows that the buyer at $h$ should buy $P$ with probability zero, which is a contradiction.

If $c_{h}(P)<v_{h}(P \mid \sigma, \beta)$, then the buyer at $h$ prefers "buying $P$ " strictly over 'no information'. Since $P$ is bought with positive probability and the buyer at $h$ is sequentially rational, buying $P$ is an optimal choice at $h$, given $(\sigma, \beta)$. Let $\mathcal{P}_{h}^{\max }$ be the set of partitions at $h$ which maximize the expected payoff at $h$, given $(\sigma, \beta)$. Then, the prices of the partitions in $\mathcal{P}_{h}^{\max }$ can all be raised by the same small amount $\varepsilon$, such that $\mathcal{P}_{h}^{\text {max }}$ remains the set of optimal partitions. This is possible since the trivial partition, which should always have price zero, is not a member of $\mathcal{P}_{h}^{\max }$. Let $c^{*}$ be the new price vector obtained in this way. Obviously, $(\sigma, \beta)$ is a reduced sequential equilibrium in $\Gamma\left(c^{*}\right)$, but the expected payoff for the seller is higher than in $\Gamma(c)$. This is a contradiction, since $(\sigma, \beta)$ is an optimal reduced sequential equilibrium in $\Gamma(c)$.

\subsection{Examples}

By analyzing three different examples, we illustrate three less intuitive phenomena which can occur in extended games.

Example 4.1. In some cases, the information value can only be supported by reduced sequential equilibria in which a player strictly randomizes over different partitions. We illustrate this by the following example.

Consider the following base game. 


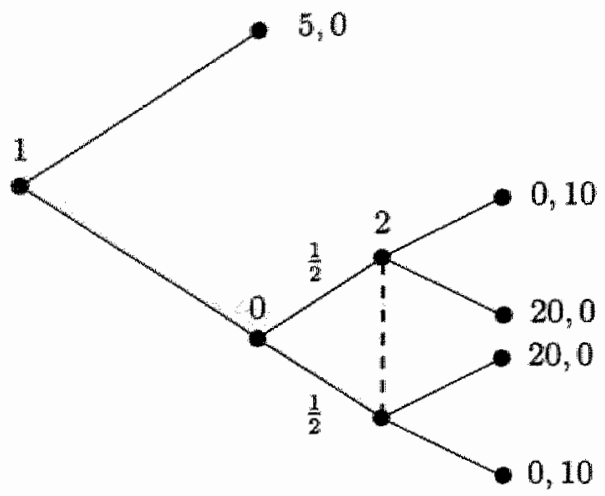

Figure 4.3

Suppose that the seller offers the trivial and the perfect partition at the information set of player 2 . We show that the information value is $\frac{5}{2}$ and prove that this payoff can only be supported by a reduced sequential equilibrium in which player 2 buys trivial and perfect information with equal probability.

Player 1 is only willing to go down if he gets at least payoff 5 at the information set of player 2. This is only possible if player 2 buys the perfect partition with probability less or equal than $\frac{1}{2}$. Since player 2 pays at most 5 for this partition, the seller can get at most $\frac{5}{2}$ in any reduced sequential equilibrium. In fact, the seller can get $\frac{5}{2}$ in the reduced sequential equilibrium where he offers the perfect partition at price 5, player 1 goes down and player 2 plays $\frac{1}{2}$ (perf, (up, down) $+\frac{1}{2}$ (triv,up) which implies that the information value is equal to $\frac{5}{2}$. Here, (perf, (up, down)) means the local strategy in which player 2 buys the perfect partition, goes up at the upper node and goes down at the lower node whereas (triv,up) denotes the local strategy in which he chooses the trivial partition and goes up.

Example 4.2. One might get the impression that it is always optimal for the seller that the buyers buy non-trivial information at each non-trivial information set. However, this is not true. In order to illustrate this, we present an example in which we prove that there is no optimal reduced sequential equilibrium in which player 2 buys non-trivial information with positive probability.

Consider the following base game. 


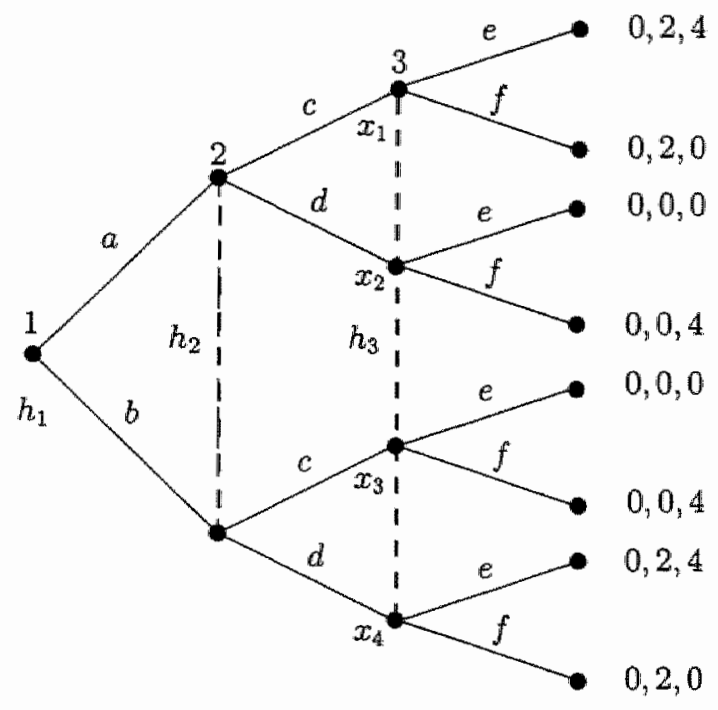

Figure 4.4

Assume that the seller offers at both information sets only the trivial and perfect partition. We show that in every optimal reduced sequential equilibrium, perfect information is bought with probability zero at $h_{2}$.

Before giving the formal proof, we try to give a verbal explanation for this phenomenon. If player 2 buys perfect information with probability one, then player 3 has no need to buy information. More precisely, if player 2 buys information with some positive probability, then this leads to a decrease in the amount that player 3 is prepared to pay for information. Since this decrease outweights the increase in the seller's income by selling information to player 2 with some positive probability, it is optimal for the seller not to sell any information to player 2 .

Now, we turn to the formal proof. Consider the price vector $c$ and the reduced assessment $(\sigma, \beta)$ in which $c_{h_{2}}$ (perf) $=1, c_{h_{3}}$ (perf) $=2$, player $\mathbb{1}$ chooses $\frac{f}{2} a+\frac{1}{2} b$, player 2 chooses (triv, $c$ ), player 3 chooses (perf, $(e, f, f, e)$ ) and the beliefs are derived by Bayes' rule. Then, it can be shown that $(\sigma, \beta)$ is a reduced sequential equilibrium in $\Gamma(c)$ in which the expected payoff for the seller is equal to 2 . So, an optimal reduced sequential equilibrium gives at least payoff 2 to the seller. 
Assume that $(\sigma, \beta)$ is a reduced sequential equilibrium in some subgame $\Gamma(c)$ such that perfect information is bought with positive probability at $h_{2}$. Let $\sigma_{h_{1}}=\alpha($ triv, $a)+(1-\alpha)$ (triv, $\left.b\right)$.

We distinguish three cases; (a) $\alpha>\frac{1}{2}$, (b) $\alpha=\frac{1}{2}$ and (c) $\alpha<\frac{1}{2}$.

(a) If $\alpha>\frac{1}{2}$, then player 2 will choose $c$ if he chooses trivial information. So, $u_{h_{2}}^{\max }(\operatorname{triv}, \sigma, \beta)=2 \alpha$ which implies that the reservation value of perfect information at $h_{2}$ is equal to $2-2 \alpha$. Since it is clear that player 2 will always choose $(c, d)$ when he buys perfect information, we may assume that player 2 plays

$$
\lambda(\text { perf },(c, d))+(1-\lambda)(\text { triv }, c)
$$

with $\lambda>0$.

Obviously, $\beta\left(x_{1}\right)+\beta\left(x_{4}\right)=\alpha+(1-\alpha) \lambda$ and $\beta\left(x_{2}\right)+\beta\left(x_{3}\right)=(1-\alpha)(1-\lambda)$. Since $\alpha>\frac{1}{2}$ we have that

$$
\begin{aligned}
\beta\left(x_{1}\right)+\beta\left(x_{4}\right) & >(1-\alpha)+(1-\alpha) \lambda=(1-\alpha)(1+\lambda) \\
& >\beta\left(x_{2}\right)+\beta\left(x_{3}\right) .
\end{aligned}
$$

Hence, player 3 will choose $e$ when having trivial information, which implies that $u_{h_{3}}^{\max }(\operatorname{triv}, \sigma, \beta)=4(\alpha+(1-\alpha) \lambda)$. Therefore, the reservation value of perfect information at $h_{3}$ is equal to $4-4(\alpha+(1-\alpha) \lambda)$.

It follows that the expected payoff for the seller is equal to

$$
\begin{aligned}
& \sigma_{h_{2}} \text { (perf) }(2-2 \alpha)+\sigma_{h_{3}}(\text { perf })(4-4(\alpha+(1-\alpha) \lambda)) \\
= & \lambda(2-2 \alpha)+\sigma_{h_{3}} \text { (perf) }(4-4(\alpha+(1-\alpha) \lambda)) \\
\leq & \lambda(2-2 \alpha)+1(4-4(\alpha+(1-\alpha) \lambda)) \\
= & \lambda(2 \alpha-2)+4-4 \alpha \\
\leq & 4-4 \alpha<2
\end{aligned}
$$

since $\alpha>\frac{1}{2}$. So, the expected payoff for the seller is strictly less than 2 if $\alpha>\frac{1}{2}$.

(b) If $\alpha=\frac{1}{2}$, player 2 is indifferent between $c$ and $d$ when having trivial information, so we assume that player 2 chooses

$$
\sigma_{h_{2}}=\lambda(\text { perf },(c, d))+(1-\lambda)(\mu(\text { triv, } c)+(1-\mu)(\text { triv, } d))
$$

with $\lambda>0$.

Since player 2 can only guarantee payoff 1 by choosing trivial information, the reservation value of perfect information at $h_{2}$ is equal to 1 .

As can be shown easily, $\beta\left(x_{1}\right)+\beta\left(x_{4}\right)=\frac{1}{2}+\frac{1}{2} \lambda$ and $\beta\left(x_{2}\right)+\beta\left(x_{3}\right)=\frac{1}{2}-\frac{1}{2} \lambda$. Since $\lambda>0$, player 3 will strictly prefer $e$ when having trivial information, 
so $u_{h_{3}}^{\max }($ triv, $\sigma, \beta)=4\left(\frac{1}{2} \lambda+\frac{1}{2}\right)$. Therefore, the reservation value of perfect information at $h_{3}$ is equal to $4-4\left(\frac{1}{2} \lambda+\frac{1}{2}\right)=2-2 \lambda$. Hence, the expected payoff for the seller is

$$
\begin{aligned}
\sigma_{h_{2}}(\text { perf }) 1+\sigma_{h_{3}}(\text { perf })(2-2 \lambda) & =\lambda 1+\sigma_{h_{3}}(\text { perf })(2-2 \lambda) \\
& \leq \lambda 1+1(2-2 \lambda)<2
\end{aligned}
$$

So, the seller gets strictly less than 2 when $\alpha=\frac{1}{2}$.

(c) By the symmetry of the extensive form game, it is clear that case (c) can be treated in exactly the same way as case (a) to show that the seller gets strictly less than 2 when $\alpha<\frac{1}{2}$.

So, it turns out that there is no optimal reduced sequential equilibrium in which perfect information is bought with positive probability at $h_{2}$.

Since the seller can not get more than 2 if perfect information is bought with probability zero at $h_{2}$, it follows that the original reduced sequential equlibrium $(\sigma, \beta)$ in $\Gamma(c)$ is optimal and the information value is equal to 2 .

Example 4.3. In this example, we show that replacing a partition by a more informative one can lead to a decrease of the information value.

Consider the following base game. 


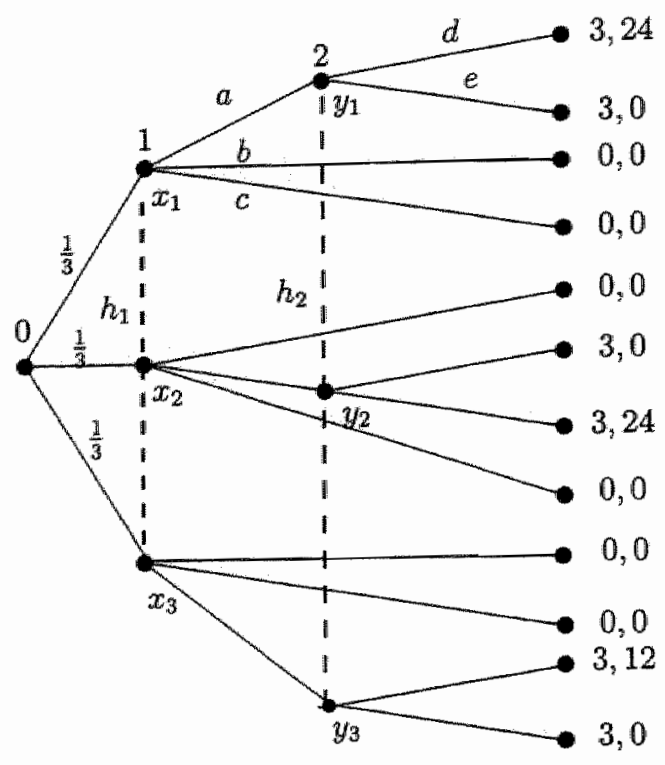

Figure 4.5

(a) Assume first that the seller offers the trivial partition and the partition $P=\left(\left\{x_{1}\right\},\left\{x_{2}, x_{3}\right\}\right)$ at $h_{1}$ and offers the trivial and perfect partition at $h_{2}$. Consider the reduced assessment $(\sigma, \beta)$ in $\Gamma(c)$ where $c_{h_{1}}(P)=1$ and $c_{h_{2}}($ perf $)=$ 12. Furthermore, let.

$$
\begin{gathered}
\sigma_{h_{1}}=(P,(a, b)), \sigma_{h_{2}}=(\operatorname{perf},(d, e, d)) \\
\beta_{h_{1}}=\left(\frac{1}{3}, \frac{1}{3}, \frac{3}{3}\right) \text { and } \beta_{h_{2}}=\left(\frac{1}{2}, \frac{1}{2}, 0\right) .
\end{gathered}
$$

Here, $(P,(a, b))$ means that player 2 will choose $a$ if he knows that the subset $\left\{x_{1}\right\}$ has been reached and he will choose $b$ if he observes that the subset $\left\{x_{2}, x_{3}\right\}$ has been reached. It can be shown that $(\sigma, \beta)$ is a reduced sequential equilibrium in $\Gamma(c)$ in which the expected payoff for the seller is $1+12=13$.

Now, let $(\sigma, \beta)$ be an arbitrary reduced sequential equilibrium in an arbitrary subgame $\Gamma(c)$. Since

$$
\begin{aligned}
& u_{k_{2}}^{\max }(\operatorname{perf} \mid \sigma, \beta)=24 \beta\left(y_{1}\right)+24 \beta\left(y_{2}\right)+12 \beta\left(y_{3}\right) \text { and } \\
& u_{k_{2}}^{\max }(\operatorname{triv} \mid \sigma, \beta)=\max \left\{24 \beta\left(y_{1}\right)+12 \beta\left(y_{3}\right), 24 \beta\left(y_{2}\right)\right\}
\end{aligned}
$$

we have that

$$
v_{h_{2}}(\text { perf } \mid \sigma, \beta)=\min \left\{24 \beta\left(y_{1}\right)+12 \beta\left(y_{3}\right), 24 \beta\left(y_{2}\right)\right\}
$$


It follows that

$$
\begin{aligned}
v_{h_{2}}(\text { perf } \mid \sigma, \beta) & \leq \frac{1}{2}\left(24 \beta\left(y_{1}\right)+12 \beta\left(y_{3}\right)+24 \beta\left(y_{2}\right)\right) \\
& =12\left(\beta\left(y_{1}\right)+\beta\left(y_{2}\right)\right)+6 \beta\left(y_{3}\right) \\
& =12\left(1-\beta\left(y_{3}\right)\right)+6 \beta\left(y_{3}\right)=12-6 \beta\left(y_{3}\right) \leq 12
\end{aligned}
$$

Moreover, it can be shown that the reservation value of $P$ at $h_{1}$ can not be more than 1 . Combining these two results, we may conclude that the expected payoff for the seller is less or equal to $1+12=13$ which implies that the information value is equal to 13 .

(b) Now assume that the seller replaces the partition $P$ at $h_{1}$ by the perfect partition.

Consider the reduced assessment $(\sigma, \beta)$ in the subgame $\Gamma(c)$ where $c_{h_{1}}$ (perf) $=$ 2 and $c_{h_{2}}($ perf $)=12, \sigma_{h_{1}}=\left(\operatorname{triv},\left(\frac{1}{2} a+\frac{1}{2} b\right)\right)$ and $\sigma_{h_{2}}=($ perf, $(d, e, d))$. It can be shown that $(\sigma, \beta)$ is a reduced sequential equilibrium in $\Gamma(c)$ which gives the seller payoff 12 .

Now, let $(\sigma, \beta)$ be an arbitrary reduced sequential equilibrium in an arbitrary subgame $\Gamma(c)$.

Let $\lambda$ be the probability that player 1 buys the perfect partition at $h_{1}$. Then, it follows that $\mathbb{P}_{\sigma}\left(y_{3}\right) \geq \frac{1}{3} \lambda$ and $\beta\left(y_{3}\right) \geq \mathbb{P}_{\sigma}\left(y_{3}\right) \geq \frac{1}{3} \lambda$.

In (a), we have seen that the reservation value of perfect information at $h_{2}$ is less or equal to $12-6 \beta\left(y_{3}\right)$. Hence, this reservation value is less or equal to $12-2 \lambda$. Therefore, the reservation value of any partition at $h_{2}$ is less or equal to $12-2 \lambda$, which implies that the seller can not gain more than $12-2 \lambda$ by selling information at $h_{2}$.

It can be shown that the reservation value of perfect information at $h_{1}$ can not be more than 2 . Since perfect information at $h_{1}$ is bought with probability $\lambda$, it follows that the seller cam not gain more than $\lambda 2$ by selling information at $h_{1}$.

Combining the two results above, we may conclude that the expected payoff for the seller is less or equal to $\lambda 2+(12-2 \lambda)=12$, which implies that the information value is equal to 12 .

\subsection{Public information transaction}

In this section, we investigate how the information value changes if we assume that the transaction of partitions bought at an information set is observed by all buyers following this information set. By providing two examples, it is 
shown that this assumption can either increase or decrease the information value: 1

Formally, assuming that transaction of information is publicly observed transforms each base game into a new extended game. In a similar way as in the 'standard' situation, where information transactions are not observed by the other buyers, it can be shown that every extended game in the new situation contains a sequential equilibrium and the sellers payoff obtains a maximum on the set sequential equilibria. Therefore, a definition of the information value being the highest payoff that the seller can receive in a sequential equilibrium, is possible. In the following example, we show that the information value can increase by making information transactions public.

Example 4.4. Consider the following base game.

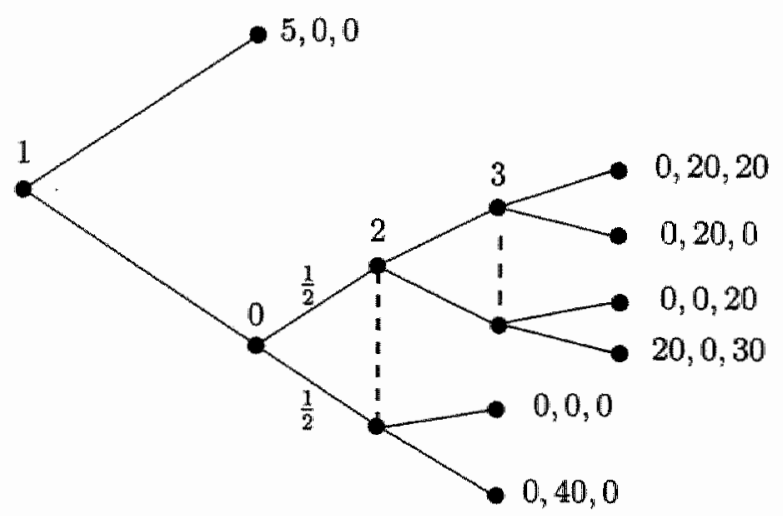

Figure 4.7

Suppose that the seller offers trivial and perfect information to player 2 and offers only trivial information to player 3 . We show that the information value in the standard situation (with privately observed information transactions) is $\frac{10}{3}$ whereas the information value in the new situation (with publicly observed information transactions) is equal to 5 .

We start with the standard situation. Note that player 1 will only go down if, with probability at least $\frac{1}{2}$, player 3 goes down at his lower node. Player 3

\footnotetext{
II am grateful to Michael Maschler, who encouraged me to investigate the case of publicly obserwed information transaction.
} 
will only do this if his belief at the lower node is at least $\frac{2}{3}$. Since player 2 will only go down at his upper node if he buys trivial information, it follows that player 1 will only go down if player 2 buys trivial information at least with probability $\frac{2}{3}$. So, perfect information is bought at most with probability $\frac{1}{3}$. Since player 2 will not pay more than 10 for perfect information, the seller can not expect more than $\frac{1}{3} \cdot 10$ in a reduced sequential equilibrium.

However, the seller can get $\frac{10}{3}$ if he chooses price 10 for perfect information, player 1 goes down, player 2 plays $\frac{1}{3}$ (perf, (up,down)) $+\frac{2}{3}$ (triv,down) and player 3 goes down. This is a reduced sequential equilibrium in the subgame following price 10 . Therefore, the information value is equal to $\frac{10}{3}$.

Now, consider the new situation. Again, player 1 will only go down if, with probability at least $\frac{1}{2}$, player 3 goes down at his lower node. Since player 2 will go up at his upper node up after buying perfect information and go down at his upper node after buying trivial information, player 3 will go down only after observing that player 2 has bought trivial information. Since player 3 should go down at least with probability $\frac{1}{2}$, player 2 should buy trivial information with probability at least $\frac{1}{2}$. So, perfect information is bought at most with probability $\frac{1}{2}$ if player 1 goes down. Since player 2 will not pay more than 10 for perfect information, the seller can not expect more than $\frac{1}{2} \cdot 10$.

However, the seller can get 5 in the reduced sequential equilibrium in the subgame following price 5 where player 1 goes down, player 2 plays $\frac{1}{2}$ (perf,(up,down)) $+\frac{1}{2}$ (triv,down), player 3 goes up after observing that 2 bought perfect information and goes down after observing that 2 bought trivial information. Hence, the information value is 5 .

The intuition behind the increase of the information value is the fact that, in this situation, it is beneficial for the seller that player 3 knows which information has been bought by player 2. However, the example below shows that the reverse can also occur: public information transaction can lead to a decrease of the information value.

Example 4.5. Consider the following base game. 


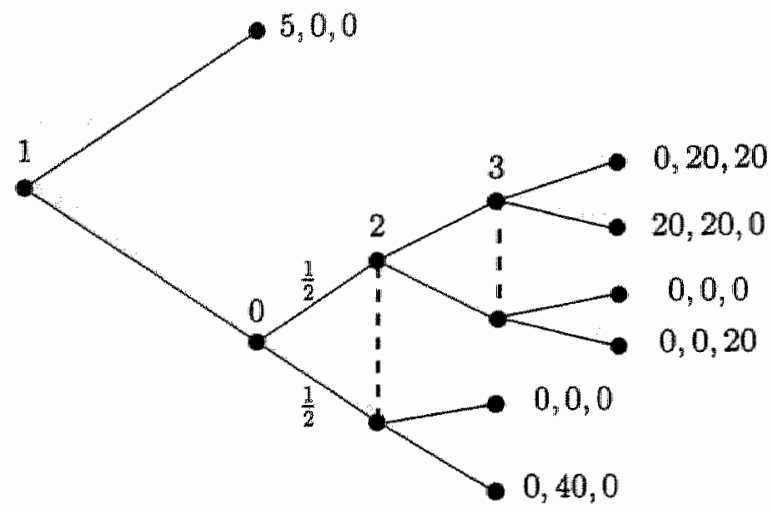

Figure 4.8

Suppose, again, that the seller offers trivial and perfect information to player 2 and only trivial information to player 3 . We show that the information values in the standard and the new situation are 5 and 0 respectively.

In the standard situation, player 1 will only go down if player 3 will go down at his upper node with with probability at least $\frac{1}{2}$. Player 3 is only willing to go down if player 2 goes down at his upper node at least with probability $\frac{1}{2}$. Since player 2 will only go down at his upper node after buying trivial information, it follows that player 2 should buy trivial information at least with probability $\frac{1}{2}$. Hence, perfect information is bought at most with probability $\frac{1}{2}$. Player 2 will not be paying more than 10 for perfect information, so the seller can expect at most $\frac{1}{2} \cdot 10$.

The seller can get 5 in the reduced sequential equilibrium of the subgame following the price 5 where player 1 goes down, player 2 plays $\frac{1}{2}$ (perf,(up,down)) $+\frac{1}{2}($ triv, down $)$ and player 3 goes down. This implies that the information velue is 5 .

Now, consider the new situation. If player 3 observes that player 2 has bought perfect information, he knows that player 2 has gone up at his upper node and player 3 will go up, leaving player 1 with payoff 0 . However, if player 2 buys trivial information, player 2 will go down giving player 1 payoff 0 . Hence, player 1 will always get 0 if he goes down. Therefore, player 1 will always go up in a reduced sequential equilibrium which implies that the information value is 0 . 
In the latter example, it hurts the seller if player 3 knows which information has been bought by player 2 .

\subsection{Non-single appearance games}

In this section we discuss the difficulties which arise if we want to extend the model to base games which are non-single appearance games, i.e. games in which at least one player appears more than once on a certain path in the game tree.

Consider, for example, the following non-single appearance game in which player 2 appears two times on the same path.

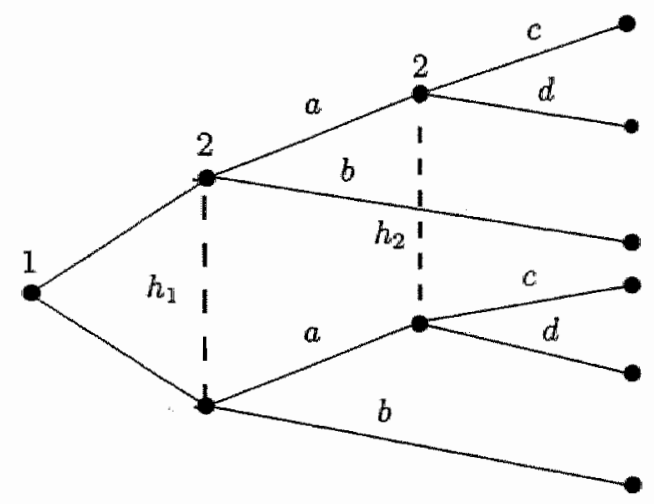

Figure 4.9

If player 2 would buy perfect information at $h_{1}$, perfect recall implies that player 2 would automatically have perfect information at $h_{2}$. In other words, the information set $h_{2}$ will be split into singletons whenever player 2 buys perfect information at $h_{1}$.

More generally, the actual structure of an information set depends on the information bought at previous information sets of the same buyer.

If player 2 buys perfect information at $h_{1}$ in the example above, the information set $h_{2}$ will be split in two singleton information sets $h_{2 a}$ and $h_{2 b}$ and the seller can only offer trivial information at these information sets.

However, if player 2 would buy trivial information at $h_{1}$, the information set 
$h_{2}$ 'remains as it is' and the seller can offer trivial and perfect information at $h_{2}$.

Hence, it is clear that the situation at $h_{2}$ can not be handled independently from what happened at $h_{1}$. It is this compatibility problem that makes the non-single appearance case so complex.

In the definition of a BSP in the single appearance case, every player can randomize over his set of reduced local strategies at each of his information sets, independent of what he does at the other information sets. In a single appearance base game, this can not cause troubles, since the decision of a player at information set $h$ does not affect the set of reduced local strategies at his other information sets.

However, in a non-single appearance base game, it is not possible to let a player randomize over his reduced local strategies at each information set, independent of his behavior at other information sets since this may cause compatibility problems for reasons discussed above.

A possible way to solve this problem is to split the collection $H_{i}$ of information sets controlled by player $i$ into a maximal number of sub-collections $H_{i j}$ such that on every path of the game tree, at most one subcollection $H_{i j}$ of every player $i$ is crossed.

If $\Gamma$ would be a single appearance game, the subcollections $H_{i j}$ would simply be the individual information sets.

For every sub-collection $H_{i j}$ we define $\bar{A}\left(H_{i j}\right)$ to be the set of reduced local strategy profiles $\left(\vec{a}_{h}\right)_{h \in H_{i j}}$ such that the reduced local strategies in this profile respect perfect recall.

In the example above, $H_{2}$ is split into only one collection $H_{2,1}$. The re-

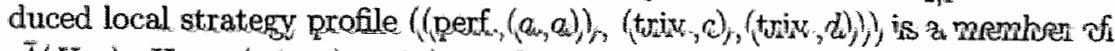
$\vec{A}\left(H_{2,1}\right)$. Here, (triv, $\left.c\right)$ and (triv, $\left.d\right)$ are the actions chosen at the singleton information sets $h_{2 a}$ and $h_{2 b}$ respectively.

The reduced local strategy profile ((perf, $(a, a)$, (perf), $(c, d)))$, where (perf), $(c, d))$ is the reduced local strategy chosen at $h_{2}$ is not a member of $\vec{A}\left(H_{2,1}\right)$ since it does not respect perfect recall.

We can define a BSP by allowing every player $i$ to randomize over each of his sets $\bar{A}\left(H_{i j}\right)$. Note that the decisions made in different collections $H_{i j}$ and $H_{i k}$ have no influence on their respective sets of available reduced local strategy profiles. Therefore, we allow for independent randomization on the sets $\bar{A}\left(H_{i j}\right)$. If $\Gamma$ is a single appearance game, we end up with the "classical" BSP as defined in the single appearance case.

In the same way as in the single appearance case, we can define belief systems, sequential equilibria and reduced sequential equilibria. With similar meth- 
ods, we can show that sequential equilibria always exist and the payoff for the seller reaches a maximum on the set of sequential equilibria. Therefore, the information value can also be defined for non-single appearance games. 



\section{Chapter 5}

\section{Buying information and the seller's commitment degree}

\subsection{Introduction}

This chapter is based on Perea y Monsuwé and Swinkels (1997).

The problem of reaching decisions under uncertainty is a phenomenon which occurs in many economic situations. In quite a number of these situations, there is an 'outsider' who has access to relevant information about uncertain elements and can sell this information to the decision makers. For instance, a consultant may have access to data about outputs in a duopoly. An expert may know more about quality than the players in a signalling game. A spy may have information about the moves in a strategic interaction between two firms and so on. In economic and game theoretic literature, a lot of attention has been paid to situations where decision makers can extract additional information from an outsider before reaching a decision.

For example Sakai (1985) considers a duopoly in which the two firms face uncertainty about their own marginal costs and the marginal costs of the opponent. However, there is a market research agency which can provide both firms with information about these uncertain facts. In this paper, Sakai investigates how the additional information provided by the agency influences the behavior of the firms. In Admati and Pfeiderer (1986), traders on a speculative market can buy private information about the payoff of a risky asset from a monopolistic information seller. Kamien et al. (1990) explores the situation in which an outsider can provide the players of an extensive form game with relevant information about the play of the game. More papers in this field are for instance Allen (1986), Levine and Ponssard (1977), Milne and Shefrin (1987) and Green (1981), to mention just a few. 
In nearly all these settings, the information holder is non-strategic: he is present to provide decision makers with additional information but does not have strategic motivations for his behavior. However, it seems relevant in most of the examples above to consider the incentives of the information seller as well. The presence of an information seller induces in many cases an interaction between him and the decision makers, from now on called information buyers. The information that will be offered and the price that is charged for this depends crucially on the behavior of the buyers, whereas the information that will be bought by the buyers and the price that the buyers are prepared to pay for this depends on the behavior of the seller and the behavior of the other buyers. It is this strategic interaction that we want to explore in this chapter. More specifically, we focus on the question how the maximum income of the information seller changes if we change his degree of commitment in this strategic interaction.

In the previous chapter, we examined the situation of a strategic information seller. Starting from a so-called 'base game', a player is added who has access to some information about the play of the game. This might be information about a choice by nature or about the moves of one or more of the players. This player, who plays the role of information seller, has the ability to sell this information to the players of the base game. The seller may choose to sell all or only part of his information. The key question is then "what information should he sell and how much should he charge?"

This question is answered by studying the following model. At each of the information sets in the base game, the seller offers a collection of partitions dividing the information set into disjoint subsets of nodes. Hence, information is implemented formally by partitions. Before the actual game starts, the seller chooses a price for every partition that is offered. This menu of prices is revealed to all buyers. Subsequently, the buyers start playing the base game with the additional feature that the partitions can be bought against the prices specified in the menu.

Important in this model is the degree of commitment on the part of the seller. By commitment, we mean the possibility for the seller to make binding agreements about future decisions which, at that future point of the game, would be suboptimal for the seller. In the model of the previous chapter, the seller can make binding agreements about the prices that he will charge at a certain information set, although these prices might not be optimal once this information set is reached. To see this, consider the following example. 


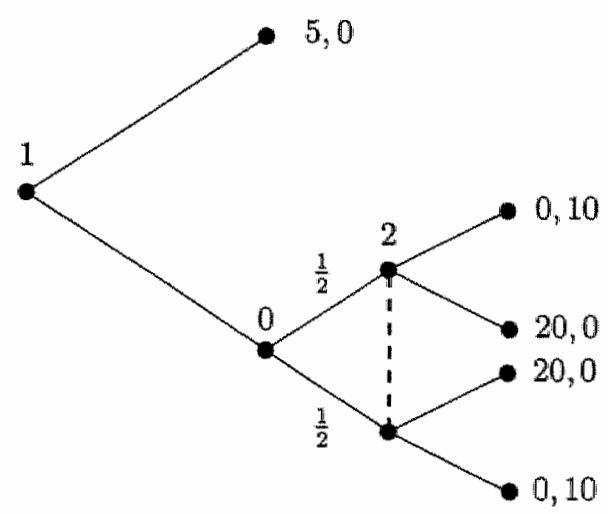

In this game, player 1 will avoid player 2's information set if he knows that player 2 will buy information with probability one. In this case, the seller is left with no income. On the other hand, if player 2 does not buy information, the seller does not make any money either. The optimal situation for the seller would be to offer information at price 5 and having player 2 to randomize equally between buying and not buying. In this case, player 1 is prepared to go down and the seller will have income $\frac{5}{2}$. However, once the information set of player 2 is reached, the seller would be better off to offer information at a somewhat lower price since this would force player 2 to buy information for sure and give the seller an income higher than $\frac{5}{2}$. So, the partial commitment power helps the seller to receive an income of $\frac{5}{2}$.

This income would not be possible without commitment power. In this case, sequential rationality at player 2 's information set would force the seller to sell information with probability one at price 5 . However, if player 1 knows this, he will go up and leave the seller with payoff zero. So, without commitment power, the seller can not expect any income.

Conversely, assume the seller has more commitment power than in the previous chapter. For example, consider the situation where the seller commits himself not to sell any information to player 2, player 1 goes down and player 2 goes up. In this case, player 1 and 2 receive payoffs 10 and 5 respectively. However, the seller can charge 5 to both players for not selling information by threatening to sell information if they do not pay this price, leaving the players with payoffs 5 and 0 respectively. Hence, the seller can obtain income 10 by exploiting his commitment power in this situation.

So, the example shows that the revenues of the seller clearly depend significantly on the degree of commitment the seller posesses. The main purpose of this chapter is to formalize the seller's degree of commitment and to explore its influence on his revenue. 
We begin by laying out the model in which we implement the process of buying information and define the so-called information value as the highest revemue that can be obtained by the seller, given that seller and buyers play a sequential equilibrium. However, the information value depends crucially on the commitment degree of the seller in this model. In the remainder of the chapter, we analyze three different situations which will be called the case of no commitment, the case of full commitment and the standard case. In the case of no commitment, the seller cannot promise any buyer that he will not later sell to another buyer, nor can he make credible statements about what information he has already sold. This is implemented by assuming that, each time a buyer finds himself at an information set, the seller must choose prices for the partitions offered at this information set. Since we require sequential rationality for the seller at each information set, he can not commit to act in a suboptimal way at later stages of the game in order to raise his income. The information transactions are unobserved by later players, and no agreements can be reached about later information revelations.

In this setting, we begin by demonstrating existence of a sequential equilibrium. The reason there is an issue here is because the information seller can choose from a continuum of prices at each information set. Furthermore, it is shown that the seller's income reaches a maximum on the set of sequential equilibria and therefore the information value is well-defined in this case.

We then turn to the case of full commitment. We want to consider a situation in which the information seller has available a rich set of negotiation strategies and can make binding commitments about what information he will and won't sell and the prices against which the information will be sold. So, for example, the seller can contract with player 1 at the beginning of the game not to sell information to player 2. Or, the seller can contract with player 2 that should a certain information set be reached, player 2 will receive information for free. Or, the seller can set up an auction between players 1 and 2 in which the highest bidder gets to choose which of these two information regimes will be implemented. On the other hand, we want to focus on the role of an information seller, and not for example a Mafia enforcer or an insurance seller. For example, we do not want to allow contracts in which the amount that a player pays for information depends on the action he takes having received the information. Doing so would allow the information seller to take on the role of enforcer. Similarly, a contract with a player that bases payments on the outcome of the game or the node within an information set at which a player actually is, could easily expand the role of the information seller to that of insurance agent. Moreover, we want to enforce a condition that players are voluntary purchasers of information. To do so, we impose the condition that, whatever the form of negotiations, every buyer has avail- 
able a behavior which guarantees himself that he pays nothing to the seller. Finally, we assume that, before the actual game starts, all buyers are aware of the partitions that will be offered by the seller and their corresponding prices.

Still this allows for a very broad and particularly intractable specification of behaviors. Even defining the full spectrum of available behaviors seems quite challenging. However, we are able to establish an upper bound of the sellers payoff in this situation. In order to see this, observe that in the end, all the information seller can have done in the play of a game is to have extracted some money from some subset of buyers and provided some information to some (possibly different) subset of buyers. Since buyers play a sequential equilibrium after every outcome of the negotiation phase, the highest sum of payoffs available across the buyers and the seller is at most the highest sum of payoffs attainable as a sequential equilibrium of a game generated from the original game by changing the information structure and choosing particular prices for the partitions. On the other hand, a buyer can always refuse to take part in negotiations with the seller. So, he can always guarantee himself his smallest payoff among the set of all possible sequential equilibria. This payoff can be seen as a individually rational payoff for the buyer. These two observations imply that the seller can never expect more than the difference between the highest sum of payoffs (including the seller's payoff) obtainable in a sequential equilibrium and the sum of individually rational payoffs among the buyers.

Moreover, we show that this upper bound is sharp: the seller can actually obtain this payoff by constructing a fairly simple negotiation phase which is called the standard negotiation phase. In this negotiation phase, the seller first charges each buyer some price. Subsequently, the buyers simultaneously decide whether or not to pay the price. After having observed the answers of the buyers, the seller signs a contract in which he specifies the prices which he will charge for the partitions. By doing this, the seller can effectively punish a buyer for not paying the price by selecting those prices for partitions which are worst for the buyer, and therefore leaving the buyer with his individually rational payoff. In this way, we obtain a characterization of the information value in the case of full commitment.

Next, we briefly discuss the standard case, in which the information seller has to choose a menu of prices before the actual game starts. For an extensive analysis of the standard case, we refer to the previous chapter.

Finally, we analyze the influence of the seller's commitment power on his revenue by comparing the information values of the three cases mentioned above. It turns out that the information value of the no commitment case is always less than or equal to the value of the standard case which, in turn, can 
never be more than the value in the full commitment case. This result can be seen as a formal proof of the intuitive phenomenon that more commitment power leads to a higher payoff for the seller.

The outline of the chapter is as follows. In section 2 , we describe how the process of buying information is formally implemented and we give a definition of the information value. Section 3 analyzes the case of no commitment. Moreover, we show that node-dependent prices can never be beneficial for the seller. In section 4, the case of full commitment is explored and a characterization of the information value in this case is given. The standard case is briefly mentioned in section 5 whereas section 6 compares the information values of the three different cases. We conclude the chapter with an example.

\subsection{Buying information and information value}

In this section, we describe how the process of buying information is formally implemented. In spite of the fact that the role of the seller differs among the different situations we consider, the implementation of the information transaction is the same in each of the situations.

\section{Buying information}

We consider a situation in which the players of an extensive form game, which in the remainder of this chapter will be called the base game, have the opportunity to buy additional information at each of their information sets. We require that the base game is a single appearance game with perfect recall. By a single appearance game, we mean a game in which every player appears at most once in every play of the game.

The process of buying information is formalized by assuming that a monopolistic information seller offers a certain collection of partitions at every information set, which divide the nodes in the information set into disjoint subsets. If a player buys a particular partition at one of his information sets, he receives a signal from the seller which reveals the subset of the partition reached by the play of the game. We assume that the trivial partition, revealing no additional information, is offered at each information set. Moreover, the partition bought by a buyer is not observed by the other buyers.

Since information is costly, the players must pay a price for it. The price of information is determined by the information seller. However, there are many different ways to implement this process of price setting. The seller could, for instance, choose all the prices before the actual game starts or choose 
the price of a partition at the moment that the corresponding information set is reached. As a natural condition, we impose that the trivial partitions should always be offered at price zero, meaning that a buyer always has the opportunity to refrain from buying information. Every choice of the process of price setting gives rise to a new game which we call the extended game. In this chapter we investigate how the process of price setting influences the behavior of the players in the resulting extended game.

The game tree of such an extended game is typically very large. Look, for example, at the following fragment of a base game.

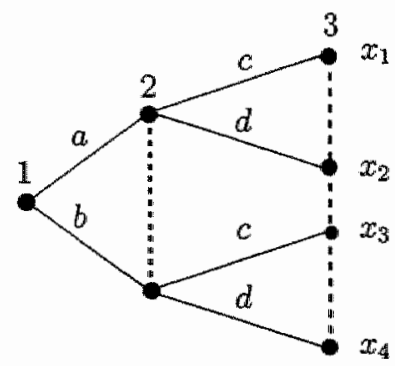

Figure 5.1

Suppose that player 2 can choose between the trivial partition (revealing no information) and the perfect partition (revealing perfect information) at his information set. The corresponding fragment of the extended game would look as follows. 


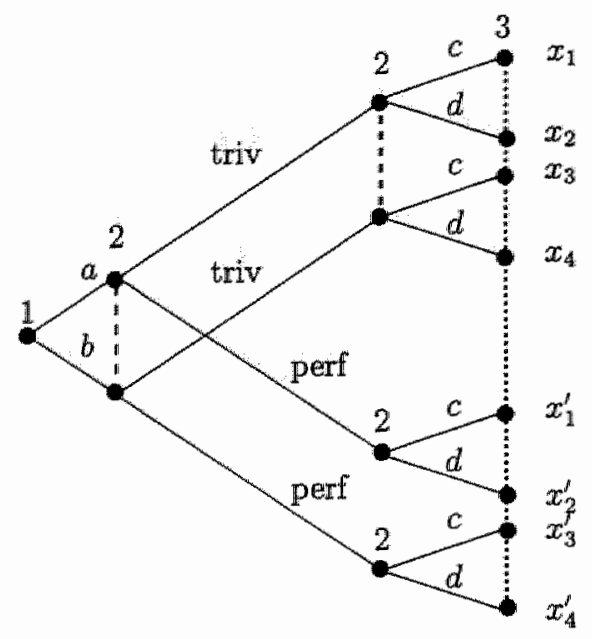

Figure 5.2

Here, the number of information sets for player 2 has increased from 1 to 4. Moreover, the information set of player 3 has been duplicated since he does not know which partition has been chosen by player 2 . This simple example already shows that the number of information sets and the size of the information sets can grow enormeously, especially if the number of partitions is large. As a consequence, the size of formal behavior strategies and beliefs for the extended game is in general unfortunately large. However, the extended game contains a lot of redundant information which allows us to use a much more compact definition of behavior strategies and beliefs.

Suppose, for instance, that player 2 chooses the trivial partition in the example. For the analysis of the game, it does not matter which actions player 2 would have chosen if he had chosen the perfect partition. Therefore, the behavior for player 2 can be described by a vector $\left(P,\left(a_{X}\right)_{X \in P}\right)$ where $P$ is the partition chosen by player 2 and $a_{X}$ is the action that will be chosen if subset $X \in P$ is revealed. The vector $\left(P,\left(a_{X}\right)_{X \in P}\right)$ is called a reduced local strategy. The set of reduced local strategies at an information set. $h$ is denoted by $\bar{A}(h)$.

In the example, the extensive form structures following the nodes $x_{i}$ and $x_{i}^{\prime}$ are the same. Moreover, player 2 does not appear in the game which follows the information set of player 3 since we assumed that the base game is a single 
appearance game. Obviously, the games following $x_{i i}$ and $x_{i}^{\prime}$ have the same terminal payoffs for all players except player 2. Therefore, the nodes $x_{i}$ and $x_{i}^{\prime}$ can be treated as identical nodes in the continuation of the game which implies that the information set of player 3 can be reduced to its original size for the analysis of the continuation of the game. In particular, the beliefs of player 3 can be represented by a vector $\beta=\left(\beta\left(x_{i}\right)\right)_{i=1,2.3 .4}$ of size 4 in which $\beta\left(x_{i}\right)$ is equal to $\bar{\beta}\left(x_{i}\right)+\bar{\beta}\left(x_{i}^{\prime}\right)$. Here, $\bar{\beta}$ are the formal beliefs at the 'large' information set of player 3 in the extended game. In general, the beliefs of a player at an information set of the extended game can always be represented by the beliefs of the corresponding information set of the base game.

Therefore, assessments in the extended game can be defined completely in terms of the base game, which makes the analysis much easier.

\section{Information value}

Depending on the process of price setting by the seller, we obtain an extended game, which is an extensive form game played by the seller and the buyers. We define the information value to be the highest payoff that can be obtained by the seller in a sequential equilibrium of the extended game. Intuitively, the information value reflects the maximum amount of money that the seller can extract from the buyers by selling information, provided that seller and buyers act in a rational way.

\subsection{Case of no commitment}

First, we consider the situation in which the prices of the partitions offered at an information set are chosen at the moment this information set is reached. Since sequential rationality implies that the information seller has to act optimally at every information set, he can not commit himself to sub-optimal behavior at some information sets in order to increase his income. This is the reason to call this situation the no commitment case.

\subsubsection{Extended game, strategies and beliefs}

We start with a base game $\Gamma$. At every information set $h, \mathcal{P}_{h}$ is the collection of partitions at $h$ offered by the seller.

The extended game is an extensive form game with $n+1$ players with player $n+1$ being the information seller. Whenever an information set $h$ is reached, the information seller chooses a price $c_{h}(P)$ for every partition $P \in \mathcal{P}_{h}$. The 
trivial partition should always be offered at price zero. The prices $c_{h}(P)$ at $h$ are only observed by the player who controls $h$.

After having observed the prices $c_{h}(P)$, the player at $h$ chooses a partition $P \in \mathcal{P}_{h}$, pays the price $c_{h}(P)$ to the information seller and receives the signal indicating which subset $X \in P$ has been reached. If he observes that the subset $X$ has been reached, he chooses a corresponding action $a_{X} \in A(h)$. The actual partition $P$ chosen by the player is not revealed to the other players.

At the end of the game, the expected payoff for the seller is the expected income of selling information. The expected payoff for a buyer is the difference between the expected payoff at the terminal nodes of the base game and the expected amount to be paid for buying information.

A behavior strategy for the seller is a vector $\mu=\left(\mu_{h}\right)_{h \in H}$ which assigns to every information set $h$ a probability measure $\mu_{h}$ on the set of possible price vectors $\left(c_{h}(P)\right)_{P \in P_{h}}$ at $h$. A price vector at $h$ is denoted by $c_{h}$. The set of possible price vectors at $h$ is given by $C_{h}$.

Implicit in this definition is the assumption that the seller can not make his price vector contingent on the node which has been reached. Later, we show that this restriction is not relevant since the information seller can never benefit from making the prices dependent on the node.

A behavior strategy profile for the buyers is a vector $\sigma=\left(\sigma_{h}\right)_{h \in H}$ which prescribes for each information set $h$ a function $\sigma_{h}: C_{h} \rightarrow \Delta(\bar{A}(h))$, where $\bar{A}(h)$ is the set of reduced local strategies at $h$. So, $\sigma_{h}$ assigns to every possible price vector which can be observed at $h$ a randomization $\sigma_{h}\left(c_{h}\right)$ over the reduced local strategies at $h$.

A belief system is a vector $\beta=\left(\beta_{h}\right)_{h \in H}$ assigning to every information set $h$ a probability measure $\beta_{h}$ on $h$. Formally, at each information set $h$ we should define beliefs $\beta_{h}\left(c_{h}\right)$ after every possible price vector $c_{h} \in C_{h}$ which could have been observed by the player at $h$. Every belief $\beta_{h}\left(c_{k}\right)$ is a probability measure on the nodes in some information set $h\left(c_{h}\right)$, which is a copy of the information set $h$. The only difference between the copies $h\left(c_{h}\right)$ is the fact that they are preceded by different price vectors $c_{h} \in C_{h}$. All the nodes in one particular copy $h\left(c_{k}\right)$ are preceded by the same price vector. Therefore, consistency of the assessment implies that the beliefs $\beta_{h}\left(c_{h}\right)$ corresponding to the same information set $h$ should not depend on the price vector $c_{h}$. Consequently, it is sufficient to define one belief vector $\beta_{h}$ for every information set $h$ of the base game.

The behavior strategy profile $(\mu, \sigma)$ induces a probability distribution on the nodes in the base game. Therefore, Bayes rule can be applied in a natural way to define consistency of the assessment $(\mu, \sigma, \beta)$, similarly to the well-known 
definition by Kreps and Wilson (1982).

The assessment $(\mu, \sigma, \beta)$ is called sequentially rational if at every information set both the information seller and the corresponding information buyer maximize their expected payoff, given the beliefs and the behavior at the other information sets.

We call $(\mu, \sigma, \beta)$ a sequential equilibrium if it is both consistent and sequentially rational.

\subsubsection{Information value}

First, we show that the set of sequential equilibria is always nonempty. A sequential equilibrium is called optimal if it yields the highest expected payoff for the information seller among the set of all sequential equilibria. We prove that an optimal sequential equilibrium always exists by showing that the payoff for the seller obtains a maximum on the set of sequential equilibria. Therefore, the information value is well defined in this case.

Theorem 5.3.1 The set of sequential equilibria is always nonempty and an optimal sequential equilibrium always exists.

Proof. Let $\Gamma$ be a base game and $\Gamma^{*}$ the corresponding extended game in which the information seller offers the collection $\mathcal{P}_{h}$ of partitions at each information set $h$.

First, we show that we can find a sequential equilibrium $(\mu, \sigma, \beta)$ in $\Gamma^{*}$.

Let $\Gamma^{0}$ be the game in which all partitions are offered at price zero. Since $\Gamma^{0}$ is a finite extensive form game only played by buyers, we can find a sequential equilibrium $\left(\sigma^{0}, \beta^{0}\right)$ for the buyers in $\Gamma^{0}$. Here, $\sigma^{0}$ and $\beta^{0}$ have a similar form as in the extended game $\Gamma^{*}$. The only difference lies in the fact that the prices are now fixed, which means that for a given information set $h, \sigma_{h}^{0}$ does not. depend on the price vector at $h$.

For an information set $h$, the set of reduced local strategies which choose the partition $P \in \mathcal{P}_{h}$ is called $\vec{A}(h, P)$. Furthermore, we define

$$
u_{h}^{\max }\left(P \mid \sigma^{0}, \beta^{0}\right):=\max _{\bar{a} \in \bar{A}(h, P)} \sum_{x \in h} \beta_{h}(x) u_{i}\left(\sigma^{0} \backslash \bar{a} \mid x\right)_{n}
$$

where $\sigma^{0} \backslash \bar{a}$ is the behavior strategy profile in which player $i$ chooses the reduced local strategy $\bar{a}$ at $h$ and at all other information sets the players play according to $\sigma$. By $u_{i}\left(\sigma^{0} \backslash \bar{a} \mid x\right)$ we mean the expected payoff for player $i$ if the node $x$ has been reached and $\sigma^{0} \backslash \bar{a}$ is played. In words, $u_{h}^{\max }\left(P \mid \sigma^{0}, \beta^{0}\right)$ 
is the highest expected payoff that player $i$ can achieve at $h$ by choosing the partition $P$.

By $u_{h}^{\max }\left(\operatorname{triv} \mid \sigma^{0}, \beta^{0}\right)$ we denote the highest expected payoff that player $i$ can realize at $h$ if he chooses the trivial partition. We define the reservation value $v_{h}\left(P \mid \sigma^{0}, \beta^{0}\right)$ of $P$ by

$$
v_{h}\left(P \mid \sigma^{0}, \beta^{0}\right)=u_{h}^{\max }\left(P \mid \sigma^{0}, \beta^{0}\right)-u_{h}^{\max }\left(\text { triv } \mid \sigma^{0}, \beta^{0}\right) .
$$

So, $v_{h}\left(P \mid \sigma^{0}, \beta^{0}\right)$ is the highest price that player $i$ is prepared to pay for $P$ if he has beliefs $\beta_{h}$ and the other players play according to $\sigma^{0}$. This is exactly the price which makes him indifferent between buying $P$ and buying no information at all.

We define the assessment $(\mu, \sigma, \beta)$ of the extended game $\Gamma^{*}$ as follows. For every information set $h$, let $c_{h}^{*}$ be the price vector given by $c_{h}^{*}(P)=v_{h}\left(P \mid \sigma^{0}, \beta^{0}\right)$ for every $P \in \mathcal{P}_{h}$. Let $\mu_{h}$ be the probability measure which puts probability one on $c_{h}^{*}$. Furthermore, we define $\sigma_{h}\left(c_{h}\right)=\sigma_{h}^{0}$ if $c_{h}=c_{h}^{*}$ and for $c_{h} \neq c_{h}^{*}$ we choose $\sigma_{h}\left(c_{h}\right)$ in such a way that it is sequentially rational. Finally, we choose $\beta=\beta^{0}$.

By choice of the prices $c_{h}^{*}(P)$, the information buyers are indifferent between any partition they can buy at each of their information sets. Therefore, $(\mu, \sigma, \beta)$ is seqentially rational for the information buyers. Also, the information seller acts sequentially rational since he charges for every partition $P$ the highest price which the corresponding player is willing to accept in $(\sigma, \beta)$. Since $(\mu, \sigma, \beta)$ is consistent, we may conclude that it is a sequential equilibrium in $\Gamma^{*}$.

Now, we must show that there is an optimal sequential equilibrium in $\Gamma^{*}$.

Let $S E$ be the set of all sequential equilibria in $\Gamma^{*}$ and $u_{n+1}(S E)$ the set of expected payoffs for the information seller induced by sequential equilibria. We show that $u_{n+1}(S E)$ has a maximum.

Let $c$ be the difference between the highest and the lowst payoff at the terminal nodes of the base game $\Gamma$. Since no player is willing to pay more than $\bar{c}$ for any partition in a sequential equilibrium in $S E$, it follows that $u_{n+1}(S E)$ is bounded. Therefore, there is a supremum $\bar{u}_{n+1}$ of $u_{n+1}(S E)$. In order to show that $\bar{u}_{n+1}$ is a member of $u_{n+1}(S E)$, we consider a non-decreasing sequence $\left(\bar{u}_{n+1}^{k}\right)_{k \in \mathbb{N}}$ in $u_{n+1}(S E)$ which converges to $\bar{u}_{n+1}$. Let $\left(\mu^{k}, \sigma^{k}, \beta^{k}\right)$ be the sequential equilibrium which supports $u_{n+1}^{k}$.

Every sequential equilibrium $(\mu, \sigma, \beta)$ of $\Gamma^{*}$ can be transformed into an assessment $\left(\sigma^{0}, \beta^{0}\right)$ of the game $\Gamma^{0}$ in which all prices are zero. The assessment $\left(\sigma^{0}, \beta^{0}\right)$ is defined by

$$
\sigma_{h}^{0}=\int_{c_{h}} \sigma_{h}\left(c_{h}\right) d \mu_{h}
$$


for every information set $h$. Hence, $\sigma_{h}^{0}$ is the expected randormization over the reduced local strategies at $h$, given $\mu_{h}$. Furthermore, we define $\beta^{0}=\beta$. Obviously, $\sigma^{0}$ induces the same probability distribution on the nodes of the base game as $(\mu, \sigma)$.

As can be seen easily, the expected payoff for the information seller in $(\mu, \sigma, \beta)$ at an information set $h$ can not be more than $\sum_{P \in \mathcal{P}_{h}} \sigma_{h}^{0}(P) v_{h}\left(P \mid \sigma^{0}, \beta^{0}\right)$. If we define

$$
v\left(\sigma^{0}, \beta^{0}\right)=\sum_{h} \sum_{P \in \mathcal{P}_{h}} \mathbb{P}_{\sigma^{0}}(h) \sigma_{h}^{0}(P) v_{h}\left(P \mid \sigma^{0}, \beta^{0}\right),
$$

then the expected payoff $u_{n+1}(\mu, \sigma, \beta)$ for the seller in the sequential equilibrium $(\mu, \sigma, \beta)$ can not exceed $v\left(\sigma^{0}, \beta^{0}\right)$. Here, $\mathbb{P}_{\sigma^{0}}(h)$ denotes the probability that the information set $h$ is reached.

Now, let $\left(\sigma^{0, k}, \beta^{0, k}\right)$ be the assessment in $\Gamma^{0}$ induced by $\left(\mu^{k}, \sigma^{k}, \beta^{k}\right)$. Since the space of assessments for the finite extensive form game $\Gamma^{0}$ is compact, we may assume w.l.o.g. that the sequence $\left(\sigma^{0, k}, \beta^{0, k}\right)$ converges to some assessment $\left(\sigma^{0}, \beta^{0}\right)$. Since $v\left(\sigma^{0, k}, \beta^{0, k}\right)$ depends contimuously on the assessment $\left(\sigma^{0, k}, \beta^{0, k}\right)$, it follows that $v\left(\sigma^{0, k}, \beta^{0, k}\right)$ converges to $v\left(\sigma^{0}, \beta^{0}\right)$. We know that

$$
u_{n+1}^{k}=u_{n+1}\left(\mu^{k}, \sigma^{k}, \mu^{k}\right) \leq v\left(\sigma^{0, k}, \beta^{0, k}\right)
$$

for every $k$. Using the fact that $u_{n+1}^{k}$ converges to $\bar{u}_{n+1}$, we may conclude that $v\left(\sigma^{0}, \beta^{0}\right) \geq \bar{u}_{n+1}$.

Finally, we transform $\left(\sigma^{0}, \beta^{0}\right)$ into a sequential equilibrium $(\mu, \sigma, \beta)$ of $\Gamma^{*}$ which gives the information seller an expected payoff of $v\left(\sigma^{0}, \beta^{0}\right)$. The assessment $(\mu, \sigma, \beta)$ is defined in the same way as in the first part of the proof where we constructed a sequential equilibrium $(\mu, \sigma, \beta)$ starting from an a sequential equilibrium $\left(\sigma^{0}, \beta^{0}\right)$ of the game $\Gamma^{0}$.

In order to show sequential rationality of the buyers in $(\mu, \sigma, \beta)$, note that $\mu$ makes the buyers indifferent between all the partitions they can choose. If a buyer buys a partition $P$ with positive probability in $\sigma$, he chooses an optimal vector of contingent actions. The latter is guaranteed by the fact that $\left(\sigma^{0}, \beta^{0}\right)$ is the limit of assessments $\left(\sigma^{0, k}, \beta^{0, k}\right)$ induced by sequential equilibria $\left(\mu^{k}, \sigma^{k}, \beta^{k}\right)$ in $\Gamma^{*}$.

The seller acts sequentially rational since he asks for every partition $P$ the highest price which the corresponding player is wrilling to pay in $(\sigma, \beta)$. Since $(\mu, \sigma, \beta)$ is consistent, we may conclude that it is a sequential equilibrium in $\Gamma^{*}$.

By construction, $u_{n+1}(\mu, \sigma, \beta)=v\left(\sigma^{0}, \beta^{0}\right)$ which implies that $v\left(\sigma^{0}, \beta^{0}\right) \in$ $u_{n+1}(S E)$. We have seen that $v\left(\sigma^{0}, \beta^{0}\right) \geq \bar{u}_{n+1}$ which is the supremum of 
$u_{n+1}(S E)$. Therefore, we may conclude that $v\left(\sigma^{0}, \beta^{0}\right)=\tilde{u}_{n+1} \in u_{n+1}(S E)$, implying that $\bar{u}_{n+1}$ is the maximum of $u_{n+1}(S E)$. Consequently, $(\mu, \sigma, \beta)$ is an optimal sequential equilibrium.

\subsection{Case of full commitment}

The other extreme we want to consider is a situation in which the seller has maximum commitment power. We model this by assuming that the seller can build in a negotiation phase between him and the buyers before the actual game starts. In this negotiation phase, seller and buyers negotiate about the information that will be offered and the price at which it can be purchased. Moreover, the seller is able to charge the buyers certain amounts of money for offering a specific set of partitions against specific prices. For instance, it could be benificial for buyer 1 if the seller does not sell information to buyer 2 , and therefore buyer 1 is prepared to pay a price to the seller for not selling information to buyer 2 . At the end of the negotiation phase, buyers and seller sign a contract which specifies the partitions that will be offered by the seller, the prices of the partitions and the money that has to be paid in advance by the buyers before the actual game starts. This money can be seen as the price that the buyer is willing to pay for the agreed upon contract.

Formally, this case of full commitment is implemented as follows. At the beginning, the seller chooses a negotiation phase, consisting of some arbitrary extensive form game between the seller and the buyers. The negotion phase chosen is revealed to each buyer. Each terminal node of the negotiation phase represents a contract described above, which is observed by all buyers. If a terminal node is reached, the buyers pay the amounts as specified in the contract and a game starts in which exactly the partitions of the contract are offered againgt the agreed upon prices. However, the negotiation phase should be such that a buyer always has the possibility not to pay anything to the seller.

The maximum commitment power of the seller is due to the fact that the set of partitions and the prices of the partitions are fixed once a contract is signed, and therefore the seller can make binding commitments about what information he will offer and against which price. The seller did not have this possibility in the case of no commitment.

Further on in this section, we show that this new extended game always contains a sequential equilibrium and the payoff for the seller obtains a maximum on the set of sequential equilibria. Therefore, we are able to define the information value being the highest payoff that the seller can obtain in 
a sequential equilibrium of the extended game.

Moreover, we show that the seller can obtain the information value by choosing a very simple negotiation phase described below. Finally, we give a characterization of the information value.

\subsubsection{The standard negotiation phase}

Consider the simple negaotiation phase which is decribed by the following rules. At the beginning, the seller charges every buyer $i$ some price $p_{i}$. Each buyer only observes his own price. Thereupon, the buyers decide simultaneously whether or not to pay this price, modeled by assuming that every buyer can say 'yes' or 'no'. Depending on the 'yes-no'-profile of the buyers, the seller chooses a price vector $c=\left(c_{h}(P)\right)_{h, P}$ which specifies a price $c_{h}(P)$ for every partition $P$ offered at some information set $h$. The final contract consists of the prices $p_{i}$ which have to be paid by the buyers and the prices $c_{h}(P)$ charged for the partitions. We call this negotiation phase the standard negotiation phase.

For every price vector $c$, let $\Gamma(c)$ be the game which follows if a contract with price vector $c$ has been signed. In this game, all originally available partitions are offered against the fixed prices in $c$.

A behavior strategy for the seller in the resulting game is a pair $\mu=\left(\mu_{1}, \mu_{2}\right)$ where $\mu_{1}$ is a probability measure on the set of all possible price vectors $\left(p_{i}\right)_{i=1, \ldots, n}$ consisting of prices to be charged to each buyer and $\mu_{2}$ is a function assigning to every possible 'yes-no' profile $v$ of the buyers a probability distribution $\mu_{2}(v)$ on the set of all possible price vectors $c=\left(c_{h}(P)\right)_{h, P}$. We assume that the seller chooses $\left(\mu_{1}, \mu_{2}\right)$ at the beginning of the game. Therefore, sequential rationality leaves him the freedom to choose suboptimal price vectors $c$ after 'yes-no'-profiles which are not realized.

A behavior strategy profile for the buyers is a pair $\sigma=\left(\sigma_{1}, \sigma_{2}\right)$ where $\sigma_{1}$ is a vector which prescribes for every player $i$ a function assigning to every price vector $p_{i}$ a probability distribution $\sigma_{1, i}$ on $\left\{\right.$ yes,no\} and $\sigma_{2}$ is a function assigning to every combination of price vector $p$, 'yes-no' profile and price vector $c$ a behavior strategy profile of the game $\Gamma(c)$.

A belief system is a function $\beta$ assigning to every combination of price vector $p$, 'yes-no'-profile and price vector $c$ a belief system of the subgame $\Gamma(c)$.

\subsubsection{Characterization of information value}

Before we prove the existence of sequential equilibria and give a characteization of the information value, we need some more definitions. 
In every subgame $\Gamma(c)$ following the standard negotiation phase, the set of sequential equilibria $(\sigma(c), \beta(c))$ is called $S E(\Gamma(c))$. Note that $\Gamma(c)$ is a finite extensive form game played only by buyers. Therefore, the existence of sequential equilibria in $\Gamma(c)$ is guaranteed. By

$$
\bar{u}=\sup _{c} \max _{(\sigma(c), \theta(c)) \in S E(\Gamma(c))} \sum_{i=1}^{n+1} u_{i}(\sigma(c), \beta(c))
$$

we denote the highest sum of the payoffs (including the payoff of the seller) which could possibly be achieved in any sequential equilibrium of any subgame $\Gamma(c)$.

For every information buyer $i$ let

$$
\underline{u}_{i}=\inf _{c} \operatorname{in}_{(\sigma(c), \beta(\mathfrak{m}) \in S E(\Gamma(c))\}} u_{i}(\sigma(c), \beta(c))
$$

be the lowest payoff that player $i$ can possibly get in a sequential equilibrium of some subgame $\Gamma(c)$.

Theorem 5.4.1 (a) In the case of full commitment, sequential equilibria always exist and the payoff for the seller reaches a maximum on the set of sequential equilibria.

(b) The information value is equal to $\bar{u}-\sum_{i=1}^{n} \underline{u}_{i}$.

Proof. First, we show that the supremum and the infimum in the definitions of $\vec{u}$ and $\underline{u}_{i}$ are actually a maximum and a minimum.

For every assessment $(\sigma(c), \beta(c))$ of the subgame $\Gamma(c)$, let

$$
u(\sigma(c), \beta(c))=\sum_{i=1}^{n+1} u_{i}(\sigma(c), \beta(c))
$$

Let $\left(c^{k}, \sigma^{k}\left(c^{k}\right), \beta^{k}\left(c^{k}\right)\right)$ be a sequence such that $\left(\sigma^{k}\left(c^{k}\right), \beta^{k}\left(c^{k}\right)\right) \in S E\left(\Gamma\left(c^{k}\right)\right)$ and

$$
\lim _{k \rightarrow \infty} u\left(\sigma^{k}\left(c^{k}\right), \beta^{k}\left(c^{k}\right)\right)=\bar{u}
$$

Since the payoff for the seller and buyers can not increase if the prices in $c$ are too high, we may assume w.l.o.g. that $c^{k}$ is bounded and therefore has a convergent subsequence. W.l.o.g. we assume that $c^{k}$ converges to some $c^{*}$. Since $\left(\sigma^{k}\left(c^{k}\right), \beta^{k}\left(c^{k}\right)\right)$ are assessments of finite extensive form games, we may assume that $\left(\sigma^{k}\left(c^{k}\right), \beta^{k}\left(c^{k}\right)\right)$ converges to an assessment $\left(\sigma\left(c^{*}\right), \beta\left(c^{*}\right)\right)$. As can be seen easily, $\left(\sigma\left(c^{*}\right), \beta\left(c^{*}\right)\right.$ is a sequential equilibrium in $\Gamma\left(c^{*}\right)$. Since $u$ depends continuously on the assessment, we have that.

$$
u\left(\sigma\left(c^{*}\right), \beta\left(c^{*}\right)\right)=\lim _{k \rightarrow \infty} u\left(\sigma^{k}\left(c^{k}\right), \beta^{k}\left(c^{k}\right)\right)=\bar{u},
$$


which implies that the supremum in the definition of $\bar{u}$ is a maximum which is obtained at the sequential equilibrium $\left(\sigma\left(c^{*}\right), \beta\left(c^{*}\right)\right)$ of the subgame $\Gamma\left(c^{*}\right)$. In the same way, we can show that the infimum in the definition of $u_{i}$ is a minimum obtained at a sequential equilibrium $\left(\sigma\left(c^{i}\right), \beta\left(c^{i}\right)\right)$ of some subgame $\Gamma\left(c^{i}\right)$.

Now, we construct a sequential equilibrium which gives the information seller an expected payoff equal to $\bar{u}-\sum_{i=1}^{n} \underline{u}_{i}$.

At the beginning of the game, the seller chooses the standard negotiation phase. In the negotiation phase, seller and buyers play as follows.

For every buyer $i$ let.

$$
p_{i}^{*}=u_{i}\left(\sigma\left(c^{*}\right), \beta\left(c^{*}\right)\right)-\underline{u}_{i} .
$$

The seller puts probability one on the price vector $p^{*}=\left(p_{i}^{*}\right)_{i=1, \ldots, n, n}$. If all buyers say 'yes', he chooses the price vector $c^{*}$. If all players, except player $i$, say 'yes' he 'punishes' player $i$ by choosing $c^{i}$.

On the equilibrium path, the buyers all say 'yes' and play the sequential equilibrium $\left(\sigma\left(c^{*}\right), \beta\left(c^{*}\right)\right)$. If buyer $i$ would deviate by saying ' $n o^{\text {', the buyers }}$ play the sequential equilibrium $\left(\sigma\left(c^{i}\right), \beta\left(c^{i}\right)\right)$ which gives player $i$ the payoff $\underline{u}_{i}$. In all other cases, an arbitrary sequential equilibrium $(\sigma(c), \beta(c))$ of the corresponding subgame $\Gamma(c)$ is played.

The payoff for the seller is equal to

$$
\begin{aligned}
\sum_{i=1}^{n} p_{i}^{*}+u_{n+1}\left(\sigma\left(c^{*}\right), \beta\left(c^{*}\right)\right) & =\sum_{i=1}^{n}\left[u_{i}\left(\sigma\left(c^{*}\right), \beta\left(c^{*}\right)\right)-\underline{u}_{i}\right]+u_{n+1}\left(\sigma\left(c^{*}\right), \beta\left(c^{*}\right)\right) \\
& =\bar{u}-\sum_{i=1}^{n} \underline{u}_{i} .
\end{aligned}
$$

In order to show that this is a sequential equilibrium, it suffices to show that the seller can never obtain a higher payoff by choosing some other negotiation phase, given that the buyers play a sequential equilibrium in the game following this negotiation phase. This result also implies that the payoff $\bar{u}-\sum_{i=1}^{n} \underline{u}_{i}$ is the highest payoff that can be obtained by the seller in a sequential equilibrium and is therefore equal to the information value.

Consider an arbitrary sequential equilibrium. Since the negotiation phase chosen by the seller is observed by all buyers, we may assume w.l.o.g. that the seller chooses a specific negotiation phase with probability one. Consider an arbitrary terminal node of this negotiation phase reached with positive probability, resulting in a contract in which the buyers pay prices $p_{i}$, the set $\mathcal{P}^{*}$ of partitions is offered and every partition $P \in \mathcal{P}^{*}$ is sold at price $\bar{c}_{h}(P)$. Let $\bar{c}$ be the price vector containing the prices $\bar{c}_{h}(P)$ and $\Gamma\left(\mathcal{P}^{*}, \bar{c}\right)$ the subgame in which the collection $\mathcal{P}^{*}$ of partitions is offered at prices in $\bar{c}$. 
Assume that the buyers play the sequential equilibrium $(\bar{\sigma}, \bar{\beta})$ in $\Gamma\left(\mathcal{P}^{*}, \bar{c}\right)$. Let $c$ be the price vector containing prices for all originally available partitions in $\mathcal{P}$ in which partitions in $\mathcal{P}^{*}$ are offered at the same price as in $\bar{c}$ and all other partitions are offered at a very high price, assuring that no buyer will purchase these partitions. Then, the sequential equilibrium $(\bar{\sigma}, \bar{\beta})$ in $\Gamma\left(\mathcal{P}^{*}, \vec{c}\right)$ can be mimicked, in the obvious way, by a sequential equilibrium in the subgame $\Gamma(c)$ following the standard negotiation phase. Therefore, $\sum_{i=1}^{n+1} u_{i}(\bar{\sigma}, \bar{\beta}) \leq \bar{u}$.

Since every buyer $i$ can always guarantee the payoff $\underline{u}_{i}$ in any sequential equilibrium, the price $p_{i}$ can not be higher than $u_{i}(\bar{\sigma}, \bar{\beta})-\underline{u}_{i}$. Therefore, the payoff for the seller after this terminal node of the negotiation phase can not exceed

$$
\begin{aligned}
& \sum_{i=1}^{n}\left[u_{i}(\bar{\sigma}, \bar{\beta})-\underline{u}_{i}\right]+u_{n+1}(\bar{\sigma}, \bar{\beta}) \\
= & \sum_{i=1}^{n+1} u_{i}(\bar{\sigma}, \bar{\beta})-\sum_{i=1}^{n} \underline{u}_{i} \leq \bar{u}-\sum_{i=1}^{n} \underline{u}_{i}
\end{aligned}
$$

which leads to the conclusion that the seller can not gain more than $\bar{u}-$ $\sum_{i=1}^{n} \underline{u}_{i}$ by choosing another negotiation phase. This completes the proof.

\subsection{Standard case}

Next, we discuss the case where the information seller has to choose a price for every partition before the actual extensive form game starts. This case is studied extensively in the previous chapter and is therefore called the standard case. The game induced by this situation is determined by the following rules.

At the beginning of the game, the information seller chooses a price $c_{h}(P)$ for every information set $h$ and every partition $P \in \mathcal{P}_{h}$. The prices, collected in the price vector $c_{n}$ are observed by the information buyers who start playing the following extensive form game afterwards.

At every information set $h$, the corresponding player buys a partition $P \in \mathcal{P}_{h}$, observes that a subset $X \in P$ has been reached and chooses a corresponding action $a_{X} \in A(h)$. Again, the actual partition chosen by the player at $h$ is not revealed to the other players. The expected payoffs are defined in the obvious way.

In contrast with the case of no commitment, the information seller has a certain degree of commitment power since he is able to offer prices at an information set which are not optimal once the information set is reached. 
A behavior strategy for the seller is a probability measure $\mu$ on the set of possible price vectors.

For every price vector $c$, let $\Gamma(c)$ be the subgame of the extended game which starts if $c$ is chosen. A behavior strategy profile for the information buyers is a function $\sigma$ which assigns to every possible price vector $c$ a behavior strategy profile $\sigma(c)$ in the subgame $\Gamma(c)$. Here, $\sigma(c)$ is a vector assigning to every information set $h$ a probability distribution $\sigma_{h}(c)$ on the set $\bar{A}(h)$ of reduced local strategies.

A belief system is a function $\beta$ assigning to every price vector $c$ a belief system $\beta(c)$ in the subgame $\Gamma(c)$. The belief system $\beta(c)$ is a vector which defines for every information set $h$ a probability distribution $\beta_{h}$ on $h$.

The assessment $(\mu, \sigma, \beta)$ is called a sequential equilibrium if $(\sigma(c), \beta(c))$ is consistent and sequentially rational for every $c$ and the information seller maximizes his expected payoff given $\sigma$.

In the previous chapter, it has been shown that the extended game of the standard case always contains a sequential equilibrium and the payoff for the seller reaches a maximum on the set of sequential equilibria. Therefore, the information value for this case is well defined.

\subsection{Comparison of information values}

For $₫$ given base game, we denote the information values of the no commitment case, the standard case and the full commitment case by $V^{n c}, V^{s}$ and $V^{f c}$ respectively.

Theorem 5.6.1 For every base game we have $V^{m c} \leq V^{s} \leq V^{f c}$.

Proof. (a) First, we show that $V^{n c} \leq V^{s}$.

Let $(\mu, \sigma, \beta)$ be an optimal sequential equilibrium of the no commitment case with $u_{n+1}(\mu, \sigma, \beta)=V^{n c}$. First, we construct an assessment $\left(\sigma^{0}, \beta^{0}\right)$ of the game $\Gamma^{0}$ in which all prices are zero. (The game $\Gamma^{0}$ has been introduced in the proof of Theorem 5.3.1.) Here, $\sigma^{0}$ is given by

$$
\sigma_{h}^{0}=\int_{c_{h}} \sigma_{h}\left(c_{h}\right) d \mu_{h}
$$

for every information set $h$ and $\beta^{0}=\beta$. For every $h$ and each partition $P \in \mathcal{P}_{h}$, let $v_{h}\left(P \mid \sigma^{0}, \beta^{0}\right)$ be the reservation value of $P$ as defined in the proof of Theorem 5.3.1. Let $c^{*}=\left(c_{h}^{*}(P)\right)_{h, P}$ be the price vector given by $c_{h}^{*}(P)=v_{h}\left(P \mid \sigma^{0}, \beta^{0}\right)$ for every $h$ and $P$. Hence, $c^{*}$ is the price vector which 
rnakes every buyer indifferent between any partition he can buy, given the assessment $\left(\sigma^{\circ}, \beta^{0}\right)$.

Let $\Gamma\left(c^{*}\right)$ be the subgame in the standard case which follows the price vector $c^{*}$. So, the prices of the partitions in $\mathbb{I}\left(c^{*}\right)$ are fixed according to $c^{*}$. By construction, $\left(\sigma^{0}, \beta^{0}\right)$ is a sequential equilibrium in the subgame $\Gamma\left(c^{*}\right)$. Let $u_{n+1}\left(c^{*}, \sigma^{0}, \beta^{0}\right)$ be the expected payoff for the seller in the subgame $\Gamma\left(c^{*}\right)$ if $\left(\sigma^{0}, \beta^{0}\right)$ is played. So,

$$
u_{n+1}\left(c^{*}, \sigma^{0}, \beta^{0}\right)=\sum_{h} \sum_{P \in \mathcal{P}_{h}} \mathbb{P}_{\sigma^{0}}(h) \sigma_{h}^{0}(P) v_{h}\left(P \mid \sigma^{0}, \beta^{0}\right) .
$$

Since the expected payoff for the seller in $(\mu, \sigma, \beta)$ at an information set $h$ can not be more than $\sum_{P \in \mathcal{P}_{h}} \sigma_{h}^{0}(P) v_{h}\left(P \mid \sigma^{0}, \beta^{0}\right)$ it follows that

$$
V^{n c}=u_{n+1}(\mu, \sigma, \beta) \leq \sum_{h} \sum_{P \in \mathbb{P}_{h}} \mathbb{P}_{\sigma^{0}}(h) \sigma_{h}^{0}(P) v_{h}\left(P \mid \sigma^{0}, \beta^{0}\right)=u_{n+1}\left(c^{*}, \sigma^{0}, \beta^{0}\right) .
$$

In the previous chapter, it has been shown that $V^{s}$ is equal to the maximal expected payoff for the seller that can be achieved in any sequential equilibtium of any subgame $\Gamma(c)$. Since $\left(\sigma^{0}, \beta^{0}\right)$ is a sequential equilibrium of the subgame $\Gamma\left(c^{*}\right)$ which gives the seller an expected payoff greater or equal than $V^{n c}$, it follows that $V^{s} \geq V^{n c}$.

(b) Finally, we prove $V^{s} \leq V^{f o}$.

Let $(\mu, \sigma, \beta)$ be an arbitrary sequential equilibrium of the standard case. Since $V^{f c}=\bar{u}-\sum_{i=1}^{n} \underline{u}_{i}$, it is sufficient to show that $u_{n+1}(\mu, \sigma, \beta) \leq \bar{u}-$ $\sum_{i=1}^{n} \underline{u}_{i}$.

In the previous chapter, it is shown that we can find a new sequential equilibrium of the standard case in which the seller chooses a price vector $c$ with probability one and which gives the seller the expected payoff $u_{n+1}(\mu, \sigma, \beta)$. Therefore, we may assume w.llo.g. that $\mu$ puts probability one on some price vector $c$. With $(\sigma(c), \beta(c))$ being the assessment played in the subgame $\Gamma(c)$
we have

$$
\begin{aligned}
u_{n+1}(\mu, \sigma, \beta) & =u_{n+1}(\sigma(c), \beta(c))=\sum_{i=1}^{n+1} u_{i}(\sigma(c), \beta(c))-\sum_{i=1}^{n} u_{i}(\sigma(c), \beta(c)) \\
& \leq \bar{u}-\sum_{i=1}^{n} u_{i}
\end{aligned}
$$

which completes the proof.

Example 5.1. Consider the following base game. 


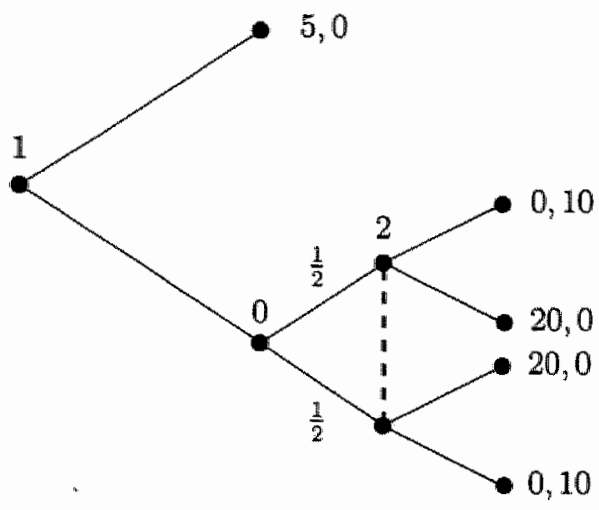

Figure 5.3

Suppose that the seller offers the trivial and the perfect partition at the information set of player 2. We show that $V^{n c}=0, V^{s}=\frac{5}{2}$ and $V^{f c}=10$.

In the no commitment case, sequential rationality of the seller implies that the perfect partition should be offered at price 5 and player 2 should buy the perfect partition with probability one. This follows from the fact that the seller can always guarantee a payoff $5-\varepsilon$ at this information set by offering the perfect partition at price $5-\varepsilon$ which forces player 2 to buy this partition with probability one.

However, if player 2 buys the perfect partition with probability one, he will go up at the upper node and down at the lower node which gives player 1 payoff zero at this information set. Therefore, player 1 will avoid this information set by going up which leaves the information seller with payoff zero. Hence, we may conclude that the information seller can not get more than zero in any sequential equilibrium of the no commitment case and therefore $V^{n c}=0$.

Next, we consider the standard case.

Player 1 is only willing to go down if he gets at least payoff 5 at the information set of player 2. This is only possible if player 2 buys the perfect partition with probability less or equal than $\frac{1}{2}$. Since player 2 pays at most 5 for this partition, the seller can get at most $\frac{5}{2}$ in any sequential equilibrium. In fact, the seller can get $\frac{5}{2}$ in the sequential equilibrium where he offers the perfect partition at price 5, player 1 goes down and player 2 plays $\frac{1}{2}$ (perf,(up,down)) $+\frac{1}{2}$ (triv,up) which implies that $V^{s}=\frac{5}{2}$. Here, (perf, (up,down)) is the reduced local strategy in which player 2 buys the perfect partition, goes up at the upper node and goes down at the lower node 
whereas (triv, up) denotes the reduced local strategy in which he chooses the trivial partition and goes up.

Finally, we focus on the full commitment case.

In order to show that $V^{f c}=10$ we first show that $\vec{u}=15$. This sum of the payoffs can be obtained if the information seller offers the perfect partition at price 100 and therefore forcing player 2 not to buy it, player 1 goes down and player 2 goes up. Clearly, this constitutes a sequential equilibrium for the buyers in the corresponding subgame $\Gamma(c)$. Since the sum of the payoffs can never be more than 15 in a sequential equilibrium of any subgame $\Gamma(c)$, it follows that $\bar{u}=15$.

If the seller offers the trivial and the perfect partition at price zero, player 2 must buy the perfect partition with probability one which leads to payoff zero for player 1 at this information set. Therefore, player 1 goes up and the information buyers get 5 and 0 respectively. As can be seen easily, this is a sequential equilibrium for the information buyers in the subgame $\Gamma(c)$ in which the perfect partition is offered at price zero. Since both players can always guarantee the payoffs 5 and 0 in any sequential equilibrium of any subgame, it follows that $\underline{u}_{1}=5$ and $\underline{u}_{2}=0$. Using Theorem 5.4.1, we may conclude that $V^{f c}=15-(5+0)=10$. 


\section{Chapter 6}

\section{Repeated games with many information mechanisms}

\subsection{Introduction}

This chapter is based on Flesch and Perea y Monsuwé (1997).

Repeated games are a useful tool to model situations in which decision makers face the same mon-cooperative situation a large number of times. In many of these situations, the decision makers are not completely aware of the decisions made by their opponents in the previous periods. Consider, for instance, two countries (potential enemies) which are in the process of developing a nuclear weapon program. In each period, both countries must decide how much weapons to produce or to destroy. The countries are not completely informed about the number of weapons that has been produced by the opponent in the previous periods. However, they can use spy-networks which can secretly inspect the activities of the opponent. In this way, both countries receive partial information about the number of missiles produced by the enemy.

Formally, such situations with imperfect monitoring are modeled by twoplayer repeated games with non-observable actions, of. Lebrer (1989). In these games, both players have an information mechanism which assigns a private signal to each pair of actions chosen in the one-shot game. In the example, the information mechanisms can be indentified with the spynetworks.

For this class of games, Lehrer (1992a) provided a characterization of the lower equilibrium payoffs in terms of the one-shot game. Here, a lower equilibrium denotes a strategy pair in which no player can benefit from switching to another strategy and taking the limit inferior (as the number of stages tends to infinity) of the deviation payoff. A key role in the characterization 
is played by so-called indistinguishable and more informative actions. Two player 1 actions are called indistinguishable if they always induce the same signals for player 2. An action $a_{1}^{\prime}$ is called more informative for player 1 than $a_{1}$ if, whenever player 1 can distinguish between two actions of his opponent by playing $a_{1}$, he can do so by playing $a_{1}^{\prime}$. We say that $a_{1}^{\prime}$ is greater than $a_{1}$ if both actions are indistinguishable and $a_{1}^{\prime}$ is more informative than $a_{1}$. Lehrer shows that the lower equilibrium payoffs for the repeated games are exactly the individually rational payoffs induced by some correlation on the one-shot action pairs, such that no player can benefit from transforming his actions into greater ones. For further reading about repeated games with non-observable actions, we refer to Lehrer $(1989,1990,1991,1992 a, 1992 b)$, Fudenberg and Levine (1989), Fudenberg and Maskin (1986) and Radner (1986).

In this chapter, however, we want to consider the situation in which both players can choose from different information machanisms before the actual game starts. The mechanism that has been chosen is not observed by the opponent. In the example, this means that both countries must decide which spy-network to consult before they start their nuclear program and the countries do not observe the spy-network chosen by the enemy. We assume that the costs for the information mechanisms are fixed and known to both players. Furthermore, the players always have the ability to buy the trivial information mechanism (revealing no information about the opponents actions) at price zero. Therefore, buying the trivial mechanism can be seen as buying no information at all. As a natural condition, we impose that more informative mechanisms (providing more precise information) should be offered at a higher price than less informative ones.

Within this context, we focus on strategies in which both players choose one specific information mechanism with probability one. Such strategies are called simple and lower equilibria consisting of simple strategies are called simple lower equilibria. We provide a characterization of the class of simple lower equilibrium payoffs by means of the one-shot game. In this characterization, the notion of "greaterness between information mechanisms" plays a crucial role. An information mechanism $l_{1}^{\prime}$ is said to be greater than $l_{1}$ if for every action $a_{1}$ there exists an action $a_{1}^{\prime}$ such that playing $a_{1}^{\prime}$ while using $l_{1}^{\prime}$ is indistinguishable from and more informative than playing $a_{1}$ while using $l_{1}$.

Without going into too much detail, the characterization states that the simple lower equilibrium payoffs are exactly the individually rational payoffs generated by some correlation on the one-shot action pairs, such that no player can benefit from switching to a greater information mechanism and subsequently transforming actions into greater ones. Here, it should be 
mentioned that 'greaterness of actions' depends crucially on the information mechanism that is used.

An important part of the proof is to show that deviations to non-greater information mechanisms can always be detected by the opponent by using the so-called information mechanism inspection. This inspection can be seen as test in which a player asks his opponent questions about the signals that he should have received in previous stages. The test is contructed in such a way that, if the opponent switches to a non-greater mechanism, he will fail the test with probability one and can therefore be punished for this deviation. Another interesting situation would be the case where both players are allowed to randomize over the different information mechanisms that will be chosen. However, this situation is much more complex since both players are uncertain about the mechanism that is used by the opponent. Therefore, a player does not know what signals should be received by the opponent and it becomes difficult to check whether the opponent has deviated from the agreed upon strategy or not. This situation is a topic for further research. The outline of the chapter is as follows. In section 2 we introduce the model of repeated games with non-observable actions and fixed information mechanisms and state Lehrer's characterization of the set of lower equilibrium payoffs. Section 3 describes the situation where players can choose from different information mechanisms before playing the repeated game. A characterization of the set of simple lower equilibrium payoffs in this situation is given in section 4 ,

\subsection{Standard model of repeated games}

\subsubsection{Repeated games with non-observable actions}

In this chapter, we consider two-player repeated games in which each player is only partially informed about the opponents actions. Formally, the partial information is modeled by so-called information mechanisms which give both players a private signal at the end of each stage. The signal is dependent on the pair of actions chosen in this stage. Games of this kind are called two-player repeated games with non-observable actions.

Formally, a two-player repeated game with non-observable actions is a triple $\Gamma=\left\langle\Gamma_{N}, l_{1}, l_{2}\right\rangle$ where

(1) $\Gamma_{N}$ is a two-player normal form game with finite action sets $A_{1}, A_{2}$ and payoff-functions $v_{1}, v_{2}$ and

(2) $l_{i}: A_{1} \times A_{2} \rightarrow L_{i}$ is the so-called information mechanism for player $i$ which assigns to every pair of actions a signal in the signals set $L_{i i}$. The 
functions $l_{1}, l_{2}$ should be such that $l_{1}\left(a_{1}, a_{2}\right) \neq l_{1}\left(a_{1}^{s}, a_{2}^{\prime}\right)$ whenever $a_{1} \neq a_{1}^{\prime}$ and $l_{2}\left(a_{1}, a_{2}\right) \neq l_{2}\left(a_{1}^{\prime}, a_{2}^{\prime}\right)$ whenever $a_{2} \neq a_{2}^{\prime}$.

The latter condition on the information mechanisms means that a player always observes his own actions. An information mechanism is called trivial if the corresponding player only observes his own actions. We call the information mechanism perfect if it reveals all the opponents actions.

In the remainder of this chapter, a two-player repeated game with nonobservable actions will simply be called a repeated game.

An n-stage history of player $i$ is a sequence $\left(l_{i}^{1}, l_{i}^{2}, \ldots, l_{i}^{n}\right)$ of player $i$ signals. The set of $n$-stage histories of player $i$ is denoted by $L_{i}^{n}$.

A behavior strategy for player $i$ is a sequence $\sigma_{i}=\left(\sigma_{i}^{n}\right)_{n=1}^{\infty}$ where $\sigma_{i}^{d} \in \Delta\left(A_{i}\right)$ and $\sigma_{i}^{n}: L_{i}^{n-1} \rightarrow \Delta\left(A_{i}\right)$ for $n=2,3, \ldots$. So, in each stage player $i$ prescribes a mixed action after every possible history.

Since a repeated game with non-observable actions is an infinite extensive form game with perfect recall in the sense of Aumann (1964), we can use Aumann's generalization of Kuhn's theorem to show that every mixed strategy has an equivalent behavior strategy. Therefore, we can restrict ourselves to behavior strategies in the remainder of this chapter.

Let $\left(\sigma_{1}, \sigma_{2}\right)$ be a behavior strategy profile (BSP). For every $n$ and every $n$ stage history pair $\left(h_{1}, h_{2}\right), \mathbb{P}_{\sigma_{1}, \sigma_{2}}\left(h_{1}, h_{2}\right)$ denotes the probability that $\left(h_{1}, h_{2}\right)$ will occur. "The expected average payoff for player $i$ at stage $n$ is given by

$$
v_{i}^{n}\left(\sigma_{1}, \sigma_{2}\right):=\frac{1}{n} \sum_{k=1}^{n}\left[\sum_{\left(h_{1}, h_{2}\right) \in L_{1}^{k-1} \times L_{2}^{k-1}} \mathbb{P}_{\sigma_{1}, \sigma_{2}}\left(h_{j}, h_{2}\right) v_{i}\left(\sigma_{1}^{k}\left(h_{1}\right), \sigma_{2}^{k}\left(h_{2}\right)\right)\right] \text {, }
$$

where $L_{i}^{0}$ is some arbitrary single-element set.

We define

$$
\underline{v}_{i}\left(\sigma_{1}, \sigma_{2}\right)=\liminf _{n \rightarrow \infty} v_{i}^{n}\left(\sigma_{1}, \sigma_{2}\right) \text { and } \widetilde{v}_{i}\left(\sigma_{1}, \sigma_{2}\right)=\limsup _{n \rightarrow \infty} v_{i}^{n}\left(\sigma_{1}, \sigma_{2}\right)
$$

The payoffs $v_{i}\left(\sigma_{1}, \sigma_{2}\right)$ and $\bar{v}_{i}\left(\sigma_{1}, \sigma_{2}\right)$ are called lower peyoff and upper payoff respectively.

A $\operatorname{BSP}\left(\sigma_{1}, \sigma_{2}\right)$ is called a lower equilibrium if

(1) $u_{i}\left(\sigma_{1}, \sigma_{2}\right)=\bar{v}_{i}\left(\sigma_{1}, \sigma_{2}\right)$ for $i=1,2$,

(2) $\underline{1}_{1}\left(\sigma_{1}^{\prime}, \sigma_{2}\right) \leq \underline{v}_{1}\left(\sigma_{1}, \sigma_{2}\right)$ for all $\sigma_{1}^{\prime}$ and $\underline{v}_{2}\left(\sigma_{1}, \sigma_{2}^{\prime}\right) \leq \underline{u}_{2}\left(\sigma_{1}, \sigma_{2}\right)$ for all $\sigma_{2}^{\prime}$.

If $\underline{u}_{i}\left(\sigma_{1}, \sigma_{2}\right)=\tilde{v}_{i}\left(\sigma_{1}, \sigma_{2}\right)$, we write $v_{i}\left(\sigma_{1}, \sigma_{2}\right)$ instead of $\underline{v}_{i}\left(\sigma_{1}, \sigma_{2}\right)$. 


\subsubsection{Characterization of lower equilibrium payoffs}

In Lehrer (1992a) a characterization of the lower equilibrium payoffs by means of the one-shot game has been given. In order to state this result we need some more definitions.

First, we consider the case in which both players have a non-trivial information mechanism.

Two player 1 actions $a_{1}^{\prime}, a_{1}$ are called indistinguishoble $\left(a_{1}^{\prime} \sim a_{1}\right)$ if they always induce the same player 2 signals, i.e.

$$
l_{2}\left(a_{1}^{\prime}, a_{2}\right)=l_{2}\left(a_{3}, a_{2}\right)
$$

for all $a_{2}$.

The action $a_{1}^{\prime}$ is called more informative than $a_{1}$ if, whenever player 1 can distinguish between two actions of his opponent by playing $a_{1}$, he can do so by playing $a_{1}^{\prime}$. Formally, this means that

$$
l_{1}\left(a_{1}, a_{2}\right) \neq l_{1}\left(a_{1}, a_{2}^{\prime}\right) \text { implies } l_{1}\left(a_{1}^{\prime}, a_{2}\right) \neq l_{1}\left(a_{1}^{\prime}, a_{2}^{\prime}\right) .
$$

The action $a_{1}^{\prime}$ is called greater than $a_{1}$, denoted by $a_{1}^{\prime} \succ a_{1}$, if $a_{1}^{\prime}$ and $a_{1}$ are indistinguishable and $a_{1}^{\prime}$ is more informative than $a_{1}$.

Let $\mathcal{B}$ be the set of probability distributions on $A_{1} \times A_{2}$ and let $\Psi_{1}$ be the set of functions $\psi_{1}: A_{1} \rightarrow A_{1}$ with $\psi_{1}\left(a_{1}\right) \succ a_{1}$ for all $a_{1} \in A_{1}$. Functions in $\Psi_{1}$ can be seen as mechanisms which transform actions into greater ones. Members of $\mathcal{B}$ are called weight matrices. For every weight matrix $B$ and every $\psi_{1} \in \Psi_{1}$ we define the weight matrix $\psi_{1}(B)$ by

$$
\left(\psi_{1}(B)\right)\left(a_{1}, a_{2}\right)=\sum_{\tilde{a}_{1} \in \psi_{1}^{=1}\left(a_{1}\right)} B\left(\bar{a}_{1}, a_{2}\right)
$$

for all $a_{1}, a_{2}$. Note that, by definition, $\left(\psi_{1}(B)\right)\left(a_{1}, a_{2}\right)=0$ if $\psi_{1}^{-1}\left(a_{1}\right)=\varnothing$.

For a weight matrix $B$, the expected payoff $v_{1}(B)$ is defined in the obvious way.

The set $\mathcal{B}_{1}$ of weight matrices is given by

$$
\mathcal{B}_{1}=\left\{B \in \mathcal{B} \mid v_{1}(B) \geq v_{1}\left(\psi_{1}(B)\right) \text { for all } \psi_{1} \in \Psi_{1}\right\} .
$$

In words, $\mathcal{B}_{1}$ contains those weight matrices for which player 1 can not increase his expected payoff by transforming his actions into greater ones. In the same way, we define $\mathcal{B}_{2}$.

By $d_{1}$ and $d_{2}$ we denote the max-min payoffs for the players in the one-shot game $\Gamma_{N}$ and the set $\left\{\left(v_{1}, v_{2}\right) \mid v_{1} \geq d_{1}\right.$ and $\left.v_{2} \geq d_{2}\right\}$ of individually rational payoffs is denoted by $I R$. 
Theorem 6.2.1 (Lehrer, 1992a) If both players have non-trivial information mechanisms, the set of lower equilibrium payoffs is equal to

$$
v\left(\mathcal{B}_{1}\right) \cap v\left(\mathcal{B}_{2}\right) \cap I R \text {. }
$$

Here, $v\left(\mathcal{B}_{i}\right)$ denotes the set of payoffs $\left(v_{1}, v_{2}\right)$ induced by weight matrices in $\mathcal{B}_{i}$. Lehrer (1992a) defines the sets $\mathcal{B}_{i}$ in another way, but it can be checked easily that his definition is equivalent to ours.

Next, we turn to the case in which one of the players has the trivial information mechanism.

Let $\Psi_{1}^{*}$ be the set of functions $\psi_{1}: A_{1} \rightarrow A_{1}$ with $\psi_{1}\left(a_{1}\right) \sim a_{1}$ for all $a_{1} \in A_{1}$. The set $\mathcal{B}_{1}^{*}$ of weight matrices is given by

$$
\mathcal{B}_{1}^{*}=\left\{B \in \mathcal{B} \mid v_{1}(B) \geq v_{1}\left(\psi_{1}(B)\right) \text { for all } \psi_{1} \in \Psi_{1}^{*}\right\} .
$$

In the same way, we define $\mathcal{B}_{2}^{*}$.

Theorem 6.2.2 (Lehrer, 1992a) If one of the players has the trivial information mechanism, the set of lower equilibrium payoffs is equal to

$$
v\left(\mathcal{B}_{1}^{*}\right) \cap v\left(\mathcal{B}_{2}^{*}\right) \cap I R
$$

\subsection{Many information mechanisms}

Now, we extend the classical model of a repeated game with non-observable actions to a situation in which both players can buy an information mechanism before playing the repeated game. We assume that each player can choose from a given collection of information mechanisms with fixed prices. Moreover, both players do not the information mechanism chosen by the opponent. The game which is obtained in this way is called the extended game. Formally, the extended game is played as follows.

By $\mathcal{L}_{1}$ and $\mathcal{L}_{2}$ we denote the collections of information mechanisms which can be bought by player 1 and 2 respectively. Each information mechanism $L_{i} \in \mathcal{L}_{i}$ can be bought at price $c\left(l_{i}\right)$. We assume that $\mathcal{L}_{1}$ and $\mathcal{L}_{2}$ contain the trivial information mechanism which can be bought at price zero. If the information mechanisms $l_{1}$ and $l_{2}$ are chosen, the players enter the corresponding repeated game $\Gamma\left(l_{1}, l_{2}\right)$. Depending on the information mechanism $l_{i}$ chosen by player $i$, he chooses a behavior strategy $\phi_{i}\left(l_{i}\right)$ in the repeated game which follows. Despite the fact that the player might not know which information mechanism has been chosen by his opponent and therefore be unaware of 
the actual repeated game which is played, he only needs to know his own information mechanism $l_{i}$ to choose a corresponding behavior strategy $\phi_{i}\left(l_{i}\right)$.

A behavior strategy for player $i$ in the extended game is a pair $\sigma_{i}=\left(\tau_{i}, \phi_{i}\right)$ where $\tau_{i}$ is a probability distribution on $\mathcal{L}_{i}$ and $\phi_{i}$ is a function assigning to every information mechanism $l_{i}$ in $\mathcal{L}_{i}$ a corresponding behavior strategy $\phi_{i}\left(l_{i}\right)$ for the repeated game.

A behavior strategy $\sigma_{i}=\left(\tau_{i}, \phi_{i}\right)$ is called simple if $\tau_{i}$ puts probability one on one specific information mechanism $l_{i}$. We denote this strategy by $\left(l_{i}, \phi_{i}\right)$.

Suppose that the simple BSP $\left(\sigma_{1}, \sigma_{2}\right)$ is played in the extended game. The lower (upper) payoff for player $i$ is equal to the difference between the lower (upper) expected payoff in the repeated game and the expected amount to be paid for the information mechanism. We denote the lower and upper expected payoff for player $i$ by $\underline{w}_{i}\left(\sigma_{1_{1}} \sigma_{2}\right)$ and $\bar{w}_{i}\left(\sigma_{1}, \sigma_{2}\right)$ respectively.

The simple BSP $\left(\sigma_{1}, \sigma_{2}\right)$ is called a simple lower equilibrium if

(1) $\underline{w}_{i}\left(\sigma_{1}, \sigma_{2}\right)=\bar{w}_{i}\left(\sigma_{1}, \sigma_{2}\right)$ for $i=1,2$ and

(2) $\underline{w}_{1}\left(\sigma_{1}^{\prime}, \sigma_{2}\right) \leq \underline{w}_{1}\left(\sigma_{1}, \sigma_{2}\right)$ for all $\sigma_{1}^{\prime}$ and $\underline{w}_{2}\left(\sigma_{1}, \sigma_{2}^{\prime}\right) \leq \underline{w}_{2}\left(\sigma_{1}, \sigma_{2}\right)$ for all $\sigma_{2}^{\prime}$.

\subsection{Simple lower equilibria in extended game}

In this section, we provide a characterization of the simple lower equilibrium payoffs of the extended game by means of the one-shot game.

\subsubsection{Characterization of simple lower equilibrium pay- offs}

In the remainder of this chapter, we always refer to the extended game when we talk about strategies and equilibria unless we say otherwise.

By $S L E P\left(l_{1}, l_{2}\right)$ we denote the set of payoffs induced by simple lower equilibria in which $l_{1}$ and $l_{2}$ are chosen with certainty. In order to give a characterization of the set $S L E P\left(l_{1}, l_{2}\right)$ we need some more definitions. In these definitions, $l_{1}$ and $l_{2}$ are fixed. The definitions are illustrated by the following example.

Example 6.1. Let the one-shot game $\Gamma_{N}$ be given by 


\begin{tabular}{|c|c|c|c|c|}
\hline & $X_{1}$ & $X_{2}$ & $X_{3}$ & $X_{4}$ \\
\hline$x_{1}$ & 0,0 & 3,3 & 0,5 & 0,0 \\
\hline$x_{2}$ & 3,3 & 0,0 & 0,5 & 0,0 \\
\hline$x_{3}$ & 5,0 & 0,0 & 0,0 & 0,0 \\
\hline$x_{4}$ & 0,0 & 4,0 & 5,0 & 0,0 \\
\hline$x_{5}$ & 0,0 & 0,0 & 3,3 & 0,0 \\
\hline$x_{6}$ & 0,0 & 0,0 & 0,0 & 0,0 \\
\hline
\end{tabular}

Suppose that the sets of information mechanisms are $\mathcal{L}_{1}=\left\{l_{1}, l_{1}^{\prime}\right\}$ and $\mathcal{L}_{2}=$ $\left\{l_{2}, l_{2}^{\prime}\right\}$, where

$l_{1}=$\begin{tabular}{|l|l|l|l|l|}
\hline & $X_{1}$ & $X_{2}$ & $X_{3}$ & $X_{4}$ \\
\hline$x_{1}$ & $a$ & $a$ & $a$ & $a$ \\
\hline$x_{2}$ & $b$ & $b$ & $b$ & $c$ \\
\hline$x_{3}$ & $d$ & $d$ & $d$ & $d$ \\
\hline$x_{4}$ & $f$ & $f$ & $f$ & $f$ \\
\hline$x_{5}$ & $g$ & $g$ & $g$ & $g$ \\
\hline$x_{6}$ & $h$ & $h$ & $h$ & $h$ \\
\hline
\end{tabular}

$l_{1}^{\prime}=$\begin{tabular}{|l|l|l|l|l|}
\hline & $X_{1}$ & $X_{2}$ & $X_{3}$ & $X_{4}$ \\
\hline$x_{1}$ & $a$ & $a$ & $a$ & $a$ \\
\hline$x_{2}$ & $b$ & $b$ & $b$ & $c$ \\
\hline$x_{3}$ & $d$ & $d$ & $d$ & $e$ \\
\hline$x_{4}$ & $f$ & $f$ & $f$ & $f$ \\
\hline$x_{5}$ & $g$ & $g$ & $g$ & $g$ \\
\hline$x_{6}$ & $h$ & $h$ & $h$ & $h$ \\
\hline
\end{tabular}

$l_{2}=$\begin{tabular}{|l|l|l|l|l|}
\hline & $X_{1}$ & $X_{2}$ & $X_{3}$ & $X_{4}$ \\
\hline$x_{1}$ & $A$ & $B$ & $C$ & $D$ \\
\hline$x_{2}$ & $A$ & $B$ & $C$ & $D$ \\
\hline$x_{3}$ & $A$ & $B$ & $C$ & $D$ \\
\hline$x_{4}$ & $A$ & $B$ & $C$ & $\bar{D}$ \\
\hline$x_{5}$ & $A$ & $B$ & $C$ & $D$ \\
\hline$x_{6}$ & $A$ & $B$ & $C$ & $E$ \\
\hline
\end{tabular}

$l_{2}^{\prime}=$\begin{tabular}{|l|l|l|l|l|}
\hline & $X_{1}$ & $X_{2}$ & $X_{3}$ & $X_{4}$ \\
\hline$x_{1}$ & $A$ & $B$ & $C$ & $D$ \\
\hline$x_{2}$ & $A$ & $B$ & $C$ & $D$ \\
\hline$x_{3}$ & $A$ & $B$ & $C$ & $D$ \\
\hline$x_{4}$ & $A$ & $B$ & $C$ & $D$ \\
\hline$x_{5}$ & $A$ & $B$ & $C$ & $E$ \\
\hline$x_{6}$ & $A$ & $B$ & $C$ & $D$ \\
\hline
\end{tabular}

Here, the symbols $a, b, c, \ldots$ and $A, B, C, \ldots$ denote the signals that player 1 and player 2 receive if an action pair $\left(x_{k}, X_{l}\right)$ is played.

Finally, let the cost functions be given by $c_{1}\left(l_{1}\right)=0, c_{1}\left(l_{1}\right)=1, c_{2}\left(l_{2}\right)=0$ and $c_{2}\left(l_{2}\right)=1$.

Now, let $l_{1}^{\prime}$ be an arbitrary information mechanism. In the following definitions we compare pairs $\left(a_{1} \mid l_{1}\right)$ consisting of an action $a_{1}$ and the information mechanism $l_{1}$ with pairs $\left(a_{1}^{\prime} \mid b_{1}^{\prime}\right)$.

A pair $\left(a_{1}^{s} \mid l_{1}^{\prime}\right)$ is called more informative than $\left(a_{1} \mid l_{1}\right)$, denoted by $\left(a_{1}^{\prime} \mid l_{1}^{\prime}\right) \triangleright$ $\left(a_{1} \mid b_{1}\right)$, if $l_{1}\left(a_{1}, a_{2}\right) \neq l_{1}\left(a_{1}, a_{2}^{\prime}\right)$ implies $\nu_{1}^{\prime}\left(a_{1}^{\prime}, a_{2}\right) \neq l_{1}^{\prime}\left(a_{1}^{\prime}, a_{2}^{\prime}\right)$ for all $a_{2}, a_{2}^{r}$. In words, all actions of player 2 that are distinguished by playing action $a_{1}$ while using information mechanism $l_{1}$ are also distinguished by playing $a_{1}^{\prime}$ while using $l_{1}^{\prime}$. In the example we have, for instance, $\left(x_{3} \mid l_{1}^{\prime}\right) \triangleright\left(x_{2} \mid l_{1}\right)$, but $\left(x_{3} \mid h_{1}\right) \not\left(x_{3} \mid l_{1}^{\prime}\right)$. 
A pair $\left(a_{1}^{\prime} \mid l_{1}^{\prime}\right)$ is called greater than $\left(a_{1} \mid l_{1}\right)$, denoted by $\left(a_{1}^{\prime} \mid l_{1}^{\prime}\right) \succ^{l_{2}}\left(a_{1} \mid l_{1}\right)$, if $a_{1}^{\prime} \sim^{l_{2}} a_{1}$ and $\left(a_{1}^{\prime} \mid l_{1}^{\prime}\right) \triangleright\left(a_{1} \mid l_{1}\right)$. Here, $a_{1}^{\prime} \sim^{l_{2}} a_{1}$ means that $a_{1}^{t}$ and $a_{1}$ are indistinguishable if player 2 uses $l_{2}$. In the example we have $\left(x_{3} \mid l_{1}^{\prime}\right) \succ^{l_{2}}\left(x_{2} \mid l_{1}\right)$ but $\left(x_{2} \mid l_{1}^{\prime}\right) \not x^{i_{2}}\left(x_{6} \mid l_{1}\right)$, because $x_{2} \alpha^{\lambda_{2}} x_{6}$.

An information mechanism $l_{1}^{\prime}$ is called greater than $l_{1}$ with respect to $l_{2}$, denoted by $l_{1}^{\prime} \succ^{l_{2}} l_{1}$, if for every action $a_{1}$ there exists an action $a_{1}^{\prime}$ such that $\left(a_{1}^{\prime} \mid l_{1}^{\prime}\right) \succ^{l_{2}}\left(a_{1} \mid l_{1}\right)$.

In the example, we have that $l_{1}^{\prime} \succ^{l_{2}} l_{1}$ but $l_{2}^{\prime} \not t^{i_{1}} l_{2}$, since $\left(X_{1} \mid l_{2}^{\prime}\right) \neq X^{l_{1}}\left(X_{4} \mid l_{2}\right)$ for all $l$.

Obviously, the perfect information mechanism is greater than any other information mechanism, and all information mechanisms are greater than the trivial one.

As in section 2 , let $\mathcal{B}$ be the set of probability distributions on $A_{1} \times A_{2}$. For an information mechanism $l_{1}^{\prime}$, let $\Psi_{1}\left(l_{1}^{\prime}\right)$ be the set of functions $\psi_{1}: A_{1} \rightarrow A_{1}$ with

$$
\left(\psi_{1}\left(a_{1}\right) \mid l_{1}^{\prime}\right) \succ^{l_{2}}\left(a_{1} \mid l_{1}\right)
$$

for all $a_{1} \in A_{1}$. In words, if player 1 switches from $l_{1}$ to $l_{1}^{\prime}$, then $\Psi_{1}\left(l_{1}^{\prime}\right)$ contains those functions which transform each action into a greater one. Note that $\Psi_{1}\left(l_{1}^{\prime}\right) \neq \emptyset$ if and only if $l_{1}^{\prime} \succ^{l_{2}} l_{1}$.

For a $B \in \mathcal{B}$ and a function $\psi_{1} \in \Psi_{1}\left(l_{1}^{\prime}\right)$, the weight matrix $\psi_{1}(B)$ is defined in the same way as in section 2. Furthermore, we define

$$
\mathcal{B}_{1}\left(l_{1}^{\prime}\right)=\left\{B \in \mathcal{B} \mid v_{\mathbb{I}}(B)-c\left(l_{1}\right) \geq v_{1}\left(\psi_{1}(B)\right)-c\left(l_{1}^{\prime}\right) \text { for all } \psi_{1} \in \Psi_{1}\left(l_{1}^{\prime}\right)\right\} \text {. }
$$

Intuitively, $\mathcal{B}_{1}\left(l_{1}^{\prime}\right)$ contains those weight matrices for which player 1 can not increase his payoff by deviating to information mechanism $l_{1}^{\prime}$ and transforming his actions into greater ones. By construction, $\mathcal{B}_{1}\left(l_{1}^{\prime}\right)=\mathcal{B}$ if $l_{1}^{r} \not \sim^{l_{2}} l_{1}$ since in this case $\Psi_{1}\left(l_{1}^{\prime}\right)=\emptyset$.

In the same way, wre define $\mathcal{B}_{2}\left(\nu_{2}^{\prime}\right)$.

The definitions above enable us to give a characterization of the set $\operatorname{SLEP}\left(l_{1}, l_{2}\right)$, provided that $l_{1}$ and $l_{2}$ are non-trivial.

Theorem 6.4.1 If $l_{1}$ and $l_{2}$ are non-trivial,

$$
\operatorname{SLEP}\left(l_{1}, l_{2}\right)=\left[\left[\bigcap_{l_{1}^{\prime} \in \mathcal{L}_{1}} v\left(\mathcal{B}_{1}\left(l_{1}^{\prime}\right)\right)\right] \cap\left[\bigcap_{l_{2}^{\prime} \in \mathcal{L}_{2}} v\left(\mathcal{B}_{2}\left(l_{2}^{\prime}\right)\right)\right]-\left(c\left(l_{1}\right), c\left(l_{2}\right)\right)\right] \cap I R .
$$

Here, $-\left(c\left(l_{2}\right), c\left(l_{2}\right)\right)$ means that we substract the payoff-pair $\left(c\left(l_{1}\right), c\left(l_{2}\right)\right)$ from every element of the set.

The proof can be found in the remainder of this section. 
Now, we consider the case where either $l_{1}$ or $l_{2}$ is trivial. Let $\Psi_{1}^{*}$ be the set of functions $\psi_{1}: A_{1} \rightarrow A_{1}$ with $\psi_{1}\left(a_{1}\right) \sim^{k_{2}} a_{1}$ for all $a_{1}$. For every information mechanism $l_{1}^{\prime} \in \mathcal{L}_{1}$, we define

$$
\mathcal{B}_{1}^{*}\left(l_{1}^{\prime}\right)=\left\{B \in \mathcal{B} \mid v_{1}(B)-c\left(l_{1}\right) \geq v_{1}\left(\psi_{1}(B)\right)-c\left(l_{1}^{\prime}\right) \text { for all } \psi_{1} \in \Psi_{1}^{*}\right\} \text {. }
$$

In the same way, we define $\mathcal{B}_{2}^{*}\left(l_{2}^{\prime}\right)$.

Theorem 6.4.2 If $l_{1}$ or $l_{2}$ is trivial,

$$
S L E P\left(l_{1}, l_{2}\right)=\left[\left[\bigcap_{l_{1}^{\prime} \in \mathcal{L}_{1}} v\left(\mathcal{B}_{1}^{*}\left(l_{1}^{\prime}\right)\right)\right] \cap\left[\bigcap_{l_{2} \in \mathcal{C}_{2}} v\left(\mathcal{B}_{2}^{*}\left(l_{2}^{\prime}\right)\right)\right]-\left(c\left(l_{1}\right), c\left(l_{2}\right)\right)\right] \cap I R .
$$

\subsubsection{Detectability of deviations to other information mechanisms}

As a first step in the proof of Theorem 6.4.1, we show that a deviation from $l_{1}$ to another information mechanism $l_{1}^{\prime}$ is undetectable if $l_{1}^{\prime} \succ^{l_{2}} l_{1}$ and is detectable otherwise. Before we come to this result, we define what we mean by undetectable and detectable.

By $\Gamma\left(l_{1}, l_{2}\right)$ we denote the repeated game induced by the information mechanisms $l_{1}$ and $l_{2}$. Consider two non-trivial information mechanisms $l_{1}$ and $l_{2}$ which are kept fixed in the remainder of this subsection. We say that a deviation from $l_{1}$ to $l_{1}^{\prime}$ is wndetectable if for all behavior strategy pairs $\left(\sigma_{1}, \sigma_{2}\right)$ in $\Gamma\left(l_{3}, l_{2}\right)$ there exists a behavior strategy $\sigma_{1}^{\prime}$ in $\Gamma\left(l_{1}^{\prime}, l_{2}\right)$ such that $\left(\sigma_{1}^{\prime}, \sigma_{2}\right)$ induces the same probability distribution on the histories of player 2 as $\left(\sigma_{1}, \sigma_{2}\right)$. This means that by choosing $l_{1}$, player 1 can pretend to play any behavior strategy in $\Gamma\left(l_{1}, l_{2}\right)$ without becoming suspicious.

Let $\left(\sigma_{1}, \sigma_{2}\right)$ be a behavior strategy pair in the repeated game $\mathbb{T}\left(l_{1}, l_{2}\right)$. By $I H_{2}\left(\sigma_{1}, \sigma_{2}\right)$ we denote the set of infinite player 2 histories $h_{2}=\left(l_{2}^{1}, l_{2}^{2}, l_{2}^{3}, \ldots\right)$ with $\mathbb{P}_{\sigma_{1}, \sigma_{2}}\left(l_{2}^{n}\right)=0$ for some $n$. So, $I H_{2}\left(\sigma_{1}, \sigma_{2}\right)$ contains those player 2 histories having a finite sub-history which is impossible if $\left(\sigma_{1}, \sigma_{2}\right)$ is played. We say that a deviation from $l_{1}$ to $l_{1}^{\prime}$ is detectable if there exists a strategy pair $\left(\sigma_{1}, \sigma_{2}\right)$ in the repeated game $\Gamma\left(l_{1}, l_{2}\right)$ such that for all behavior strategies $\sigma_{1}^{\prime}$ in $\Gamma\left(l_{1}^{\prime}, l_{2}\right)$ we have

$$
\mathbb{P}_{\sigma_{1}^{\prime}, \sigma_{2}}\left(I H_{2}\left(\sigma_{1}, \sigma_{2}\right)\right)=1
$$

This means that, after choosing $b_{1}^{\prime}$, every strategy of player 1 will induce, with probability 1 , a finite history for player 2 which could not occur if player 1 had chosen $l_{1}$ and played $\sigma_{1}$. Hence, after finitely many stages 
player 2 will become absolutely certain that player 1 has deviated to some other information mechanism. We will say that the deviation can be detected by $\left(\sigma_{1}, \sigma_{2}\right)$.

Now, we describe a strategy pair for finitely many stages in the repeated game that enables both players to detect deviations to non-greater information mechanisms. We call this strategy pair the information mechanism inspection. This inspection consists of a finite number of tests in which a player asks his opponent to report the signals he received at certain previous stages of the game. Formally, the inspection is defined as follows.

Suppose that the set of possible signals for player 1 is $L_{1}=\left\{t_{1}^{1}, \ldots, t t_{1}^{\left|L_{1}\right|}\right\}$. Since $l_{1}$ and $l_{2}$ are non-trivial, there exist actions $a_{1}^{0}, a_{1}^{1} \in A_{1}$ and $\vec{a}_{2} \in A_{2}$ such that $l_{2}\left(a_{1}^{0}, \bar{a}_{2}\right) \neq l_{2}\left(a_{1}^{1}, \bar{a}_{2}\right)$. These actions will be used for communication between the players. For an action $a_{1} \in A_{1}$, the test $T\left\langle a_{1}\right\rangle$ is a strategy pair for $1+\left|L_{1}\right|$ stages defined as follows. At the first stage player 2 plays each of his actions with probability $\frac{1}{\left|A_{2}\right|}$; while at all remaining stages he plays $\bar{a}_{2}$. Player 1 plays action $a_{1}$ at the first stage and afterwards answers which signal he received at the first stage. Player 1 codes his answer in the following way. At the $(1+m)$-th stage he plays action $a_{1}^{1}$ if he received signal $t_{1}^{m}$ at the first stage and he plays action $a_{1}^{0}$ otherwise. Tests $T\left\langle a_{2}\right\rangle$ are defined analogously. The information mechanism inspection is the strategy pair consisting of all possible tests $T\left\langle a_{1}\right\rangle$ and $T\left\langle a_{2}\right\rangle$. Since each test is finite and there are only finitely many tests, it follows that this inspection is finite.

In Example 6.1 we have $l_{2}^{\prime} \not{ }^{l_{1}} l_{2}$, because $\left(X_{i} \mid l_{2}^{\prime}\right) \chi^{l_{1}}\left(X_{4} \mid l_{2}\right)$ for all $l$. Suppose that player 2 deviates from $l_{2}$ to $l_{2}^{\prime}$ and player 1 uses $l_{1}$. At the test $T\left\langle X_{4}\right\rangle$ in the information mechanism inspection, player 2 can not pretend to have chosen $l_{2}$. By using $l_{2}^{\prime}$ player 2 can not distinguish action $x_{6}$ from $x_{1}$ whereas he could do so by using $l_{2}$. Hence, by repeating the test $T\left\langle X_{4}\right\rangle$ an infinite number of times, player 2 will answer incorrectly with probability one.

Lemma 6.4.3 Let $l_{1}, l_{2}$ be non-trivial information mechanisms and $l_{1}^{\prime}$ some other information mechanism.

(a) If $l_{1}^{\prime} \succ^{l_{2}} l_{1}$ then the deviation from $l_{1}$ to $l_{1}^{\prime}$ is undetectable.

(b) If $l_{1}^{\prime} t^{l_{2}} l_{1}$ then the deviation can be detected by using the information mechanism inspection repeatedly.

Proof. (a) Let $l_{1}^{\prime} \succ^{l_{2}} l_{1}$ and $\left(\sigma_{1}, \sigma_{2}\right)$ a behavior strategy pair in $\Gamma\left(l_{1}, l_{2}\right)$. For every $n$, we construct a behavior strategy $\bar{\sigma}_{1, n}$ in $\Gamma\left(l_{1}^{\prime}, l_{2}\right)$ such that $\left(\tilde{\sigma}_{1, n}, \sigma_{2}\right)$ and $\left(\sigma_{1}, \sigma_{2}\right)$ induce the same probability distribution on the $n$-stage histories of player 2 .

Since $l_{1}^{\prime} \succ^{l_{2}} l_{1}$ we can find a function $\psi_{1} \in \Psi_{1}\left(l_{1}^{\prime}\right)$. 
For $n=1$, we define $\vec{\sigma}_{1,1}^{1}\left(a_{1}\right):=\sigma_{1}^{11}\left(\psi_{1}^{-1}\left(a_{1}\right)\right)$ for every $a_{1} \in A_{1}$. At all remaining stages, $\sigma_{1,1}$ is arbitrary. Since $\psi_{1}\left(a_{1}\right) \sim^{l_{2}} a_{1}$ it follows that $\left(\bar{\sigma}_{1,1}, \sigma_{2}\right)$ and $\left(\sigma_{1,}, \sigma_{2}\right)$ induce the same probability distributions on the one-stage histories of playe 2 .

For $n=2$ we construct $\bar{\sigma}_{1,2}$ as follows.

At stage 1 let $\bar{\sigma}_{1,2}$ coincide with $\bar{\sigma}_{1,1}$.

If player 1 observes history $h_{1}^{1}$ at stage 1 , he can compute the conditional probability distribution $\mathbb{P}_{\sigma_{1}, \sigma_{2}}\left(\cdot \mid h_{1}^{\mathbb{I}}\right)$ on the player 1 histories up to stage 1 which would have occurred if he would have played $l_{1}$ and $\sigma_{1}$ instead of $l_{1}^{\prime}$ and $\bar{\sigma}_{1,1}$. The latter is due to the fact that $\left(\psi_{1}\left(a_{1}\right) \mid l_{1}\right)$ is more informative than $\left(a_{1} \mid h_{1}\right)$ for every $a_{1}$. For every $h_{1}^{1}$ we define

$$
\bar{\sigma}_{1,2}^{2}\left(h_{1}^{1}\right)\left(a_{1}\right):=\sum_{\bar{h}_{1}^{1}} \mathbb{P}_{\sigma_{1} \sigma_{2}}\left(\bar{h}_{1}^{1} \mid h_{1}^{1}\right) \cdot \sigma_{1}^{2}\left(\bar{h}_{1}^{1}\right)\left(\psi_{1}^{-1}\left(a_{1}\right)\right)
$$

for all $a_{1}$. At all remaining stages, $\bar{\sigma}_{1,2}$ is arbitrary. By construction, $\left(\bar{\sigma}_{1,2}, \sigma_{2}\right)$ and $\left(\sigma_{1}, \sigma_{2}\right)$ induce the same probability distribution on the two-stage histories of player 2 .

In the same way, we define $\bar{\sigma}_{1, n}$ for $n>2$.

Now, let $\sigma_{1}^{\prime}$ be the behavior strategy which coincides with $\tilde{\sigma}_{1, n}$ at stage $n$. Then, $\left(\sigma_{1}^{\prime}, \sigma_{2}\right)$ induces the same probability distribution on the player 2 histories as $\left(\sigma_{1}, \sigma_{2}\right)$.

(b) Now, let $l_{1}^{\prime} / \succ^{l_{2}} l_{1}$. Then, by definition, there is an action $a_{1}$ for which there is no action $a_{1}^{\prime}$ with $\left(a_{1}^{\prime} \mid l_{1}^{\prime}\right) \succ^{l_{2}}\left(a_{1} \mid l_{1}\right)$. So, player 1 either does not play in an indistinguishable way or he can not distinguish all the actions of player 2 that he could distinguish by playing action $a_{1}$ while using $l_{1}$. In the former case, since each player 2 action occurs with strictly positive probability in the information mechanism inspection, player 2 will, with probability 1 , receive a signal that he should not get if player 1 played $a_{1}$. In the latter case, player 2 will, with probability 1 , receive a wrong answer from player 1 in one of the repetitions of the test $T\left(a_{1}\right)$.

\subsubsection{Proof of characterizations}

Before we prove the characterizations of the simple lower equilibrium payoffs as given in Theorems 6.4.1 and 6.4.2, we need some technical lemmas. The proofs can be found in the appendix. In these lemmas, the information mechanism $l_{1}$ is fixed and $l_{1}^{*}$ is some other information mechanism. The set $\mathcal{B}_{1}\left(l_{1}\right)$ of weight matrices is defined as in subsection 4.1 . 
By $V_{1}\left(l_{1}^{r}\right)$ we define the set of payoff pairs $v$ which can be written as a convex combination

$$
v=\sum_{a_{1}} \alpha^{a_{1}} v\left(a_{1}, p_{2}^{a_{1}}\right)
$$

with

$$
\sum_{a_{1}} \alpha^{a_{1}} v_{1}\left(a_{1}, p_{2}^{a_{1}}\right)-c\left(l_{1}\right) \geq \sum_{a_{1}} \alpha^{a_{1}} v_{1}\left(\psi_{1}\left(a_{1}\right), p_{2}^{a_{1}}\right)-c\left(l_{1}^{\prime}\right)
$$

for all $\psi_{1} \in \Psi_{1}\left(l_{1}^{\prime}\right)$.

Lemma 6.4.4 $v\left(\mathcal{B}_{1}\left(l_{1}^{\prime}\right)\right)=V_{1}\left(l_{1}^{\prime}\right)$.

Proof. (a) " $C$ ". Let $B$ be a weight matrix in $\mathcal{B}\left(l_{1}^{r}\right)$. For every $a_{1} \in A_{1}$ we define

$$
\alpha^{a_{1}}:=\sum_{a_{2} \in A_{2}} B\left(a_{1}, a_{2}\right) \text { and } p_{2}^{a_{1}}\left(a_{2}\right):=\frac{B\left(a_{1}, a_{2}\right)}{\alpha^{a_{1}}}
$$

for all $a_{2}$ if $\alpha^{a_{1}}>0$ and $p_{2}^{a_{2}}$ is arbitrary if $\alpha^{a_{1}}=0$. Then we have $v(B)=\sum_{a_{1}, a_{2}} B\left(a_{1}, a_{2}\right) v\left(a_{1}, a_{2}\right)=\sum_{a_{1}} \alpha^{a_{1}} \sum_{a_{2}} p_{2}^{a_{1}}\left(a_{2}\right) v\left(a_{1}, a_{2}\right)=\sum_{a_{1}} \alpha^{a_{1}} v\left(a_{1}, p_{2}^{a_{1}}\right)$

Suppose that we can find a transformation function $\psi_{1} \in \Psi_{1}\left(l_{1}^{\prime}\right)$ such that

$$
\sum_{a_{1}} \alpha^{a_{1}} v_{1}\left(\psi_{1}\left(a_{1}\right), p_{2}^{a_{1}}\right)-c\left(l_{1}^{\prime}\right)>\sum_{a_{1}} \alpha^{a_{1}} v_{1}\left(a_{1}, p_{2}^{a_{1}}\right)-c\left(l_{1}\right) .
$$

It follows that

$$
\begin{aligned}
v_{1}\left(\psi_{1}(B)\right)-c\left(l_{1}^{\prime}\right) & =\sum_{a_{1}, a_{2}} B\left(\psi_{1}^{-1}\left(a_{1}\right), a_{2}\right) v\left(a_{1}, a_{2}\right)-c\left(l_{1}^{\prime}\right) \\
& =\sum_{a_{1}, a_{2}} B\left(a_{1}, a_{2}\right) v\left(\psi_{1}\left(a_{1}\right), a_{2}\right)-c\left(l_{1}^{\prime}\right) \\
& =\sum_{a_{1}} \alpha^{a_{1}} v\left(\psi_{1}\left(a_{1}\right), p_{2}^{a_{1}}\right)-c\left(l_{1}^{\prime}\right) \\
& >\sum_{a_{1}} \alpha^{a_{1}} v\left(a_{1}, p_{2}^{a_{1}}\right)-c\left(l_{1}\right)=v_{1}(B)-c\left(l_{1}\right),
\end{aligned}
$$

which is a contradiction since $B \in \mathcal{B}_{1}\left(l_{1}^{\prime}\right)$. Therefore, we may conclude that $v(B) \in V_{1}\left(l_{1}^{\prime}\right)$.

(b) " $\supset$ ". Let $v \in V_{1}\left(l_{1}^{l}\right)$, so $v=\sum_{a_{1}} \alpha^{a_{1}} v\left(a_{1}, p_{2}^{a_{1}}\right)$ with

$$
\sum_{a_{1}} \alpha^{a_{1}} v_{1}\left(a_{1}, p_{2}^{a_{1}}\right)-c\left(l_{1}\right) \geq \sum_{a_{1}} \alpha^{a_{1}} v_{1}\left(\psi_{1}\left(a_{1}\right), p_{2}^{w_{1}}\right)-c\left(l_{1}^{\prime}\right)
$$

for all $\psi_{1} \in \Psi_{1}\left(l_{1}^{*}\right)$. 
We define the weight matrix $B$ by

$$
B\left(a_{1}, a_{2}\right)=\alpha^{a_{1}} p_{2}^{a_{1}}\left(a_{2}\right)
$$

for all $a_{1}, a_{2}$. It can be checked easily that $\left.v(B)=\sum_{a_{1}} \alpha^{a_{1}} v\left(a_{1}, p_{2}^{a_{1}}\right)\right)$. It remains to show that $B \in \mathcal{B}_{1}\left(l_{1}^{\prime}\right)$.

Suppose that we can find a $\psi_{1} \in \Psi_{1}\left(l_{1}\right)$ with $v_{1}\left(\psi_{1}(B)\right)-c\left(l_{1}^{\prime}\right)>v_{1}(B)-c\left(l_{1}\right)$. Then, it follows that

$$
\begin{aligned}
\sum_{a_{1}} \alpha^{a_{1}} v_{1}\left(\psi_{1}(\alpha), p_{2}^{a_{1}}\right)-c\left(l_{1}^{v}\right) & =v_{1}\left(\psi_{1}(B)\right)-c\left(l_{1}^{\prime}\right) \\
& >v_{1}(B)-c\left(l_{1}\right) \\
& =\sum_{a_{1}} \alpha^{a_{1}} v_{1}\left(a_{1}, p_{2}^{a_{1}}\right)-c\left(l_{1}\right)
\end{aligned}
$$

which is a contradiction to our assumption that the righthand side of the lemma is satisfied. Therefore, we may conclude that such a $\psi_{1}$ does not exist which implies that $B \in \mathcal{B}_{1}\left(l_{1}^{\prime}\right)$.

It can be checked easily that $v\left(\mathcal{B}_{1}\left(l_{1}^{\prime}\right)\right)$ is a convex and compact set.

Now, suppose that $\left(\sigma_{1}, \sigma_{2}\right)$ is a behavior strategy pair in $\Gamma\left(l_{1}, l_{2}\right)$ inducing a payoff pair $v \notin v\left(\mathcal{B}_{1}\left(l_{1}\right)\right)$. Then, we cen find two disjoint, closed half-spaces $K^{+}$and $K^{-}$such that int $\left(K^{+}\right)$contains $v$ and $K^{-}$contains $v\left(\mathcal{B}_{1}\left(l_{1}^{l}\right)\right)$. Here, $\operatorname{int}\left(K^{+}\right)$denotes the interjor of $K^{+}$.

Lemma 6.4.5 There is a $\gamma>0$ such that $v(B) \in K^{+}$implies that $v_{1}\left(\psi_{1}(B)\right) \geq$ $v_{1}(B)+\gamma$ for some $\psi_{1} \in \Psi_{1}\left(l_{1}^{\prime}\right)$.

Proof. Suppose that such a $\gamma>0$ does not exist. Then, we can find a sequence $B^{k}$ of weight matrices with $v\left(B^{k}\right) \in K^{+}$and $v_{1}\left(\psi_{1}\left(B^{k}\right)\right) \leq v_{1}\left(B^{k}\right)+$ $\frac{1}{k}$ for all $\psi_{1} \in \Psi_{1}\left(l_{1}^{\prime}\right)$. W.l.o.g. we may assume that $B^{k}$ converges to some weight matrix $B$. Since $K^{+}$is closed, it follows that $v(B) \in K^{+}$. On the other hand, we have for all $\psi_{1} \in \Psi_{1}\left(l_{1}^{l}\right)$

$$
v_{1}\left(\psi_{1}(B)\right)=\lim _{k \rightarrow \infty} v_{1}\left(\psi_{1}\left(B^{k}\right)\right) \leq \lim _{k \rightarrow \infty} v_{1}\left(B^{k}\right)+\frac{1}{k}=v_{1}(B)
$$

which implies that $v(B) \in v\left(B_{1}\left(l_{1}^{\prime}\right)\right) \subset K^{-}$. However, this is impossible since $v(B) \in \mathbb{K}^{+}$.

\section{Proof of Theorem 6.4.1.}

(a) " $C$ " Obviously, $S L E P\left(l_{1}, l_{2}\right) \subset I R$ since both players can guarantee their max-min payoff by choosing the trivial information mechanism and playing the max-min action in every stage of the game. 
Now, we show that $\operatorname{SLEP}\left(l_{1}, l_{2}\right) \subset v\left(\mathcal{B}_{1}\left(l_{1}^{\prime}\right)\right)-\left(c\left(l_{1}\right), c\left(l_{2}\right)\right)$ for every $l_{1}^{\prime}$.

Assume by way of contradiction that $\left(\sigma_{1}, \sigma_{2}\right)=\left(\left(l_{1}, \phi_{1}\right),\left(l_{2}, \phi_{2}\right)\right)$ is a lowerequilibrium with payoff $v-\left(c\left(l_{1}\right), c\left(l_{2}\right)\right) \notin v\left(\mathcal{B}_{1}\left(l_{1}^{\prime}\right)\right)-\left(c\left(l_{1}\right), c\left(l_{2}\right)\right)$ for some $l_{1}^{\prime}$, so $v \notin v\left(\mathcal{B}_{1}\left(l_{1}^{\prime}\right)\right)$. For convenience, we write $\phi_{i}$ instead of $\phi_{i}\left(l_{i}\right)$ and $\mathbb{P}$ instead of $\mathbb{P}_{\left(\phi_{1}, \phi_{2}\right)}$. For every $n \in \mathbb{N}$, let $\mathbb{P}^{\text {nn }}\left(a_{1}, a_{2}\right)$ be the probability that the action pair $a_{1}, a_{2}$ is played in stage $n$ and let $B^{n}$ be the weight-matrix given by $B^{n}\left(a_{1}, a_{2}\right)=\mathbb{P}^{n}\left(a_{1}, a_{2}\right)$ for all $a_{1}, a_{2}$. By definition, it holds that

$$
v=\lim _{N \rightarrow \infty} \frac{1}{N} \sum_{n=1}^{N} v\left(B^{n}\right)=\lim _{N \rightarrow \infty} v\left(\frac{1}{N} \sum_{n=1}^{N} B^{n}\right) .
$$

Since $v \notin v\left(\mathcal{B}_{1}\left(u_{1}^{t}\right)\right)$, we can find sets $K^{+}$and $K^{-}$as constructed above. Using the fact that $v \in \operatorname{int}\left(K^{+}\right)$, it follows that $v\left(\frac{1}{N} \sum_{n=1}^{N} B^{n}\right) \in \operatorname{int}\left(K^{+}\right)$ for large $N$. We may assume, w.l.o.g., that $v\left(\frac{1}{N} \sum_{n=1}^{N} B^{n}\right) \in \operatorname{int}\left(K^{+}\right)$for all $N$. By Lemma 6.4.5, there is a $\gamma>0$ such that for every $N$ we can find a $\psi_{1}^{N} \in \Psi_{1}\left(l_{1}^{\prime}\right)$ with

$$
v_{1}\left(\psi_{1}^{N}\left(\frac{1}{N} \sum_{n=1}^{N} B^{n}\right)\right) \geq v_{1}\left(\frac{1}{N} \sum_{n=1}^{N} B^{n}\right)+\gamma
$$

implying that

$$
\frac{1}{N} \sum_{n=1}^{N} v_{1}\left(\psi_{1}^{N}\left(B^{n}\right)\right) \geq \frac{1}{N} \sum_{n=1}^{N} v_{1}\left(B^{n}\right)+\gamma
$$

For every stage $n$, let $\bar{\psi}_{1}^{\text {n. }} \in \Psi_{1}\left(l_{1}^{\prime}\right)$ be such that $v_{1}\left(\bar{\psi}_{1}^{n}\left(B^{n}\right)\right)$ is maximal. With the inequality above, it follows that

$$
\frac{1}{N} \sum_{n=1}^{N} v_{1}\left(\bar{\psi}_{1}^{n}\left(B^{n}\right)\right) \geq \frac{1}{N} \sum_{n=1}^{N} v_{1}\left(\psi_{1}^{N}\left(B^{n}\right)\right) \geq \frac{1}{N} \sum_{n=1}^{N} v_{1}\left(B^{n}\right)+\gamma
$$

for all $N$.

Let $\phi_{1}^{\prime}$ be the behavior strategy which is obtained from $\phi_{1}$ by transforming the actions in stage $n$ according to $\bar{\psi}_{1}^{n}$. The strategy $\phi_{1}^{\prime}$ is constructed in the same way as the strategy $\sigma_{1}^{\prime}$ in the proof of Lemma 6.4.3. Since $\left(\phi_{1}^{\prime}, \phi_{2}\right)$ induces the same probability distributions on the player 2 histories as $\left(\phi_{1}, \phi_{2}\right)$, the deviation from $\phi_{1}$ to $\phi_{1}^{\prime}$ can not be detected. By construction,

$$
v_{1}\left(\phi_{1}^{\prime}, \phi_{2}\right)=\lim _{N \rightarrow \infty} \frac{1}{N} \sum_{n=1}^{N} v_{1}\left(\bar{\psi}_{1}^{n}\left(B^{n}\right)\right) \geq \lim _{N \rightarrow \infty} \frac{1}{N} \sum_{n=1}^{N} v_{1}\left(B^{n}\right)+\gamma=v_{1}+\gamma
$$

which is a contradiction to the fact that $\left(\phi_{1}, \phi_{2}\right)$ is a lower equilibrium.

(b) " $\supset$ " Let $v$ be a payoff in the righthandside. We construct a simple lower equilibrium $\left(\left(l_{1}, \phi_{1}\right),\left(l_{2}, \phi_{2}\right)\right)$ with payoff $v$. The implementation of the 
strategies $\phi_{1}$ and $\phi_{2}$ consists of the following six phases which are repeated infinitely many times.

Phase 1a. Player 1 plays his information mechanism inspection, as described in section 4.2 .

Phase 1b. Same for player 2.

Phase 2a. Choose an information mechanism $l_{1}^{\prime} \in \mathcal{L}_{1}$. By Lemma 6.4.4, $v$ can be written as

$$
v=\sum_{a_{1}} \alpha^{a_{1}} v\left(a_{1}, p_{2}^{a_{1}}\right)
$$

with

$$
\sum_{a_{1}} \alpha^{a_{1}} v_{1}\left(a_{1}, p_{2}^{a_{1}}\right)-c\left(l_{1}\right) \geq \sum_{a_{1}} \alpha^{a_{1}} v_{1}\left(\psi_{1}\left(a_{1}\right), p_{2}^{a_{1}}\right)-c\left(l_{1}^{\prime}\right)
$$

for all $\psi_{1} \in \Psi_{1}\left(l_{1}\right)$.

For every $\varepsilon>0$, let $p_{2}^{a_{1}}(\varepsilon)$ be the unique mixed action closest to $p_{2}^{a_{1}}$ which puts minimal weight $\varepsilon$ on every action.

If the $n$-th stage is reached, let $K$ be the block containing the next $n^{n}$ stages. We divide $K$ into sub-blocks $K^{a_{1}}$ such that the relative length of $K^{\alpha_{1}}$ in $K$ is close to the corresponding coefficient $\alpha^{a_{1}}$. In each stage of block $K^{a_{1}}$, player 1 and 2 play $a_{1}$ and $p_{2}^{a_{1}}(\varepsilon)$ respectively, where $\varepsilon$ has to be chosen small enough. Below, we specify what we mean by "small enough".

On the other hand, the values for $\varepsilon$ have to be chosen in such a way that, starting from an arbitrary stage, the probability that a player 2 action is played in the future, is equal to 1 .

We repeat this process until every information mechanism $l_{1}^{\prime}$ has been chosen.

Phase $2 \mathrm{~b}$. Similar to phase $2 \mathrm{a}$, but now the roles of player 1 and 2 are exchanged.

Phase 3a. Let $S$ be the set of stages containing all repetitions of phase $2 \mathrm{~b}$ up to the current stage. If this is the $n$-th repetition of phase $3 \mathrm{a}$, player 1 asks his opponent the question "Which signal did you receive at the $n$-th stage of $S$ ". Afterwards, player 2 answers the question. The question and answer are implemented in the same way as described in the information mechanism inspection. Since player 2 is supposed to play only pure actions in phase $2 b$, player 1 knows exactly which signal player 2 should have received at the $n$-th stage of $S$.

Phase $3 \mathrm{~b}$. Same as phase 3 a, but now with exchanged roles of player 1 and 2.

At the end of phase $3 \mathrm{~b}$, the players return to phase $1 \mathrm{a}$ and so on. 
If at any moment in the game, a player notices that his opponent has deviated from the prescribed strategy, he will punish him by playing his min-max action for ever.

For a set $M$ of stages, the number

$$
\limsup _{N \rightarrow \infty} \frac{|M \cap\{1, \ldots, N\}|}{N}
$$

is called the upper density of $M$, whereas

$$
\liminf _{N \rightarrow \infty} \frac{|M \cap\{1, \ldots, N\}|}{N}
$$

denotes the lower density of $M$.

By construction, the set of stages belonging to phase $1 \mathrm{a}, 1 \mathrm{~b}, 3 \mathrm{a}$ and $3 \mathrm{~b}$ has upper density zero. Therefore, these phases have no influence on the final payoff.

The lengths of the sub-blocks $K^{a_{1}}, K^{a_{2}}$ in phase $2 \mathrm{a}$ and $2 \mathrm{~b}$ respectively and the value of $\varepsilon$ in the same phases can be chosen in such a way that $v\left(\phi_{1}, \phi_{2}\right)=$ v.

Finally, we show that $\left(\left(l_{1}, \phi_{1}\right),\left(l_{2}, \phi_{2}\right)\right)$ is a lower equilibrium.

Suppose that player 1 would deviate to a strategy $\left(l_{1}^{\prime}, \phi_{1}^{\prime}\right)$.

If $l_{1}^{\prime}$ is not greater than $l_{1}$, then, with probability one, the deviation will be detected in one of the repetitions of phase $\mathrm{lb}$ and player 1 will be punished. So the only possible way for player 1 to improve his payoff is by deviating to an $b_{1}^{\prime \prime} \succ^{l_{2}} l_{1}$.

Look at an arbitrary block $K$ in phase $2 \mathrm{~b}$ whjch corresponds to the information mechanism $l_{1}^{t}$. The length of block $K$ is equal to $n^{n}$, where $n$ is the number of preceding stages. Let $\mathcal{K}$ be the collection of blocks $K$ which correspond to $l_{1}^{\prime}$ and let $\mathcal{K}^{*}$ be the set of final stages of blocks $K \in \mathcal{K}$. Let $x_{1}^{n}$ be the player 1 payoff at stage $n$ when $\left(\phi_{1}^{\prime}, \phi_{2}\right)$ is played. Since in the long run, the average payoff until some stage in $\mathcal{K}^{*}$ is completely determined by the payoffs at stages in $\mathcal{K}$, it follows that

$$
\liminf _{N \in \mathcal{K}^{*}} \frac{1}{N} \sum_{n=1}^{N} x_{1}^{n}=\liminf _{N \in K^{*}} \frac{1}{N} \sum_{n \in \mathcal{K} \cap\{1, \ldots, N\}} x_{1}^{n} .
$$

Since

$$
\underline{v}_{1}\left(\phi_{1}^{\prime}, \phi_{2}\right)=\liminf _{N} v_{1}^{N}\left(\phi_{1}^{\prime}, \phi_{2}\right) \leq \liminf _{N \in \mathcal{K}^{*}} \frac{1}{N} \sum_{n=1}^{N} x_{1}^{n}
$$


it suffices to show that

$$
\liminf _{N \in K^{*}} \frac{1}{N} \sum_{n \in \mathcal{K} \cap\{1, \ldots, N\}} x_{1}^{n} \leq v_{1}\left(\phi_{1}, \phi_{2}\right) .
$$

In other words, it remains to prove that by playing $\phi_{1}^{\prime}$, the average payoff at stages in $\mathcal{K}$ does not exceed $v_{1}\left(\phi_{1}, \phi_{2}\right)$.

Suppose that player 1 deviates in $\mathcal{K}$ by transforming actions infinitely often into distinguishable actions. Then, with probability 1 , player 2 will detect this deviation in one of the repetitions of phase $2 \mathrm{a}$.

If player 1 deviates in phase $2 a$ by transforming actions infinitely often into less informative actions, then, with certainty, this deviation will be detected by player 2 in one of the repetitions of phase $3 \mathrm{~b}$.

So the only possible way for player $\mathbb{1}$ to increase his payoff is by transforming actions infinitely often into greater actions. In the long run, the expected average payoff in stages of $\mathcal{K}$ converges to

$$
v=\sum_{a_{1}} \alpha^{a_{1}} v\left(a_{1}, p_{2}^{a_{1}}\right)
$$

with

$$
\sum_{\alpha_{1}} \alpha^{a_{1}} v_{1}\left(a_{1}, p_{2}^{a_{1}}\right)-c\left(l_{1}\right) \geq \sum_{a_{1}} \alpha^{\alpha_{1}} v_{1}\left(\psi_{1}\left(a_{1}\right), p_{2}^{a_{1}}\right)-c\left(l_{1}^{\prime}\right)
$$

for all $\psi_{1} \in \Psi_{1}\left(l_{1}^{\prime}\right)$. Therefore, player 1 cannot increase his long run average payoff in stages of $\mathcal{K}$ by transforming actions into greater actions, which leads to the conclusion that

$$
\liminf _{N \in \mathcal{K}^{\bullet}} \frac{1}{N} \sum_{n \in \mathcal{K}(1, \ldots, N]} x_{1}^{n} \leq v_{1}\left(\phi_{1}, \phi_{2}\right) .
$$

Since this holds for every $l_{1}^{\prime}$, it follows that $\underline{v}_{1}\left(\phi_{1}^{\prime}, \phi_{2}\right) \leq v_{1}\left(\phi_{1}, \phi_{2}\right)$.

The proof of Theorem 6.4.2 is similar. The only crucial difference is the fact that players are not able not ask questions if one of the players has the trivial information mechanism. Therefore, deviations to less informative actions can not be detected. 


\section{Part III}

\section{Other topics}





\section{Chapter 7}

\section{Player splitting in extensive form games}

\subsection{Introduction}

This chapter is based on Perea y Monsuwé et al. (1996d).

In an extensive form game, each player controls a certain number of information sets. An information set can be interpreted as a situation in which the corresponding player has to reach a decision, based on the information he has at this stage of the game. Formally, the information of a player in such situations is reflected by the nodes which are present in the information set: the player knows that the play of the game has reached one of these nodes, but he does not know which one (if there are at least two nodes in the information set). A pure strategy for a player is simply a plan which prescribes a decision at each of his information sets.

In many practical situations, a player does not reach the decisions himself but uses agents to replace him at certain situations in the game. As a specific example of such a situation, one could think of a firm which delegates certain decisions to departments. In game-theoretic terms, this means that the information sets of a player are divided among agents. Consequently, the extensive form structure of the game (which is obtained by taking all elements of the game except the payoffs) is transformed into a new one in which the player set is the set of agents. Such a transformation of the extensive form structure is called a player splitting. As an extreme example, every player could decide to assign all information sets to different agents. The mixed strategies of this new game are exactly the behavior strategies and the normal form of this new game is called the agent normal form.

In most literature, attention is restricted to a special class of player split 
tings which we call independent player splittings. A player splitting is called independent if in every play of the game, at most one agent of every player appears. In the example this means that, no matter how the game is played, all decisions made for a firm are made by at most one of its departments. So, one could say that the departments of each firm operate independently and therefore there should be no need for communication between the departments. Consequently, such player splittings should not matter too much for the strategic abilities of the firms. The latter is the reason why it is considered a desirable property for a solution concept to be robust against independent player splittings, as is argued by Mertens (1989). This property is formalized in the definition of the well-known player splitting property (Mertens, 1989). We prove that Nash equilibria, perfect equilibria, Kohlberg-Mertens stable sets and Mertens stable sets satisfy the player splitting property. By giving a counterexample, it is shown that the proper equilibrium concept does not satisfy the player splitting property. However, it can be shown that proper equilibria satisfy a weaker version of the player splitting property, which is called the weak player splitting property. Roughly speaking, the latter property says that by applying an independent player splitting, every solution point (set) of the original game induces a solution point (set) of the new game.

The results above can therefore be seen as a justification for the idea that independent player splittings do not really change the strategic abilities of the players.

In the second part of the chapter, we make the step towards dependent (i.e. non-independent) player splittings. Mertens (1989) argues that dependent player splittings, in contrast to independent player splittings, drastically change the strategic situation in the game. We try to support this idea by analyzing the behavior of the above mentioned solution concepts under dependent player splittings. In order to carry out this analysis, we define the notion of invariance under player splittings, which is an extension of the player splitting property to the situation where one also allows for dependent player splittings.

We call a solution concept invariant under a given player splitting if, roughly speaking, the solution of a game does not change by applying this player splitting. We must explain, however, what we mean by 'does not change'. To this purpose, consider an extensive form structure and a player splitting $\pi$ on this structure. A game $\Gamma$ with this structure is transformed by the player splitting into a new game $\Gamma^{\pi}$. Next, we look at a game $\Gamma^{\prime}$ which is equivalent to the game $\Gamma^{\pi}$ in the sense that both games have the same player $i$ payoff at every terminal node which follows a player $i$ information set. We say that the solution 'does not change' if for every such game $\Gamma^{\prime}$ the solution 
coincides with the solution of $\Gamma$. The word "coincides" should not be taken too literally since the games have different strategy spaces. In order to prove that invariance under player splitting is an extension of the player splitting property, we prove that a solution satisfies the player splitting property if and only if it is invariant under every independent player splitting.

We also consider a property which is weaker than invariance under player splittings. A solution is seid to be weakly invariant under a given player splitting if every solution point (set) of the original game $\Gamma$ induces a solution point (set) of $\Gamma^{\prime}$. This definition is an extension of the weak player splitting property.

We come to the conclusion that none of the solution concepts mentioned above is invariant under any dependent player splitting. This result can be seen as a justification for the idea that dependent player splittings drastically change the strategic situation in the game. But more important, we use this insight to give several characterizations of the class of independent player splittings since it turns out to be the largest class of player splittings under which Nash equilibria, perfect equilibria, Kohlberg-Mertens stable sets and Mertens stable sets are invariant. Furthermore, it is the largest class of player splittings under which the proper equilibrium concept is weakly invariant.

Finally, we consider a special class of extensive form structures which we call single appearance structures. A single appearance structure is a structure in which every player appears at most once in every play of the game. Using results of previous sections, we give several characterizations of the class of single appearance structures by means of the invariance of solutions under player splittings.

The outline of the chapter is as follows. In section 2, we present some pre liminaries and give the definition of the player splitting property. In section 3 , we discuss some techical properties of independent player splittings, which prove to be useful in the next section. In section 4 , we investigate the behavior of Nash equilibria, perfect equilibria, proper equilibria, Kohlberg-Mertens stable sets and Mertens stable sets under independent player splittings. Section 5 treats some technical problems which arise if one wants to extend the definition of the player splitting property to dependent player splittings. Afterwards, we give the definition of invariance under player splittings and explain why the definition is chosen in this way. Section 6 is concerned with the behavior of the above mentioned solution concepts under dependent player splittings. Finally, section 7 gives several characterizations of the class of independent player splittings and the class of single appearance structures. 


\subsection{Model and definitions}

In an extensive form game, a pure strategy for player $i$ is a function $m_{i}$ assigning to each information set $h \in H_{i}$ an action in $A(h)$. The set of player $i$ pure strategies will be denoted by $M_{i}$. For a pure strategy profile $m=\left(m_{1}, \ldots, m_{n}\right), v_{i}(m)$ is the payoff to player $i$ when $m$ is played.

The normal form game $\Gamma_{N}=\langle M, v\rangle$, where $M:=\prod_{i} M_{i}$ and $v:=\left(v_{1}, \ldots, v_{n}\right)$ is called the normal form of $\Gamma$.

A mixed strategy for player $i$ is a probability distribution $p_{i}$ on $M_{i}$. For a mixed strategy profile (MSP) $p=\left(p_{1}, \ldots, p_{n}\right), \mathbb{P}_{p}$ denotes the probability distribution on the terminal nodes generated by $p$ and $v_{i}(p)$ is the (expected) payoff to player $i$ induced by $p$.

For a MSP $p$ and a mixed strategy $p_{i}^{\prime}$, the MSP in which player $i$ plays according to $p_{i}^{\prime}$ and all other players play according to $p$ is denoted by $p \backslash p_{i}^{\prime \prime}$. Since the set of mixed strategies profiles depends only on the extensive form structure $\mathcal{S}$, we sometimes talk about mixed strategies in $\mathcal{S}$ instead of mixed strategies in $\Gamma$.

\section{Player splitting property}

Consider an extensive form structure $\mathcal{S}$. A player splitting on $\mathcal{S}$ is a mechanism which divides the information sets of each player between so-called agents. Formally, a player splitting on $\mathcal{S}$ is a function $\pi$ which defines for every player $i$ a partition $\left\{H_{i j} \mid j \in J(i)\right\}$ of the collection $H_{i}$ of information sets controlled by player $i$.

The player splitting $\pi$ induces a new extensive form structure $\mathcal{S}^{\pi}$ in which the player set is given by $N^{r}=\{i j \mid i \in N, j \in J(i)\}$ and every player ij controls the information sets in $H_{i j}$. The players $i j$ are called agents of player i.

For every extensive form game $\Gamma$ with structure $S, \pi$ induces a new game $\Gamma^{\pi}$ with structure $\mathcal{S}^{\pi}$ and payoffs $u_{i j}(z)$ given by $u_{i j}(z):=u_{i}(z)$ for every terminal node $z$.

In $\mathcal{S}^{i}$, pure strategies are usually denoted by $m_{i j}$ and the set of pure strategies of agent $i j$ is given by $M_{i j}$. We use $q_{i j}$ and $q$ to represent mixed strategies and mixed strategy profiles in $\mathcal{S}^{\pi}$ repectively.

A player splitting on $S$ is called independent if on every path in the game tree, at most one agent of every player is present.

As is argued by Mertens (1989), independent player splittings do not really change the strategic abilities of the players. Therefore, it is considered a 
desirable property for a solution to be robust against independent player splittings: a property which is known as the player splatting property and has been introduced by Mertens (1989). In order to recall the definition of the player splitting property, we need some more definitions.

Let $\pi$ be an independent player splitting on $\mathcal{S}$. By $f^{\pi}$, we denote the function which assigns to every MSP $p$ in $\mathcal{S}$ the MSP $q=\left(q_{i j}\right)_{i \in N, j \in J(i)}$ in $\mathcal{S}^{*}$ given by

$$
q_{i j}\left(m_{i j}\right)=\sum_{m_{i:}:\left(m_{i}\right)_{i j}=m_{i j}} p_{i}\left(m_{i}\right)
$$

for every $i \in N, j \in J(i)$ and every $m_{i j} \in M_{i j}$. Here, $\left(m_{i}\right)_{i j}$ denotes the restriction of $m_{i}$ on the information sets in $H_{i j}$.

The function $f^{\pi}$ transforms every mixed strategy $p_{i}$ of $\mathcal{S}$ into an equivalent collection $\left(q_{i j}\right)_{j \in J(i)}$ of mixed strategies of $\mathcal{S}^{\pi}$. The word equivalent means that in every MSP $q^{\prime}$ of $\mathcal{S}^{\pi}$ in which player $i$ plays $\left(q_{i j}\right)_{j \in J(i)}$, we can replace $\left(q_{i j}\right)_{j \in J(i)}$ by $p_{i}$ without changing the probabilities on the terminal nodes. Throughout this chapter, we say that $p$ generates $q$.

Two extensive form games $\Gamma=\langle S, u\rangle$ and $\Gamma^{\prime}=\left\langle\mathcal{S}, u^{\prime}\right\rangle$ are called equivalent if $u_{i}(z)=u_{i}^{\prime}(z)$ whenever player $i$ appears on the path to $z$. Intuitively, this means that, whenever a player has to move, the payoffs for this player are the same in the remainder of the games $\Gamma$ and $\Gamma^{\prime}$.

Let $\varphi$ be a solution assigning to every extensive form game a collection of sets of mixed strategy profiles. We say that the solution $\varphi$ has the player splitting property if for every extensive form game $\Gamma$, every independent player splitting $\pi$ on the structure of $\Gamma$ and every game $\Gamma^{\prime}$ which is equivalent to $\Gamma^{\pi}$ we have that

$$
\varphi\left(\Gamma^{\prime}\right)=\left\{f^{\pi}(S) \mid S \in \varphi(\Gamma)\right\}
$$

and for every $T \in \varphi\left(\Gamma^{\prime}\right)$ it holds that

$$
u\left\{S \in \varphi(\Gamma) \mid f^{\pi}(S)=T^{*}\right\}=\left(f^{\pi}\right)^{-1}(T) .
$$

Here, $S$ and $T$ denote sets of mixed strategy profiles. In words, condition (7.1) means that the solution sets in $\Gamma^{\prime}$ are exactly the images under $f^{\pi}$ of the solution sets in $\Gamma$, whereas condition (7.2) states that the inverse image of a solution set $T$ in $\Gamma^{\prime}$ is the union of solution sets in $\Gamma$ which are mapped onto $T$.

In the case where $\varphi$ is a point valued solution, the two conditions are equivalent to

$$
\varphi(\Gamma)=\left(f^{\pi}\right)^{-1}\left(\varphi\left(\Gamma^{\prime}\right)\right)
$$


We say that $\varphi$ satisfies the weak player splitting property if it holds that

$$
\left\{f^{\pi}(S) \mid S \in \varphi(\Gamma)\right\} \subset \varphi\left(\Gamma^{\prime}\right) \text {. }
$$

Intuitively, this means that $f^{\pi}$ transforms solution setsi in $\Gamma$ into solution sets in $\Gamma^{\prime}$.

The definition of the player splitting property is illustrated by the following diagram.

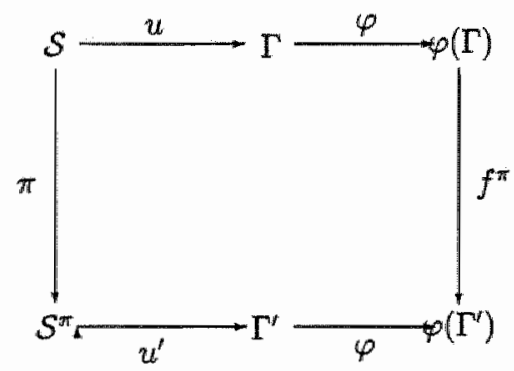

Figure 7.1

\subsection{Technical properties of independent player splittings}

Independent player splittings have the special property that in the resulting game every path crosses at most one agent of every player. In this section, we show that this leads to some very special relationships between the expected payoffs of the original game and the expected payoffs of the new game. Since these results are used repeatedly in section 4 , we dedicate a separate section to these relationships.

Throughout this section, let $\pi$ be an independent player splitting on an extensive form structure $\mathcal{S}, \Gamma=\langle\mathcal{S}, u\rangle$ an extensive form game with structure $S$ and $\Gamma^{\prime \prime}=\left\langle\mathcal{S}^{*}, u^{\prime}\right\rangle$ a game which is equivalent to $\Gamma^{m}$. Let $p$ be a MSP in $\Gamma$ and $q$ a MSP in $\Gamma^{\prime}$ generated by $p$. The expected payoffs in $\Gamma$ and $\Gamma^{\prime}$ are denoted by $v_{i}(p), v_{i j}(q)$ and $v_{i j}(q)$ respectively, whereas the payoffs at the terminal nodes are given by $u_{i}(z), u_{i j}(z)$ and $u_{i j}^{\prime}(z)$ respectively.

For a collection $H^{\prime}$ of information sets, $Z\left(H^{\prime}\right)$ denotes the set of terminal nodes which follow $H^{\prime}$. Since $\pi$ is independent, $Z\left(H_{i}\right)$ is the disjoint union 
of the sets $Z\left(H_{i j}\right)$. Hence, for every MSP $q$ in $\Gamma^{\prime}$ and every agent $i j$,

$$
v_{i j}^{\prime}(q)=\sum_{z \in \mathcal{Z}} \mathbb{P}_{q}(z) u_{i j}^{\prime}(z)=\sum_{z \in \mathcal{Z}\left(H_{i j}\right)} \mathbb{P}_{q}(z) u_{i}(z)+\sum_{z \notin Z\left(H_{i j}\right)} \mathbb{P}_{q}(z) u_{i j}^{\prime}(z)
$$

where the second equality follows from the fact that $u_{i j}^{\prime}(z)=u_{i}(z)$ for all $z \in Z\left(H_{i j}\right)$. This relation plays an important role as well as the following lemma.

Lemma 7.3.1 (a) If

$$
v_{i j}^{i}\left(q \backslash m_{i j}\right)<v_{i j}^{i}\left(q \backslash l_{i j}\right)
$$

then for all pure strategies $m_{i}$ and $l_{i}$ of player $i$ with $\left(m_{i}\right)_{i j}=m_{i j}\left(l_{i}\right)_{i j}=l_{i j}$ and $m_{i}(h)=l_{i}(h)$ for all $h \in H_{i} \backslash H_{i j}$ it holds that

$$
v_{i}\left(p \backslash m_{i}\right)<v_{i}\left(p \backslash l_{i}\right) .
$$

(b) If

$$
v_{i}\left(p \backslash m_{i}\right)<v_{i}\left(p \backslash l_{i}\right)
$$

then there is an agent $i j$ with pure strategies $m_{i j}=\left(m_{i}\right)_{i j}$ and $l_{i j}=\left(l_{i}\right\rangle_{i j}$ such that

$$
v_{i j}^{\prime}\left(q \backslash m_{i j}\right)<v_{i j}^{\prime}\left(q \backslash l_{i j}\right)
$$

Proof. (a) Suppose that $v_{i j}^{\prime}\left(q \backslash m_{i j}\right)<v_{i j}^{\prime}\left(q \backslash l_{i j}\right)$. Let $m_{i}$ and $l_{i}$ be pure strategies of player $i$ with $\left(m_{i}\right)_{i j}=m_{i j},\left(l_{i}\right)_{i j}=l_{i j}$ and $m_{i}(h)=l_{i}(h)$ if $h \in H_{i} \backslash H_{i j}$. Then, we have

$$
\begin{aligned}
v_{i}\left(p \backslash m_{i}\right) & =v_{i j}\left(q \backslash m_{i}\right)=\sum_{z \in Z\left(H_{i j}\right)} \mathbb{P}_{q \backslash m_{i}}(z) u_{i}(z)+\sum_{z \notin Z\left(H_{i j}\right)} \mathbb{P}_{q \backslash m_{i}}(z) u_{i}(z) \\
& =\sum_{z \in Z\left(H_{i j}\right)} \mathbb{P}_{q \backslash m_{i j}}(z) u_{i j}^{\prime}(z)+\sum_{z \notin Z\left(H_{i j}\right)} \mathbb{P}_{q \backslash m_{i}}(z) u_{i}(z) \\
& =v_{i j}^{\prime}\left(q \backslash m_{i j}\right)-\sum_{z \notin Z\left\langle H_{i j}\right)} \mathbb{P}_{q \backslash m_{i j}}(z) u_{i j}^{\prime}(z)+\sum_{z \notin Z\left(H_{i j}\right)} \mathbb{P}_{q \backslash m_{i}}(z) u_{i}(z) \\
& <v_{i j}^{\prime}\left(q \backslash l_{i j}\right)-\sum_{z \notin Z\left(H_{i j}\right)} \mathbb{P}_{q \backslash l_{i j}}(z) u_{i j}^{\prime}(z)+\sum_{z \notin Z\left(H_{i j}\right)} \mathbb{P}_{q \backslash \backslash_{i}}(z) u_{i}(z) \\
& =v_{i}\left(p \backslash l_{i}\right) .
\end{aligned}
$$

Here, the inequality follows from the fact that (1) $v_{i j}^{i}\left(q \backslash m_{i j}\right)<v_{i j}^{i}\left(q \backslash \downarrow_{i j}\right)$ and (2) $\mathbb{P}_{q \backslash m_{i_{j}}}(z)=\mathbb{P}_{q \backslash l_{i_{j}}}(z)$ and $\mathbb{P}_{q \backslash m_{i}}(z)=\mathbb{P}_{q \backslash l_{i}}(z)$ for $z \notin Z\left(H_{i j}\right)$. The last equality is obtained if we substitute $m_{i}$ by $l_{i}$ in the first three equations. 
(b) Suppose that $v_{i}\left(p \backslash m_{i}\right)<v_{i}\left(p \backslash l_{i}\right)$. For a terminal node $z \notin Z\left(H_{i}\right), \mathbb{P}_{q}(z)$ does not depend on player $i$ 's strategy. Hence, for such a $z$ and any pure strategy $r_{i}$ of player $i$ it holds that $\mathbb{P}_{q \nmid r_{i}}(z)=\mathbb{P}_{q}(z)$ leading to

$$
v_{i}\left(p \backslash r_{i}\right)=\sum_{j \in J(i)} \sum_{z \in Z\left\{H_{i j}\right)} \mathbb{P}_{q \backslash r_{i}}(z) u_{i}(z)+\sum_{z \notin Z\left(H_{i}\right)} \mathbb{P}_{q}(z) u_{i}(z) .
$$

Hence, the inequality $v_{i}\left(p \backslash m_{i}\right)<v_{i}\left(p \backslash l_{i}\right)$ implies that

$$
\sum_{j \in J(i)} \sum_{z \in Z\left(H_{i j}\right)} \mathbb{P}_{q \backslash m_{i}}(z) u_{i}(z)<\sum_{j \in J(i)} \sum_{z \in Z\left(H_{i j}\right)} \mathbb{P}_{q \backslash \downarrow_{i}}(z) u_{i}(z)
$$

So we can find an agent $i j$ such that

$$
\sum_{z \in Z\left(H_{i j}\right)} \mathbb{P}_{q \backslash m_{i}}(z) u_{i}(z)<\sum_{z \in \mathcal{Z}\left(H_{i j}\right)} \mathbb{P}_{q \backslash \downarrow_{i}}(z) u_{i}(z) .
$$

By defining $m_{i j}:=\left(m_{i}\right)_{i j}$ and $l_{i j}:=\left(l_{i}\right)_{i j}$ we obtain

$$
\begin{aligned}
. v_{i j}^{\prime}\left(q \backslash m_{i j}\right) & =\sum_{z \in Z\left(H_{i j}\right)} \mathbb{P}_{q \backslash m_{i j}}(z) u_{i}(z)+\sum_{z \notin Z\left(H_{i j}\right)} \mathbb{P}_{q \backslash m_{i}}(z) u_{i j}^{i}(z) \\
& =\sum_{z \in Z\left(H_{i j}\right)} \mathbb{P}_{q \backslash m_{i}}(z) u_{i}(z)+\sum_{z \notin Z\left(H_{i j}\right)} \mathbb{P}_{q}(z) u_{i j}^{\prime}(z) \\
& <\sum_{z \in Z\left(H_{i j}\right)} \mathbb{P}_{q \backslash l_{i}}(z) u_{i}(z)+\sum_{z \notin Z\left(H_{i j}\right)} \mathbb{P}_{q}(z) u_{i j}^{\prime}(z) \\
& =v_{i j}^{\prime}\left(q \backslash l_{i j}\right) .
\end{aligned}
$$

\subsection{Behavior of solutions under independent player splittings}

In this section, we investigate how Nash equilibria, perfect equilibria, proper equilibria, Kohlberg-Mertens stable sets and Mertens stable sets behave under independent player splittings. It turns out that all solution concepts listed above, except the proper equilibria, satisfy the player splitting property.

\subsubsection{Nash equilibria}

The correspondence which assigns to every extensive form game $\Gamma$ the set of Nash equilibria (Nash, 1950) of the normal form is denoted by $N E$. 
Theorem 7.4.1 The Nash equilibrium concept satisfies the player splitting property.

Proof. Let $\Gamma$ be an extensive form game, $\pi$ an independent player splitting on the structure of $\Gamma$ and $\Gamma^{\prime}$ a game which is equivalent to $\Gamma^{\pi}$. The expected payoffs in $\Gamma, \Gamma^{\pi}$ and $\Gamma^{\prime}$ are denoted by $v_{i}(p), v_{i j}(q)$ and $v_{i j}^{\prime}(q)$ respectively. We show that

$$
\left(f^{m}\right)^{-1}\left(N E\left(\Gamma^{\prime}\right)\right)=N E(\Gamma) \text {. }
$$

(a) First we prove that $N E(\Gamma) \subset\left(f^{\pi}\right)^{-1}\left(N E\left(\Gamma^{\prime}\right)\right)$.

Let $p \in N E(\Gamma)$ and let $q=f^{\pi}(p)$. We prove that $q \in N E\left(\Gamma^{\prime}\right)$.

Let $m_{i j}, l_{i j} \in H_{i j}$ and $q_{i j}\left(m_{i j}\right)>0$. We can choose $m_{i}, l_{i}$ with $\left(m_{i}\right)_{i j}=$ $m_{i j},\left(l_{i}\right)_{i j}=l_{i j}$ and $m_{i}(h)=l_{i}(h)$ for all $h \in H_{i} \backslash H_{i j}$. Since $p$ is a Nash equilibrium and $p_{i}\left(m_{i}\right)>0$ we have $v_{i}\left(p \backslash m_{i}\right) \geq v_{i}\left(p \backslash l_{i}\right)$. By Lemma 7.3.1 (a), it follows that $v_{i j}^{\prime}\left(q \backslash m_{i j}\right) \geq v_{i j}^{\prime}\left(q \backslash l_{i j}\right)$. Since this holds for all such $m_{i j}, l_{i j}$ we may conclude that $q$ is a Nash equilibrium in $\Gamma^{\prime}$.

(b) Now, we show that $\left(f^{\pi}\right)^{-1}\left(N E\left(\Gamma^{\prime}\right)\right) \subset N E(\Gamma)$.

Let $p \in\left(f^{\pi}\right)^{-1}\left(N E\left(\Gamma^{\prime}\right)\right)$ and $q=f^{\pi}(p)$. So, by construction, $q \in N E\left(\Gamma^{\prime}\right)$. We prove that $p \in N E(\Gamma)$. Let $m_{i}, l_{i} \in M_{i}$ be such that $v_{i}\left(p \backslash m_{i}\right)<v_{i}\left(p \backslash l_{i}\right)$. Then, by Lemma 7.3 .1 (b), there is an agent $i j$ with pure strategies $m_{i j}=$ $\left(m_{i}\right)_{i j}$ and $l_{i j}=\left(l_{i}\right)_{i j}$ such that $v_{i j}^{\prime}\left(q \backslash m_{i j}\right)<v_{i j}^{\prime}\left(q \backslash l_{i j}\right)$. Since $q$ is a Nash equilibrium in $\Gamma^{\prime}$, it follows that $q_{i j}\left(m_{i j}\right)=0$. Therefore,

$$
0=q_{i j}\left(m_{i j}\right)=\sum_{r_{i}:\left(r_{i}\right)_{i j}=m_{i j}} p_{i}\left(r_{i}\right) \geq p_{i}\left(m_{i}\right)
$$

which implies that $p_{i}\left(m_{i}\right)=0$. Since this holds for every such $m_{i}$, it follows that $p$ is a Nash equilibrium in $\Gamma$.

\subsubsection{Perfect equilibria}

In this chapter, we exploit the following characterization of perfect equilibria (Selten, 1975) for normal form games.

A MSP $p$ is a perfect equilibrium in a normal form game if and only if there is a sequence $\left(p^{k}\right)_{k \in \mathbb{N}}$ of completely mixed MSP's converging to $p$ such that $v_{i}\left(p^{k} \backslash m_{i}\right)<v_{i}\left(p^{k} \backslash l_{i}\right)$ for some $k$ implies $p_{i}\left(m_{i}\right)=0$. By completely mixed, we mean that every pure strategy is played with strictly positive probability. By $P E$, we denote the correspondence which assigns to every extensive form game the set of perfect equilibria of the normal form. This correspondence is therefore different from the original definition of perfect equilibria for extensive form games, which makes use of the agent normal 
form and is given in terms of behavior strategy profiles. Whenever we speak about a perfect equilibrium of the extensive form game $\Gamma$, we mean a perfect equilibrium of the normal form of $\Gamma$.

Before we come to the main result need two technical lemmas. The first lemma can be found in Cook et al. (1986).

Lemma 7.4.2 Let $A$ be a real $m \times n$ matrix, $B:=\{b \mid A x \leq b$ is solvable $\}$ and $\psi(b):=\{x \mid A x \leq b\}$ for every $b \in B$. Then, there is an $L>0$ such that

$$
d_{H}\left(\psi(b), \psi\left(b^{\prime}\right)\right) \leq L \cdot\left\|b-b^{\prime}\right\|
$$

for every $b, b^{\prime} \in B$.

Here, $d_{H}$ denotes the Hausdorff-distance and $\|\cdot\|$ represents the maximum norm.

Lemma 7.4.3 Let $\mathcal{S}$ be an extensive form structure and $\pi$ an independent player splitting on $\mathcal{S}$. Moreover, let $p$ be a $\operatorname{MSP}$ in $\mathcal{S}, q=f^{\pi}(p)$ and $q^{k}$ a sequence of completely mixed MSPS in $\mathcal{S}^{\pi}$ converging to $q$. Then, there is a sequence $p^{k}$ of completely mixed MSPs in $\mathcal{S}$ converging to $p$ with $f^{\pi}\left(p^{k}\right)=q^{k}$ for every $k$.

Proof. Let $\mathcal{M}(\mathcal{S})$ and $\mathcal{M}\left(\mathcal{S}^{\pi}\right)$ be the sets of MSP's in $\mathcal{S}$ and $\mathcal{S}^{\pi}$ respectively. Since the function $f^{\pi}: \mathcal{M}(S) \rightarrow \mathcal{M}\left(\mathcal{S}^{\pi}\right)$ is linear and surjective and the set $\mathcal{M}(\mathcal{S})$ is determined by linear equalities and inequalities, there is a matrix $A$ and an affine function $b$ on $\mathcal{M}\left(\mathcal{S}^{\pi}\right)$ such that

$$
\left(f^{\pi}\right)^{-1}(q)=\{p \mid A p \leq b(q)\}
$$

So, by Lemma 7.42 , we can find a constant $L>0$ such that

$$
d_{B r}\left(\left(f^{\pi}\right)^{-1}(q),\left(f^{\pi}\right)^{-1}\left(q^{\prime}\right)\right) \leq L \cdot\left\|b(q)-b\left(q^{\prime}\right)\right\|
$$

for all $q, q^{\prime}$. Therefore, since $b(q)$ is aftine, there is an $L^{\prime}>0$ with

$$
d_{H}\left(\left(f^{\pi}\right)^{-1}(q),\left(f^{\pi}\right)^{-1}\left(q^{\prime}\right)\right) \leq L^{\prime} \cdot\left\|q-q^{\prime}\right\|
$$

for all $q, q^{\prime}$. Now, let $p$ be a MSP in $\mathcal{S}, q=f^{\pi}(p)$ and $q^{k}$ a sequence of completely mixed MSP's in $\mathcal{S}^{\pi}$ converging to $q$. By the inequality above we can find a sequence $\bar{p}^{k} \in\left(f^{\pi}\right)^{-1}\left(q^{k}\right)$ converging to $p$.

For every $k$, let $\hat{p}^{k}$ be the MSP in $\mathcal{S}$ given by

$$
\hat{p}_{i}^{k}\left(m_{i}\right)=\prod_{i j \in J(i)} q_{i j}^{k}\left(\left(m_{i}\right)_{i j}\right)
$$


for all $i \in N$ and $m_{i} \in M_{i}$. Obviously, $\hat{p}^{k}$ is completely mixed for every $k$. For each $k$ we define $p^{k}$ by

$$
p^{k}=\left(1-\frac{1}{k}\right) \hat{p}^{k}+\frac{1}{k} \hat{p}^{k} .
$$

Since $f^{\pi}\left(\hat{p}^{k}\right)=q^{k}$ and $f^{\pi}\left(\bar{p}^{k}\right)=q^{k}$, it follows by linearity of $f^{\pi}$ that $f^{\pi}\left(p^{k}\right)=$ $q^{k}$. The observation that $p^{k}$ converges to $p$ and $p^{k}$ is cormpletely mixed completes the proof.

Theorem 7.4.4 The perfect equilibrium concept satisfies the player splitting property.

Proof. Let $\Gamma$ be an extensive form game, $\pi$ an independent player splitting on the structure of $\Gamma$ and $\Gamma^{\prime}$ a game which is equivalent to $\Gamma^{*}$. The expected payoffs in $\Gamma, \Gamma^{\pi}$ and $\Gamma^{\prime}$ are denoted by $v_{i}(p), v_{i j}(q)$ and $v_{i j}^{\prime}(q)$ respectively.

(a) First we show that $\left(f^{\pi}\right)^{-1}\left(P E\left(\Gamma^{\prime}\right)\right) \subset P E(\Gamma)$.

Suppose that $p \in\left(f^{\pi}\right)^{-1}\left(P E\left(\Gamma^{\prime}\right)\right)$, which means that $q=f^{\pi}(p)$ is a perfect equilibrium in $\Gamma^{\prime}$. Then there is a sequence $q^{k}$ of completely mixed MSP's converging to $q$ such that $q_{i j}\left(m_{i j}\right)=0$ if $v_{i j}^{\prime}\left(q^{k} \backslash m_{i j}\right)<v_{i j}^{i}\left(q^{k} \backslash l_{i j}\right)$. By Lemma 7.4.3 there is a sequence $p^{k}$ of completely mixed MSP's in $\Gamma$ converging to $p$ with $f^{\pi}\left(p^{k}\right)=q^{k}$.

In order to show that $p$ is perfect, we suppose that $v_{i}\left(p^{k} \backslash m_{i}\right)<v_{i}\left(p^{k} \backslash l_{i}\right)$ for pure strategies $m_{i}$ and $l_{i}$ of player $i$. By Lemma 7.3.1 (b) there is an agent ij with pure strategies $m_{i j}=\left(m_{i}\right)_{i j}$ and $l_{i j}=\left(l_{i}\right)_{i j}$ such that $v_{i j}^{\prime}\left(q^{k} \backslash m_{i j}\right)<$ $v_{i j}^{\prime}\left(q^{k} \backslash l_{i j}\right)$. This implies that $q_{i j}\left(m_{i j}\right)=0$. Since

$$
q_{i j}\left(m_{i j}\right)=\sum_{r_{i}:\left(r_{i} i_{i j}=m_{i j}\right.} p_{i}\left(r_{i}\right)_{n}
$$

it follows that $p_{i}\left(r_{i}\right)=0$ for all $r_{i}$ with $\left(r_{i}\right)_{i j}=m_{i j}$. In particular, $p_{i}\left(m_{i}\right)=0$, which implies that $p$ is a perfect equilibrium for the game $\Gamma$.

(b) Next, we show that $P E(\Gamma) \subset\left(f^{\pi}\right)^{-1}\left(P E\left(\Gamma^{\prime}\right)\right)$.

Let $p \in P E(\Gamma)$. So there is a sequence $p^{k}$ of completely mixed MSP's converging to $p$ such that $p_{i}\left(m_{i}\right)=0$ if $v_{i}\left(p^{k} \backslash m_{i}\right)<v_{i}\left(p^{k} \backslash l_{i}\right)$. We prove that $q=f^{\pi}(p)$ is a perfect equilibrium in $\Gamma^{\prime}$. If $q^{k}$ is the MSP in $\Gamma^{\prime}$ generated by $p^{k}$, then $q^{k}$ is completely mixed and the sequence $q^{k}$ converges to $q$.

In order to show that $q$ is perfect, we suppose that $v_{i j}^{\prime}\left(q^{k} \backslash m_{i j}\right)<v_{i j}^{\prime}\left(q^{k} \backslash l_{i j}\right)$. Let $m_{i}, l_{i}$ be pure strategies with $\left(m_{i}\right)_{i j}=m_{i j},\left(l_{i}\right)_{i j}=l_{i j}$ and $m_{i}(h)=l_{i}(h)$ for all $h \in H_{i} \backslash H_{i j}$. Then, by Lemma 7.3.1 (a), $v_{i}\left(p^{k} \backslash m_{i}\right)<v_{i}\left(p^{k} \backslash l_{i}\right)$ which 
implies that $p_{i}\left(m_{i}\right)=0$. Since this holds for every pure strategy $m_{i}$ with $\left(m_{i}\right)_{i j}=m_{i j}$ it follows that

$$
q_{i j}\left(m_{i j}\right)=\sum_{m_{i:}:} p_{\left(m_{i}\right)_{i j}=m_{i j}} p_{i}\left(m_{i}\right)=0 .
$$

Hence $q$ is a perfect equilibrium for the game $\Gamma^{\prime}$.

\subsubsection{Proper equilibria}

A MSP $p$ is called $\varepsilon$-proper in a normal form game for some $\varepsilon>0$ if it is completely mixed and $v_{i}\left(p \backslash m_{i}\right)<v_{i}\left(p \backslash l_{i}\right)$ implies $p_{i}\left(m_{i}\right) \leq \varepsilon p_{i}\left(l_{i}\right)$. We call $p$ a proper equilibrium (Myerson, 1978) if there is a sequence $\left(\varepsilon^{k}\right)_{k \in \mathbb{A}}$ of strictly positive numbers converging to zero and a sequence $\left(p^{k}\right)_{k \in \mathbb{N}}$ of $\varepsilon^{k}$ proper MSP's converging to $p$. The correspondence which assigns to every extensive form game the set of proper equilibria of the normal form is called $P R$.

Remark 1 The proper equilibrium concept does not satisfy the player splitting property.

Proof. Consider the game $\Gamma$ below and the independent player splitting $\pi$ which divides the information sets of player 1 among the agents $1 \mathrm{a}$ and $1 \mathrm{~b}$ respectively.

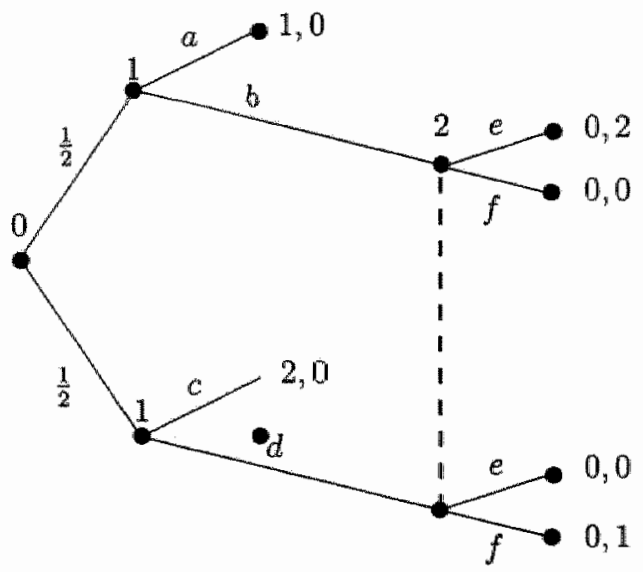

Figure 7.2 
First we prove that the MSP $q=\left(a ; c ; \frac{1}{2} e+\frac{1}{2} f\right)$ is a proper equilibrium in $\Gamma^{\pi}$. As can be seen easily, the MSP

$$
q^{k}=\left(\left(1-\frac{1}{k^{2}}\right) a+\frac{1}{k^{2}} b ;\left(1-\frac{2}{k^{2}}\right) c+\frac{2}{k^{2}} d ; \frac{1}{2} e+\frac{1}{2} f\right)
$$

is $\frac{1}{k}$-proper for the game $\Gamma^{*}$ for large $k$. Furthermore, $q^{k}$ converges to $q$, which implies that $q$ is a proper equilibrium in $\Gamma^{\pi}$.

Next, we show that the unique MSP $p=\left(a c ; \frac{1}{2} e+\frac{1}{2} f\right)$ in $\Gamma$ which induces $q$ is not a proper equilibrium in $\Gamma$. Note that the normal form of $\Gamma$ is given by

\begin{tabular}{|l|l|l|}
\hline & $e$ & $f$ \\
\hline$a c$ & $1 \frac{1}{2}, 0$ & $1 \frac{1}{2}, 0$ \\
\hline$a d$ & $\frac{1}{2}, 0$ & $\frac{1}{2}, \frac{1}{2}$ \\
\hline$b c$ & 1,1 & 1,0 \\
\hline$b d$ & 0,1 & $0, \frac{1}{2}$ \\
\hline
\end{tabular}

Assume that $p$ is a proper equilibrium for the game $\Gamma$. Then there is a sequence $\left(\varepsilon^{k}\right)_{k \in \mathbb{N}}$ of strictly positive numbers converging to zero and a sequence $\left(p^{k}\right)_{k \in \mathbb{N}}$ of $\varepsilon^{k}$-proper MSP's converging to $p$. Since $p_{2}(e)=p_{2}(f)=\frac{1}{2}$, we may assume w.l.o.g. that $v_{2}\left(p^{k} \backslash e\right)=v_{2}\left(p^{k} \backslash f\right)$ for all $k$. Hence,

$$
p_{1}^{k}(b c)+p_{1}^{k}(b d)=\frac{1}{2} p_{1}^{k}(a d)+\frac{1}{2} p_{1}^{k}(b d)
$$

which yields

$$
p_{1}^{k}(b c)=\frac{1}{2} p_{1}^{k}(a d)-\frac{1}{2} p_{1}^{k}(b d) .
$$

Since $v_{1}\left(p^{k} \backslash a d\right)<v_{1}\left(p^{k} \backslash b c\right)$ for all $k$, we get

$$
\frac{1}{2} \varepsilon^{k} p_{1}^{k}(b c) \geq \frac{1}{2} p_{1}^{k}(a d) \geq \frac{1}{2} p_{1}^{k}(a d)-\frac{1}{2} p_{1}^{k}(b d)=p_{1}^{k}(b c)
$$

which is impossible. So we may conclude that $p$ is not a proper equilibrium for the game $\Gamma$. Hence, $p \in\left(f^{\pi}\right)^{-1} P R\left(\Gamma^{\pi}\right)$ but $p \notin P R(\Gamma)$, implying that the proper equilibrium correspondence does not satisfy the player splitting property.

However, we can show that the proper equilibria correspondence satisfies the weak player splitting property. In order to prove this, we need the following lemma. In this lemma, let $\Gamma$ be an extensive form game, $\pi$ an independent player splitting on the structure of $\Gamma$ and $\Gamma^{\prime}$ a game which is equivalent to $\Gamma^{\pi}$.

Lemma 7.4.5 If $p$ is $\varepsilon$-proper in $\Gamma$, then $f^{\pi}(p)$ is $\varepsilon$-proper in $\Gamma^{\prime}$. 
Proof. Let $\varepsilon>0$ and $p$ an $\varepsilon$-proper MSP for the game $\Gamma$. Then, obviously, the MSP $q=f^{\pi}(p)$ is completely mixed since $p$ is completely mixed. In order to show that $q$ is $\varepsilon$-proper for the game $\Gamma^{\prime}$, we prove that $q_{i j}\left(m_{i j}\right) \leq \varepsilon q_{i j}\left(l_{i j}\right)$ if $v_{i j}^{\prime}\left(q \backslash m_{i j}\right)<v_{i j}^{\prime}\left(q \backslash l_{i j}\right)$.

So suppose that $v_{i j}^{\prime}\left(q \backslash m_{i j}\right)<v_{i j}^{\prime}\left(q \backslash l_{i j}\right)$. Then by Lemma 7.3.1 (a), $v_{i}\left(p \backslash m_{i}\right)<$ $v_{i}\left(p \backslash l_{i}\right)$ for all pure strategies $m_{i}$ and $l_{i}$ with $\left(m_{i}\right)_{i j}=m_{i j},\left(l_{i}\right)_{i j}=l_{i j}$ and $m_{i}(h)=l_{i}(h)$ for all $h \in H_{i} \backslash H_{i j}$. Since $p$ is an $\varepsilon$-proper MSP for the game $\Gamma, p_{i}\left(m_{i}\right) \leq \varepsilon p_{i}\left(l_{i}\right)$ for all such pairs $m_{i}, l_{i}$.

For every pure strategy $m_{i}$ with $\left(m_{i}\right)_{i j}=m_{i j}$ we can find a corresponding pure strategy $m_{i} \backslash l_{i j}$ which prescribes $l_{i j}$ at $H_{i j}$ and coincides with $m_{i}$ at $H_{i} \backslash H_{i j}$. Since $p_{i}\left(m_{i}\right) \leq \varepsilon p_{i}\left(m_{i} \backslash l_{i j}\right)$ for such $m_{i}$ we have

$$
\begin{aligned}
q_{i j}\left(m_{i j}\right) & =\sum_{m_{i}:\left(m_{i}\right)_{i j}=m_{i j}} p_{i}\left(m_{i}\right) \leq \sum_{m_{i}:\left(m_{i}\right)_{i j}=m_{i j}} \varepsilon p_{i}\left(m_{i} \backslash l_{i j}\right) \\
& =\sum_{l_{i}:\left(l_{i}\right)_{i j}=l_{i j}} \varepsilon p_{i}\left(l_{i}\right)=\varepsilon q_{i j}\left(l_{i j}\right) .
\end{aligned}
$$

Since this holds for every $m_{i j}$ and $l_{i j}$ with $v_{i j}^{\prime}\left(q \backslash m_{i j}\right)<v_{i j}^{\prime}\left(q \backslash l_{i j}\right)$, it follows that $q$ is $\varepsilon$-proper in the game $\Gamma^{\prime}$.

This result immediately implies the following theorem.

Theorem 7.4.6 The proper equilibria concept satisfies the weak player splitting property.

\subsubsection{Stable sets}

\section{Kohlberg-Mertens stable sets}

In a normal form game $\Gamma$, a set $S$ of MSP's is called a Kohllberg-Mertens stable set (KM-stable set) if it is minimal with respect to the following property: $S$ is closed and for every open set $\mathcal{O}$ containing $S$ there is an $\varepsilon>0$ such that for every strictly positive mistake vector $\eta=\left(\eta_{j}\left(m_{j}\right)\right)_{j \in N_{,} m_{j} \in M_{j} j} \leq \varepsilon$ we have

$$
N E\left(\Gamma^{\eta}\right) \cap \mathcal{O} \neq \emptyset
$$

Here, $\Gamma^{\eta}$ is the restriction of the game $\Gamma$ to mixed strategies $p_{j}$ with $p_{j}\left(m_{j}\right) \geq$ $\eta_{j}\left(m_{j}\right)$ for all $j$ and all $m_{j} \in M_{j}$. The game $\Gamma^{\eta}$ is called a perturbed game. Let $\mathcal{K M}$ be the correspondence which assigns to every extensive form game the collection of KM-stable sets of the normal form. In order to prove the following theorem, we need the definition of invariant solutions which can be found in Mertens (1987), Theorem 2 (b). ${ }^{1}$

\footnotetext{
${ }^{T}$ Mertens did not use the word invariance in this paper.
} 
A normal form solution $\varphi$ is called invariant if for every two games $\Gamma, \Gamma^{*}$ with the same player set and every linear, payoff preserving function $f$ mapping the strategy space of $\Gamma$ onto the strategy space of $\Gamma^{*}$ we have

$$
\varphi\left(\Gamma^{*}\right)=\{f(S) \mid S \in \varphi(\Gamma)\}
$$

and for every $T \in \varphi\left(\Gamma^{*}\right)$ it holds that

$$
\text { - } \cup\{S \in \varphi(\Gamma) \mid f(S)=T\}=f^{-1}(T) .
$$

Theorem 7.4.7 The KM-stability concept satisfies the player splitting property.

Proof. Let $\Gamma$ be an extensive form game, $\pi$ an independent player splitting on the structure of $\Gamma$ and $\Gamma^{\prime}$ a game which is equivalent to $\Gamma^{*}$. Let $\Gamma^{*}$ be the normal form game with the same player set as $\Gamma$ in which the strategy space of player $i$ is the product of the mixed strategy spaces of the corresponding agents $i j$. Let $f$ be the payoff preserving, linear function from the strategy space of $\Gamma$ onto the strategy space of $\Gamma^{*}$ which is obtained by taking the marginals. Since it is shown in Mertens (1987), section 4.2.5 that $\mathcal{K M}$ is invariant, it follows that the Kohlberg-Mertens stable sets of $\Gamma$ and $\Gamma^{*}$ satisfy equations (7.4) and (7.5).

It remains to show that the KM-stable sets of $\Gamma^{*}$ and $\Gamma^{*}$ are the same. However, this follows from the fact that the perturbed games of $\Gamma^{*}$ and $\Gamma^{\prime}$ are the same as is shown in Mertens (1989), proof of Theorem 4. Consequently, the KM-stable sets of $\Gamma_{\text {f }}$ and $\Gamma^{\prime}$ satisfy equations (7.1) and (7.2).

\section{Mertens stable sets}

It would require too much space to give an exact definition of Mertens stable sets (Mertens, 1989). Therefore, we restrict ourselves to stating a geometric definition of Mertens stable sets (M-stable sets), whïch Mertens (1989) describes as follows:

"Finally in (E) we show how the most encompassing definitionthat involving the union over all possible coefficient modules- has a direct geometrical definition (...) The geometric definition involves an essentiality condition- that the projection should not be null homotopic- and a dimensional restriction- that this should remain true when restricted to some subset of the equilibrium graph of the same dimension as the space of perturbed gannes."

Mertens (1989) showed that the Mertens stability concept has the player splitting property. 


\subsection{Invariance under player splittings}

The player splitting property can be viewed as a tool which is used to investigate the behavior of solutions under independent player splittings. Among the solution concepts considered, all but one are robust against independent player splittings. This result supports the idea, stated by Mertens (1989), that independent player splittings do not really change the strategic abilities of the players. On the other hand, Mertens argues that dependent player splittings do change the strategic situation of the game. A natural question which arises is whether this statement can be supported by the behavior of solution concepts under dependent player splittings.

In order to answer this question, we introduce a definition of invariance of solutions under player splittings. This definition mainly follows the idea of the player splitting property: the solutions should not change by applying the player splitting. In the next section, we show that none of the solution concepts above is invariant under any dependent player splitting. Consequently, the class of independent player splittings can be regarded as the largest class of player splittings leaving the strategic situation of the game unchanged.

\section{Technical problems}

However, there are two major technical problems which complicates our attempt to give a suitable definition of invariance under player splitings. In order to compare the solutions of the original game $\Gamma$ and the new game $\Gamma^{\prime \prime}$ we need a surjective function which maps every mixed strategy in $\Gamma$ to an equivalent collection of mixed strategies in $\Gamma^{\prime}$. The first problem is due to the fact that, in contrast with independent player splittings, we are not able to construct such a surjective function if the player splitting is dependent, except for some special cases. If, for instance, the player splitting is maximal, meaning that it assigns each information set to a different agent, we can apply the function used in Kuhn (1953) which transforms every mixed strategy into a equivallent collection of behavior strategies. However, we are not able to generalize this transformation if the player splitting is dependent and not maximal, as is the case in the following example. 


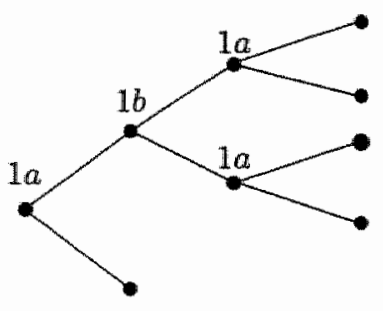

Figure 7.3

Here, the information sets all belong to player 1 in the original game and are distributed among the agents $1 a$ and $1 b$ under the player splitting. It is not clear how we should construct a surjective function mapping mixed strategies of the original game to equivalent collections of mixed strategies of the new game.

In order to overcome this difficulty, we tried to define invariance under player splittings in such a way that the solutions should not change under every such surjective function, without stating explicitly how these functions should look like. Unfortunately, this gives rise to a second problem.

If we restrict ourselves to independent player splittings, there is in general more than one way to construct this surjective function. Consider, for example, the following game.

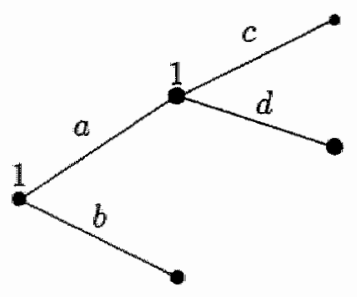

Figure 7.4

Let $\pi$ be the trivial player splitting which leaves the extensive form structure the same. Then, one could choose a function $f$ which transforms the mixed strategy $b c$ into the equivalent mixed strategy $b d$. However, this function is different from the function $f^{\pi}$ in the definition of the player splitting property. As a consequence, the property that the solution should not change under every independent player splitting and every surjective function mapping mixed strategies into equivalent ones is in general strictly stronger than the 
player splitting property, since the latter only requires that the solutions should not change under one such surjective function, namely the function $f^{\pi}$. Since we want invariance under independent player splittings to be equivalent to the player splitting property, we put an extra restriction on the class of surjective functions $f$ which can be applied. This extra restriction says that the function $f$ should transform mixed strategies not only into equivalent but into perfectly equivalent collections of mixed strategies, a term which is defined below. The extra restriction guarantees that the only function which can be chosen at independent player splittings is the function $f^{\pi}$. As a consequence, a solution is invariant under all independent player splittings if and only if it satisfies the player splitting property. Therefore, invariance under player splittings is an extension of the player splitting property.

\section{Definition of invariance under player splittings}

Let $\mathcal{S}$ be an extensive form structure and $\pi$ a player splitting on $\mathcal{S}$. A mixed strategy $p_{i}$ in $\mathcal{S}$ is said to be perfectly equivalent to a collection. $\left(q_{i j}\right)_{j \in J(i)}$ of mixed strategies in $\mathcal{S}^{\pi}$ if there is a sequence $\left(p_{i}^{k}\right)_{k \in \mathbb{N}}$ of completely mixed strategies converging to $p_{i}$ and a sequence $\left.\left(\left(q_{i j}^{k}\right)_{j \in J(i)}\right)\right)_{k \in \mathbb{N}}$ converging to $\left(q_{i j}\right)_{j \in J(i)}$ such that $p_{i}^{k}$ is equivalent to $\left(q_{i j}^{k}\right)_{j \in J(i)}$ for every $k$.

In words, this means that by perturbing $p_{i}$ a bit, we can always find an equivalent collection of mixed strategies in the neighborhood of $\left(q_{i j}\right)_{j \in J(i)}$. Obviously, perfect equivalence always implies equivalence. In the example of figure $4, b c$ is equivalent to $b d$ but they are not perfectly equivalent.

Let $\mathcal{S}$ be an extensive form structure and $\pi$ a player splitting on $\mathcal{S}$. We say that the solution $\varphi$ is invariant under $\pi$ if for every extensive form game $\Gamma$ with structure $\mathcal{S}$, every game $\Gamma^{\prime}$ which is equivalent to $\Gamma^{\pi}$ and every surjective function $f$ which maps mixed strategies in $\mathcal{S}$ to perfectly equivalent collections of mixed strategies in $\mathcal{S}^{\pi}$ we have

$$
\varphi\left(\Gamma^{\prime}\right)=\{f(S) \mid S \in \varphi(\Gamma)\}
$$

and for every $T^{\prime \prime} \in \varphi\left(\Gamma^{\prime}\right)$ it holds that

$$
\cup\{S \in \varphi(\Gamma) \mid f(S)=T\}=f^{-1}(T)
$$

If the solution $\varphi$ is a point valued solution, both conditions are equivalent to

$$
\varphi(\Gamma)=f^{-1}\left(\varphi\left(\Gamma^{\prime}\right)\right)
$$


If it holds that

$$
\{f(S) \mid S \in \varphi(\Gamma)\} \subset \varphi\left(\Gamma^{\prime}\right)
$$

we say that $\varphi$ is weakly invariant under $\pi$.

In the remainder of this chapter, functions $f$ with the properties listed above are called transformation functions.

\section{Relation with player splitting property}

We show that 'invariance under every independent player splitting' is equivalent to the player splitting property. In order to see this, consider an extensive form structure $\mathcal{S}$ and an independent player splitting $\pi$ on $\mathcal{S}$. Every completely mixed strategy $p_{i}$ in $\mathcal{S}$ has only one equivalent collection of mixed strategies in $\mathcal{S}^{\pi}$, mamely $f^{\pi}\left(p_{i}\right)$. Since the function $f^{\pi}$ is continuous, it follows that for each mixed strategy $p_{i}$ in $\mathcal{S}, f^{\pi}\left(p_{i}\right)$ is the only perfectly equivalent collection of mixed strategies in $\mathcal{S}^{\pi}$. Therefore, the only possible transformation function is the function $f^{\pi}$. By comparing the definitions of invariance under player splittings and the player splitting property, we obtain the following lemma.

Lemma 7.5.1 A solution is invariant under every independent player splitting on every extensive form structure if and only if it satisfies the player splitting property.

In view of the lemma above, invariance under player splittings is an extension of the player splitting property. In a similar way, we can show that the solution is weakly invariant under every independent player splitting on every extensive form structure if and only if the solution satisfies the weak player splitting property.

\subsection{Behavior of solutions under dependent player splittings}

We show that none of the solution concepts considered above is invariant under any dependent player splitting. 
Lemma 7.6.1 The Nash equilibrium concept is not invariant under any dependent player splitting.

Proof. Let $\mathcal{S}$ be an extensive form structure and $\pi$ a dependent player splitting on $\mathcal{S}$. Then there are two different agents $i j$ and $i k$ in $\mathcal{S}^{\pi}$ which appear both in a certain play of the game. Formally, this means that we can find a path from the root to $Z$ containing two nodes, say $x \in h$ and $x^{\prime} \in h^{\prime}$, controlled by agents $i j$ and $i k$ respectively. Let $a$ be the unique action on this path leaving $x$. Since $|A(h)| \geq 2$, we can find another action, say $b$ at $x$. Next, choose two different actions $a^{\prime}$ and $b^{\prime}$ at $x^{\prime}$.

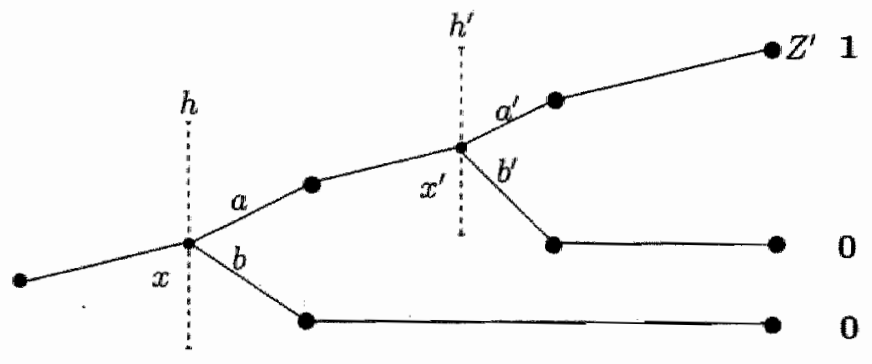

Figure 7.5

Note that the line from the root to $x$ does not represent one single action. This line covers all the actions and chance moves that are present on the path to $x$. The same holds for the line ending at $x^{\prime}$ and the lines ending at terminal nodes.

By $Z^{\prime}$ we denote the set of terminal nodes which follow the action $a^{\prime}$. Let the payoff function $u$ be given by

$$
u_{j}(z):= \begin{cases}1 & \text { if } j=i \text { and } z \in Z^{\prime} \\ 0 & \text { otherwise }\end{cases}
$$

and let $\Gamma$ be the garne $\langle\mathcal{S}, u\rangle$. Choose a pure strategy profile $m$ in $\Gamma^{\pi}$ such that

(1) $m$ chooses all the actions on the path from the root to $x^{\prime}$, except $a$ and

(2) $m$ chooses the actions $b$ and $b^{\prime}$.

Then $m$ is a Nash equilibrium for the game $\Gamma^{\pi}$ which gives player i payoff 0 . 
Let $f$ be an arbitrary transformation function. It can be checked easily that $f^{-1}(m)=\{m\}$. However, $m$ is not a Nash equilibrium in $\Gamma$ since player $i$ can strictly increase his payoff by deviating unilaterally to a pure strategy selecting the actions $a$ and $a^{\prime}$. Consequently, the Nash equilibrium correspondence is not invariant under $\pi$.

The other solution concepts

We show that perfect equilibria, proper equilibria, KM-stable sets and Mstable sets are not weakly invariant under any dependent player splitting. In order to prove these results, we use one and the same counterexample.

Lemma 7.6.2 Perfect equilibria, proper equilibria, KM-stable sets and $M$ stable sets are not weakly invariant under any dependent player splitting.

Proof. Let $\mathcal{S}$ be an extensive form structure and $\pi$ a dependent player splitting on $\mathcal{S}$. Then we can choose $h, h^{\prime}, x, x^{\prime}, a, b, a^{\prime}, b^{\prime}$ as in Lemma 7.6.1.

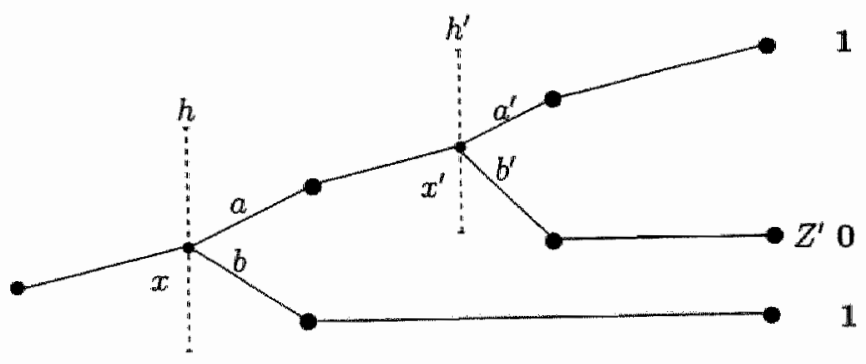

Figure 7.6

By $Z^{\prime}$ we denote the set of those terminal nodes following the action $b^{\prime}$. Let the payoff function $u$ be given by

$$
u_{j}(z):= \begin{cases}0 & \text { if } j=i \text { and } z \in Z^{i} \\ 1 & \text { otherwise }\end{cases}
$$


and let $\Gamma=\langle\mathcal{S}, u\rangle$. Choose a pure strategy profile $m$ such that (1) all the actions on the path to $x^{\prime \prime}$, except $a$, are chosen and

(2) the actions $b$ and $b^{\prime}$ are chosen.

We show that $\{m\}$ is a M-stable set in $\Gamma$. Since a M-stable set always contains a proper equilibrium, it follows that $m$ is proper (and therefore perfect) in $\Gamma$. Moreover, we may conclude that $\{m\}$ is a KM-stable set in $\Gamma$.

Secondly, we prove that for every transformation function $f, f(m)$ is not a perfect equilibrium in $\Gamma^{\pi}$. Consequently, $f(m)$ is not proper in $\Gamma^{\pi}$ and the set $f(\{m\})$ is not a KM-stable set and not a M-stable set in $\Gamma^{\pi}$ since KMand $\mathrm{M}$-stable sets consist solely of perfect equilibria.

Combining all the insights above leads to the conclusion that perfect equilibria, proper equilibria, KM-stable sets and M-stable sets are not weakly invariant under $\pi$.

In order to show that $\{m\}$ is a M-stable set in $\Gamma$, consider the map $\rho$ which assigns to every mistake vector $\eta$ the MSP $\rho(\eta)$ given by

$$
\rho(\eta)_{j}\left(r_{j}\right)= \begin{cases}\eta_{j}\left(r_{j}\right) & \text { if } r_{j} \neq m_{j} \\ 1-\sum_{s_{j} \neq m_{j}} \eta_{j}\left(s_{j}\right) & \text { if } r_{j}=m_{j}\end{cases}
$$

for each player $j$.

It can be checked easily that $\rho(\eta) \in N E\left(\Gamma^{\eta}\right)$ for all $\eta$. Since $\rho$ is affine, it follows that graph $(\rho)$ is a semi-algebraic set. Next, we show that the projection $\tau$ from graph $(p)$ to the space of mistake vectors is not relatively null-homotopic. We restrict ourselves to a short outline of the proof. To this purpose, consider the map $\bar{\rho}$ which assigns to every mistake vector $\eta$ the pair $(\eta, \rho(\eta))$. Clearly, $\bar{\rho}$ is continuous and is equal to the inverse of $\tau$. Therefore, $\tau$ is not relatively null-homotopic (see, for instance, Munkres, 1984). Furthermore, the dimension of $\operatorname{graph}(\rho)$ is equal to the dimension of the space of mistake vectors. Therefore, we may conclude that $\{m\}$ is a M-stable set.

Now, let $f$ be a corresponding transformation function. Then, $f(m)=m$. Let $i k$ be the agent controlling information set $h^{\prime}$. The pure strategy $m_{i k}$ played by agent $i k$ in $m$ is weakly dominated by the pure strategy $m_{i k} \backslash a^{\prime}$ in $\Gamma^{\pi}$. Therefore, $m$ is not a perfect equilibrium in $\Gamma^{\pi}$.

\subsection{Characterization of independent player split- tings and single appearance structures}

By combining the results of section 4 and 6 , we obtain the following characterizations of the class of independent player splittings. 
Theorem 7.7.1 The class of independent player splittings is the largest class of player splittings under which

(a) the Nash equilibria, perfect equilibria, KM-stable sets and M-stable sets are invariant and

(b) the proper equilibria are weakly invariant.

By largest, we mean largest with respect to set inclusion.

Next, we consider a special class of extensive form structures which we call single appearance structures. An extensive form structure is called a single appearance structure if every path in the game tree crosses at most one information set of every player. In other words, each player appears at most one single time in every play of the game. Obviously, the class of single appearance structures can be characterized by stating that it is the largest class of structures for which every player splitting is independent.

Using the theorem above, we can give the following characterizations of the class of single appearance structures in terms of invariance of solution concepts under player splittings.

Theorem 7.7.2 The class of single appearance structures is the largest class of extensive form structures $\mathcal{S}$ for which

(a) the Nash equilibria, perfect equilibria, KM-stable sets and M-stable sets are invariant under every player splitting on $\mathcal{S}$ and

(b) the proper equilibria are weakly invariant under every player splitting on $\mathcal{S}$. 



\section{Chapter 8}

\section{Limit consistent solutions in non-cooperative games}

\subsection{Introduction}

This chapter is based on Perea y Monsuwé and Peters (1996b).

The concept of consistency has been widely applied in game theory. Globally speaking, game-theoretic solution is called consistent if it is invariant in the reduced game for a subset of the players given that the other players are kept: at the solution outcome in the original game. Here, an outcome may be a payof vector (usually in a cooperative game) or a strategy vector (usually in a noncooperative game). Obviously, this global definition leaves much freedom for formalization, by varying the definition of the reduced game. For many different solutions, reduced games have been proposed with respect to which the solution is consistent.

The paper by Peleg and Tijs (1996) was the first to apply the consistency concept to noncooperative games. For a strategy profile in a normal form game and a coalition, a natural reduced game arises by fixing the players outside the coalition at their strategies as given in the profile. Obviously, a Nash equilibrium will induce a Nash equilibrium in the reduced game. Conversely, if in every reduced game the induced strategy profile is a Nash equilibrium, then the original profile must be a Nash equilibrium. We express this by saying that the Nash equilibrium correspondence is consistent as well as conversely consistent. For a survey on reduced noncooperative games, consistency and converse consistency, see Peleg and Tijs (1996). In this chapter, the combination of consistency and converse consistency is termed strong consistency. Once a solution is strongly consistent, it is completely determined by its behavior on one-person games. Hence, the Nash equilibrium 
correspondence for normal form games is the unique strongly consistent solution assigning the set of expected payoff maximizing strategies to one-player games. This is the main result in Peleg and Tijs (1996). By varying the behavior of a solution on one-person games, other strongly consistent solutions are obtained. Patrone et al. (1995) call these solutions personalized Nash equilibria. Basically, by defining one-person behavior and imposing strong consistency, a best response correspondence is defined and extended to an equilibrium concept.

In this chapter, we aim to contribute to the study of consistency in finite noncooperative games in the following ways.

First, we provide sufficient conditions on one-person behavior such that a corresponding strongly consistent solution is nonempty. Observe that the pure Nash equilibrium correspondence cannot satisfy these conditions, because it is strongly consistent but may be empty. In fact, Norde et al. (1996) have proved that there exists no proper refinement of the Nash equilibrium correspondence that is consistent and nonempty-let alone strongly consistent. This result applies in particular to familiar refinements like perfect and proper Nash equilibrium, which both are nonempty and therefore cannot be consistent. As an approach to this problem we introduce, second, the concept of limit consistency. A solution is limit consistent, roughly, if it can be approximated by strongly consistent solutions. A limit consistent solution is generated by a class of one-person solutions rather than by a unique one-person solution. It will be shown that both the perfect and the proper equilibrium correspondences are limit consistent. Third, we extend the concept of limit consistency to solutions for games in extensive form. We show that the correspondences of weakly perfect equilibrium and of sequential equilibrium (Kreps and Wilson, 1982) are limit consistent.

The organization of the chapter is as follows. In Section 2 we introduce strong consistency for solutions in normal form games, and in Section 3, among other things, we provide conditions for nonemptiness of strongly consistent solutions. In Section 4 we introduce limit consistency, and provide a necessary and sufficient condition for nonemptiness of limit consistent solutions. The perfect and proper equilibrium correspondences are proved to be limit consistent in Section 5. In Section 6 extensive form games and the corresponding extensions of strong and limit consistency are defined. Sections 7 and 8 give the mentioned results on weakly perfect and sequential equilibria, respectively. 


\title{
8.2 Strongly consistent solutions in normal form games
}

\author{
Normal form games and reduced games
}

Let $\Gamma=\langle N, M, v\rangle$ be a normal form game with player set $N$, where $M=$ $x_{i \in N} M_{i}$ denotes the space of pure strategy profiles and $v=\left(v_{i}\right)_{i \in N}$ is a collection of payoff functions $v_{i}: M \rightarrow \mathbb{R}$. We assume that the pure stratiegy spaces $M_{i}$ are all finite. In the remainder of this chapter, we write $\langle M, v\rangle$ instead of $\langle N, M, v\rangle$ if there can be no misunderstanding about the player set. Moreover, we write "game" instead of "normal form game" if it is clear that we are talking about normal form games.

A mixed strategy for player $i$ is a probability distribution $p_{i}$ on the set $M_{i}$ of pure player $i$ strategies. A combination $p=\left(p_{i}\right)_{i \in N}$ of mixed strategies $p_{i}$ is called a mixed strategy profile (MSP). The expected payoff for player $i$ if the MSP $p$ is played is denoted by $v_{i}(p)$.

An MSP $p$ is called completely mixed if $p_{i} \in \Delta^{0}\left(M_{i}\right)$ for every $i$. Here, $\Delta^{0}\left(M_{i}\right)$ denotes the set of probability distributions on $M_{i}$ which put strictly positive weight on every $m_{i} \in M_{i}$.

By $B_{i}(p, \Gamma)$, we denote the set of player i's pure best responses against the MSP $p$, i.e.

$$
B_{i}(p, \Gamma):=\left\{m_{i} \mid v_{i}\left(p \backslash m_{i}\right) \geq v_{i}\left(p \backslash l_{i}\right) \text { for every } l_{i} \in M_{i}\right\}
$$

Here, $p \backslash m_{i}$ is the MSP which we obtain if the mixed strategy $p_{i}$ is replaced by the pure strategy $m_{i}$ in $p$.

Let $\Gamma=\langle M, v\rangle$ be a game, $p$ an MSP and $T$ a coalition of players. By $\Gamma^{T}$, , we denote the reduced game $\left\langle T, M^{\prime}, v^{\prime}\right\rangle$ where $M^{\prime}=x_{i \in T} M_{i}$ and $v_{i}^{\prime}\left(p^{\prime}\right)=$ $v_{i}\left(p^{\prime}, p_{N \backslash T}\right)$ for all $i \in T$ and all MSP's $p^{\prime}=\left(p_{i}^{i}\right)_{i \in T}$.

Here, $p_{N \backslash T}$ denotes the restriction of the MSP $p$ on the players in $N \backslash T$. Hence, $\Gamma^{T, p}$ is the game with player set $T$ which we obtain if we assume that: players outside $T$ play according to $p$.

A family $\mathcal{G}$ of normal form games is called closed if for every $\Gamma$ in $\mathcal{G}$, every nonempty coalition $T \subset N$ and every MSP $p$, the reduced game $\Gamma^{T, p}$ is also in $\mathcal{G}$. 


\section{Strongly consistent solutions}

A solution on a family of normal form games is a correspondence which assigns to every game in this family a set of MSP's in this game. For a game $\Gamma$ in this family, we call $\varphi(\Gamma)$ the set of solution profiles in this game.

Let $G$ be a closed farnily of normal form games and $\varphi$ a solution on $\mathcal{G}$. For a game $\Gamma$ in $\mathcal{G}$ containing at least two players we define

$$
\tilde{\varphi}(\Gamma):=\left\{p \mid p_{T} \in \varphi\left(\Gamma^{T}\right) \text { for every } \emptyset \subset T_{\neq} T_{\neq}\right\}
$$

In other words, $\tilde{\varphi}(\Gamma)$ is the set of MSP's such that for every nonempty coalition, the "reduced" profile is a solution profile of the reduced game.

For one-player games $\Gamma$, we define $\tilde{\varphi}(\Gamma):=\varphi(\Gamma)$. The reason for this definition is the fact that there are no coalitions $\emptyset \underset{\neq}{\subset} \underset{f}{\subseteq}$ in one-player games.

A solution $\varphi$ is called consistent (CONS) if $\varphi(\Gamma) \subset \tilde{\varphi}(\Gamma)$ for every $\Gamma$ in $\mathcal{G}$. This means that for every solution profile in $\Gamma$ and every non-trivial subset of players, the reduced solution profile is a solution of the reduced game.

A solution $\varphi$ is called conversely consistent (COCONS) if $\varphi(\Gamma) \subset \varphi(\Gamma)$ for every $\Gamma$ in $\mathcal{G}$ with at least two players.

This means that an MSP which has the property that for every non-trivial subset of players the reduced profile is a solution profile of the reduced game, should be a solution profile itself.

Note that the properties CONS and COCONS together are equivalent to the property $\varphi(\Gamma)=\bar{\varphi}(\Gamma)$ for every $\Gamma$ in $\mathcal{G}$.

We call a solution strongly consistent if it satisfies CONS and COCONS.

\subsection{Properties of strongly consistent solutions}

In Patrone et al. (1995), it is shown that every strongly consistent solution is completely determined by its behavior on one-person games. In fact, the behavior of a strongly consistent solution on the class of one-person games induces an optimal response correspondence, which assigns to every MSP $p$ a set of MSP's which are optimal responses against $p$. Here, we use the expression optimal response instead of best response since the latter term is reserved to denote payoff-maximizing responses. Hence, best responses are in our notation a special case of optimal responses. In the following lemma, it is shown that the class of strongly consistent solutions consists exactly of 
those solutions which assign to a game the set of MSP's which are optimal responses against themselves.

Lemma 8.3.1 A solution $\varphi$ is strongly consistent if and only if for every game $\Gamma$,

$$
\varphi(\Gamma)=\left\{p \mid p_{i} \in \varphi\left(\Gamma^{\{i\}, p)} \text { for every } i\right\} .\right.
$$

There is a close relationship between this lemma and a result in Patrone et al. (1995), which states that a solution is a personalized Nash equilibrium correspondence if and only if it satisfies personalized one-person rationality, CONS and COCONS. This relationship stems from the fact that the class of personalized Nash equilibrium correspondences contains exactly those solutions assigning to every game $\Gamma$ the set of MSP's

$$
\left\{p \mid p_{i} \in \varphi_{i}^{1}\left(\Gamma^{\{i\}, p}\right) \text { for every } i\right\}
$$

where $\varphi_{i}^{1}$ is an arbitrary but fixed solution on one-person games for every $i$. Proof of Lemma 8.3.1. (a) First, we show the implication from left to right.

Let $\varphi$ be a strongly consistent solution. We define the solution $\psi$ by

$$
\psi(\Gamma)=\left\{p \mid p_{i} \in \varphi\left(\Gamma^{\{i\}, w}\right) \text { for every } i\right\}
$$

for every game $\Gamma$ and show that $\varphi=\psi$.

Because $\varphi$ is consistent, it follows that $\varphi(\Gamma) \subset \psi(\Gamma)$ for every game $\Gamma$.

The other inclusion will be shown by induction on the number of players in $\Gamma$.

If $\Gamma$ is a one-player game, the inclusion $\psi(\Gamma) \subset \varphi(\Gamma)$ holds by definition. Now, assume that $\psi\left(\Gamma^{\prime}\right) \subset \varphi\left(\Gamma^{\prime}\right)$ for every game $\Gamma$ with strictly less than $n$ players and consider a game $\Gamma$ with $n$ players.

Choose an arbitrary profile $p \in \psi(\Gamma)$ and let $\emptyset \subsetneq_{F}{ }$. By definition, we have $p_{T} \in \psi\left(\Gamma^{T, p}\right)$. Using the induction assumption, it follows that $p_{T} \in$ $\varphi\left(\Gamma^{T, p}\right)$. Since this holds for every such $T$, the converse consistency of $\varphi$ implies that $p \in \varphi(\Gamma)$.

(b) The implication from right to left is obvious.

Intuitively, the lemma says that a solution is strongly consistent if and only if it assigns to every game the set of strategy profiles in which every strategy is an optimal response. Therefore, the behavior of a strongly consistent solution is similar to that of the Nash equilibrium correspondence. This is a reason 
why strongly consistent solutions are called personalized Nash equilibria in Patrone et al. (1995).

The observations above enable us to construct a mechanism which produces strongly consistent solutions. The mechanism works in the following way.

First, choose a one-person solution $\varphi^{1}$ which assigns to every one-person game a set of mixed strategies.

For every game $\Gamma$, the optimal response correspondence induced by the oneperson solution $\varphi^{1}$ is the function $f$ assigning to every player $i$ and every MSP $p$ the set $\varphi^{1}\left(\Gamma^{(i), p}\right)$.

Finally, define the solution $\varphi$ to be the solution which assigns to every normal form game the set of MSP's in which every mixed strategy is an optimal response (w.r.t. $\varphi^{\mathbb{1}}$ ) against the strategies of the other players. Formally, we obtain the following definition.

The solution $\varphi$ given by

$$
\varphi(\Gamma):=\left\{p \mid p_{i} \in \varphi^{1}\left(\Gamma^{\{i\}_{i p}}\right) \text { for every } i\right\}
$$

is called the strongly consistent solution generated by the one-person solution $\varphi^{1}$.

The Nash equilibrium correspondence, for example, is a strongly consistent solution generated by the one-person solution which assigns to every oneperson game the set of payoff maximizers. A proof for this result can be found in Peleg and Tijs (1996).

In general, however, the solution $\varphi$ generated by the mechanism described above may be empty in some games, even when the one-person solution $\varphi^{1}$ is always nonempty. To illustrate this fact, consider the one-person solution $\varphi^{1}$ which assigns to every one-person game the set of pure strategies with maximal payoff. Obviously, the solution generated by $\varphi^{\mathbb{1}}$ assigns to every game the set of pure Nash equilibria. However, it is well-known that pure Nash equilibria do not always exist.

In the following theorem, we describe a sufficient condition to generate a nonempty, strongly consistent solution. By nonempty, we mean that the solution assigns to every game a nonempty set of MSP's. In order to state the theorem, we need the definition of upper-hemicontinuity of a one-person solution.

A one-person solution $\varphi^{1}$ is called upper-hemicontinuous if for every oneperson game $\Gamma=\left\langle M_{i}, v_{i}\right\rangle$, every sequence $\Gamma^{k}=\left\langle M_{i}, v_{i}^{k}\right\rangle$ of games converging to $\Gamma$ and every sequence $p^{k}$ of MSP's converging to $p$ with $p^{k} \in \varphi^{\mathbb{I}}\left(\Gamma^{k}\right)$ we have $p \in \varphi^{\mathbb{1}}(\Gamma)$. 
In the proof of the theorem, we also need another kind of upper-hemicontinuity, namely the upper-hemicontinuity of optimal response correspondences.

The optimal response correspondence induced by $\varphi^{1}$ is upper-hemicontinutous if for every sequence $p^{k}$ of MSP"s converging to $p$ and every sequence $q_{i}^{k} \in$ $\varphi^{1}\left(\Gamma^{\{i\}} p^{p^{k}}\right)$ converging to $q_{i}$ for all $i$ it holds that $q_{i} \in \varphi^{\frac{1}{1}}\left(\Gamma^{\{i\}^{3}}\right)$ for all $i$.

In words, if a sequence of strategies consists of optimal responses against a sequence of MSP's, then the limit strategy is an optimal response against the limit MSP.

Observe that both types of upper-hemicontinuity are based on the usual closed-graph definition of upper-hemicontinuity of a correspondence. A oneperson solution, however, is regarded as a correspondence on a class of games, whereas the optimal response correspondence varies with the strategy profiles within the same game.

Theorem 8.3.2 Let $\varphi$ be a strongly consistent solution generated by the one-person solution $\varphi^{1}$. If $\varphi^{1}$ is upper-hemicontinuous and assigns to every one-person game a nonempty, convex and compact set of strategies, then $\varphi$ is nonempty.

Proof. Let $\Gamma=\langle M, v\rangle$ be a normal form game. First, we show that the optimal response correspondence in $\Gamma$ induced by $\varphi^{1}$ is upper-hemicontinuous. Let $p^{k}$ be a sequence of MSP's converging to $p$ and $q_{i}^{k} \in \varphi^{\mathrm{l}}\left(\Gamma^{\{i\}} p^{p^{k}}\right)$ a sequence of optimal responses converging to $q_{i}$. For every $k$, define the game $\Gamma^{i, k}$ by $\Gamma^{i, k}:=\Gamma^{\{i\}, p^{k}}$. Obviously, the sequence $\Gamma^{i, k}$ converges to $\Gamma^{\{i\}}, p$. By the upper-hemicontinuity of $\varphi^{1}$ we know that $q_{i} \in \varphi^{1}\left(\Gamma^{\{i}\right\}$ ) which implies that the optimal response correspondence is upper-hemicontinuous.

Since, by assumption, the optimal response correspondence assigns to every MSP $p$ a nonempty, convex and compact set of MSP's, we can apply Kakutani's theorem to ensure the existence of a fixed point of the optimal response correspondence in the game $\Gamma$. Since the solutions in $\varphi(\Gamma)$ are exactly those fixed points, the nonemptiness of $\varphi$ is estatilished.

Example 8.1. Let $\varphi^{1}$ be the one-person solution assigning to every oneperson game $\Gamma=\left\langle M_{i}, v_{i}\right\rangle$ the set of MSP's $p$ with $v_{i}(p)$ minimal. Since it can be checked easily that $\varphi^{1}$ satisfies all the conditions in the theorem above, it follows that the strongly consistent solution generated by $\varphi^{\mathbb{1}}$ is nonempty.

A solution which assigns to every game a subset of the set of Nash equilibria is called a Nash equilibrium refinement. The following result, which has been proved in Norde et al. (1996), shows that it is impossible to find a nonempty, consistent Nash equilibrium refinement other than the Nash equilibrium correspondence itself. 
Theorem 8.3.3 Let $\varphi$ be a nonempty Nash equilibrium refinement satisfying CONS. Then, $\varphi$ is equal to the Nash equilibrium correspondence.

This theorem implies, in particular, that there does not exist a nonempty, strongly consistent solution which is a strict refinement of the Nash equilibrium correspondence. This is an important reason to introduce a new mechanism in the next section, which enables us to generate so-called limit consistent solutions. In particular, Nash equilibrium refinements such as perfect equilibria, proper equilibria, weakly perfect and sequential equilibria can be generated by this mechanism.

\subsection{Limit consistent solutions}

In this section we concentrate on solutions which need not be strongly consistent, but have the property that every solution point can be approximated by a sequence of solution points of strongly consistent solutions. We call such solutions limit consistent.

For a formal definition of limit consistent solutions, we need the notion of a so-called one person solution function.

A one-person solution function is a function $\Phi^{1}$ which assigns to every $\varepsilon>0$ a one-person solution $\varphi^{1}=\Phi^{1}(\varepsilon)$.

Intuitively, the number $\varepsilon$ can be interpreted as a perturbation factor and the one-person solution $\Phi^{1}(\varepsilon)$ can be seen as a perturbed one-person solution.

For every $\varepsilon>0$ the unique strongly consistent solution generated by the oneperson solution $\Phi^{1}(\varepsilon)$ is denoted by $\Phi(\varepsilon)$. The function $\Phi$ is called a solution function.

A solution $\varphi$ is called limit consistent if there is a one-person solution function $\Phi^{\mathbb{1}}$ such that

$\varphi(\Gamma)=\left\{p \mid \begin{array}{l}\text { there is a sequence } \varepsilon^{k}>0 \text { converging to } 0 \text { and a sequence } \\ p^{k} \in \Phi\left(\varepsilon^{k}\right)(\Gamma) \text { converging to } p\end{array}\right\}$.

In this case, we say that the limit consistent solution is generated by the one-person solution function $\Phi^{1}$.

Every one-person solution function $\Phi^{1}$ induces a solution function $\bar{\Phi}$ given by

$$
\bar{\Phi}(\varepsilon)(\Gamma):=\bigcup_{\delta \in(0, \varepsilon]} \Phi(\delta)(\Gamma)
$$

for every game $\Gamma$ and every $\varepsilon>0$. 
Theorem 8.4.1 The following two statements are equivalent:

(1) $\varphi$ is a limit consistent solution generated by the one-person solution function $\Phi^{1}$.

(2) $\varphi(\Gamma)=\bigcap_{\varepsilon>0} c l(\tilde{\Phi}(\varepsilon)(\Gamma))$ for every game $\Gamma$.

Here, $\operatorname{cl}(\bar{\Phi}(\varepsilon)(\Gamma))$ denotes the closure of the set $\bar{\Phi}(\varepsilon)(\Gamma)$.

Proof. We only show the implication from (1) to (2), the other implication can be shown in a similar way.

Let $\varphi$ be a limit consistent solution generated by $\Phi^{1}$ and let $\Gamma$ be a game. We prove the coincidence of both sets in (2) by the double inclusion argument.

(a) Show that $\varphi(\Gamma) \subset \bigcap_{\varepsilon>0} \operatorname{cl}(\bar{\Phi}(\varepsilon)(\Gamma))$.

Let $p \in \varphi(\Gamma)$ and let $\varepsilon>0$ be given. By definition of limit consistency, there is a sequence $\varepsilon^{k}>0$ converging to 0 and a sequence $p^{k} \in \Phi\left(\varepsilon^{k}\right)(\Gamma)$ converging to $p$. Obviously, $p^{k} \in \Phi(\varepsilon)(\Gamma)$ for $k$ large enough, which implies that $p \in \mathrm{cl}(\bar{\Phi}(\varepsilon)(\Gamma))$. Since this holds for every $\varepsilon>0$, it follows that $p \in \bigcap_{\varepsilon>0}$ $\operatorname{cl}(\bar{\Phi}(\varepsilon)(\Gamma))$.

(b) Show that $\bigcap_{\varepsilon>0} \operatorname{cl}(\bar{\Phi}(\varepsilon)(\Gamma)) \subset \varphi(\Gamma)$.

Let $p \in \bigcap_{\varepsilon>0} \operatorname{cl}(\bar{\Phi}(\varepsilon)(\Gamma))$. Then $p \in \operatorname{cl}\left(\bar{\Phi}\left(\frac{1}{k}\right)(\Gamma)\right)$ for every $k$, so for every $k$ there is a sequence $\left(p^{k, l}\right)_{l \in \mathbb{N}} \in \bar{\Phi}\left(\frac{1}{k}\right)(\Gamma)$ with $p=\lim _{l \rightarrow \infty} p^{k, l}$. By definition, there is a number $\varepsilon(k, l) \in\left[0, \frac{1}{k}\right]$ for every $k, l$ with $p^{k, l} \in \Phi(\varepsilon(k, l))(\Gamma)$. For every $k$, choose an integer $l(k)$ with $\left\|p-p^{k, l(k)}\right\| \leq \frac{1}{k}$. By construction, we obtain $p^{k, l(k)} \in \Phi(\varepsilon(k, l(k)))(\Gamma)$ and $p=\lim _{k \rightarrow \infty} p^{k, l(k)}$. Because $p^{k, l(k)}$ converges to $p$ and $\varepsilon^{k, l(k)}$ converges to 0 as $k \rightarrow \infty$, we conclude that $p \in \varphi(\Gamma)$.

We say that a solution is closed if it assigns to every game a closed set of MSP's.

From Theorem 8.4.1 it follows that a limit consistent solution assigns to every game $\Gamma$ the set $\bigcap_{\varepsilon>0} \operatorname{cl}(\tilde{\Phi}(\varepsilon)(\Gamma))$, which is obviously closed. Hence, we obtain the following corollary.

Corollary 8.4.2 Every limit consistent solution is closed.

It turns out that nonemptiness of limit consistent solutions can be characterized by nonemptiness of the corresponding solution functions $\bar{\Phi}$.

Theorem 8.4.3 Let $\varphi$ be a limit consistent solution generated by the oneperson solution function $\Phi^{1}$. Then, $\varphi$ is nonempty if and only if $\bar{\Phi}(\varepsilon)$ is nonempty for every $\varepsilon>0$. 
Proof. First, we prove the implication from left to right.

Let $\varphi$ be a nonempty limit consistent solution generated by $\Phi^{1}$ and $\Gamma$ an arbitrary game. By Theorem 8.4.1, we know that $\varphi(\Gamma)=\bigcap_{\varepsilon>0} \mathrm{cl}(\bar{\Phi}(\varepsilon)(\Gamma))$, which implies that $\mathrm{cl}(\bar{\Phi}(\varepsilon)(\Gamma)) \neq \emptyset$ for every $\varepsilon>0$. This is only possible when $\Phi(\varepsilon)(\Gamma) \neq \emptyset$ for every $\varepsilon>0$.

Now, consider the direction from right to left.

Let the solution $\Phi(\varepsilon)$ be nonempty for every $\varepsilon>0$ and let $\Gamma$ be a game. Then, the set $\mathrm{cl}(\bar{\Phi}(\varepsilon)(\Gamma))$ is compact for every $\varepsilon>0$. Since, by construction, $\mathrm{cl}(\bar{\Phi}(\varepsilon)(\Gamma)) \subset \mathrm{cl}\left(\bar{\Phi}\left(\varepsilon^{\prime}\right)(\Gamma)\right)$ whenever $\varepsilon \leq \varepsilon^{\prime}$ it follows by Theorem 8.4.1 that $\varphi(\Gamma)=\bigcap_{\varepsilon>0} \operatorname{cl}(\bar{\Phi}(\varepsilon)(\Gamma)) \neq \emptyset$.

This theorem and the definition of $\bar{\Phi}(\varepsilon)(\Gamma)$, imply a characterization of the class of nonempty limit consistent solutions in terms of the solutions $\Phi(\varepsilon)$.

Corollary 8.4.4 Let $\varphi$ be a limit consistent solution generated by the oneperson solution function $\Phi^{1}$. Then, $\varphi$ is nonempty if and only if for every $\varepsilon>0$ there is a $\delta \in(0, \varepsilon]$ with $\Phi(\delta)$ nonempty.

Example 8.2. Consider the one-person solution function $\Phi^{1}$ which assigns to every $\varepsilon>0$ and every one-person game $\Gamma=\left\langle M_{i}, v_{i}\right\rangle$ the set

$$
\Phi^{1}(\varepsilon)(\Gamma):= \begin{cases}\left\{p \mid v_{i}(p) \text { minimal }\right\} & \text { if } \varepsilon \in \mathbb{Q} \\ \emptyset & \text { otherwise }\end{cases}
$$

The strongly consistent solution $\Phi(\varepsilon)$ generated by $\Phi^{l}(\varepsilon)$ is nonempty if $\varepsilon \in \mathbb{Q}$. Hence, in view of the corollary above, the limit consistent solution $\varphi$ generated by $\Phi^{1}$ is nonempty despite the fact that $\Phi(\varepsilon)$ is empty for all $\varepsilon \notin \mathbb{Q}$

\subsection{Perfect and proper equilibria}

One of the possible ways to define perfect equilibria is by saying that a perfect: equilibrium can be approximated by a sequence of so called $\varepsilon$-perfect strategy profiles.

(a) Let $\varepsilon>0$ and $\Gamma$ a normal form game. An MSP $p$ is called $\varepsilon$-perfect if $p$ is completely mixed and $v_{i}\left(p \backslash m_{i}\right)<v_{i}\left(p \backslash m_{i}^{\prime}\right)$ implies $p_{i}\left(m_{i}\right) \leq \varepsilon$.

(b) An MSP $p$ is a perfect equilibrium if there is a sequence $\varepsilon^{k}$ of positive numbers converging to 0 and a sequence $p^{k}$ of $\varepsilon^{k}$-perfect profiles converging
to $p$. 
Theorem 8.5.1 The perfect equilibrium correspondence is a limit consistent solution generated by the one-person solution function $\Phi^{1}$ given by

$$
\Phi^{1}(\varepsilon)(\Gamma)=\left\{p_{i} \in \Delta^{0}\left(M_{i}\right) \mid v_{i}\left(m_{i}\right)<v_{i}\left(m_{i}^{\prime}\right) \text { implies } p_{i}\left(m_{i}\right) \leq \varepsilon\right\}
$$

for every $\varepsilon>0$ and every one-person game $\Gamma=\left\langle M_{i}, v_{i}\right\rangle$.

The proof of this result is straightforward.

(a) An MSP $p$ is called an $\varepsilon$-proper profile if $p$ is completely mixed and $v_{i}\left(p \backslash m_{i}\right)<v_{i}\left(p \backslash m_{i}^{\prime}\right)$ implies $p_{i}\left(m_{i}\right) \leq \varepsilon p_{i}\left(m_{i}^{\prime}\right)$.

(b) An MSP $p$ is called a proper equilibrium if there is a sequence $\varepsilon^{k}$ converging to 0 and a sequence $p^{k}$ of $\varepsilon^{k}$-proper profiles converging to $p$.

Similarly to perfect equilibria, we can show that the proper equilibrium correspondence is limit consistent.

Theorem 8.5.2 The proper equilibrium correspondence is a limit consistent solution generated by the one-person solution function $\Phi^{1}$ given by

$$
\Phi^{1}(\varepsilon)(\Gamma)=\left\{p_{i} \in \Delta^{0}\left(M_{i}\right) \mid v_{i}\left(m_{i}\right)<v_{i}\left(m_{i}^{\prime}\right) \text { implies } p_{i}\left(m_{i}\right) \leq \varepsilon p_{i}\left(m_{i}^{\prime}\right)\right\}
$$

for every $\varepsilon>0$ and every one-person game $\Gamma=\left\langle M_{i}, v_{i}\right\rangle$.

\subsection{Strongly and limit consistent solutions in extensive form games}

Reduced extensive form games, strongly and limit consistent solutions

Let $\Gamma$ be an extensive form game, $T \subset N$ a subset of players and $\sigma$ a BSP. Then, the reduced game $\Gamma^{T, \sigma}$ is the extensive form game which we obtain by splitting every information set $h$ controlled by a player outside $T$ into a collection of chance nodes at which the moves of nature are given by the probability distribution $\sigma_{h}$. If an action is played with probability zero in $\sigma_{h}$, then the corresponding edges are deleted in the game tree. In this way we assure that the probability distributions of the moves of nature are strictly positive. The remaining players in the reduced game are the players in $T$.

A solution for extensive form garnes is a correspondence which assigns to every extensive form game a set of BSP's.

An assessment solution for extensive form games is a correspondence which assigns to every extensive form game a set of assessments . 
Let $\varphi$ be a solution. For a game $\Gamma$ in $\mathcal{G}$ we define

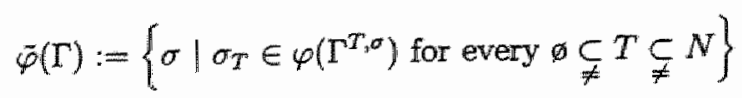

where $\sigma_{T}$ is the restriction of $\sigma$ on the information sets controlled by players in $T$.

If $\varphi$ is an assessment solution, we define

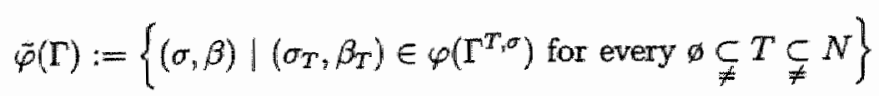

where $\left(\sigma_{T}, \beta_{T}\right)$ is the restriction of $(\sigma, \beta)$ on the information sets controlled by players in $T$.

In the obvious way, we define consistency (CONS) and converse consistency (COCONS) for solutions and assessment solutions. Again, we call a solution (or assessment solution) strongly consistent, if it is both consistent and conversely consistent.

The definition of limit consistent solutions (assessment solutions) for extensive form games is given in the obvious way.

\subsection{Weakly perfect equilibria}

A BSP $\sigma$ is called a weakly perfect equilibrium in an extensive form game $\Gamma=\langle\mathcal{S}, u\rangle$ if there is a sequence of games $\Gamma^{k}=\left\langle\mathcal{S}, u^{k}\right\rangle$ converging to $\Gamma$ and a sequence of completely mixed BSP's $\sigma^{k}$ converging to $\sigma$ such that $a \notin B_{h}\left(\sigma^{k}, \Gamma^{k}\right)$ for some $k$ implies $\sigma_{h}(a)=0$ for all $a \in A(h)$ and $h \in H_{i}$. Here, $B_{h}\left(\sigma^{k}, \Gamma^{k}\right)$ denotes the set of pure best responses (actions) of agent $h$ against $\sigma^{k}$ in $\Gamma^{k}$.

This definition of weakly perfect equilibria can be found in Kreps and Wilson (1982). It can be seen easily that every perfect equilibrium of the agent normal form of $\Gamma$ is weakly perfect by choosing $\Gamma^{k}$ equal to $\Gamma$.

In the following theorem, we show that the weakly perfect equilibrium concept is limit consistent. In order to prove this result, we need two more definitions.

For two extensive form games $\Gamma=\langle\mathcal{S}, u\rangle$ and $\Gamma^{\prime}=\left\langle\mathcal{S}, u^{\prime}\right\rangle$ with the same extensive form structure, we define the distance $d\left(\Gamma, \Gamma^{i}\right)$ by

$$
d\left(\Gamma, \Gamma^{\prime}\right):=\max _{i, z}\left|u_{i}(z)-u_{i}^{i}(z)\right| .
$$


In an extensive form game $\Gamma$, the BSP $\sigma$ is called an $\varepsilon$-perfect equilibrium if $\sigma$ is completely mixed and $\left.U_{i}(\sigma \backslash a\rangle\right) U_{i}(\sigma \backslash b)$ implies $\sigma_{h}(a) \leq \varepsilon$ for every $h \in H_{i}$ and all $a, b \in A(h)$.

Here, $U_{i}$ denotes the expected payoff.

Theorem 8.7.1 The weakly perfect equilibrium correspondence is a limit consistent solution generated by the one-person solution function $\Phi^{11}$ given by

$$
\Phi^{1}(\varepsilon)(\Gamma):=\left\{\sigma \mid \exists \Gamma^{\prime} \text { with } d\left(\Gamma, \Gamma^{\prime}\right) \leq \varepsilon \text { such that } \sigma \text { is } \varepsilon \text {-perfect in } \Gamma^{\prime}\right\}
$$

for every $\varepsilon>0$ and every one-person game $\Gamma$.

Proof. Let $\varphi$ be the weakly perfect equilibrium correspondence and $\Phi(\varepsilon)$ the strongly consistent solution generated by $\Phi^{1}(\varepsilon)$. First, we show that $\Phi(\varepsilon)$ is equal to the solution $\Psi(\varepsilon)$ given by

$$
\Psi(\varepsilon)(\Gamma):=\left\{\sigma \mid \exists \Gamma^{\prime} \text { with } d\left(\Gamma, \Gamma^{\prime}\right) \leq \varepsilon \text { such that } \sigma \text { is } \varepsilon \text {-perfect in } \Gamma^{\prime}\right\}
$$

for every game $\Gamma$.

To this purpose, we first prove that $\Psi(\varepsilon)(\Gamma) \subset \Phi(\varepsilon)(\Gamma)$ for each game $\Gamma$.

Let $\sigma \in \Psi(\varepsilon)(\Gamma)$, which means that there is a game $\Gamma^{\prime}$ with $d\left(\Gamma, \Gamma^{\prime}\right) \leq \varepsilon$ such that $\sigma$ is $\varepsilon$-perfect in $\Gamma^{\prime}$. For every $i$, we have that $d\left(\Gamma^{\{i\}, \sigma}, \Gamma^{\prime\{i\}, \sigma}\right) \leq \varepsilon$ and $\sigma_{i}$ is $\varepsilon$-perfect in $\Gamma^{\prime\{i\}, \sigma}$. So, by definition, $\sigma_{i} \in \Phi(\varepsilon)\left(\Gamma^{\{i\}, \sigma}\right)$ for every $i$, which implies that $\sigma \in \Phi(\varepsilon)(\Gamma)$.

Next, we show that $\Phi(\varepsilon)(\Gamma) \subset \Psi(\varepsilon)(\Gamma)$.

Let $\sigma \in \Phi(\varepsilon)(\Gamma)$, so $\sigma_{i} \in \Phi(\varepsilon)\left(\Gamma^{(i), \sigma}\right)$ for every $i$. Hence, for every $i$ we can find a game $\Gamma_{i}^{*}$ with $d\left(\Gamma^{\{i\}, \sigma}, \Gamma_{i}^{*}\right) \leq \varepsilon$ such that $\sigma_{i}$ is $\varepsilon$-perfect in $\Gamma_{i}^{*}$. Let $u_{i}^{*}(z)$ be the payoffs in $\Gamma_{i}^{*}$ at the terminal nodes $z$. We define the game $\Gamma^{\prime}=\left\langle\mathcal{S}, u^{\prime}\right\rangle$ (with the same extensive form structure as $\Gamma$ ) by $u_{i}^{\prime}(z):=u_{i}^{*}(z)$ for every $i$ and every terminal node $z$. Then, by construction, $d\left(\Gamma, \Gamma^{\prime}\right) \leq \varepsilon$ and $\sigma$ is $\varepsilon$-perfect in $\Gamma^{\prime}$ which implies that $\sigma \in \Psi(\varepsilon)(\Gamma)$.

This leads to the conclusion that $\Phi(\varepsilon)=\Psi(\varepsilon)$ for every $\varepsilon$.

Let $\psi$ be the limit consistent solution generated by $\Phi^{1}$. We show that $\varphi=\psi$.

First, we prove $\varphi(\Gamma) \subset \psi(\Gamma)$ for every game $\Gamma$.

Let $\Gamma=\langle\mathcal{S}, u\rangle$ be an extensive form game and $\sigma \in \varphi(\Gamma)$. So, $\sigma$ is a weakly perfect equilibrium in $\Gamma$ supported by some sequences $\Gamma^{k}=\left\langle\mathcal{S}, u^{k}\right\rangle$ and $\sigma^{k}$. For every $k$ we define the number $\varepsilon^{k}$ by

$$
\varepsilon^{k}:=\max \left\{\sigma_{h}^{k}(a) \mid a \notin B_{h}\left(\sigma^{k}, \Gamma^{k}\right)\right\}
$$


if $B_{k}\left(\sigma^{k}, \Gamma^{k}\right) \neq A(h)$. If $B_{h}\left(\sigma^{k}, \Gamma^{k}\right)=A(h)$, we define $\varepsilon^{k}:=\frac{1}{k}$.

By definition of $\varepsilon^{k}, \sigma^{k}$ is $\varepsilon^{k}$-perfect in $\Gamma^{k}$ for every $k$ and $\sigma^{k}$ converges to $\sigma$. It remains to show that $\varepsilon^{k}$ converges to 0 . Let $a$ be an action such that $a \notin B_{h}\left(\sigma^{l}, \Gamma^{l}\right)$ for some $l$. Since $\sigma^{k}$ and $\Gamma^{k}$ are supporting sequences for the weakly perfect equilibrium $\sigma$, we have $\sigma_{h}(a)=0$. So, $\lim _{k \rightarrow \infty} \sigma^{k}(a)=0$ for all $a$ with $a \notin B_{k}\left(\sigma^{l}, \Gamma^{l}\right)$ for some $l$, which implies that $\varepsilon^{k}$ converges to 0 .

For every $k$ we choose a number $l(k)$ such that $\varepsilon^{l(k)} \leq \frac{1}{k}$ and $d\left(\Gamma, \Gamma^{l(k)}\right) \leq \frac{1}{k}$. Then, by construction, $\sigma^{l(k)}$ is $\frac{1}{k}$-perfect in $\Gamma^{l(k)}$ and $d\left(\Gamma, \Gamma^{l(k)}\right) \leq \frac{1}{k}$, which implies that $\sigma^{l(k)} \in \Psi\left(\frac{1}{k}\right)(\Gamma)$ for every $k$. Since $\Psi\left(\frac{1}{k}\right)=\Phi\left(\frac{1}{k}\right)$ and $\sigma^{l(k)}$ converges to $\sigma$, it follows that $\sigma \in \psi(\Gamma)$.

Finally, we show that $\psi(\Gamma) \subset \varphi(\Gamma)$.

Let $\sigma \in \psi(\Gamma)$, which means that there is a sequence $\varepsilon^{k}$ of positive numbers converging to 0 and a sequence $\sigma^{k} \in \Phi\left(\varepsilon^{k}\right)(\Gamma)$ converging to $\sigma$. Since $\Phi\left(\varepsilon^{k}\right)=$ $\Psi\left(\varepsilon^{k}\right)$ it follows that for every $k$ there is a game $\Gamma^{k}$ with $d\left(\Gamma, \Gamma^{k}\right) \leq \varepsilon^{k}$ and $\sigma^{k}$ is $\varepsilon^{k}$-perfect in $\Gamma^{k}$. Since the collection of information sets and the set of actions at each information set is finite, we may assume w.l.o.g. that $B_{h}\left(\sigma^{k}, \Gamma^{k}\right)$ is constant over $k$ for each information set $h$. Now, let $a \notin B_{h}\left(\sigma^{k}, \Gamma^{k}\right)$ for some $k$. Then, by assumption, $a \notin B_{h}\left(\sigma^{k}, \Gamma^{k}\right)$ for all $k$. Since $\sigma^{k}$ is $\varepsilon^{k}$-perfect in $\Gamma^{k}, \sigma_{h}^{k}(a) \leq \varepsilon^{k}$ for every $k$ which implies that $\sigma_{h}(a)=0$. Using the fact that $\Gamma^{k}$ converges to $\Gamma$, we may conclude that $\sigma$ is a weakly perfect equilibrium in $\Gamma$, so $\sigma \in \varphi(\Gamma)$.

Hence the solutions $\varphi$ and $\psi$ are equal, implying that $\varphi$ is the limit consistent solution generated by $\Phi^{1}$.

\subsection{Sequential equilibria}

For the definition of sequential equilibria, we refer to Chapter 2, Section 2.2 .

Theorem 8.8.1 (Kreps and Wilson, 1982) In an extensive form game, a BSP $\sigma$ can be extended to a sequential equilibrium if and only if $\sigma$ is a weakly perfect equilibrium.

We call an assessment $(\sigma, \beta) \varepsilon$-perfect if $\sigma$ is completely mixed, $(\sigma, \beta)$ is Bayesian consistent and $\sigma_{h}(a) \leq \varepsilon$ whenever $a \notin B_{h}(\sigma, \beta, \Gamma)$.

Obviously, for every $\varepsilon$-perfect assessment $(\sigma, \beta)$ it holds that $\sigma$ is an $\varepsilon$-perfect BSP. (As defined in the previous section.)

In the following theorem, we show that every sequential equilibrium can be approximated by a sequence of $\varepsilon$-perfect assessments of perturbed games. 
Theorem 8.8.2 Let $\Gamma=\langle\mathcal{S}, u\rangle$ be an extensive form garne and $(\sigma, \beta)$ a sequential equilibrium in $\Gamma$. Then, there is a sequence $\varepsilon^{k}$ of positive numbers converging to 0 , a sequence $\Gamma^{k}=\left\langle\mathcal{S}, u^{k}\right\rangle$ of games converging to $\Gamma$ and a sequence $\left(\sigma^{k}, \beta^{k}\right)$ of $\varepsilon^{k}$-perfect assessments in $\Gamma^{k}$ converging to $(\sigma, \beta)$.

Proof. Let $(\sigma, \beta)$ be a sequential equilibrium in the game $\Gamma$ with supporting sequence $\left(\sigma^{k}, \beta^{k}\right)$. W.l.o.g. we may assume that $B_{h}\left(\sigma^{k}, \beta^{k}, \Gamma\right)$ is constant over $k$ for every $h$.

The proof consists of two steps. In the first step, we construct a game $\Gamma^{k}$ for every $k$ such that $B_{h}(\sigma, \beta, \Gamma) \subset B_{h}\left(\sigma^{k}, \beta^{k}, \Gamma^{k}\right)$. In the second step, we prove that $\left(\sigma^{k}, \beta^{k}\right)$ is an $\varepsilon^{k}$-perfect assessment in $\Gamma^{k}$ for some $\varepsilon^{k}, \varepsilon^{k}$ converges to 0 and $\Gamma^{k}$ converges to $\Gamma$.

Step 1. Let $k$ be fixed. We define a function $f$ which transforms $\Gamma$ into a new game $f(\Gamma)$. The transformation works as follows.

Let $H^{*}$ be the collection of information sets $h$ for which $B_{h}(\sigma, \beta, \Gamma)$ is not a subset of $B_{h}\left(\sigma^{k}, \beta^{k}, \Gamma\right)$. For every $i$ and every terminal node $z$ we define the payoff $u_{i}^{*}(z)$ in the following way.

If $z$ does not follow any information set in $H_{i}^{*}$, we define $u_{i}^{*}(z):=u_{i}(z)$.

Otherwise, there is exactly one player $i$ information set $h$ in $H^{*}$ which precedes $z$ and is not followed by any other player $i$ information set in $H^{*}$. Since $B_{h}(\sigma, \beta, \Gamma)$ is not a subset of $B_{h}\left(\sigma^{k}, \beta^{k}, \Gamma\right)$, the set $B_{h}(\sigma, \beta, \Gamma) \backslash B_{h}\left(\sigma^{k}, \beta^{k}, \Gamma\right)$ is not empty. Let $b$ be an arbitrary but fixed action in $B_{k}\left(\sigma^{k}, \beta^{k}, \Gamma\right)$. Since $B_{h}\left(\sigma^{k}, \beta^{k}, \Gamma\right)$ is constant over $k$, we can choose $b$ independently of $k$. For every $a \in B_{h}(\sigma, \beta, \Gamma) \backslash B_{h}\left(\sigma^{k}, \beta^{k}, \Gamma\right)$ we define

$$
u_{i}^{*}(z):=u_{i}(z)+\left[U_{i}\left(\sigma^{k} \backslash b, \beta^{k} \mid h\right)-U_{i}\left(\sigma^{k} \backslash a, \beta^{k} \mid h\right)\right] \text { for all } z \in Z(a)
$$

where $Z(a)$ denotes the set of terminal nodes which follow the action $a$. For all other terminal nodes $z$ which follow the information set $h$, we define $u_{i}^{*}(z):=u_{i}(z)$.

In this way, we obtain the game $\left\langle S, u^{*}\right\rangle$ which we call $f(\Gamma)$.

By construction of the payoffs $u^{*}, B_{h}(\sigma, \beta, \Gamma)$ is a subset of $B_{h}\left(\sigma^{k}, \beta^{k}, f(\Gamma)\right)$ for all player $i$ information sets $h$ in $H^{*}$ which are not followed by any other player $i$ information sets in $H^{*}$.

If such a player $i$ information set $h$ in $H^{*}$ is followed by another player $i$ information set $h^{\prime} \notin H^{*}$, there is, by perfect recall, a unique action at $h$ which leads to $h^{\prime}$. By definition of the payoffs $u^{*}$, the set $B_{h^{\prime}}\left(\sigma^{k}, \beta^{k}, \Gamma\right)$ does not change when we go from the game $\Gamma$ to the game $f(\Gamma)$.

Combining the two observations above leads to the conclusion that the set $H^{*}$ becomes strictly smaller when we go from $\Gamma$ to $f(\Gamma)$ (if $H^{*}$ is not empty 
at $\Gamma$, of course) So, by subsequently applying the transformation $f$, the set $H^{*}$ becomes empty after finitely many times.

We define $\Gamma^{k}$ to be the game which we obtain by subsequently applying $f$ until $H^{*}$ is empty. Since $H^{*}$ is empty, it holds that $B_{h}\left(\sigma, \beta_{*} \Gamma\right)$ is a subset of $B_{h}\left(\sigma^{k}, \beta^{k}, \Gamma^{k}\right)$ for all $h$.

Step 2:. For every $k$, we define the number $\varepsilon^{k}$ by

$$
\varepsilon^{k}:=\max \left\{\sigma_{h}^{k}(\alpha) \mid a \notin B_{h}\left(\sigma^{k}, \beta^{k}, \Gamma^{k}\right)\right\}
$$

if $B_{h}\left(\sigma^{k}, \beta^{k}, \Gamma^{k}\right) \neq A(h)$ and $\epsilon^{k}:=\frac{1}{k}$ otherwise. By construction, $\left(\sigma^{k}, \beta^{k}\right)$ is $\varepsilon^{k}$-perfect in $\Gamma^{k}$.

In order to show that $\varepsilon^{k}$ converges to 0 , choose an action a with a $\notin$ $B_{h}\left(\sigma^{k}, \beta^{k}, \Gamma^{k}\right)$ for some $k$. Since $B_{h}\left(\sigma, \beta_{n} \Gamma\right) \subset B_{h}\left(\sigma^{k}, \beta^{k}, \Gamma^{k}\right)$, it follows that $a \notin B_{h}(\sigma, \beta, \Gamma)$. Since $\left(\sigma_{,} \beta\right)$ is sequentially rational, $\sigma_{h}(a)=0$. Hence, $\sigma_{h}^{k}(a)$ converges to 0 , which implies that $\varepsilon^{k}$ converges to 0 .

It remains to show that $\Gamma^{k}$ converges to $\Gamma$. Let $k$ be fixed for the moment. In the step from $\Gamma$ to $f(\Gamma)$, the player $i$ payoff of a terminal node $z$ only changes if $z$ follows a player $i$ action a such that $a \in B_{h}(\sigma, \beta, \Gamma)$ but $a \notin B_{h}\left(\sigma^{k}{ }_{*} \beta^{k}, \Gamma\right)$. If the payoff changes, the new payoff $u_{i}^{*}(z)$ is given by

$$
u_{i}^{*}(z):=u_{i}(z)+\left[U_{i}\left(\sigma^{k} \backslash b, \beta^{k} \mid h\right)-U_{i}\left(\sigma^{k} \backslash a, \beta^{k} \mid h\right)\right]
$$

where $b \in B_{h}\left(\sigma^{k}, \beta^{k}, \Gamma\right)$. Since we assumed that $B_{h}\left(\sigma^{k}, \beta^{k}, \Gamma\right)$ remains constant over $k$, it follows that $b \in B_{h}(\sigma, \beta, \Gamma)$. However, this implies that $U_{i}(\sigma \backslash b, \beta \mid h)=U_{i}(\sigma \backslash a, \beta \mid h)$. Using the fact that $\left(\sigma^{k}, \beta^{k}\right)$ converges to $(\sigma, \beta)$ it follows that

$$
\lim _{k \rightarrow \infty}\left[U_{i}\left(\sigma^{k} \backslash b, \beta^{k} \mid h\right)-U_{i}\left(\sigma^{k}\left|a, \beta^{k}\right| h\right)\right]=0
$$

So, the difference $\left|u_{i}^{\prime \prime}(z)-u_{i}(z)\right|$ tends to zero when $k$ tends to infinity. Since the game $\Gamma^{k}$ is obtained from $\Gamma$ by applying the function $f$ at most $|H|$ times, it follows that $\Gamma^{k}$ converges to $\Gamma$.

This completes the proof.

Theorem 8.8.3 The sequential equilibrium correspondence is a limit consistent assessment solution generated by the one-person solution function $\Phi^{1}$ given by

$\Phi^{1}(\varepsilon)(\Gamma):=\left\{(\sigma, \beta) \| \exists \Gamma^{\prime}\right.$ with $d\left(\Gamma, \Gamma^{\prime}\right) \leq \varepsilon$ such that $(\sigma, \beta)$ is $\varepsilon$-perfect in $\left.\Gamma^{\prime \prime}\right\}$ for every one-person game $\Gamma$ and every $\varepsilon>0$. 
Proof. Let $\varphi$ be the sequential equilibrium correspondence and $\psi$ the limit consistent solution generated by $\Phi^{1}$. Furthermore, we denote by $\Phi(\varepsilon)$ the strongly consistent solution generated by $\Phi^{\mathbb{N}}(\varepsilon)$. Similarly to the proof of Theorem 8.7.1, it can be shown that

$\Phi(\varepsilon)(\Gamma)=\left\{(\sigma, \beta) \mid \exists \Gamma^{\prime}\right.$ with $d\left(\Gamma, \Gamma^{\prime \prime}\right) \leq \varepsilon$ such that $(\sigma, \beta)$ is $\varepsilon$-perfect in $\left.\Gamma^{\prime}\right\}$

for every game $\Gamma$.

Now, we show that $\varphi=\psi$.

First, we prove $\varphi(\Gamma) \subset \psi(\Gamma)$ for every game $\Gamma$.

Let $(\sigma, \beta) \in \varphi(\Gamma)$ for a game $\Gamma$, which means that $(\sigma, \beta)$ is a sequential equilibrium in $\Gamma$. By Theorem 8.8.2 there is a sequence $\varepsilon^{k}$ converging to 0 , a sequence $\Gamma^{k}$ converging to $\Gamma$ and a sequence $\left(\sigma^{k}, \beta^{k}\right)$ of $\varepsilon^{k}$-perfect assessments in $\Gamma^{k}$ converging to $(\sigma, \beta)$. For every $k$ there is an $l(k)$ such that $d\left(\Gamma, \Gamma^{l(k)}\right) \leq \frac{1}{k}$ and $\varepsilon^{l(k)} \leq \frac{1}{k}$. But then, obviously, $\left(\sigma^{l(k)}, \beta^{l(k)}\right) \in \Phi\left(\frac{1}{k}\right)(\Gamma)$ for every $k$. Since $\left(\sigma^{i(k)}, \beta^{l(k)}\right)$ converges to $(\sigma, \beta)$, it follows that $(\sigma, \beta) \in \psi(\Gamma)$.

Finally, we show $\psi(\Gamma) \subset \varphi(\Gamma)$.

Let $(\sigma, \beta) \in \psi(\Gamma)$, so there is a sequence $\varepsilon^{k}$ converging to 0 and a sequence $\left(\sigma^{k}, \beta^{k}\right) \in \Phi\left(\varepsilon^{k}\right)(\Gamma)$ converging to $(\sigma, \beta)$. So, for every $k$ there is a game $\Gamma^{k}$ with $d\left(\Gamma, \Gamma^{k}\right) \leq \varepsilon^{k}$ such that $\left(\sigma^{k}, \beta^{k}\right)$ is $\varepsilon^{k}$-perfect in $\Gamma^{k}$. We show that $(\sigma, \beta)$ is a sequential equilibrium.

The consistency of $(\sigma, \beta)$ follows from the fact that $\left(\sigma^{k}, \beta^{k}\right)$ is a sequence of completely mixed, Bayesian assessments converging to $(\sigma, \beta)$.

It remains to show that $(\sigma, \beta)$ is sequentially rational. To this purpose, consider an information set $h$ and an action $a \in A(h)$ such that $a \notin B_{h}(\sigma, \beta, \Gamma)$. Since we may assume that $B_{k}\left(\sigma^{k}, \beta^{k}, \Gamma^{k}\right)$ remains constant over $k$, it follows that $a \notin B_{h}\left(\sigma^{k}, \beta^{k}, \Gamma^{k}\right)$ for every $k$. Using the fact that $\left(\sigma^{k}, \beta^{k}\right)$ is $\varepsilon^{k}$-perfect in $\Gamma^{k}$, it holds that $\sigma_{h}^{k}(a) \leq \varepsilon^{k}$ for every $k$ implying that $\sigma_{h}(a)=0$. It follows that $(\sigma, \beta)$ is sequentially rational. 


\section{Chapter 9}

\section{Non-cooperative games with costly coordination}

\subsection{Introduction}

This chapter is based on Perea y Monsurwe (1997b).

In this chapter, we consider non-cooperative situations in which players have the possibility to coordinate their actions, without being able to make binding agreements about the strategies that will be played. For such situations, the Nash equilibrium condition is a necessary condition for self-enforcability, but, in general, not sufficient. Starting from a Nash-equilibrium, a coalition of players may have the incentive to coordinate their actions and deviate collectively from the agreed upon strategy profile. In order to overcome this problem, Aumann (1959) defined the notion of strong Nash equilibria, i.e. strategy profiles in which no coalition of players has an incentive to deviate. Implicit in this concept is the assumption that coalitions can make binding agreements about the deviation that will be played.

Later, Bernheim et al. (1987) modified the concept by considering only selfenforcing deviations by coalitions. The resulting concept, which is a coarsening of strong Nash equilibrium, is called coalition-proof Nash equilibrium. In recent years, the idea of coalition-proofness has been applied to the case where players can not only coordinate their actions but also have the possibility to correlate their play. For further reading on this topic, we refer to Ray (1996), Moreno and Wooders (1996) and Milgrom and Roberts (1996). In all the above mentioned papers, coordination between players is assumed to be costless. However, in many practical situations, costly facilities are required to make coordination between players possible. Think, for instance, of communication networks between players. Each time the players of a 
coalition want to coordinate their actions, they have to use the network and must pay the corresponding costs.

The aim of this chapter is to describe such situations with costly coordination and to develop meaningful solution concepts for this case. Formally, we model a non-cooperative situation with costly coordination by a normal form game and a cooperative game assigning to every coalition of players the cost required for coordination between its members. The pair obtained in this way is called a game with costly coordination.

In this model we assume that, once a coalition has decided to coordinate the actions, the members can make binding agreements about the deviation that will be chosen and the way in which the coordinations costs and deviation payoffs are shared. Within this context, we present a self-enforcing solution concept selecting those strategy profiles where no coalition of players has an incentive to deviate. A key role in this concept is played by the socalled excess of a coalition which reflects the maximal improvement that can be achieved if the coalition decides to coordinate the actions, pay the coordination costs and deviate collectively to some other strategy profile. Hence, the excess depends crucially on the agreed upon strategy profile and can be viewea as a tool which measures the willingness of a coalition to deviate from this strategy profile. The equilibria selected by the concept are exactly those equilibria at which all ecesses are non-positive. These equilibria are called negative excess equilibria (NE-equilibria). In the extreme case where all coordination costs are zero, every NE-equilibrium is a strong Nash equilibrium. In the other extreme, where coordination costs are very high, the NE-equilibria coincide with the Nash equilibria.

Next, we consider a non-cooperative, dynamic implementation of the process of coordination and collective deviation. Starting from an agreed upon strategy profile, we define an extensive form game, called the local deviation game, which can last infinitely many periods. In every period, players have the possibility to 'deviate' or to 'not deviate'. However, each player can only deviate once during the play of the game. If, in a certain period, a given coalition of players decides to 'deviate', the members will coordinate their actions, deviate collectively and share the net deviation payoff according to a given mechanism. By net deviation payoff, we mean the aggregate payoff that the coalition can obtain by deviating collectively minus the coordination costs. Afterwards, the original payoffs of the coalition members will be replaced by these 'new' payoffs. Within this setting, we give several characterizations of NE-equilibria by means of non-cooperative solutions for the local deviation game. For instance, the set of NE-equilibria consists exactly of those strategy profiles for which 'never deviate' is a dominant strategy in the corresponding local deviation game. 
A common feature of all these characterizations is the fact that they make use of the strategy profile where all players choose the strategy 'never deviate'. In the next section, we concentrate on the situation where all players in the local deviation game choose the strategy 'always deviate'. It turns out that the non-cooperative properties of this strategy profile are closely related to cooperative properties of the excess vector, which can be viewed as a cooperative game. Among other results, we show that the strategy profile 'everybody always deviate' is a NE-equilibrium in the local deviation game if and only if the corresponding excess vector is balanced.

A disadvantage of NE-equilibria is the fact that their existence is not guaranteed. Therefore, it seems useful to develop a solution concept which covers, as much as possible, the ideas of NE-equilibria and has the property that the solution is always non-empty. We present a possible way to do this by introducing so-called minimal excess solutions (ME-solutions). A minimal excess solution selects those strategy profiles where the corresponding excess vector, consisting of the excesses of all possible coalitions, is minimal with respect to some relation on the space of excess vectors. This implies that the solution depends crucially on the relation that has been chosen. The main idea behind this concept is the observation that an excess vector reflects the willingness of every coalition to deviate from the agreed upon strategy profile. Strategy profiles with minimal excess vectors can therefore be seen as those profiles which are maximally stable against coalitional deviations. However, there is no unique way to define minimality of excess vectors since excess vectors are multi-dimensional entities. The most general way to do this is to define minimality with respect to some relation. Under very mild conditions on the relation, we prove that the corresponding ME-solution is always non-empty.

One could choose, for instance, the lexicographic relation resulting in a solution which we call the lexicographic ME-solution. This solution is very similar to the nucleolus for cooperative games since it selects, roughly speaking, those strategy profiles where the maximal excess is minimal. Another interesting class of ME-solutions consists of the so-called linear ME-solutions, induced by linear relations.

Finally, we characterize ME-solutions by means of non-cooperative solutions in the local deviation game. In these characerizations, we make use of the above mentioned characterizations of NE-equilibria.

The layout of the chapter is as follows. In section 2, we introduce games with costly coordination whereas section 3 presents negative excess solutions. A dynamic implementation of coordination by means of local deviation games is given in section 4 . We characterize NE-solutions by solutions of local deviation games in section 5 . Section 6 analyzes the relationship between 
non-cooperative properties of the strategy profile 'everybody always deviate' in the local devation game and the cooperative properties of the corresponding excess vector. In section 7 , we introduce minimal excess solutions and prove their existence under standard assumptions, whereas section 8 gives some examples for minimal excess solutions. We conclude by characterizing minimal excess solutions by means of local deviation games in section 9 .

\subsection{Games with costly coordination}

In this section, we formally introduce normal form games with costly coordination. Before doing so, we briefly describe normal form games and TU-games.

\section{Normal form games and TU-games}

A normal form game is a pair $\Gamma=\langle M, v\rangle$ where $M=\prod_{i \in N} M_{i}$ is the finite product of finite pure strategy spaces $M_{i}$ and $v$ is a payoff function which assigns to every pure strategy profile in $M$ a payoff-vector in $\mathbb{R}^{N}$.

A mixed strategy for player $i$ is a probability distribution $p_{i}$ on $M_{i}$. A vector $p=\left(p_{i}\right)_{i \in N}$ consisting of mixed strategies is called a mixed strategy profile (MSP).

By $\nu_{i}(p)$ we denote the expected payoff for player $i$ if the MSP $p$ is played.

A $T U$-game is a pair $\langle N, \bar{v}\rangle$ where $N$ is a finite set of players and $\bar{v}$ is a function which assigns to every coalition $S \subset N$ some value $\bar{v}(S)$. We assume that $\bar{v}(\varnothing)=0$.

If there can be no misunderstanding about the player set $N$, we write $\bar{v}$ instead of $\langle N, \bar{v}\rangle$.

The restriction of $\tilde{v}$ on a subcoalition of $N$ is called a subgame.

\section{Games with costly coordination}

We consider a situation in which the players of a normal form game have the possibility to coordinate their actions, without being able to make binding agreements about the orginal actions to be played. However, the players can make binding agreements about deviations from the orginal action profile. We assume that coordination between players requires certain costs. Think, for instance, of costs which have to be made in order to make communication 
between the players possible. We model this situation by assurning that every coalition $S$ of players has a certain cost $c(S) \geq 0$ which is the cost required for coordinating the actions between the players in $S$. Hence, the cost vector $c$ is in fact a non-negative TU-game with player set $N$.

We assume that coordination of the actions of a single player is costless, so $c(\{i\})=0$ for all $i \in N$. We say that $c$ is zero-normalized.

A pair $\langle\Gamma, c\rangle$ consisting of a normal form game $\Gamma$ and a cost vector $c$ is called a game with costly coordination.

\subsection{Negative excess solutions}

\section{Excesses}

For a MSP $p$ we want to measure the willingness of a coalition of players to coordinate their actions and deviate collectively from $p$. This willingness can be reflected by the so-called 'excess' of the coalition at the MSP $p$, which is the maximal profit that the players in the coalition can obtain together by deviating collectively.

Formally, for an MSP $p$ and a coalition $S$ of players we define the excess $e(p, S)$ by

$$
e(p, S)=\max _{m_{S}} \sum_{i \in S} v_{i}\left(p \backslash m_{S}\right)-c(S)-\sum_{i \in S} v_{i}(p) .
$$

Here, $m_{S}$ denotes a pure strategy profile for the players in $S$ and by $p \backslash m_{S}$ we mean the strategy profile where players in $S$ play according to $m_{S}$ and all other players play acoording to $p$.

The excess vector $e(p) \in \mathbb{R}^{2^{N}}$ is defined by

$$
e(p)=(e(p, S))_{S \in 2^{N}} .
$$

\section{Negative excess solutions}

The excess vector of an MSP can be used as a local criterion to decide whether a coalition of players is willing to coordinate their actions or not. If the excess of the coalition is greater than zero, the players in the coalition can coordinate their actions, deviate collectively and divide the total net payoff such that every player gets at least his original payoff $v_{i}(p)$. In this case, it is optimal for the coalition to coordinate the actions and deviate collectively. If the excess of coalition $S$ is less or equal than zero, there is no collective 
deviation of the members of $S$ that will give each player at least his original payoff and make one or more players strictly better off. In this case, coordination and collective deviation is not profitable for the coalition at the given MSP. The MSP's at which all excesses are less or equal to zero are therefore exactly the MSP's which are stable against collective deviation of every coalition. These MSP's are called negative excess equilibria.

The solution which assigns to every game with costly coordination the set of NE-equilibria is called the negative excess solution.

Obviously, every NE-equilibrium is a Nash equilibrium in the normal form game since in a NE-equilibrium all the excesses of one-person coalitions are zero which implies that unilateral deviation (against cost zero) is not profitable.

If the costs of coordination are high enough, the NE-equilibria coincide exactly with the Nash equilibria since in this case the excesses for coalitions with more than one player are always negative.

If the costs of coordination are all equal to zero, it can be shown that every NE-equilibrium is a strong Nash equilibrium (Aumann, 1959) in the normal form game.

Theorem 9.3.1 Let $\langle\Gamma, c\rangle$ be game with costly coordination in which all $\operatorname{costs} c(S)$ are equal to zero. Then, every $N E$-equilibrium of $\langle\Gamma, c\rangle$ is a strong Nash equilibrium in $\Gamma$.

Proof. Let $p$ be a NE-equilibrium in $\langle\Gamma, c\rangle$. Suppose that $p$ is not a strong Nash equilibrium in $\Gamma$, which means that there is a coalition $S$ and a pure strategy profile $m_{S}$ for coalition $S$ such that $v_{i}\left(p \backslash m_{S}\right)>v_{i}(p)$ for all $i \in S$. Then,

$$
\sum_{i \in S} v_{i}\left(p \backslash m_{S}\right)>\sum_{i \in S} v_{i}(p)
$$

which implies that $e(p, S)>0$. This is a contradiction to our assumption that $p$ is a NE-equilibrium.

Since we know that some normal form games have no strong Nash equilibrium, it follows that the existence of NE-equilibria is not guaranteed in general.

Example 9.1. Consider a game with costly coordination $\langle\Gamma, c\rangle$ in which $\Gamma$ is the prisoner's dilemma given by

$$
\left[\begin{array}{ll}
1,1 & 3,0 \\
0,3 & 2,2
\end{array}\right]
$$


The pure strategies for player 1 are $T$ (Top) and $B$ (Bottom) whereas player 2 has the pure strategies $L$ (Left) and $R$ (Right).

For every MSP $p=(\lambda T+(1-\lambda) B, \mu L+(1-\mu) R)$ the excess vector $e(p)$ is given by

\begin{tabular}{|r|l|}
\hline 1 & $1-\lambda$ \\
\hline 2 & $1-\mu$ \\
\hline 12 & $\lambda+\mu-c(12)$ \\
\hline
\end{tabular}

Here, the coalitions are written at the left hand side of the table whereas the corresponding excesses are written on the right hand side.

It holds that $e(p, 1)+e(p, 2)+e(p, 12)=2-c(12)$ which implies that there is no NE-equilibrium in this game with costly coordination if $c(12)<2$.

If $c(12) \geq 2$, then $e(p) \leq 0$ if and only if $\lambda=\mu=1$ which implies that $(T, L)$ is the unique NE-equilibrium in this case.

Example 9.2. Let $\langle\Gamma, c\rangle$ be a game with costly coordination in which $\Gamma$ is equal to the three person game given by

$$
\left[\begin{array}{ll}
1,1,0 & 3,0,0 \\
0,3,0 & 2,2,0
\end{array}\right] \quad\left[\begin{array}{ll}
0,0,0 & 0,0,0 \\
0,0,0 & 0,0,0
\end{array}\right]
$$

The pure strategies for player 1 and 2 are $T, B$ and $L, R$ respectively, whereas player 3 can choose between $W$ (West) and $E$ (East). The first and the second matrix denote the situations where player 3 chooses $W$ and $E$ respectively. Now, let the MSP $p$ be given by

$$
p=(\lambda T+(1-\lambda) B, \mu L+(1-\mu) R, \nu W+(1-\nu) E) .
$$

The corresponding excess vector $e(p)$ is given by

\begin{tabular}{|r|l|}
\hline 1 & $\nu(1-\lambda)$ \\
\hline 2 & $\nu(1-\mu)$ \\
\hline 3 & 0 \\
\hline 12 & $\nu(\lambda+\mu)-c(12)$ \\
\hline 13 & $\nu(1-\lambda)+(1-\nu)(3-2 \mu)-c(13)$ \\
\hline 23 & $\nu(1-\mu)+(1-\nu)(3-2 \lambda)-c(23)$ \\
\hline 123 & $\nu(\lambda+\mu)+(1-\nu) 4-c(123)$ \\
\hline
\end{tabular}

(a) Let $c$ be given by $c(S)=\frac{1}{2}$ if $|S|=2$ and $c(123)=2$.

The sum of all excesses is equal to

$$
\sum_{S} e(p, S)=\nu 4+(1-\nu)(10-2 \lambda-2 \mu)-3 \frac{1}{2}>0
$$


since $10-2 \lambda-2 \mu \geq 4$. Therefore, we may conclude that the game with costly coordination has no $\mathrm{NE}$-equilibria.

(b) Now, choose $c(12)=2, c(13)=c(23)=0$ and $c(123)=2$.

In this case, the sum of all excesses is equal to

$$
\sum_{S} e(p, S)=\nu 4+(1-\nu)(10-2 \lambda-2 \mu)-4
$$

which is less or equal to zero only if $\nu=1$. Hence, if there is a NE-equilibrium, then player 3 must choose $\nu=1$. Since $e(p, 1)$ and $e(p, 2)$ must be less or equal to zero, it follows that $\lambda=\mu=1$ must hold in a NE-equilibrium. As can be seen easily, $\lambda=\mu=\nu=1$ constitutes a NE-equilibrium. Therefore, $(T, L, W)$ is the unique NE-equilibrium in this game with costly coordination.

\subsection{Dynamic implementation of coordination}

In this section, we introduce a dynamic non-cooperative setting for the process of coordination and collective deviation by defining for every MSP an extensive form game which we will call the local deviation game. In this game, which can last for an infinite number of periods, every player has the ability to deviate once from the proposed MSP $p$. However, each player can choose the period in which he wants to deviate. In every period, the coalition of players who decide to deviate in this period coordinate their actions, pay the corresponding cost of coordination and deviate collectively to a strategy profile which maximizes the total payoff of the coalition. Afterwards, the players in $S$ distribute the total net payoff among the members of the coalition according to a given allocation mechanism. In the next section, we show that the $\mathrm{NE}$-equilibria of the original game with costly coordination can be characterized in several ways by non-cooperative solutions of this local deviation game.

\section{The local deviation game}

Let $p$ be a MSP proposed by the players of the game with costly coordination. An allocation mechanism for $p$ is a function $x$ which assigns to every coalition $S$ of players a vector $x(S)=\left(x_{i}(S)\right)_{i \in S}$ which distributes the payoff

$$
\bar{v}(p, S):=\max _{m_{S}} \sum_{i \in S} v_{i}\left(p \backslash m_{S}\right)-c(S)
$$


among the members of $S$.

For a MSP $p$ and an allocation mechanism $x$, the local deviation game $\Gamma(p, x)$ is played as follows.

At the beginning of the game, each player has his original payoff $v_{i}(p)$. In period 1, every player has two pure actions: 'deviate' and 'not deviate'. If $S$ is the coalition of players who choose 'deviate', the players in $S$ coordinate their actions at cost $c(S)$, deviate collectively to a strategy profile which maximizes the total payoff of $S$, distribute the net payoff $v(p, S)$ among the members of $S$ according to $x(S)$ and replace their original payoff $v_{i}(p)$ by the new payoff $x_{i}(S)$. At this point, the game is over for the players in $S$.

In period 2, the remaining players in $N \backslash S$ can choose between 'deviate' and 'not deviate'. If $T \subset N \backslash S$ is the coalition of players who choose 'deviate', they coordinate their actions at $\operatorname{cost} c(T)$, deviate collectively to a strategy profile which maximizes the payoff of $T$ and divide the net payoff $\bar{v}(p, T)$ among the members of $T$ according to $x(T)$. At this point, the game is over for the players in $T$. And so on.

If a player never decides to 'deviate", he receives his original individual payoff $v_{i}(p)$.

If the players choose a behavior strategy profile (BSP) $\left(\sigma_{i}\right)_{i \in N}$, the expected payoff for each player $i$ in the local deviation game is denoted by $u_{i}(\sigma)$.

Non-cooperative solutions for the local deviation game

We briefly introduce some non-cooperative solutions for the local deviation game which are used in the following two sections.

A subgame perfect equilibrium is a BSP which constitutes a Nash equilibrium in every subgame of $\Gamma(p, x)$.

Note that every subgame which starts in period $n$ can be identified with a history $S_{1}, S_{2}, \ldots, S_{n-1}$ where $S_{k}$ is the coalition of players who have 'deviated' in period $k$.

For a BSP $\sigma=\left(\sigma_{i}\right)_{i \in N}$ and a coalition $S$ we define the excess

$$
e(\sigma, S)=\sup _{\tau_{S}} \sum_{i \in S} u_{i}\left(\sigma \backslash \tau_{S}\right)-\sum_{i \in S} u_{i}(\sigma)
$$

where $\tau_{S}$ is a pure strategy profile for the coalition $S$. In the definition of the excess, we assume that the coordination costs in the local deviation game is zero. However, the coordination costs of the game with costly coordination are present in the payoffs of the local deviation game. 
A negative excess equilibrium (NE-equilibrium) in the local deviation game is a BSP $\sigma$ for which the excesses $e(\sigma, S)$ of all coalitions are less or equal then zero.

A BSP $\sigma$ is called a subgame perfect NE-equilibrium if it constitutes a NEequilibrium in every subgame of $\Gamma(p, x)$.

A pure strategy for player $i$ in the local deviation game is called dominant if it is a best response against every BSP of the other players.

A BSP $\sigma$ is called a strong Nosh equilibrium if for every coalition $S$ there is no pure strategy profile $\tau_{S}$ for $S$ such that $u_{i}\left(\sigma \backslash \tau_{S}\right)>u_{i}(\sigma)$ for all $i \in S$.

\subsection{Characterization of NE-equilibria by lo- cal deviation games}

In this section, we give several characterizations of the Nash equilibria and the NE-equilibria of a game with costly coordination by means of the corresponding local deviation game. By Nash equilibria of a game with costly coordination $\langle\Gamma, c\rangle$ we simply mean the Nash equilibria of $\Gamma$. A common feature of these characterizations is the fact that they concentrate on the strategy profile in the local deviation game where every player chooses 'not deviate' at each of his information sets. We call this strategy profile 'everybody never deviate'. The theorems show that there is a close connection between the Nash equilibria and NE-equilibria of the game with costly coordination on one side and the non-cooperative stability of "everybady never deviate' in the corresponding local deviation game on the other side.

Theorem 9.5.1 Let $\langle\Gamma, c\rangle$ be a game with costly coordination, $p$ a MSP and $x$ an allocation mechanism for $p$. Then, $p$ is a Nash equilibrium in $\langle\Gamma, c\rangle$ if and only if 'everybody never deviate' is a Nash equilibrium in $\Gamma(p, x)$.

Proof. (a) Let $p$ be a Nash equilibrium in $\langle\Gamma, c\rangle$. In the BSP 'everybody never deviate', every player gets $v_{i}(p)$. If a player $i$ deviates unilaterally from his behavior strategy 'never deviate', he can obtain at most $\vec{v}(p,\{i\})=\max _{m_{i}} v_{i}\left(p \backslash m_{i}\right)$ which is equal to $v_{i}(p)$ since $p$ is a Nash equilibrium in $\langle\Gamma, c\rangle$. So, no player can benefit from unilateral deviation, which implies that 'everybody never deviate' is a Nash equilibrium.

(b) Let "everybody never deviate" be a Nash equilibrium. If player $i$ deviates from his strategy in $\Gamma(p, x)$ by choosing 'deviate' in period 1 , he gets 
$\bar{v}(p,\{i\})=\max _{m_{i}} v_{i}\left(p \backslash m_{i}\right)$ which should be less or equal then $v_{i}(p)$, the payoff he gets in 'everybody never deviate'. Since this should hold for every player $i, p$ is a Nash equilibrium in $\langle\Gamma, c\rangle$.

It can be easily seen that 'everybody never deviate' is a Nash equilibrium in the local deviation game if and only if it is a subgame perfect equilibrium. Therefore, we obtain the following alternative characterization of Nash equilibria of the game with costly coordination by means of the local deviation game.

Theorem 9.5.2 Let $\langle\Gamma, c\rangle$ be a game with costly coordination, $p$ a MSP and $x$ an allocation mechanism for $p$. Then, $p$ is a Nash equilibrium in $\langle\Gamma, c\rangle$ if and only if 'everybody never deviate' is a subgame perfect equilibrium in $\Gamma(p, x)$.

The NE-equilibria of a game with costly coordination can be characterized by the local deviation game in the following ways.

Theorem 9.5.3 Let $\langle\Gamma, c\rangle$ be a game with costly coordination, $p$ a MSP and $x$ an allocation mechanism for $p$. Then, $p$ is a NE-equilibrium in $\langle\Gamma, c\rangle$ if and only if 'everybody never deviate' is a NE-equilibrium in $\Gamma(p, x)$.

Proof. (a) Let $p$ be a NE-equilibrium in $\langle\Gamma, c\rangle$. In order to show that $\sigma:=$ 'everybody never deviate' is a NE-equilibrium in $\Gamma(p, x)$, it suffices to show that for every coalition $S$ and every pure strategy profile $\tau_{S}$ we have

$$
\sum_{i \in S} u_{i}\left(\sigma \backslash \tau_{S}\right) \leq \sum_{i \in S} u_{i}(\sigma)
$$

Choose a coalition $S$ and an arbitrary pure strategy profile $\tau_{S}$. For every period $n$, let $T_{n} \subset S$ be the coalition of players who 'deviate' in period $n$ on the unique equilibrium path of $\sigma \backslash T_{S}$. Furthermore, let $T_{0} \subset S$ be the coalition of players in $S$ who do 'not deviate' on the equilibrium path of $\sigma \backslash \tau_{S}$. Then, we have

$$
\begin{aligned}
\sum_{i \in S} u_{i}\left(\sigma \backslash \tau_{S}\right) & =\sum_{n} \sum_{i \in T_{n}} x_{i}\left(T_{n}\right)+\sum_{i \in T_{0}} v_{i}(p) \\
& =\sum_{n} \bar{v}\left(p, T_{n}\right)+\sum_{i \in T_{0}} v_{i}(p) \\
& =\sum_{n}\left[\max _{m_{T_{n}}} \sum_{i \in T_{n}} v_{i}\left(p \backslash m_{T_{n}}\right)-c\left(T_{n}\right)\right]+\sum_{i \in T_{0}} v_{i}(p) \\
& \leq \sum_{n} \sum_{i \in T_{n}} v_{i}(p)+\sum_{i \in T_{0}} v_{i}(p) \\
& =\sum_{i \in S} v_{i}(p)=\sum_{i \in S} u_{i}(\sigma) .
\end{aligned}
$$


Here, the inequality follows from the fact that $p$ is a NE-equilibrium in $\langle\Gamma, c\rangle$. (b) Let $\sigma:=$ 'everybody never deviate' be a NE-equilibrium in $\Gamma(p, x)$. Suppose that there is a coalition $S$ with $e(p, S)>0$ which means that $\bar{v}(S)>$ $\sum_{i \in S} v_{i}(p)$. If coalition $S$ deviates collectively from $\sigma$ by choosing 'deviate' in period 1 , they get $\bar{v}(S)$ together, which is strictly more than $\sum_{i \in S} v_{i}(p)$, the total payoff for $S$ when playing $\sigma$. However, this is a contradiction, since 'everybody never deviate' is a NE-equilibrium. Hence, $e(p, S) \leq 0$ for every $S$ which implies that $p$ is a NE-equilibrium in $\langle\Gamma, c\rangle$.

As can be seen easily, 'everybody never deviate' is a NE-equilibrium in the local deviation game if and only if it is a subgame perfect NE-equilibrium. This leads to the following result.

Theorem 9.5.4 Let $\langle\Gamma, c\rangle$ be a game with costly coordination, $p$ a MSP and $x$ an allocation mechanism for $p$. Then, $p$ is a NE-equilibrium in $\langle\Gamma, c\rangle$ if and only if 'everybody never deviate' is a subgame perfect NE-equilibrium in $\Gamma(p, x)$.

Let $p$ be a MSP and $x$ an allocation mechanism for $p$. We say that $x$ is one sided if

$$
\begin{gathered}
\bar{v}(p, S)<\sum_{i \in S} v_{i}(p) \text { implies } x_{i}(S)<v_{i}(p) \text { for all } i \in S, \\
\bar{v}(p, S)=\sum_{i \in S} v_{i}(p) \text { implies } x_{i}(S)=v_{i}(p) \text { for all } i \in S
\end{gathered}
$$

and

$$
\bar{v}(p, S)>\sum_{i \in S} v_{i}(p) \text { implies } x_{i}(S)>v_{i}(p) \text { for all } i \in S .
$$

Intuitively, this means that in case of excess demand $\left(\bar{v}(p, S)<\sum_{i \in S} v_{i}(p)\right)$ every player gets less than his reservation payoff $v_{i}(p)$ and in case of excess supply $\left(\tilde{v}(p, S)>\sum_{i \in S} v_{i}(p)\right)$ every player gets more than his reservation payoff. If $\bar{v}(p, S)=\sum_{i \in S} v_{i}(p)$, every player gets exactly his reservation payoff.

Theorem 9.5.5 Let $\langle\Gamma, c\rangle$ be a game with costly coordination, $p$ a MSP and $x$ a one sided allocation mechanism for $p$. Then, $p$ is a NE-equilibrium in $\langle\Gamma, c\rangle$ if and only if 'everybody never deviate' is a strong Nash equilibrium in $\Gamma(p, x)$. 
Proof. (a) Let $p$ be a NE-equilibrium in $\langle\Gamma, c\rangle$. Then, by Theorem 9.5 .3 , "everybody never deviate' is a NE-equilibrium in $\Gamma(p, x)$. Since the coordination costs in $\Gamma(p, x)$ are zero, it follows that 'everybody never deviate' is a strong Nash equilibrium.

(b) Let $\sigma:=$ "everybody never deviate" be a strong Nash equilibrium in $\Gamma(p, x)$.

Suppose that there is a coalition $S$ with $e(p, S)>0$ which means that $\bar{v}(p, S)>\sum_{i \in S} v_{i}(p)$.

Let $\tau_{S}$ be a pure strategy profile for $S$ in which every member of $S$ chooses 'deviate' in the first period. Then, every player $i \in S$ gets $x_{i}(S)$ in the BSP $\sigma \backslash \tau_{S}$, wheras he obtains $v_{i}(p)$ in the original BSP $\sigma$.

Since $\bar{v}(p, S)>\sum_{i \in S} v_{i}(p)$ and $x$ is one sided, it follows that $x_{i}(S)>v_{i}(p)$ for all $i \in S$. However, this implies that $\sigma$ is not a strong Nash equilibrium, which is a contradiction. Therefore, $e(p, S) \leq 0$ for every $S$, so $p$ is a NE-equilibrium in $\langle\Gamma, c\rangle$.

Theorem 9.5.6 Let $\langle\Gamma, c\rangle$ be a game with costly coordination, $p$ a MSP and $x$ a one sided allocation mechanism for $p$. Then, $p$ is a NE-equilibrium in $\langle\Gamma, c\rangle$ if and only if 'never deviate' is a dominant strategy for every player in $\Gamma(p, x)$.

Proof. (a) Let $p$ be at NE-equilibrium in $\langle\Gamma, c\rangle$. Let $i$ be a player and $\sigma_{N \backslash\{i\}}$ be an arbitrary BSP for the other players. If player $i$ chooses 'never deviate', he gets $v_{i}(p)$. By choosing another pure strategy, he gets at most $\max _{\mathcal{F}_{i} \in S} x_{i}(S)$. Since $e(p, S) \leq 0$ for all $S$ it follows that $\bar{v}(p, S) \leq \sum_{i \in S} v_{i}(p)$ for all $S$. By one sidedness of $x$, we obtain $x_{i}(S) \leq v_{i}(p)$ for all $S$. Therefore, we may conclude that player $i$ can not get more than $v_{i}(p)$ by choosing another pure strategy, which implies that 'never deviate' is a best response. Since this holds for every $i$ and every BSP $\sigma_{N \backslash\{i}$, it follows that 'never deviate' is a dominant strategy for every player.

(b) Let 'never deviate' be a dominant strategy for every player in $\Gamma(p, x)$. Suppose that $e(p, S)>0$ for some coalition $S$, which means that $\tilde{v}(p, S)>$ $\sum_{i \in S} v_{i}(p)$. Since $x$ is one sided, $x_{i}(S)>v_{i}(p)$ for all $i \in S$.

Now, let $i \in S$ be fixed and $\tau_{N \backslash\{i\}}$ be a pure strategy profile of the other players in which players in $S \backslash\{i\}$ 'deviate' in period 1 and players outside $S$ 'never deviate'. By choosing 'never deviate', player $i$ gets $v_{i}(p)$. However, he can get $x_{i}(S)>v_{i}(p)$ by choosing 'deviate' in period 1. Hence, 'never deviate' is not a dominant stratagy for player $i$, which is a contradiction. Therefore, $e(p, S) \leq 0$ for every coalition $S$ which implies that $p$ is a NEequilibrium in $\langle\Gamma, c\rangle$. 


\subsection{Cooperative excess game versus local de- viation game}

For a given MSP $p$, the excess vector $e(p)$ can be interpreted as a TU-game, since it assigns to every coalition $S$ of players the value $e(p, S)$ which is the excess of coalition $S$ at $p$. We can therefore speak about the cooperative excess game $e(p)$. In this section, we show that there is a close connection between cooperative properties of the excess game $e(p)$, such as non-emptiness of imputation sets and balancedness, on one side and non-cooperative solutions of the local deviation game on the other side. In contrast to the previous section, we concentrate now on the strategy profile in the local deviation game in which every player chooses "deviate" at each of his information sets. We call this strategy profile 'everybody always deviate'.

Before stating the theorems, we need some definitions.

Let $\tilde{v}$ be a TU-game on the player set $N$. An imputation of $\bar{v}$ is a vector $y$ with $\sum_{i \in N} y_{i}=\bar{v}(N)$ and $y_{i} \geq \bar{v}(\{i\})$ for all $i \in N$.

For a coalition $S$, we denote the subgame of $\bar{v}$ on $S$ by $\left.\bar{v}\right|_{S}$.

In a game $\langle\Gamma, c\rangle$ with costly coordination, $\bar{v}(p)$ denotes the cooperative game on $N$ assigning to every coalition $S$ the value

$$
\bar{v}(p, S)=\max _{\pi^{2} S} \sum_{i \in S} v_{i}\left(p \backslash m_{S}\right)-c(S)
$$

Theorem 9.6.1 Let $\langle\Gamma, c\rangle$ be a game with costly coordination, $p$ a MSP and $x$ an allocation mechanism for $p$. Then, "everybody always deviate is a Nash equilibrium in $\Gamma(p, x)$ if and only if $x(N)$ is an imputation of the TU.game $\tilde{v}(p)$.

Proof. (a) Let 'everybody always deviate' be a Nash equilibrium in $\Gamma(p, x)$. Suppose that all players except $i$ play 'always deviate'. If player $i$ chooses 'always deviate", he gets $x_{i}(N)$.

If he deviates to another pure strategy, he gets at $\operatorname{most} \max \left\{x_{i}(N), \bar{v}(p,\{i\})\right\}$. Since "everybody always deviate" is a Nash equilibrium, it should hold that $x_{i}(N) \geq \max \left\{x_{i}(N), v(p,\{i\})\right\}$, which means that $x_{i}(N) \geq \bar{v}(p,\{i\})$.

This this should hold for every $i \in N$, it follows that $x(N)$ is an imputation of $w(p)$.

(b) Let $x(N)$ be an imputation of $\bar{v}(p)$ which means that $x_{i}(N) \geq \bar{v}(p,\{i\})$ for every $i \in N$. Suppose that $i$ is a player and all players except $i$ play 'always deviate'. If $i$ chooses 'always deviate', he gets $x_{i}(N)$, whereas other pure strategies give him at most $\max \left\{x_{i}(N), \bar{v}(p,\{i\})\right\}$ which is equal to 
$x_{i}(N)$. Since this holds for every $i \in N$, "everybody always deviate" is a Nash equilibrium.

Theorem 9.6.2 Let $\langle\Gamma$, $c\rangle$ be a game with costly coordination, $p$ a MSP and $x$ an allocation mechanism for $p$. Then, 'everybody always deviate' is a subgame perfect equilibrium in $\Gamma(p, x)$ if and only if $x(S)$ is an imputation of the subgame $\left.\vec{v}(p)\right|_{S}$ for all coalitions $S$.

Proof. (a) Let "everybody always deviate" be a subgame perfect equilibrium in $\Gamma(p, x)$. Let $S$ be an arbitrary coalition and $i \in S$. Let $\Gamma^{*}$ be a subgame in which the players in $N \backslash S$ have already deviated in previous periods and players in $S$ have not.

Suppose that all players except $i$ play 'always deviate' in $\Gamma^{*}$. If player $i$ chooses "always deviate" in $\Gamma^{*}$, he gets $x_{i}(S)$.

If he deviates to another pure strategy in $\Gamma^{*}$, he gets at most

$\max \left\{x_{i}(S), \bar{v}(p,\{i\})\right\}$.

Since 'everybody always deviate' contitutes a Nash equilibrium in $\Gamma^{*}$, it should hold that $x_{i}(S) \geq \max \left\{x_{i}(S), \tilde{v}(p,\{i\})\right.$ which means that $x_{i}(S) \geq$ $\bar{v}(\{i\})$.

This this should hold for every $i \in S$, it follows that $x(S)$ is an imputation for $\left.\vec{v}(p)\right|_{s}$.

(b) Let $x_{i}(S) \geq \tilde{v}(p,\{i\})$ for every $S$ and every $i \in S$.

Let $\Gamma^{*}$ be an arbitrary subgarme. Let $S$ be the coalition of players who have not 'deviated' before reaching this subgame and let $i \in S$.

Suppose that $i$ is a player in $S$ and all players in $S \backslash i$ play "always deviate" in $\Gamma^{*}$. If $i$ chooses "always deviate" in $\Gamma^{*}$, he gets $x_{i}(S)$, whereas other pure strategies in $\Gamma^{*}$ give him at most $\max \left\{x_{i}(S), \vec{v}(p,\{i\})\right.$ which is equal to $x_{i}(S)$. Since this holds for every $i \in S$, 'everybody always deviate' constitutes a Nash equilibrium in $\Gamma^{*}$. This holds for every subgame $\Gamma^{*}$, 'everybody always deviate' is a subgame perfect equilibrium.

As a consequence of the two theorems above, we obtain the following result which shows that there is a relationship between the imputation set of $e(p)$ (imputation sets of the subgames of $e(p)$ ) and the question whether "everybody always deviate" can be a Nash equilibrium (subgame perfect equilibrium) in the local deviation game.

Theorem 9.6.3 Let $\langle\Gamma, c\rangle$ be a game with costly coordination and $p$ a MSP. Then, the following holds.

(1) There is an allocation mechanism $x$ such that 'everybody always deviate" is a Nash equilibrium in $\Gamma(p, x)$ if and only if the imputation set of $e(p)$ is 
non-empty.

(2) There is an allocation mechanism $x$ such that 'everybody always deviate' is a subgame perfect equilibrium in $\Gamma(p, x)$ if and only if every subgame of $e(p)$ has a non-empty iroputation set.

Proof. We only give a proof for (1) since the proof for (2) procedes in an anallogous way.

(a) Let $x$ be an allocation mechanism such that 'everybody always deviate' is a Nash equilibrium in $\Gamma(p, x)$. By Theorem 9.6.1, it follows that $x_{i}(N) \geq \bar{v}(p,\{i\})$ for all $i \in N$ which implies that $\bar{v}(p, N)=\sum_{i \in N} x_{i}(N) \geq$ $\sum_{i \in N} \bar{v}(p,\{i\})$. Since $e(p, N)=\bar{v}(p, N)-\sum_{i \in N} v_{i}(p)$ it follows that

$$
e(p, N) \geq \sum_{i \in N}\left(\bar{v}(p,\{i\})-v_{i}(p)\right)=\sum_{i \in N} e(p,\{i\}) .
$$

Hence, the TU-game $e(p)$ has a non-empty imputation set.

(b) Let $e(p)$ have a non-empty imputation set, which means that $e(p, N) \geq \sum_{i \in N} e(p,\{i\})$. Then, in the same way as above, it follows that $\bar{v}(p, N) \geq \sum_{i \in N} \bar{v}(p,\{i\})$. Hence, we can find an allocation mechanism $x$ such that $x_{i}(N) \geq \bar{v}(p,\{i\})$ for every $i \in N$. With Theorem 9.6.1, it follows that 'everybody always deviate' is a Nash equilibrium in $\Gamma(p, x)$.

In the following theorem, we show that there is a strong connection between the balancedness of the TU-game $e(p)$ and the question whether "everybody always deviate' can be a NE-equilibrium in the corresponding local deviation game.

Theorem 9.6.4 Let $\langle\Gamma, c\rangle$ be a game with costly coordination and $p$ a MSP. Then, there is an allocation mechanism $x$ for $p$ such that 'everybody always deviate' is a NE-equilibrium in $\Gamma(x, p)$ if and only if the game $e(p)$ is balanced.

Proof. (a) Let $x$ be an allocation mechanism such that $\sigma:=$ "everybody always deviate' is a NE-equilibrium in $\Gamma(p, x)$.

Let $S$ be an arbitrary coalition of players and let $\tau_{S}$ be a pure strategy profile for $S$ in which all members of $S$ do 'not deviate' in period 1 but 'deviate' in period 2.

In the original BSP $\sigma$, the coalition $S$ gets $\sum_{i \in S} x_{i}(N)$ whereas $S$ gets $\sum_{i \in S} x_{i}(S)=\bar{v}(p, S)$ in the BSP $\sigma \backslash \tau_{S}$. Since 'everybody always deviate' is a NE-equilibrium, it must hold that $\sum_{i \in S} x_{i}(N) \geq \bar{v}(p, S)$.

Now, define the vector $\bar{x} \in \mathbb{R}^{N}$ by $\bar{x}_{i}:=x_{i}(N)-v_{i}(p)$ for all $i \in N$. Then, it 
follows for every coalition $S$ that

$$
\begin{aligned}
\sum_{i \in S} \bar{x}_{i} & =\sum_{i \in S}\left(x_{i}(N)-v_{i}(p)\right) \\
& =\sum_{i \in S} x_{i}(N)-\sum_{i \in S} v_{i}(p) \\
& \geq \bar{v}(p, S)-\sum_{i \in S} v_{i}(p)=e(p, S) .
\end{aligned}
$$

Furthermore,

$$
\sum_{i \in N} \tilde{x}_{i}=\sum_{i \in N} x_{i}(N)-\sum_{i \in N} v_{i}(p)=\bar{v}(p, N)-\sum_{i \in N} v_{i}(p)=e(p, N) .
$$

Hence, we may conclude that $\bar{x} \in$ Core $(e(p))$ which implies that the game $e(p)$ is balanced.

(b) Let the game $e(p)$ be balanced, which means that we can find a core vector $\bar{x}$ for $e(p)$. We define the allocation mechanism $x$ in the following way. For every $i \in N$, let $x_{i}(N):=\bar{x}_{i}+v_{i}(p)$. In the same way as in (a), but now using the opposite direction, it can be shown that $x(N)$ is an efficient allocation of $\bar{v}(N)$ and

$$
\sum_{i \in S} x_{i}(N) \geq \bar{v}(p, S)
$$

for all coalitions $S$.

For every coalition $S \neq N$ we define $x(S)$ by

$$
x_{i}(S):=x_{i}(N)-\frac{1}{|S|}\left(\sum_{i \in S} x_{i}(N)-\bar{v}(p, S)\right)
$$

for all $i \in S$. Then, $x(S)$ is an efficient allocation of $\bar{v}(p, S)$ among the members of $S$ and $x_{i}(S) \leq x_{i}(N)$ for all $i \in S$.

Let $\sigma:=$ 'everybody always deviate', $S$ an arbitrary coalition and $\tau_{S}$ an arbitrary pure strategy profile for $S$. We show that $\sum_{i \in S} u_{i}\left(\sigma \backslash \tau_{S}\right) \leq \sum_{i \in \mathcal{S}} u_{i}(\sigma)$ in the local deviation game $\Gamma(p, x)$.

For every period $n$, let $T_{n}$ be the coalition of players which 'deviate' in period $n$ on the unique equilibrium path of $\sigma \backslash \tau_{S}$. Furthermore, let $T_{0} \subset S$ be the coalition of players who 'never deviate' on the equilibrium path of $\sigma \backslash \tau_{s}$. Then, it follows that

$$
\begin{aligned}
\sum_{i \in S} u_{i}\left(\sigma \backslash \tau_{S}\right) & =\sum_{n} \sum_{i \in S \cap T_{n}} x_{i}\left(T_{n}\right)+\sum_{i \in T_{0}} v_{i}(p) \\
& \leq \sum_{n} \sum_{i \in S \cap T_{n}} x_{i}(N)+\sum_{i \in T_{0}} x_{i}(N) \\
& =\sum_{i \in S} x_{i}(N) .
\end{aligned}
$$


Here, the inequality follows from the fact that $x_{i}(S) \leq x_{i}(N)$ for every coalition $S$ and $v_{i}(p) \leq v(p,\{i\}) \leq x_{i}(N)$.

Since this holds for every coalition $S$ and every pure strategy profile $\tau_{S}$ for $S$, it follows that "everybody always deviate" is a $\mathrm{NE}$-equilibrium in $\Gamma(p, x)$.

\subsection{Minimal excess solutions}

In section 3, we have seen that some games with costly coordination have no negative excess equilibria which means that it is impossible to find MSP's in those games which are completely stable against collective deviation of every coalition. In view of this existence problem, we want to develop a nonerrpty solution for games with costly coordination which selects for every game those MSP's which are maximally stable against collective deviations by coalitions. To this purpose, the excess vector of a MSP can be used as a local parameter which meesures this stability. If the entries in the excess vector are high, the stability is low and vice versa. A MSP could be called maximally stable if the corresponding excess vector is 'minimal'. However, the excess vector is a multi-dimensional parameter and therefore it is not clear how we should define 'minimal'.

One way to do this is by assuming the existence of a relation on the set of possible excess vectors and selecting those MSP's which have a minimal excess vector with respect to this relation. Solutions of this kind are called minimal excess solutions and their existence can be guaranteed under mild conditions on the relation.

\section{Definition of minimal excess solutions}

A relation $\preceq$ on the space $\mathbb{R}^{n}$ is called standard if it is

1. reflexive

2. transitive

3. imonotonic and

4. continuous.

By monotonic we mean that $e \preceq f$ whenever $e \leq f$.

We call $\preceq$ continuous if, whenever $e^{k}$ converges to $e, f^{k}$ converges $f$ and $e^{k} \preceq f^{k}$ for every $k$, it holds that $e \preceq f$.

A system of relations which assigns to every finite set $N$ a standard relation $\preceq$ on the space $\mathbb{R}^{2^{N}}$ is called a standard system of relations. For salse of convenience, we denote such a system also by $\preceq$. 
Let $\preceq$ be a standard system of relations. The solution $\varphi$ which assigns to every game with costly coordination $\langle\Gamma, c\rangle$ the set

$$
\varphi(\langle\Gamma, c\rangle):=\{p \mid e(p) \preceq e(q) \text { for all MSP's } q\}
$$

is called the minimal excess solution (ME-solution) induced by $\preceq$. Members of $\varphi(\Gamma)$ are called $M E$-equilibria.

In the remainder of this chapter we will just state ME-solution and MEequilibria without referring to the specific system of relations $\preceq$ if it is clear which system of relations we use.

\section{Existence of ME-equilibria}

It can be shown that for every standard system of relations, the ME-solution is non-empty.

Theorem 9.7.1 Let $\preceq$ be a standard system of relations and $\langle\Gamma, c\rangle$ a game with costly coordination. Then, there is at least one ME-equilibrium in $\langle\Gamma, c\rangle$.

Proof. The function $e$ which assigns to every MSP $p$ the excess vector $e(p)$ is continuous. Since the set of MSP's is compact, the set $E$ of possible excess vectors is compact as well because it is the image of a compact set under the continuous function $e$. The question whether there is a ME-equilibrium reduces to the question whether $E$ has a minimal element w.r.t. $\preceq$. Consider for every $a \in E$ the set

$$
B(a):=\{b \mid a \prec b\} .
$$

Here, $a \prec b$ means that $a \preceq b$ and $b \preceq a$. In order to show that $B(a)$ is open, we have to show that for every $b \in B(a)$ there is an open neighborhood $N(b)$ which is contained in $B(a)$. Suppose that there is a $b \in B(a)$ for which such a neighborhood does not exist. Then, there is a sequence $b^{k}$ converging to $b$ with $b^{k} \preceq a$. Since $\preceq$ is continuous, this would imply that $b \preceq a$ which is a contradiction. Hence, we may conclude that $B(a)$ is open.

Suppose that $E$ would not have a minimal element, which means that for every $b \in E$ there is an $a \in E$ with $a \prec b$. This implies that $\cup_{a \in E} B(a)$ is an open cover of $E$. Since $E$ is compact, there is a finite subcover $\cup_{a \in A} B(a)$ of $E$ where $A$ is a finite subset of $E$. Since $A$ is finite, there is a minimal element $\vec{a}$ of $A$. By definition of the sets $B(a), \bar{a}$ is minimal element in $E$, which is a contradiction to our assumption. This leads to the conclusion that $E$ has a minimal element which guarantees the existence of a ME-equilibrium. 


\subsection{Examples of minimal excess solutions}

In this section we discuss two types of ME-solutions, namely the lexicographical ME-solution and the class of linear ME-solutions, and illustrate the solutions by several examples.

\section{The lexicographical $M E$-solution}

One possible way to construct a ME-solution is by choosing the relation $\preceq$ on $\mathbb{R}^{2^{N}}$ equal to the lexicographical ordering. The ME-solution obtained in this way is called the lexicographical ME-solution.

The nature of the lexicographical ME-solution is somewhat similar to that of the nucluolus, since both concepts search for points where, roughly speaking, the maximal excess is as small as possible. However, in contrast to the nucleolus, the lexicographical ME-solution does not, in general, lead to a unique point.

Example 9.3. Consider a game with costly coordination $\langle\Gamma, c\rangle$ in which $\Gamma$ is equal to

$$
\Gamma=\left[\begin{array}{ll}
1,1 & 3,0 \\
0,3 & 2,2
\end{array}\right] .
$$

For every MSP $p=(\lambda T+(1-\lambda) B, \mu L+(1-\mu) R)$ the excess vector is equal to

$$
\begin{array}{|r|l|}
\hline 1 & 1-\lambda \\
\hline 2 & 1-\mu \\
\hline 12 & \lambda+\mu-c(12) \\
\hline
\end{array}
$$

Therefore, $e(p, 1)+e(p, 2)+e(p, 12)=2-c(12)$ for every MSP $p$. As a consequence,

$$
e^{*}:=\begin{array}{|c|c|}
\hline 1 & \frac{2-c(12)}{3} \\
\hline 2 & \frac{2-c(12)}{3} \\
\hline 12 & \frac{2-c(2)}{3} \\
\hline
\end{array} \quad<e(p) \text { for all } p .
$$

Case 1: $\quad c(12) \leq 2$.

In this case, $p^{*}:=\left(\frac{1+c(12)}{3} T+\frac{2-c(12)}{3} B_{3} \frac{1+c(12)}{3} L+\frac{2-c(12)}{3} R\right)$ is the unique MSP with excess vector $e^{*}$. Therefore, it follows immediately that $p^{*}$ is the unique MEmequilibrium.

Case 2: $\quad c(12)>2$.

In this case, $e(p, 12)<0$ and $e(p, 1), e(p, 2) \geq 0$ for all $p$. Since $(T, L)$ is the 
unique MSP where $e(p, 1)=e(p, 2)=0$ it follows that $(T, L)$ is the unique ME-equilibrium.

Example 9.4. Let $\langle\Gamma, c\rangle$ be a three person game with costly coordination with $\Gamma$ equal to

$$
\left[\begin{array}{ll}
1,1,0 & 3,0,0 \\
0,3,0 & 2,2,0
\end{array}\right] \quad\left[\begin{array}{ll}
0,0,0 & 0,0,0 \\
0,0,0 & 0,0,0
\end{array}\right]
$$

We have seen that the excess vector for a MSP

$$
p=(\lambda T+(1-\lambda) B, \mu L+(1-\mu) R, \nu W+(1-\nu) E)
$$

is given by

\begin{tabular}{|r|l|}
\hline 1 & $\nu(1-\lambda)$ \\
\hline 2 & $\nu(1-\mu)$ \\
\hline 3 & 0 \\
\hline 12 & $\nu(\lambda+\mu)-c(12)$ \\
\hline 13 & $\nu(1-\lambda)+(1-\nu)(3-2 \mu)-c(13)$ \\
\hline 23 & $\nu(1-\mu)+(1-\nu)(3-2 \lambda)-c(23)$ \\
\hline 123 & $\nu(\lambda+\mu)+(1-\nu) 4-c(123)$ \\
\hline
\end{tabular}

(a) Let $c(S)=0$ for all coalitions $S$. Then, the sum of all excesses at the MSP $p$ is equal to

$$
\nu 4+(1-\nu)(10-2 \lambda-2 \mu)
$$

which is greater or equal to 4 for every MSP. Since $e(p, 3)=0$ for every $p$, it follows that for every MSP either the maximal excess is strictly greater than $\frac{2}{3}$ or all excesses except $e(p, 3)$ are equal to $\frac{2}{3}$. Let $e^{*}$ be the vector in $\mathbb{R}^{2^{N}}$ given by $e^{*}(3)=0$ and $e^{*}(S)=\frac{2}{3}$ for all other coalitions. In view of the insight above, it follows that $e^{*} \preceq e(p)$ for every MSP $p$.

Consider the MSP

$$
p^{*}=\left(\frac{1}{3} T+\frac{2}{3} B, \frac{1}{3} L+\frac{2}{3} R, W\right) .
$$

Since $e\left(p^{*}\right)=e^{*}$ and $p^{*}$ is the unique MSP with this excess vector, it follows that $p^{*}$ is the unique ME-equilibrium in this game with costly coordination.

(b) Now, let $c$ be given by $c(12)=1, c(13)=c(23)=4$ and $c(123)=5$.

It can be seen easily that in this case, $e(p, 13)<e(p, 1), e(p, 23)<e(p, 2)$ and $e(p, 123)<e(p, 12)$ for every MSP $p$. Therefore, every ME-equilibrium must have the property that the excess vector restricted to the coalitions $\{1\},\{2\},\{3\}$ and $\{1,2\}$ is lexicographically minimal. Obviously, this restricted excess vector is only lexicographically minimal if $\nu=0$. In order 
to make the excess vector restricted to the other coalitions lexicographically minimal, we must choose $\lambda=\mu=1$. Therefore, $(T, L, E)$ is the unique ME-equilibrium in this game with costly coordination.

\section{Linear ME-solutions}

Another way to generate ME-solutions is to choose $\preceq$ equal to a linear relation. By a linear relation we mean a relation $\preceq$ on $\mathbb{R}^{2^{N}}$ for which there is a non-negative vector $\alpha=(\alpha(S))_{S \in 2^{N}}$ with $\alpha(\varnothing)=0$ such that

$$
e \leq f \text { if and only if }\langle\alpha, e\rangle \leq\langle\alpha, f\rangle \text {. }
$$

Here, $\langle\alpha, e\rangle$ denotes the inner product of the vectors $\alpha$ and $e$. We say that $\alpha$ is a coefficient vector for $\measuredangle$. The ME-solutions which are generated by such linear relations are called linear ME-solutions.

If we choose, for example, $\alpha(\{i\})>0$ for all $i$ and $\alpha(S)=0$ if $|S| \geq 2$ then the MEmequilibria are exactly the Nash equilibria. This follows from the fact that in this case

$$
\langle\alpha, e(p)\rangle=\sum_{i} \alpha(\{i\}) e(p,\{i\})
$$

which is always greater or equal than zero and is equal to zero exactly at the Nash equilibria.

Lemma 9.8.1 The linear ME-equilibria of a game with costly coordination $\langle\Gamma, c\rangle$ do not depend on $c$.

Proof. For every MSP $p_{n}$ let $e^{0}(p, S)$ be given by

$$
e^{0}(p, S)=\max _{m_{S}} \sum_{i \in S} v_{i}\left(p \backslash m_{S}\right)-\sum_{i \in S} v_{i}(p)
$$

Then, it follows that

$$
e(p, S)=e^{0}(p, S)-c(S)
$$

for every MSP $p$ and every coalition $S$. Vectorwise, we yield the equation

$$
e(p)=e^{0}(p)-c
$$

where $e^{0}(p):=\left(e^{0}(p, S)\right)_{S \in 2^{N}}$ does not depend on $c$. Then,

$$
\langle\alpha, e(p)\rangle=\left\langle\alpha, e^{0}(p)\right\rangle-\langle\alpha, c\rangle
$$


So, the MSP's at which $\langle\alpha, e(p)\rangle$ is minimal are exactly the MSP's at which $\left\langle\alpha, e^{0}(p)\right\rangle$ is minimal. Since the latter function does not depend on $c$, it follows that the linear ME-equilibria w.r.t. $\alpha$ are independent of $c$.

However, we have seen in Example 9.3 that the lexicographic ME-equilibria of a game with costly coordination do depend on the choice of the cost function c.

Example 9.5. Consider a game with costly coordination $\langle\Gamma, c\rangle$ where

$$
\Gamma=\left[\begin{array}{ll}
1,1 & 3,0 \\
0,3 & 2,2
\end{array}\right]
$$

Since for every linear relation, the ME-equilibria do not depend on $c$, we assume that $c(S)=0$ for every coalition $S$. Let $\preceq$ be the linear relation induced by $\alpha=(\alpha(1), \alpha(2), \alpha(12))$.

For a MSP $p=(\lambda T+(1-\lambda) B, \mu L+(1-\mu) R)$, the excess vector is equal to

$$
e(p)=\begin{array}{|r|r|}
\hline 1 & 1-\lambda \\
\hline 2 & 1-\mu \\
\hline 12 & \lambda+\mu \\
\hline
\end{array}
$$

Therefore,

$$
\langle\alpha, e(p)\rangle=\alpha(1)+\alpha(2)+\lambda(\alpha(12)-\alpha(1))+\mu(\alpha(12)-\alpha(2)))
$$

The set of ME-equilibria, as a function of the coefficient vector $\alpha$, is depicted in the following diagram.

\begin{tabular}{|l|l|l|l|}
\hline & $\alpha(12)<\alpha(2)$ & $\alpha(12)=\alpha(2)$ & $\alpha(12)>\alpha(2)$ \\
\hline$\alpha(12)<\alpha(1)$ & $\{(T, L)\}$ & $\{T\} \times \Delta\left(M_{7}\right)$ & $\{(T, R)\}$ \\
\hline$\alpha(12)=\alpha(1)$ & $\Delta\left(M_{1}\right) \times\{L\}$ & $\Delta\left(M_{1}\right) \times \Delta\left(M_{2}\right)$ & $\Delta\left(M_{1}\right) \times\{R\}$ \\
\hline$\alpha(12)>\alpha(1)$ & $\{(B, L)\}$ & $\{B\} \times \Delta\left(M_{2}\right)$ & $\{(B, R)\}$ \\
\hline
\end{tabular}

Here, $\Delta\left(M_{i}\right)$ denotes the set of all mixed strategies for player $i$.

Example 9.6. Let $\langle\Gamma, c\rangle$ be a three person game with costly coordination where $\Gamma$ is given by

$$
\left[\begin{array}{ll}
1,1,0 & 3,0,0 \\
0,3,0 & 2,2,0
\end{array}\right] \quad\left[\begin{array}{cc}
0,0,0 & 0,0,0 \\
0,0,0 & 0,0,0
\end{array}\right]
$$

Again, we assume that $c(S)=0$ for every coalition $S$. For a MSP

$$
p=(\lambda T+(1-\lambda) R, \mu L+(1-\mu) R, \nu W+(1-\nu) E)
$$


the excess vector is given by

\begin{tabular}{|r|l|}
\hline 1 & $\nu(1-\lambda)$ \\
\hline 2 & $\nu(1-\mu)$ \\
\hline 3 & 0 \\
\hline 12 & $\nu(\lambda+\mu)$ \\
\hline 13 & $\nu(1-\lambda)+(1-\nu)(3-2 \mu)$ \\
\hline 23 & $\nu(1-\mu)+(1-\nu)(3-2 \lambda)$ \\
\hline 123 & $\nu(\lambda+\mu)+(1-\nu) 4$ \\
\hline
\end{tabular}

(a) Let $\preceq$ be the linear relation induced by the coefficient vector $\alpha$ with $\alpha(S)=1$ for every coalition $S$. Then

$$
\langle\alpha, e(p)\rangle=\nu 4+(1-\nu)(10-2 \lambda-2 \mu),
$$

which is minimal exactly when $\nu=1$. Therefore, the ME-equilibria are exactly the MSP's where player 3 plays $W$.

(b) Now, choose $\alpha(S)=1$ if $|S|=1, \alpha(S)=2$ if $|S|=2$ and $\alpha(123)=3$. Then

$$
\langle\alpha, e(p)\rangle=\nu(6+2 \lambda+2 \mu)+(1-\nu)(24-4 \lambda-4 \mu)
$$

which is minimal if and only if $\nu=1, \lambda=0$ and $\mu=0$. Hence, $(B, R, W)$ is the unique ME-equilibrium in this case.

\subsection{Characterization of ME-equilibria by lo- cal deviation games}

Let $\preceq$ be a standard relation on $\mathbb{R}^{2^{N}}$. Since the cost functions $c$ are also members of the space $\mathbb{R}^{2^{N}}$, the relation $\preceq$ can also be applied on the set of possible cost functions. An interesting problem is to find the minimal cost functions w.r.t. $\preceq$ which guarantee the existence of NE-equilibria. We show that this problem is equivalent to the problem of finding ME-equilibria in a game with costly coordination where the coordination costs are zero and the relation is strictly monotonic. Combining this result with the characterizations of NE-equilibria in section 5 leads to several characterizations of MErequilibria by means of the local deviation game. Before we come to these results, we need some more definitions.

Let $\Gamma$ be a normal form game and $\preceq$ a standard system of relations. By $C(\Gamma)$ we denote the set of cost vectors $c$ for which the garne with costly 
coordination $\langle\Gamma, c\rangle$ contains a NE-equilibrium. Furthermore, we define the set

$$
C^{* *}(\Gamma):=\{c \in C(\Gamma) \mid c \preceq \bar{c} \text { for all } \bar{c} \in C(\Gamma)\}
$$

of minimal elements in $C(\Gamma)$.

We say that the relation $\preceq$ is strictly monotonic if $e \leq f$ and $e(S)<f(S)$ for some coalition $S$ implies that $e \prec f$.

Strictly monotonic relations are for, example, the lexicographic relation and linear relations where all the coefficients $\alpha(S)$ are strictly positive. It turns out that for such relations there is a strong connection between $C^{*}(\Gamma)$ and ME-equilibria, as is shown in the following theorem.

Theorem 9.9.1 Let $\preceq$ be a standard system of relations which is strictly monotonic and $\langle\Gamma, c\rangle$ a game in which all coordination costs are zero. Then, $C^{*}(\Gamma)$ is the set of excess vectors $e(p)$ generated by $M E$-equilibria $p$ in $\langle\Gamma, c\rangle$.

Proof. Since we vary the cost vector in this proof, we denote the excess vector of $p$ in the game with costly coordination $\langle\Gamma, \vec{c}\rangle$ by $e(p, \bar{c})$ instead of $e(p)$.

The set $C(\Gamma)$ consists, by definition, exactly of those cost vectors $\bar{c}$ for which there is a MSP $p$ such that $e(p, \bar{c}) \leq 0$ in the game with costly coordination $\langle\Gamma, \bar{c}\rangle$. Since $e(p, \bar{c})=e(p, c)-\bar{c}$, it follows that $C(\Gamma)$ is the set of cost vectors $\bar{c}$ for which there is a MSP $p$ with $\bar{c} \geq e(p, c)$.

By strict monotonicity of $\preceq$, all minimal elements of $C(\Gamma)$ are member of the set

$$
\bar{C}:=\{e(p, c) \mid p \text { MSP }\}
$$

Therefore,

$$
\begin{aligned}
C^{*}(\Gamma) & =\{\bar{c} \in \bar{C} \mid \vec{c} \preceq d \text { for all } d \in \bar{C}\} \\
& =\{e(p, c) \mid e(p, c) \preceq e(\bar{p}, c) \text { for all MSP's } \bar{p}\} \\
& =\{e(p, c) \mid p \text { ME-equilibrium in }\langle\Gamma, c\rangle\} .
\end{aligned}
$$

It follows that $p$ is a ME-equilibrium in the game $\langle\Gamma, c\rangle$ with coordination costs zero if and only if $e(p) \in C^{*}(\Gamma)$, which is equivalent to the statement. in the following corrolary.

Corollary 9.9.2 Let $\langle\Gamma, c\rangle$ be a game with coordination costs zero and $\preceq$ a standard and strictly monotonic relation on $\mathbb{R}^{2^{N}}$. Then, $p$ is a $M E-$ equilibrium in $\langle\Gamma, c\rangle$ if and only if $e(p)$ is a minimal cost vector for which $\langle\Gamma, e(p)\rangle$ has a NE-equilibrium. 
If we combine this characterization with the results in section 5 , we obtain the following characterizations of ME-equilibria in zero costs games by means of the local deviation game.

Theorem 9.9.3 Let $\langle\Gamma, c\rangle$ be a game with costly coordination with zero coordination costs and $\preceq$ a standard and strictly monotonic relation on $\mathbb{R}^{2^{N}}$. Then,

(1) $p$ is a $M E$-equilibrium in $\langle\Gamma, c\rangle$ if and only if $e(p)$ is a minimal cost vector for which 'everybody never deviate' is a NE-equilibrium in a local deviation game $\Gamma(\bar{p}, x)$.

(2) $p$ is a ME-equilibrium in $(\Gamma, c\rangle$ if and only if $e(p)$ is a minimal cost vector for which 'everybody never deviate' is a strong Nash equilibrium in a local deviation game $\Gamma(\tilde{p}, x)$ in which $x$ is one sided.

This characterization holds in particular for the lexicographical ordering and linear relations in which all coefficients are strictly positive.

The same characterizations hold if we replace 'NE-equilibrium' in (1) by 'subgame perfect NE-equilibrium' or replace 'everybody never deviate is a strong Nash equilibrium' in (2) by 'never deviate is a dominant strategy for each player".

If the relation $\preceq$ is in addition translation invariant, which means that $e \preceq f$ implies $e+c \preceq f+c$ for every $c \in \mathbb{R}^{2^{N}}$, then we can generalize the above characterizations for general games with costly coordination, in which the costs are not necessarily zero.

Theorem 9.9.4 Let $\langle\Gamma, c\rangle$ be a game with costly coordination and $\preceq$ a standard, strictly monotonic and translation invariant relation on $\mathbb{R}^{2^{N}}$. Then, (1) $p$ is a ME-equilibrium in $\langle\Gamma, c\rangle$ if and only if $c+e(p)$ is a minimal cost vector for which 'everybody never deviate' is a. NE-equilibrium in a local deviation game $\Gamma(\bar{p}, x)$.

(2) $p$ is a ME-equilibrium in $\langle\Gamma, c\rangle$ if and only if $c+e(p)$ is a minimal cost vector for which 'everybody never deviate' is a strong Nash equilibrium in local deviation game $\Gamma(\bar{p}, x)$ in which $x$ is one sided.

Proof. By translation invariance of $\preceq$, it follows that the ME-equilibria of $\langle\Gamma, c\rangle$ coincide with the ME-equilibria of the game with costly coordination $\langle\Gamma, 0\rangle$ where the coordination costs are zero. Obviously, $e(p, 0)=c+e(p, c)$ for every MSP $p$, where $e(p, 0)$ is the excess of $p$ in the game $\langle\Gamma, 0\rangle$. Using Theorem 9.9.1, it follows that $C^{*}(\Gamma)$ consists exactly of the vectors $c+e(p, c)$ where $p$ is a ME-equilibrium in $\langle\Gamma, c\rangle$. This implies the required results. 
Since every linear relation is translation invariant, the above characterizations hold in particular for linear relations in which all coefficients are strictly positive. 


\section{Index}

\section{A}

action, 19

agent, 19

agent normal form, 143

answer, 42

assessment, 20

assessment solution, 169

B

b-labelling, 22

balanced game, 192

base game, 69

Bayesian consistent assessment, 20, 44

behavior strategy , 20

belief, 20

C

chance node, 19

closed family of games, 161

completely mixed strategy, 161

consistency in signaling games, 44

consistent assessment, 20

consistent solution, 162

conversely consistent solution, 162

cooperative excess game, 190

cooperative game, 180

\section{D}

dependent player splitting, 153

detectable deviation, 124

dominant strategy, 186

$\mathbf{E}$ edge, 19

equivalent extensive form games, 139 excess, 181

expected payoff, 20

extended game, 69,120

extensive form game, 19

extensive form structure, 19

F

full commitment case, 106

G

game with costly coordination, 181

global sequential rationality, 20

greater action, 119

greater information mechanism, 123

H

history, 118

I

imputation, 190

independent player splitting, 138

indistinguishable actions, 119

infinite signaling game, 42

information buyer, 69

information mechanism, 117

information mechanism inspection, 125

information seller, 69

information set, 19

information value, 74

invariant solution, 149 


\section{K}

Kohlberg-Mertens stable set, 148

\section{L}

limit consistent solution, 166

local deviation game, 185

local sequential rationality, 20

logarithmic cone, 34

lower equilibrium, 118

\section{M}

Mertens stable set, 149

message, 42

minimal excess solution, 195

mixed strategy, 138

more informative action, 119

\section{$\mathrm{N}$}

Nash equilibrium, 142

negative excess equilibrium, 182 negative excess solution, 182

no commitment case, 101

node, 19

normal form, 138

normal form game, 161

O

optimal sequential equilibrium, 73

$\mathbf{P}$

path, 19

payoff, 19

perfect equilibrium, 143, 168

perfect equivalence, 152

perfect recall, 19

personalized Nash equilibrium, 163

player splitting, 138

player splitting property, 139

pointwise convergence, 48

proper equilibrium, 146

pseudo-behavior strategy , 21

public information transaction, 86

pure strategy, 138
$\mathbf{R}$

reduced assessment, 75

reduced behavior strategy, 75

reduced belief system, 75

reduced game, 161,169

reduced local strategy, 75

reduced sequential equilibrium, 76

repeated game, 117

reservation value, 78

root, 19

S

semi-algebraic set, 35

separable metric space, 42

sequential equilibrium, 21,44

sequential rationality, 20,43

signal, 117

signaling game, 42

simple behavior strategy, 121

simple lower equilibrium, 121

simple sequence of assessments, 29

single appearance game, 69,157

single appearance structure, 157

solution, 162

stable set, $148-149$

standard case, 110

standard negotiation phase, 107

strong Nash equilibrium, 182

strongly consistent assessment, 52 strongly consistent solution, 162

subgame perfect equilibrium, 185

$\mathrm{T}$

terminal node, 19

TU-game, 180

type, 42

U

undetectable deviation, 124

W

weak convergence, 51 
weak convergence of assessments, 46

weak invariance under player splittings, 153

weak player splitting property, 140 weakly perfect equilibrium, 170

weight matrix, 119 


\section{Bibliography}

Admati and Pfleiderer (1986). A monopolistic market for information. Journal of Economic Theory 39, 400-438.

Allen (1986). The demand for (differential) information. Review of Economic Studies 53, 31I-323.

Aumann, R. J. (1959). Acceptable points in general coopenative n-person games. in "Contributions to the Theory of Games IV" (H.W. Kuhn and R.D. Luce, Eds.), pp. 287-324. Princeton: Princeton Univ. Press.

Aumann, R.J. (1964). Mixed and behavior strategies in infinite exterssive games. In: Dresher $\mathrm{M}$ et al. (eds) Advances in Game Theory. Princeton University Press, Princeton, NJ, Ann. Math. Studies 53, 627-650.

Battigalli, P. (1996). Strategic independence and perfect Bayesian equilibria. Journal of Economic Theory 70, 201-234.

Bernbeim, B.D., Peleg, B. and M.D. Whinston (1987). Coalition-proof Nash equilibria. I. Concepts. Journal of Economic Theory 42, 1-12.

Billingsley, P. (1968). Convergence of probability measures. New York: John Wiley \& Sons.

Billingsley, P. (1986). Probability and Measure. New York: John Wiley \& Sons.

Blume, L.E., and W.R. Zame (1994). The algebraic geometry of perfect and sequential equilibrium. Econometrica 62, 783-794

Cho, I.-K. and D.M. Kreps (1987). Signaling games and stable equilibria. The Quarterly Journal of Economics 102, 179-221.

Cook, W., Gerards, A.M.H., Schrijver, A. and Tardos, E. (1986). Sensitivity theorems in integer linear programming. Mathematical Programming 34, 48-61. 
Flesch, J. and Perea y Monsuwé, A. (1997). Repeated games with many information mechanisms. Working paper.

Fudenberg, D. and D. Levine (1989). An approximate Folk Theorem with imperfect private information. Mimeo.

Fudenberg, D. and E. Maskin (1986). Discounted repeated games with unobservable actions. I. One-sided moral hazard. Mimeo.

Fudenberg, D. and J. Tirole (1991). Perfect Bayesian equilibrium and sequential equilibrium. Journal of Economic Theory 53, 236-260.

Gilboa and Lehrer (1991). The value of information. Journal of Mathematical Economics 20, 443-459.

Green (1981). Value of information with sequential future markets. Econometrica $49,335-358$.

Hendon, E., Jacobsen, J. and B. Sloth (1996). The one-deviation principle for sequential rationality. Games and Economic Behavior 12, 274-282.

Kamien, M.I., Tauman, Y. and S. Zamir (1990). On the value of information in a strategic conflict. Games and Economic Behavior 2, 129-153.

Kohlberg, E. and J.-F. Mertens (1986). On the strategic stability of equilibria. Econometrica 54, 1003-1039.

Kohlberg, E., and Reny, P.J. (1991). On the rationale for perfect equilibrium (provisional title). Working paper.

Kreps, D.M., and Wilson, R. (1982). Sequential equilibria. Econometrica 50, 863-894.

Kuhn, H.W. (1953). Extensive form games and the problem of information. Annals of Mathernatics Studies 28, 193-216.

Lehrer, E. (1989). Lower equilibrium payoffs in two-player repeated games with non-observable actions. International Journal of Game Theory 18, 5789.

Lehrer, E. (1990). Nash equilibria of n-player repeated games with semistandard information. International Journal of Game Theory 19, 191-217.

Lehrer, E. (1991). Internal correlation in repeated games. International Journal of Game Theory 19, 431-456.

Liehrer, E. (1992a). Correlated equilibria in two-player repeated games with nonobservable actions. Mathematics of Operations Research 17, 175-199.

Lehrer, E. (1992b). Two-player repeated games with nonobservable actions 
and observable payoffs. Mathematics of Operations Research 17, 200-224.

Levine and Ponssard (1977). The values of information in some non-zero sum games. International Journal of Game Theory 6, 221-229.

Mailath, G.J. (1987). Incentive compatibility in signaling games with a continuum of types. Econometrica 55, 1349-1365.

Mailath, G.J. (1988). On the behavior of separating equilibria of signaling games with a finite set of types as the set of types becomes dense in an interval. Journal of Economic Theory 44, 413-424.

Manelli, A. (1996). Cheap Talk and Sequential Equilibria in Signaling Games. Forthcoming in Econometrica.

McLennan, A. (1989a). The space of conditional systems is a ball. International Journal of Game Theory 18, 125-139.

McLennan, A. (1989b). Consistent conditional systems in non-cooperative game theory. International Journal of Game Theory 18, 141-174.

Mertens, J.-F. (1987). Ordinality in non cooperative games. Core Discussion Paper 8728, CORE Louvian de la Neuve, Belgium.

Mertens, J.-F. (1989). Stable equilibria - a reformulation, Part I. Mathematics of Operations Research 14, 575-625.

Milgrom, P. and J. Roberts (1996). Coalition-proofness and comelation with arbitrary communication possibilities. Games and Economic Behavior 17, 133-128.

Milne and Shefrin (1987). Information and securities: A note on Pareto dominance and the second best. Journal of Economic Theory 43, 314-328.

Moreno, D. and J. Wooders (1996). Coalition-proof equilibrium. Games and Economic Behavior 17, 80-1.12.

Munkres, J.R. (1984). Elements of algebraic topology. Addision-Wesley Publishing Company, Reading, Massachusetts.

Myerson, R.B. (1978). Refinements of the Nash equitibrium concept. International Journal of Game Theory 7, 73-80.

Myerson, R.B. (1991). Game theory, Analysis of conflict. Harvard University Press, Cambridge, Massachusetts.

Nash, J.F. (1950). Equilibrium points in n-person games. Proceedings from the National Academy of Science, U.S.A. 36, 48-49.

Neyman, A. (1991). The positive value of information. Games and Economic 
Behavior 3, 350-355.

Norde, H., Potters, J., Reijnierse, H. and D. Vermeulen (1996). Equilibrium selection and consistency. Games and Economic Behavior 12, 219-225.

Patrone, F., Pieri, G., Tijs, S. and A. Torre (1995). On consistent solutions for strategic games. Working Paper, Department of Mathematics, Genoa, Italy.

Peleg, B. and S. Tijs (1996). The Consistency Principle for Games in Strategic Form. International Journal of Game Theory 25, 13-34.

Perea y Monsuwé, A., Jansen, M. and H. Peters (1996a). Consistency of assessments in infinite signaling games. Forthcoming in Journal of Mathematical Economics.

Perea y Monsuwé, A., Jansen, M. and H. Peters (1996b). Characterization of consistent assessments in extensive form games. Forthcoming in Games and Economic Behavior.

Perea y Monsuwé, A. and H. Peters (1996c). Limit consistent solutions in non-cooperative games. Research Memorandum RM/96/014.

Perea y Monsuwé, A., Jansen, M. and A. Vermeulen (1996d). Player splitting in extensive form games. Working paper.

Perea y Monsuwé, A. (1997a). Buying information in extensive form games. Working paper.

Perea y Monsuwé, A. (1997b). Non-cooperative games with costly coordination. Working paper.

Perea y Monsuwé, A. and J. Swinkels (1997). Buying information and the seller's commitment degree. Working paper.

Radner, R. (1986). Repeated parthership games with imperfect monitoring and no discounting. Review of Economic Studies 53, 43-58.

Ray, I. (1993). Coalition-proof correlated equilibrium: A definition. Games and Economic Behavior 17, 56-79.

Rockafellar, R.T. (1970). Convex analysis. Princeton University Press, Princeton, New Jersey.

Sakai (1985). The value of information in a simple duopoly model. Journal of Economic Theory 36, 36-54.

Selten, R. (1965). Spieltheoretische Behandlung eines Oligopolmodells mit Nachfrageträgheit. Zeitschrift für die gesamte Staatswissenschaft 121, 301324 and 667-689. 
Selten, R. (1975). Reexamination of the perfectness concept for equilibrium points in extensive games. International Journal of Game Theory 4, 25-55.

Simon, L.K. and M.B. Stinchcombe (1995). Equilibrium refinement for infinite normal- form games. Econometrica 63, 1421-4143.

Van Damme, E. (1987). Equilibria in Non-Cooperative Games. in Surveys in Game Theory and Related Topics (H. Peters and O. Vrieze, Eds.) C.W.I. Tract 39. Amsterdam, 1987.

Van Damme, E. (1991). Stability and Perfection of Nash Equilibria. Berlin: Springer Verlag. 



\section{Summary in Dutch}

In dit proefschrift worden enkele eigenschappen van niet-coöperatieve spelen onderzocht. Een niet-coöperatief spel is een wiskundig hulpmiddel dat gebruikt wordt om situaties te analyzeren waarbij individuen (of groepen) beslissingen moeten nemen zonder dat er bindende afspraken tussen de individuen (of groepen) mogelijk zijn. Deze individuen c.q. groepen worden spelers genoemd.

We behandelen in dit proefschrift drie klassen van niet-coöperatieve spelen, te weten spelen in uitgebreide vorm, spelen in normale vorm en herhaalde spelen. In een spel in uitgebreide vorm wordt het patroon, volgens welke de beslissingen moeten worden genomen, vastgelegd door een boom, bestaande uit knopen en kanten. Iedere knoop stelt een situatie woor waarin ofwel een speler een beslissing moet nemen, ofwel een toevallige gebeurtenis plaatsvindt waarop de spelers geen invloed kunnen uitoefenen. In het eerste geval worden de mogelijke beslissingen gerepresenteerd door de kanten die de knoop verlaten. Iedere kant (lees: beslissing) leidt weer tot een nieuwe knoop, enzovoort. Als een eindknoop bereikt wordt, dan betekent dit dat het spel is afgelopen. Elke speler krijgt dan een zogenaamde witbetaling, welke geinterpreteerd kan worden als de beloning voor deze speler als het spel de betreffende eindknoop bereikt.

In veel gevallen is een speler echter niet geheel op de hoogte van de beslissingen die door anderen in het verleden genomen zijn of de uitkomst van een toevallige gebeurtenis. Technisch gesproken betekent dit dat een speler niet precies weet in welke knoop hij zich bevindt. Dit gebrek aan informatie wordt als volgt gemodelleerd. De knopen waartussen een speler op voorhand geen onderscheid kan maken worden samengevoegd tot een zogenaamde informatieverzameling. Als het spel een knoop in deze informatie-verzameling bereikt, dan weet de betreffende speler slechts dat hij zich in een der knopen van de informatie-verzameling bevindt. Hij weet echter niet in welke der knopen. Men veronderstelt dat de speler aan iedere knoop in de informatie-verzameling een bepaalde kans toekent. Deze kansen worden beiliefs genoemd. Beliefs kunnen worden opgevat als de persoonlijke inschatting van de speler betreffende de waarschijnlijkkheden waarmee de afzonderlijke knopen in een informatieverzameling bereikt worden.

Het gedrag van een speler wordt beschreven door een gedrags-strategie die aan elk van zijn informatie-verzamelingen een kansverdeling over de mogelijke beslissingen toekent. Een combinatie bestaande uit gedrags-strategieën en beliefs noemt men een assessment. 
Een van de belangrijkste oplossings-concepten voor spelen in uitgebreide vorm is het sequentieel evenwicht. Een assessment is een sequentieel evenwicht als het voldoet aan twee condities, te weten sequentiele rationaliteit en consistentie. De eerste conditie eist dat iedere speler op elk van zijn unformatie-verzamelingen optimaal handelt, gegeven zijn beliefs. De tweede conditie is iets ingewikkelder te beschrijven maar eist, grof gezegd, dat de beliefs op een bepaalde manier moeten voortvloeien uit de gedrags-strategieën. Om consistentie exact te omschrijven wordt in de originele definitie gebruik gemaakt van rijen en limieten van assessments.

In Hoofdstuk 2 laten we zien dat consistentie ook op een andere manier beschreven kan worden, zonder gebruik te maken van rijen en limieten. Dit resultaat wordt gebruikt om een algorithme te ontwikkelen waarmee de verzar meling der consistente assessments uitgerekend kan worden.

In Hoofdstuk 3 wordt onderzocht hoe consistentie gedefinieerd dient te worden in zogenaamde oneindige signaling games. Een signaling game is een spel in uitgebreide vorm dat gespeeld wordt door twee spelers. Aan het begin van het spel bepaalt een toevallige gebeurtenis (ook wel zet van de natuur genoemd) het type van speler 1 . Speler 1 kan bijvoorbeeld sterk of zwak zijn. Vervolgens zendt speler 1 een boodschap uit, die door speler 2 gezien wordt. Speler 1 kan bijvoorbeeld bier of quiche als ontbijt kiezen. Speler 2 weet niet welk type speler 1 bezit, maar aan de hand van de geobserveerde boodschap zal speler 2 beliefs vormen over de mogelijke types van speler 1 . Vervolgens zal speler 2 een antwoord geven, waarna het spel eindigt.

In een oneindig signaling game wordt verondersteld dat de types, boodschappen en antwoorden deel uitmaken van volledige, separabele metrische ruimtes, welke oneindig veel elementen kumnen bevatten (vandaar de term oneindig). Voor deze klasse van spelen bestond in de literatuur nog geen definitie van consistentie, omdat de oneindigheid van de ruimtes problemen veroorzaakt bij de convergentie van assessments. In Hoofdstuk 3 presenteren we een consistentie-definitie die we sterke consistentie noemen. Vervolgens tonen we aan dat iedere consistentie-definitie, die aan bepaalde minimale condities voldoet, een verfijning van sterke consistentie oplevert. Bovendien geven we een karakterizering van sterke consistentie in eenvoudige termen.

In de Hoofdstukken 4 en 5 breiden we het model van een spel in uitgebreide vorm uit tot een situatie waarin spelers informatie kunnen kopen van een monopolistische verkoper. Formeel betekent dit dat een speler bij elk van zijn informatie-verzamelingen een partitie kan kopen die de informatieverzameling in disjuncte deelverzamelingen opdeelt. De verkoper zal de speler meedelen welke deelverzameling van de partitie bereikt wordt. Voordat het eigenlijke spel begint, kiest de verkoper een prijs voor iedere partitie die door hem wordt aangeboden. Deze prijzen worden door iedere speler geobserveerd. 
Op deze manier onstaat een nieuw, groter spel in uitgebreide vorm, waarin de verkoper een van de spelers is. De centrale vraag die we stellen is: Hoeveel geld kan de verkoper verdienen met het verkopen van informatie?

We beantwoorden deze vraag door te zoeken naar de hoogste uitbetaling die de verkoper kan verkrijgen in een sequentieel evenwicht van het nieuwe spel. Deze uitbetaling noemen we de informatie-waande. Het nieuwe spel is geen eindig spel omdat de informatie-verkoper kan kiezen uit een continuum van prijs-vectoren. Daarom ligt het bestaan van een sequentieel evenwicht in dit spel niet voor de hand. We tonen echter aan dat zo'n sequentieel evenwicht altijd bestaat. Bovendien blijkt dat de uitbetaling voor de verkoper een maximum aanneemt op de verzameling der sequentiële evenwichten, hetgeen impliceert dat de informatie-waarde een goed gedefinieerd begrip is. Verder laten we zien dat de informatie-waarde stijgt indien de verkoper besluit de verzameling aangeboden partities uit te breiden. Het vervangen van een partitie door een fijnere partitie kan echter leiden tot een daling van de informatie-waarde. In ons model memen we aan dat de partitie die door een speler gekocht wordt, niet geobserveerd wordt door de andere spelers. We noemen dit private informatie-transactie. Aan de hand van twee voorbeelden laten we zien dat het publiek maken van de informatie-transactie kan leiden tot een stijging of een daling van de informatie-waarde.

Tot nu toe hebben we aangenomen dat de verkoper alle prijzen kiest voordat het eigenlijke spel begint. Er zijn echter ook andere manieren waarop de verkoper de prijzen kan kiezen. In Hoofdstuk 5 bekijken we twee andere manieren en onderzoeken de consequenties hiervan voor de informatiewaarde.

Allereerst bestuderen we de situatie waarin de verkoper de prijs van een partitie kiest op het moment dat de bijbehorende informatie-verzameling bereikt wordt. Sequentiële rationaliteit dwingt de verkoper om slechts die prijzen te kiezen die, gegeven het gedrag van de spelers, optimaal zijn als deze informatie-verzameling bereikt wordt. Voor de verkoper is er daarom geen ruimte voor commitment. Vandaar dat we deze situatie de no commitment case noemen.

De situatie zoals beschreven in Hoofdstuk 4 noemen we de standard case. Tenslotte bekijken we de situatie waarin de verkoper met de spelers kan onderhandelen over de prijzen van de partities voordat het eigenlijke spel begint. Deze onderhandelingspositie geeft de verkoper maximale commitment-power. Deze situatie noemen we dan ook de full commitment case.

Als hoofdresultaat tonen we aan dat de informatie-waarde in de no commitment case altijd lager is dan de informatie-waarde in de standard case, welke op haar beurt altijd lager is dan in de full commitment case.

In Hoofdstuk 6 maken we de overstap naar herhaalde spelen. Een herhaald 
spel is een situatie waarin een spel in normale vorm oneindig vaak gespeeld wordt. Met een spel in normale vorm bedoelen we een spel waarbij de spelers gelijktijdig een actie moeten kiezen. Het kan daarom opgevat worden als een bijzonder geval van een spel in uitgebreide vorm. In dit hoofdstuk concentreren we ons op herhaalde spelen met twee spelers waarbij de spelers na elke periode (tijdstip waarop het spel in normale vorm gespeeld wordt) een signaal krijgen betreffende de actie van de tegenstander. In het algemeen geeft zo'n signaal geen perfecte informatie over de actie die door de tegenstander gespeeld werd. Het mechanisme dat voor deze signalen zorgt noemen we een informatie-mechanisme. We breiden dit model uit tot een situatie waarbij beide spelers een informatie-mechanisme kunnen kopen voordat het herhaalde spel begint. Voor deze situatie geven we een karakterizering van de uitbetalingen welke gegenereerd worden door zogenaamde lower equilibria. Grof gesteld kan men zeggen dat lower equilibria een bepaald type Nash evenwichten voor herhaalde spelen vormen. Belangrijik is dat de karakterizering alleen gebruik maakt van het spel in normale vorm.

In Hoofdstuk 7 keren we terug naar spelen in uitgebreide vorm. We bestuderen de situatie waarin de informatie-verzamelingen van een speler verdeeld worden over zogenaamde agenten. Op deze manier wordt een nieuw spel verkregen waarin de agenten dezelfde uitbetalingen ontvangen als de speler waartoe ze oorspronkelijk behoorden. Zo'n opsplitsing van informatie-verzamelingen noemt men een player splitting. Worden de informatie-verzamelingen op een dusdanige manier verdeeld dat op elk pad in de boom hoogstens één agent van elke speler voorkomt, dan noemt men de player splitting anafhankelijk. We tonen aan dat de Nash evenwichten, (normale vorm) perfecte evenwichten en stabiele verzamelingen van een spel in uitgebreide vorm niet veranderen door een onafhankelijke player splitting. Een voorbeeld toont aan dat de propere evenwichten wel kunnen veranderen in zo'n geval. Bovendien laten we zien dat de klasse der onafhankelijke player splittings de grootste klasse van player splittings is waarbij de Nash evenwichten, perfecte evenwichten en stabiele evenwichten nooit veranderen.

Hoofdstuk 8 spitst zich toe op consistentie van niet-coöperatieve oplossingen. Om uit te leggen wat consistentie inhoudt, is het belangrijk om te weten wat een gereduceerd spel is. Neem een niet-coöperatief spel, kies een coalitie van spelers en selecteer voor iedere speler in deze coalitie een gemengde strategie (kansverdeling over zuivere strategieën). Het "kleinere" spel dat men werkrijgt door de spelers in deze coalitie vast te pinnen aan hun uitgekozen gemengde strategie noemt men een gereduceerd spel. Dit gereduceerde spel wordt gespeeld door spelers buiten de coalitie. Men noemt een oplossing sterk consistent als de oplossing van een gereduceerd spel steeds gelijk is aan de projectie van de oplossing van het originele spel. Het is bekend dat 
er geen enkele sterk consistente, niet-lege verfijning van het Nash evenwicht bestaat behalve het Nash evenwicht zelf. We laten echter zien dat het perfecte evenwicht, het propere evenwicht, het zwak perfecte evenwicht en het sequentiële evenwicht (allen verfijningen van het Nash evenwicht) benaderd kunnen worden door een rij sterk consistente oplossingen. We noemen zulke oplossingen limiet consistent.

In Hoofdstuk 9, tenslotte, bekijken we spelen in normale vorm waarbij spelers hun acties kunnen coördineren. Voor deze coördinatie moeten echter kosten gemaakt worden. Binnen dit model introduceren we twee oplossingsconcepten, namelijk de negative excess solution en minimal excess solutions. De eerste oplossing selecteert de strategie-profielen waarbij geen enkele coalitie een reden heeft om af te wijken. Een nadeel van deze oplossing is echter dat een negative excess evenwicht niet altijd hoeft te bestaan. De tweede oplossing, daarentegen, is altijd niet-leeg en kiest de strategie-profielen waarbij de reden van coalities om af te wijken, minimaal is. 



\section{Curriculum Vitae}

Andres Perea y Monsuwé werd op 20 juli 1970 geboren te Palma de Mallorca (Spanje). In 1972 verhuisde hij naar Nederland. Van 1982 tot 1988 volgde hij onderwijs aan het Sintermeerten College in Heerlen waar hij zijn Atheneum B diploma behaalde. Vervolgens maakte hij de overstap naar Duitsland waar hij begon met zijn studie wiskunde aan de Rheinisch-Westfälisch Technische Hochschule Aachen. Tijdens zijn studie was hij twee jaar werkzaam als student-assistent bij de Lehrstuhl C. Nadat hij in 1993 afstudeerde, kreeg hij per 1 juli van hetzelfde jaar een aanstelling als Assistent in Opleiding bij de vakgroep Kwantitatieve Economie aan de Universiteit Masstricht. 